\title{
Experimental and Theoretical Investigation of Instability within a Heated Meniscus
}

\author{
by \\ Michel Garcia \\ M.A.Sc., B.Eng., Carleton University
}

A thesis submitted to the Faculty of Graduate and Postdoctoral Affairs in partial fulfillment of the requirements for the degree of

Doctor of Aerospace Engineering

\author{
Ottawa-Carleton Institute for \\ Mechanical and Aerospace Engineering \\ Department of Mechanical and Aerospace Engineering \\ Carleton University \\ Ottawa, Ontario, Canada \\ 2013
}

Copyright (C)

2013 - Michel Garcia 
Library and Archives

Canada

Published Heritage

Branch

395 Wellington Street

Ottawa ON K1A ON4

Canada
Bibliothèque et

Archives Canada

Direction du

Patrimoine de l'édition

395 , rue Wellington

Ottawa ON K1A ON4

Canada
Your file Votre référence

ISBN: 978-0-494-94533-9

Our file Notre référence

ISBN: $978-0-494-94533-9$
NOTICE:

The author has granted a nonexclusive license allowing Library and Archives Canada to reproduce, publish, archive, preserve, conserve, communicate to the public by telecommunication or on the Internet, loan, distrbute and sell theses worldwide, for commercial or noncommercial purposes, in microform, paper, electronic and/or any other formats.

The author retains copyright ownership and moral rights in this thesis. Neither the thesis nor substantial extracts from it may be printed or otherwise reproduced without the author's permission.
AVIS:

L'auteur a accordé une licence non exclusive permettant à la Bibliothèque et Archives Canada de reproduire, publier, archiver, sauvegarder, conserver, transmettre au public par télécommunication ou par l'Internet, prêter, distribuer et vendre des thèses partout dans le monde, à des fins commerciales ou autres, sur support microforme, papier, électronique et/ou autres formats.

L'auteur conserve la propriété du droit d'auteur et des droits moraux qui protege cette thèse. $\mathrm{Ni}$ la thèse ni des extraits substantiels de celle-ci ne doivent être imprimés ou autrement reproduits sans son autorisation.
In compliance with the Canadian Privacy Act some supporting forms may have been removed from this thesis.

While these forms may be included in the document page count, their removal does not represent any loss of content from the thesis.
Conformément à la loi canadienne sur la protection de la vie privée, quelques formulaires secondaires ont été enlevés de cette thèse.

Bien que ces formulaires aient inclus dans la pagination, il n'y aura aucun contenu manquant. 


\section{Abstract}

The behaviour of heated, evaporating menisci in capillary tubes was experimentally observed and theoretically predicted. Testing was performed with $\mathrm{n}$-pentane as a working fluid evaporating in both ambient air (multicomponent, multiphase environment) and a chamber filled with its saturated vapour (single-component, multiphase environment). Capillary tubes with both thin and thick walls with comparable internal diameters were tested. Thermocouples were embedded inside the thick tubes and epoxied onto the thin tubes. A carbon composite resistive element was deposited onto each tube for electrical heating. A meniscus was positioned between tube thermocouples and electrical heating was provided until the meniscus was observed to oscillate in the axial direction, at which time it was considered destabilised. The experimental temperature gradient at the onset of instability was found to be significantly different for thin and thick tubes of comparable inner diameters. Thin tube results were determined to be more representative of the axial temperature gradient at the tube inner wall. Oscillations were observed and recorded using a microscopecamera assembly. Meniscal instability occurred at a higher temperature gradient for testing while exposed to ambient air than for testing in a pure n-pentane environment. A meniscus inside a smaller inner diameter tube was found to be capable of sustaining a larger temperature gradient than that for a meniscus inside a larger inner diameter tube.

A leading-order scaled equation describing the evolution of the thin film was derived and a perturbation analysis was applied to obtain a stability criterion. A characteristic 
height was chosen as the height at which the evaporative mass flux was a maximum. The meniscus half channel width was chosen as the characteristic length normal to the characteristic height. A numerical thin film model was created to obtain the characteristic height from the thin film solution profile together with the calculated value of the stability criterion. Comparison with experimental results showed that the model has some predictive ability in determining the onset of evaporating meniscal instability in a heated capillary tube. Results of this study can be used to design and characterise the operational limits of phase change heat transfer devices using evaporation from a meniscus as would occur in heat pipes. 
"ignorance more frequently begets confidence than does knowledge"

-Charles Darwin 


\section{Acknowledgments}

I would first and foremost like to thank my supervisor, Professor Tarik Kaya for his guidance and patience during my time as a graduate student. I have enjoyed our discussions about theory that helped me appreciate the difference between what we know and what we think we know. The insightful advice on experimental work provided by Professor John Gaydos was quite useful from my time spent as an undergraduate summer student to the present. I would also like to thank Professor Steve McGarry for helping me with the deposition process in heater manufacturing.

Help from the technical staff at Carleton is also appreciated. Spending years in the ivory tower, it was invaluable to get grounding practical engineering advice. Discussions with Steve Truttmann and Stephan Biljan helped develop the growth of my 'engineering judgement'.

I would like to thank my high school friend Thom Cholowski who delved with me into the hobby of experimental rocketry. I consider our application of basic physics and construction methods to design and build working flight vehicles as seminal in the pursuit of engineering as a profession when faced with the proverbial fork in the road.

Finally, I would like to thank my family who have helped me since before I can remember and throughout the duration of my schooling. I imagine I would have not gone this far in school without your support. 


\section{Table of Contents}

Abstract ii

Acknowledgments $\quad$ v

Table of Contents vi vi

List of Tables $\quad$ xii

List of Figures $\quad$ xiii

$\begin{array}{ll}\text { Nomenclature } & \text { xviii }\end{array}$

1 Introduction 1

1.1 Motivation for study $\ldots \ldots \ldots \ldots \ldots$

1.2 Overview of applications $\ldots \ldots \ldots \ldots \ldots$

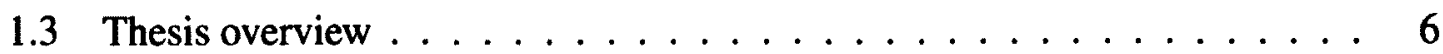

2 Conceptual background and literature review 8

2.1 Interfacial forces $\ldots \ldots \ldots \ldots$

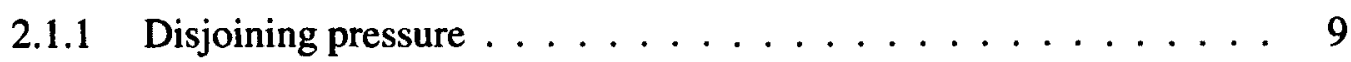

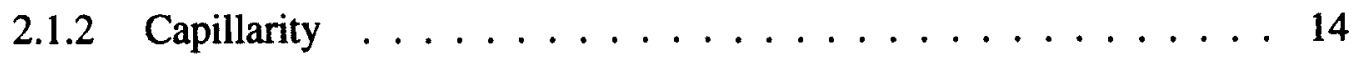

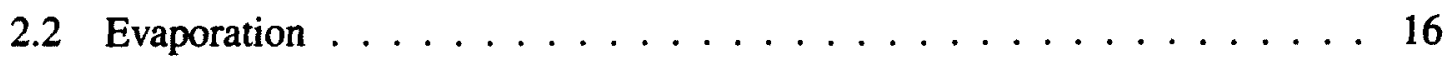




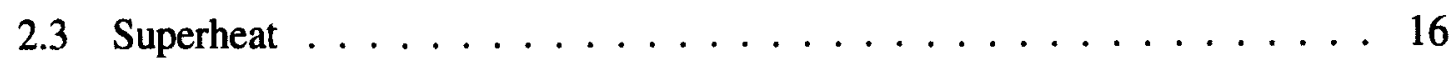

2.4 Interfacial thermal resistance to evaporation $\ldots \ldots \ldots \ldots \ldots$

2.5 Stability theory . . . . . . . . . . . . . . . . . 19

2.5.1 Mechanisms leading to instability . . . . . . . . . . 19

2.6 Relevant previous research $\ldots \ldots \ldots \ldots \ldots \ldots \ldots \ldots$

2.6 .1 Conclusions . . . . . . . . . . . . . . . . . . . . 39

3 Experimental setup $\quad \mathbf{4 0}$

3.1 Overview . . . . . . . . . . . . . . 40

4 Experimental procedure and results 44

4.1 Experimental procedure . . . . . . . . . . . . . . . 44

4.1.1 Positioning the tube $\ldots \ldots \ldots \ldots \ldots \ldots$

4.1.2 Sealing the chamber $\ldots \ldots \ldots \ldots \ldots \ldots$

4.1.3 Evacuating the chamber $\ldots \ldots \ldots \ldots \ldots$

4.1 .4 Filling the reservoir . . . . . . . . . . . . 46

4.1 .5 Data recording . . . . . . . . . . . . 47

4.2 Data analysis . . . . . . . . . . . . . . . . 49

4.2 .1 Heater power . . . . . . . . . . . . . . . . . . 49

4.2.2 Steady state determination $\ldots \ldots \ldots \ldots \ldots \ldots$

4.2.3 Meniscus imaging $\ldots \ldots \ldots \ldots \ldots \ldots \ldots \ldots$

4.2.4 Thermocouple spacing . . . . . . . . . . . . . . . 54

4.2.5 Temperature and power versus time $\ldots \ldots \ldots \ldots \ldots \ldots$

4.2.6 Comparing the measured temperature gradient for thick and thin

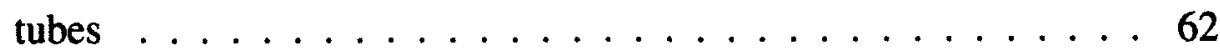

4.2.7 Average temperature difference variation with applied power . . . 63

4.2 .8 Uncertainty analysis $\ldots \ldots \ldots \ldots \ldots 4 \ldots \ldots$ 
5 Stability theory $\quad 75$

5.1 Geometry and coordinate system . . . . . . . . . . . 75

5.2 Governing equations and boundary conditions . . . . . . . . . 77

5.2 .1 Mass conservation ...................... 78

5.2 .2 Momentum conservation . . . . . . . . . . . . 78

5.2 .3 Energy conservation ................... 79

5.2.4 Boundary conditions . . . . . . . . . . . . . 79

5.2 .5 Energy jump ..................... 85

5.2.6 Constitutive equation for resistance to evaporation . . . . . . 86

5.3 Scaling the governing equations and boundary conditions . . . . . . . 86

5.3 .1 Scaling parameters .................. 86

5.3 .2 Mass conservation equation scaling . . . . . . . . . . . 90

5.3.3 Momentum conservation equation scaling . . . . . . . . . . . 90

5.3.4 Energy conservation equation scaling ............. 91

5.3.5 Dirichlet boundary conditions scaling ............ 91

5.3.6 Mass flux jump condition scaling ............... 92

5.3.7 Normal stress jump condition scaling . . . . . . . . . . . . 92

5.3.8 Tangential stress jump balance scaling . . . . . . . . . . 93

5.3 .9 Energy jump condition scaling . . . . . . . . . . . . . . 94

5.3.10 Constitutive equation scaling . . . . . . . . . . . . . . 94

5.4 Leading-order approximation . . . . . . . . . . . . . . . . . 94

5.4.1 Power series expressions of variables ............ 95

5.4 .2 Leading-order governing equations ............. 96

5.4.3 Leading-order boundary conditions ... . . . . . . . . . 96

5.5 Solving the governing equations $\ldots \ldots \ldots 9$

5.5.1 Energy jump solution . . . . . . . . . . . . . . 99 
5.5.2 Constitutive equation solution $\ldots \ldots \ldots \ldots$. . . . 99

5.5.3 Normal momentum jump solution $\ldots \ldots \ldots \ldots$

5.5.4 Tangential momentum jump solution $\ldots \ldots \ldots \ldots$

5.5.5 Film height evolution equation obtained using the interfacial kinematic condition . . . . . . . . . . . . . . 102

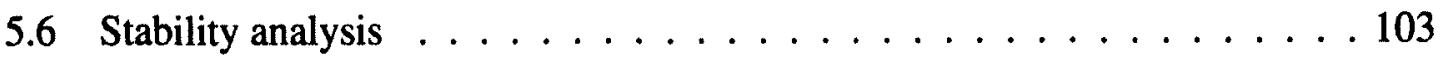

6 Comparison between theory and experiment 112

6.1 Mathematical modelling of an evaporating meniscus $\ldots \ldots \ldots \ldots 112$

6.1.1 Interfacial forces $\ldots \ldots \ldots \ldots \ldots \ldots \ldots$

6.1 .2 Flow within the thin film . . . . . . . . . . . 114

6.2 Thin film profile on the verge of instability . . . . . . . . . 119

6.2.1 Relation of characteristic lengths to stability criterion . . . . . 119

6.2.2 Matching the thin film and stability criterion superheats . . . . 120

6.3 Quantitative comparison of theory with experiment $\ldots \ldots \ldots \ldots 122$

6.3.1 Significance of temperature gradient comparison for stability prediction . . . . . . . . . . . . . . . 124

6.3.2 Quantifying the experimental and theoretical temperature gradients 126

7 Conclusions and recommendations for future work 129

7.1 Conclusions . . . . . . . . . . . . . . . . . . . . . . . . . 129

7.1.1 Experimental conclusions $\ldots \ldots \ldots \ldots \ldots \ldots$

7.1.2 Theoretical conclusions $\ldots \ldots \ldots \ldots \ldots \ldots$

7.2 Recommendations. . . . . . . . . . . . . . . . . 132

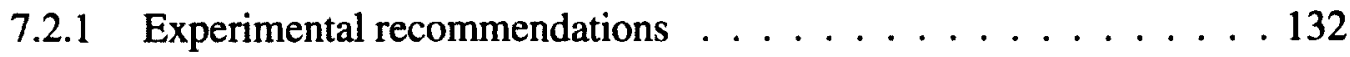

7.2.2 Continuous measurement, adjustment, and recording of the reservoir position $\ldots \ldots \ldots \ldots \ldots \ldots \ldots \ldots \ldots \ldots \ldots$ 
7.2.3 Leak reduction in the vacuum chamber . . . . . . . . . . 134

7.2.4 Priming of liquid fill line to avoid vapour pulsing into chamber . . 134

7.2 .5 Chamber rewiring $\ldots \ldots \ldots \ldots \ldots \ldots \ldots \ldots$

7.2.6 Thermocouple placement jig . . . . . . . . . . . . 135

7.2.7 Measuring wall heat transfer to meniscus $\ldots \ldots \ldots \ldots$

7.2 .8 Heater position variation $\ldots \ldots \ldots \ldots \ldots \ldots$

7.2.9 Constant wall temperature testing $\ldots \ldots \ldots \ldots \ldots \ldots$

7.2 .10 Reservoir cooling . . . . . . . . . . . . . . . . . 138

7.2.11 Theoretical recommendations $\ldots \ldots \ldots \ldots \ldots \ldots$

7.2.12 Cylindrical coordinate model . . . . . . . . . . . . . 138

7.2.13 Evaporation model development . . . . . . . . . . . . . 139

7.2.14 Unified film and bulk solution . . . . . . . . . . . . 139

References

A Details of experimental setup 148

A.1 Vibration isolation $\ldots \ldots \ldots \ldots \ldots \ldots \ldots \ldots \ldots$

A.2 Stationary subsystems . . . . . . . . . . . . . . . . 149

A.2.1 Testing support base $\ldots \ldots \ldots \ldots \ldots \ldots \ldots$

A.2.2 Testing baseplates $\ldots \ldots \ldots \ldots \ldots \ldots \ldots \ldots$

A.2.3 Ambient test chamber $\ldots \ldots \ldots \ldots \ldots \ldots \ldots \ldots$

A.2.4 Vacuum test chamber . . . . . . . . . . . . 152

A.2.5 Microscope . . . . . . . . . . . . . . . 154

A.2.6 Data acquisition $\ldots \ldots \ldots \ldots \ldots \ldots \ldots \ldots \ldots$

A.2.7 Static feedthroughs . . . . . . . . . . . . . . . 159

A.3 Positioning subsystems $\ldots \ldots \ldots \ldots \ldots \ldots \ldots \ldots \ldots \ldots \ldots$

A.3.1 Vertical motion of the bell jar using a crane $\ldots \ldots \ldots 160$ 
A.3.2 Translation and rotation of chamber $\ldots \ldots \ldots \ldots \ldots$

A.3.3 Reservoir . . . . . . . . . . . . . . . . 163

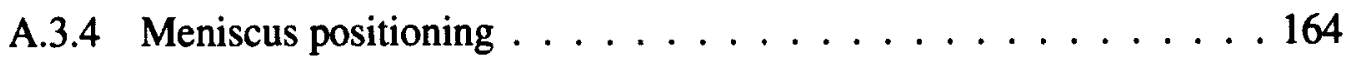

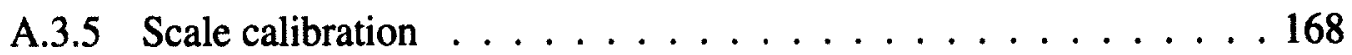

A.3.6 Microscope positioning . . . . . . . . . . . . . . 169

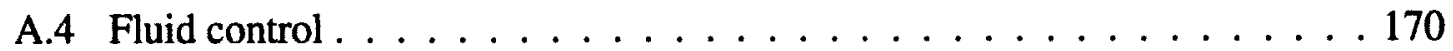

A.4.1 Chamber sealing $\ldots \ldots \ldots \ldots \ldots \ldots \ldots \ldots$

A.4.2 Chamber evacuation $\ldots \ldots \ldots \ldots \ldots \ldots \ldots \ldots$

A.4.3 Liquid manipulation before and after testing . . . . . . . . . 174

A.4.4 Liquid delivery to reservoir for vacuum testing . . . . . . 175

A.4.5 Liquid delivery to reservoir for ambient testing . . . . . . . 179

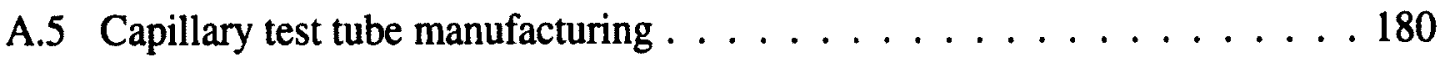

A.5.1 Stock thick and thin tubes $\ldots \ldots \ldots \ldots \ldots \ldots$

A.5.2 Thick tube thermocouples $\ldots \ldots \ldots \ldots \ldots \ldots \ldots$

A.5.3 Thin tube thermocouples . . . . . . . . . . . . . . 184

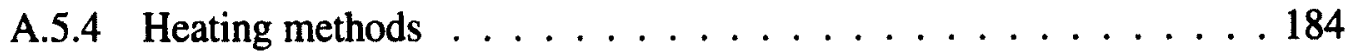

A.5.5 Progression of tube manufacturing . . . . . . . . . . . 189

A.5.6 Lead bracket clamp . . . . . . . . . . . . . . . . . 190

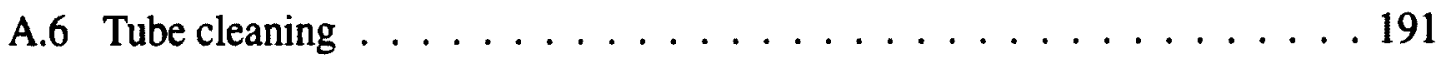

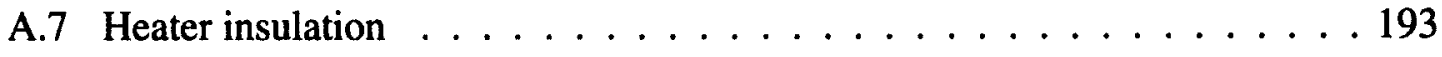

A.8 Reservoir cooling . . . . . . . . . . . . . . . . . . . 195

A.8.1 Possible cooling methods . . . . . . . . . . . . . . . . 195

A.8.2 External cooling . . . . . . . . . . . . . . 196

A.8.3 Internal liquid heat exchanger $\ldots \ldots \ldots$. . . . . . . 196

A.8.4 Thermoelectric cooling . . . . . . . . . . . . . . . . . . 197

A.8.5 Future testing $\ldots \ldots \ldots \ldots \ldots \ldots \ldots \ldots \ldots$ 


\section{List of Tables}

4.1 Data for obtaining magnification scaling factors for $1 \mathrm{~mm}$ ID thin, $1.2 \mathrm{~mm}$ ID thick, and $0.6 \mathrm{~mm}$ ID thick tubes in vacuum testing . . . . . . . . 54

4.2 Axial distance between thermocouples for thick and thick tubes . . . . . . 56

4.3 Meniscus thermocouple temperatures and gradient at onset of instability. *Meniscus still considered stable . . . . . . . . . . . . . . . 62

4.4 Coefficients for DAQ polynomial conversion of voltage to temperature . . . 66

6.1 Comparison of stability criterion with experimental work with reservoir of

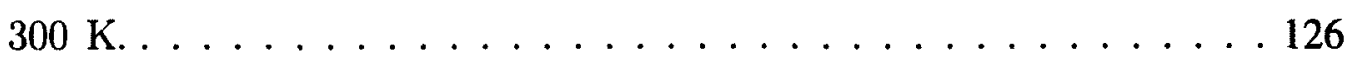

A.1 Analysis summary for upper limit of liquid velocity entering internal reservoir for vacuum and ambient cases . . . . . . . . . . . . . . . 178 


\section{List of Figures}

1.1 Thermosyphon operating with gravity $\ldots \ldots \ldots \ldots \ldots$

1.2 Heat pipe operating against gravity: 1. low pressure liquid evaporates, 2. high pressure vapour travels down pipe, 3 . vapour condenses, 4. high pressure liquid travels up pipe $\ldots \ldots \ldots \ldots \ldots \ldots$

1.3 Thin film formed between heated surface with attached bubble $\ldots \ldots .6$

2.1 Hypothetical apparatus to quantify the disjoining pressure $[12] \ldots \ldots 10$

2.2 Intermolecular interaction of three phases yielding Hamaker constant . . . 12

2.3 Pressure versus volume diagram with isotherm and spinodal curve to explain superheat $[17] \ldots \ldots \ldots \ldots \ldots \ldots$

2.4 Rayleigh-Taylor instability demonstrated by the inability of a gas at higher pressure to support a liquid at lower pressure . . . . . . . . . . . 20

2.5 Kelvin-Helmholtz instability with high pressure at troughs and low pres-

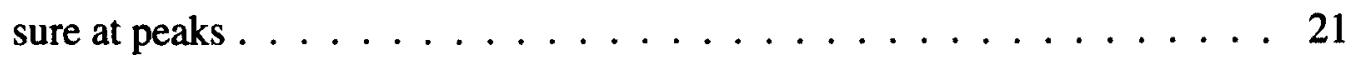

2.6 Vapour recoil mechanism amplifying perturbation by driving liquid in trough toward crest upward . . . . . . . . . . . . . . . 21

2.7 Thermocapillarity mechanism amplifying perturbation by pulling liquid from lower surface tension troughs to higher surface tension crests . . . . . 22

2.8 Thin film rupture $\ldots \ldots \ldots \ldots \ldots \ldots \ldots \ldots \ldots \ldots \ldots \ldots$ 
2.9 Linearly increasing thin film evolution with instability originating in thicker

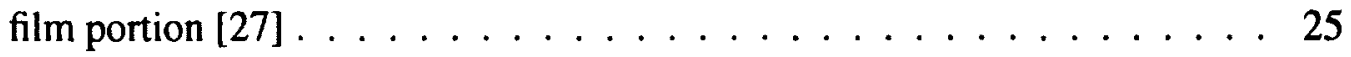

2.10 Thin film as part of meniscus . . . . . . . . . . . . 27

2.11 Meniscus capillary rise $[32] \ldots \ldots \ldots \ldots$

2.12 Heated film test setup with infrared observation camera [36] . . . . . . 30

2.13 Convection (Marangoni) rolling near interface visualised using seeding par-

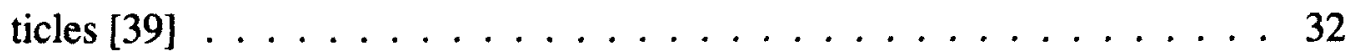

2.14 Convection rotation reversal when reversing temperature difference along

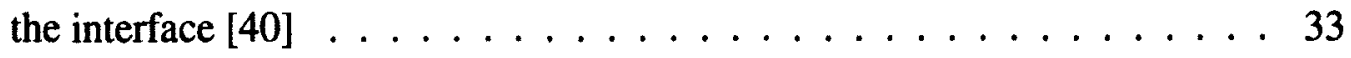

2.15 Capillary height variation for a methanol meniscus with applied power [41] 34

2.16 Average heat transfer coefficient variation with temperature. Mass flux a) 10 , b) 15 , c) 20 , d) $25 \mathrm{gm}^{-2} \mathrm{~s}^{-1}[44] \ldots \ldots 37$

2.17 Experimental setup of a $3 \mathrm{~mm} \times 3 \mathrm{~mm} \times 43 \mathrm{~mm}$ heated cuvette viewed parallel and normal to gravity $[45] \ldots \ldots 37$

3.1 Test setup: 1. test base, 2. chamber, 3. microscope, 4. external reservoir, 5. bell jar crane, 6. chamber positioning control . . . . . . . . . . . 42

4.1 Thin tube temperature change percentage with input power and imposed limits for assumed thermal equilibrium $\pm 0.01 \% \ldots \ldots$. . . . . . . 51

4.2 Thick tube temperature change percentage with input power and imposed limits for assumed thermal equilibrium $\pm 0.01 \% \ldots \ldots$. . . . . . 51

4.3 Stable meniscus as was seen during testing with foreground reticle . . . . . 53

4.4 Meniscus oscillation from baseline shown at $\mathrm{t}=0 \mathrm{~s}$, dropping to a minimum at $\mathrm{t}=1.5 \mathrm{~s}$, and returning near origin at $\mathrm{t}=3.0 \mathrm{~s}$ for $1.0 \mathrm{~mm}$ inner diameter

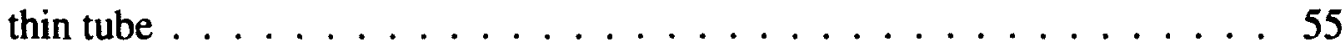


4.5 Temperature for thermocouples above and below meniscus with power input for $1.0 \mathrm{~mm}$ ID thin tube in ambient $\ldots \ldots \ldots$. . . . . . . . . 57

4.6 Temperature for thermocouples above and below meniscus with power input for $1.0 \mathrm{~mm}$ ID thin tube in vacuum $\ldots \ldots . \ldots 59$

4.7 Temperature for thermocouples above and below meniscus with power input for $1.2 \mathrm{~mm}$ inner diameter thick tube in vacuum . . . . . . . . 60

4.8 Temperature for thermocouples above and below meniscus with power input for $0.6 \mathrm{~mm}$ ID thick tube in vacuum ............. . . 60

4.9 Temperature for thermocouples above and below meniscus with power input for $0.5 \mathrm{~mm}$ ID thin tube in vacuum $\ldots \ldots \ldots 1$

4.10 Thermocouple average temperature change versus applied power for thick and thin tubes for vacuum and ambient testing . . . . . . . . . 63

5.1 Geometry of a curved thin film evaporating on heated surface, Top: base state and geometry for unit vectors. Bottom: perturbed state $\ldots \ldots$. . . 76

5.2 Viscous stress acting on a two-dimensional fluid element . . . . . . 83

6.1 Extended meniscus containing the thin film $\ldots \ldots \ldots 113$

6.2 Thin film profile solution algorithm . . . . . . . . . . . . 120

6.3 Iterative superheat search to match thin film superheat to stability criterion . 121

6.4 Thin film profile, $R_{\infty}=0.5 \mathrm{~mm}$ with associated evaporative mass flux: $J$, characteristic length: $x_{c}=0.5 \mathrm{~mm}$, and $\Delta T_{\mathrm{SH}} / x_{c}=13.0 \mathrm{~K} / \mathrm{mm} \ldots 122$

7.1 Parallel plate testing with heater centred above interface . . . . . . . 133

7.2 Vent for vapour to escape away from reservoir . . . . . . . . . . . 135

7.3 Quasi-constant wall temperature near meniscus when using two heaters . . 137

A.1 The $3 \mathrm{~mm}$ diameter glass beads in a reservoir to dampen vibrations . . . . 149 


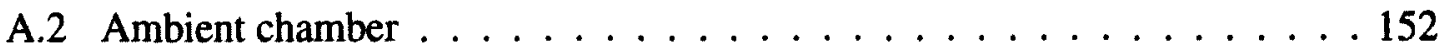

A.3 Vacuum chamber $\ldots \ldots \ldots \ldots \ldots \ldots \ldots \ldots \ldots \ldots$

A.4 External vacuum chamber components . . . . . . . . . . . 153

A.5 LED spotlight for chamber illumination through back window opposite of microscope . . . . . . . . . . . . . . . 155

A.6 Screen display reticle $\ldots \ldots \ldots \ldots \ldots \ldots \ldots \ldots \ldots$

A.7 Power and thermocouple plugs inside of chamber for connection before closing the chamber and disconnection after opening the chamber $\ldots \ldots 160$

A.8 Bell jar moving crane $\ldots \ldots \ldots \ldots \ldots \ldots$

A.9 Safety spacers for connecting and disconnecting feedthroughs from chamber baseplate to bell jar . . . . . . . . . . . . . 161

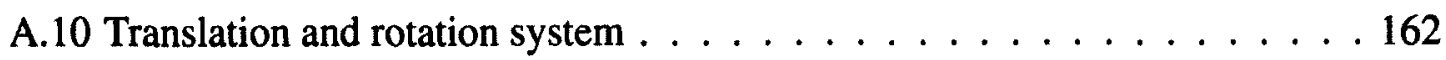

A.11 Meniscus height drop with voltage for ambient testing of $1 \mathrm{~mm}$ ID thin tube where the points at the same voltage represent different times for that given voltage. After voltage is applied and meniscus falls; (square) and before raising voltage to the next step; (triangle) for that given voltage setting. The total drop at the test end after cooling to ambient; (circle). . . . . . 165

A.12 Reservoir position control and measurement in ambient: 1. rotary feedthrough, 2. linear stage, 3. deflectometer, 4. microscope $\ldots \ldots$. . . . . . 167

A.13 Rotary mechanism to move reservoir . . . . . . . . . . . . 167

A.14 Screw jack microscope positioning mechanism . . . . . . . . . 169

A.15 Evacuated chamber pressure increase with applied torque after 15 minutes .171

A.16 Vacuum plumbing system . . . . . . . . . . . . . . . . 172

A.17 Measured chamber pressure over extended time with needle valve opening . 173

A.18 Liquid plumbing system $\ldots \ldots \ldots \ldots \ldots \ldots$ 
A.19 Parameters for obtaining an upper limit for the outlet velocity inside the vacuum chamber, $v_{2} \ldots \ldots \ldots \ldots \ldots \ldots \ldots \ldots \ldots$

A.20 Reservoir under ambient testing $\ldots \ldots \ldots$. . . . . . . . 179

A.21 Drilling $1 \mathrm{~mm}$ diameter holes in glass tubes $\ldots \ldots \ldots \ldots \ldots$

A.22 Helix heater configuration: 1. nichrome heating element, 2. thick walled capillary tube, 3 . soldered junction, 4 . insulated wire, 5. tensioner clamp, 6. fixed clamp, 7. base to carry tensioning load $\ldots \ldots \ldots 186$

A.23 Heater setup using film wrapped around copper annulus . . . . . . . . . 187

A.24 Rotary drive for heater deposition $\ldots \ldots \ldots \ldots \ldots \ldots$

A.25 Evolution of capillary tube from stock to working: 1. offcut stock, 2 . length cut to size, 3a. heater silver contact band, $3 \mathrm{~b}$. bare carbon composition, $3 \mathrm{c}$. TC holes 3d. taped off opening, 4a. epoxy layer, $4 b$. heater lead, 4c. TCs, 4d. lead bracket clamp, $4 \mathrm{e}$. support member, $4 \mathrm{f}$. crimped leads, $4 \mathrm{~g}$. TC subminiature connectors . . . . . . . . . . . . . . . . . 190

A.26 Thin tube $(0.5 \mathrm{~mm} \mathrm{ID)}$ ) with thermocouples and lead bracket clamp $\ldots . .191$

A.27 Tube being washed in the ultrasonic cleaner double bath of acetone in water 193

A.28 TEC setup for temperature control: 1. funnel, 2. reservoir cover, 3. cold plate (above TEC), 4. heat sink (below TEC) ․ . . . . . . . . . 198

A.29 Ambient test showing partial effectiveness of TEC with $20 \mathrm{~W}$ input on TEC 199

A.30 Attempting to cool chamber reservoir: 1. evaporative cooling from $n-$ pentane entering vacuum chamber, 2 . liquid n-pentane contacts reservoir thermocouple, 3. TEC on and unable to cool reservoir . . . . . . . . 201 


\section{Nomenclature}

A area $\mathrm{m}^{2}$

$\bar{A} \quad$ dispersion constant $\quad \mathrm{J}$

$A_{H} \quad$ Hamaker constant J

$B \quad$ bias uncertainty

Bo Bond number $\left(\frac{\rho g L_{c}^{2}}{\sigma}\right)$

$\begin{array}{lll}c_{p} & \text { specific heat at constant pressure } & \mathrm{J} \mathrm{kg}^{-1} \mathrm{~K}^{-1}\end{array}$

E evaporation number $\left(\frac{k \Delta T}{\mu \mathcal{L}}\right)$

F force N N N

f function

$f_{e} \quad$ main electronic absorption frequency $\quad \mathrm{Hz}$ 
$f_{x_{i}} \quad$ sensitivity parameter

g gravitational acceleration

$\mathrm{ms}^{-2}$

$G r_{L} \quad$ Grashof number $\left(\frac{g \beta \Delta T L^{3}}{\nu^{2}}\right)$

$H \quad$ scaled perturbed film height

h liquid film height, convective heat transfer coefficient

$\mathrm{m}, \mathrm{Wm}^{-2} \mathrm{~K}^{-1}$

6 capillary height

m

$h_{c} \quad$ vertical characteristic length

m

$h_{P} \quad$ Planck constant $\left(6.626 \times 10^{-34}\right)$

$\mathrm{m}^{2} \mathrm{~kg} \mathrm{~s}^{-1}$

I current

A

$i \quad$ imaginary unit

J mass flux

$\mathrm{kg} \mathrm{s}^{-1} \mathrm{~m}^{-2}$

K interfacial thermal resistance

$\mathrm{Ks} \mathrm{m}^{2} \mathrm{~kg}^{-1}$

k thermal conductivity

$\mathbf{W} \mathbf{m}^{-1} \mathbf{K}^{-1}$

$k_{B} \quad$ Boltzmann constant $\left(1.380 \times 10^{-23}\right)$

$\mathrm{kg} \mathrm{m}^{2} \mathrm{~s}^{-2} \mathrm{~K}^{-1}$

L distance between thermocouples

m 
$\mathcal{L} \quad$ latent heat

1 length

M molecular mass

$\dot{m} \quad$ mass flow rate

Ma Marangoni number $\left(\frac{c_{p} \gamma \Delta T h_{c}^{2}}{x_{c} \nu k}\right)$

n refractive index

$\mathrm{Nu} \quad$ Nusselt number $\left(\frac{h L}{k_{v}}\right)$

P pressure

$\mathrm{Pa}$

$\mathcal{P} \quad$ power

W

Pr Prandtl number $\left(\frac{\mu c_{p}}{k}\right)$

Q heat transfer

W

R specific gas constant, resistance

$\mathrm{J} \mathrm{kg}^{-1} \mathrm{~K}^{-1}, \Omega$

$R_{1,2}$ interfacial radii of curvature

$\mathrm{m}^{-1}$

$R_{\theta} \quad$ absolute thermal resistance

$\mathrm{K} \mathrm{W}^{-1}$

$R_{u} \quad$ universal gas constant (8.314)

$\mathrm{J} \mathrm{K}^{-1} \mathrm{~mol}^{-1}$ 


\section{Ra Rayleigh number $(P r \times G r)$}

$S_{u} \quad$ precision uncertainty

T temperature

${ }^{\circ} \mathrm{C}, \mathrm{K}$

$\begin{array}{lll}\mathcal{T} & \text { stress tensor } & \mathrm{Pa}\end{array}$

$t$ time

$T_{f} \quad$ film temperature

${ }^{\circ} \mathrm{C}, \mathrm{K}$

$\Delta T_{\exp }$ measured thermocouple temperature difference

${ }^{\circ} \mathrm{C}$

$\Delta T_{\mathrm{SH}}$ theoretical superheat

${ }^{\circ} \mathrm{C}$

$\mathcal{U}_{95}$ total uncertainty with $95 \%$ confidence

$u \quad$ liquid velocity scalar in direction parallel to solid surface

$\mathrm{m} \mathrm{s}^{-1}$

$\mathcal{V} \quad$ volume

$\mathrm{m}^{3}$

$v \quad$ liquid velocity scalar in direction normal to solid surface

$\mathrm{m} \mathrm{s}^{-1}$

$\vec{V} \quad$ liquid velocity vector

$\mathrm{m} \mathrm{s}^{-1}$

$V_{l} \quad$ molar volume

$\mathrm{m}^{3} \mathrm{~mol}^{-1}$

$X \quad$ general direction 
$\mathcal{X}$ mole fraction

$\tilde{x} \quad$ ratio of vertical to horizontal characteristic lengths $\left(\frac{h_{c}}{x_{c}}\right)$

$x_{c} \quad$ horizontal characteristic length

m

\section{Greek}

$\alpha \quad$ accommodation coefficient

$\beta \quad$ volumetric thermal expansion coefficient

$\mathrm{K}^{-1}$

$-\gamma \quad$ surface tension change with temperature

$\mathbf{N} \mathbf{m}^{-1} \mathbf{K}^{-1}$

$\kappa \quad$ curvature

$m^{-1}$

$\lambda \quad$ disturbance wave number

$\mu \quad$ dynamic viscosity

$\mathrm{kg} \mathrm{m}^{-1} \mathrm{~s}^{-1}$

$\nu \quad$ kinematic viscosity

$\mathrm{m}^{2} \mathrm{~s}^{-1}$

$\Pi$ disjoining pressure

$\mathrm{Pa}$

$\rho$ density

$\mathrm{kg} \mathrm{m}^{-3}$

$\sigma \quad$ surface tension

$\mathrm{N} \mathrm{m^{-1 }}$

$\sigma_{\text {st }} \quad$ standard deviation 
$\theta \quad$ dimensionless temperature $\left(\frac{T-T_{v}}{T_{w}-T_{v}}\right)$

$\theta_{a}$ angle $\quad$ rad

$\varepsilon \quad$ relative dielectric permittivity

$\varphi \quad$ measure of averaged local temperature fluctuation

$\vartheta \quad$ specific volume

$\mathrm{m}^{3} \mathrm{~kg}^{-1}$

\section{Subscripts}

a absolute

act actual

al alignment

bot bottom thermocouple

c capillary, characteristic

$\infty \quad$ at intrinsic meniscus

$1 \quad$ liquid

lv liquid vapour interface

ret reticle spacing 
sur surface

top top thermocouple

t time

u uncertainty

v vapour

w wall

x direction parallel to solid surface

y direction normal to solid surface

\section{Superscripts and overscripts}

- mean value

$i \quad$ index for direction parallel to wall

$j \quad$ index for direction normal to wall

' perturbed value

$\sim$ dimensionless

$\star \quad$ pure 


\section{Acronyms}

AWG American wire gage

BSL best straight line

CCD charge-coupled device

CF conflat

DAQ data acquisition system

DC direct current

DGME diethylene glycol monomethyl ether

DN diameter nominal

HOT higher-order term(s)

ID inner diameter

IR infrared

ISO International standards organisation

ITS International temperature scale

LED light emitting diode 
MF magnification factor

OD outer diameter

PIV particle image velocimetry

PVC polyvinyl chloride

QF quick flange

RPM revolutions per minute

RSS root sum squared

SH superheat

SPST single pole single throw

SR screen reticle

TEC thermoelectric cooler

TLC thermochromic liquid crystal 


\section{Chapter 1}

\section{Introduction}

Liquid to vapour phase transition is a phenomenon vital to our existence, yet our understanding of the mechanisms associated with this process is incomplete. Our livelihood has depended on evaporation since the dawn of humanity with the earth's water cycle and it continues today with increased reliance as we cool the devices which help actualise our technological society.

The main objective of this research study is to experimentally observe and theoretically predict the destabilisation of a heated meniscus in a capillary tube in order to obtain a better understanding of the process at the microscopic level and to provide guidance for the design and operation of phase change heat transfer devices.

\subsection{Motivation for study}

Transferring heat for temperature regulation of power plants, machinery, and electronics has gained societal importance as we increase the energy density of our devices. Design requirements tending toward lower power consumption, mass, and volume favours heat to be removed without an active cooling method such as a pump driven flow heat exchanger. 
An attractive alternative is to passively (without power consumption) remove heat. This can be accomplished by using the energy of a heat load to create a pressure difference to move a fluid between the sink and source at different temperatures. To avoid large temperature changes or mass flow rates that would be required to transfer heat sensibly, we can instead utilise a fluid's latent heat of vaporisation. A challenge to using vaporisation for heat transfer is being able to predict and control how the fluid evaporates in order to create and maintain a reliable heat transfer device. Increasing the heat load on the liquid of an operational phase change heat transfer device will increase its fluid temperature up to its saturation temperature for a given pressure. If the heat load is too high, then the liquid phase will boil off and the device will fail to operate. Adding less heat such that boiling does not occur, the heat is transferred via evaporation. For sustained evaporation to occur, liquid must be replenished to replace the evaporated fluid. The replacement fluid travels from a colder region to the location where it will evaporate. This travel is not without concern, as it could potentially disturb the free interface and cause the device to fail via a convection mechanism.

The domain where phase change occurs makes the problem distinct as the length scale is small. Keeping the distance between the heat source and evaporation site small results in a small temperature drop due to conduction. As the length scale decreases, intermolecular interaction between the solid, liquid, and vapour becomes more relevant to the analysis and as such should be explicitly considered to accurately predict the physics.

An aspect that makes predicting the behaviour of an evaporating thin film difficult is the deformable boundary between the liquid and vapour phases. Disturbing the liquidvapour interface results in a capillary wave as the energy from the disturbance interacts with the liquid surface that is in tension. As a result of these waves, phase change heat transfer devices are prone to interfacial oscillation and such motion is known to destabilise the meniscus. It is worthwhile to search for a criterion which predicts the onset of this 
behaviour to prevent device failure when cooling is critically important.

\subsection{Overview of applications}

We will now review some of the numerous applications which can benefit from the research undertaken in this study. Essentially, the development of all processes or devices where evaporation is critical can benefit, but, for the sake of illustration and brevity, more specific applications are mentioned below.

The phase change thermosyphon is a passive heat transfer device which relies on gravity for the passive movement of vapour and liquid. A simple thermosyphon consists of a vertical tube with a fluid such as water evaporating at the base due to the heat source. The vapour then rises to the top via natural convection where it is cooled by the sink and flows back toward the source along the wall. The layout of a simple thermosyphon is shown in Figure (1.1). Beginning with its inception during the Industrial Revolution, the thermosyphon has been used to cool buildings, such as bakeries, and is still used today in such applications where functional orientation is feasible.

A heat pipe is similar in function to a thermosyphon, but it does not require gravitational assistance to operate as shown in Figure (1.2). Instead, fluid motion is accomplished via a wicking material which allows for a capillary pressure difference and flow between phases.

Since the first patent by Gaughler in 1944 [1] and the working prototype by Grover in 1964 [2], the optimisation of heat pipes has been ongoing to match the demand for thermal control of devices ranging from the ubiquitous personal computer, to transcontinental oil pipelines, and to exploratory spacecraft.

The operation of both thermosyphons and heat pipes depends on the integrity of a heated thin film. The main difference is the capillary pressure for a heat pipe is much larger. A small length scale (larger than the thin film length scale) is also required when studying 


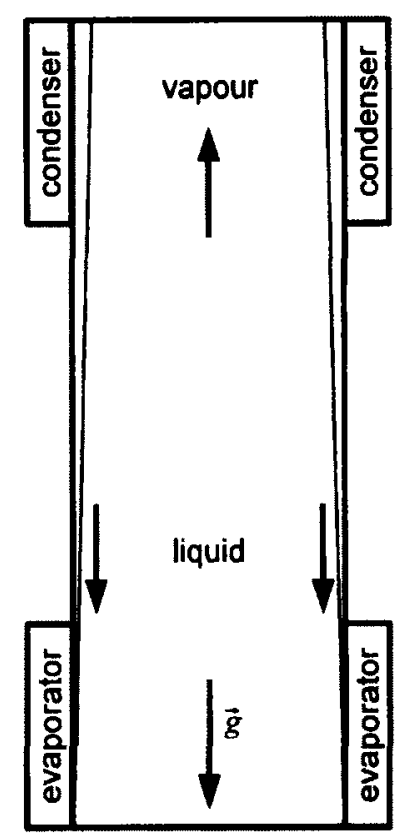

Figure (1.1): Thermosyphon operating with gravity

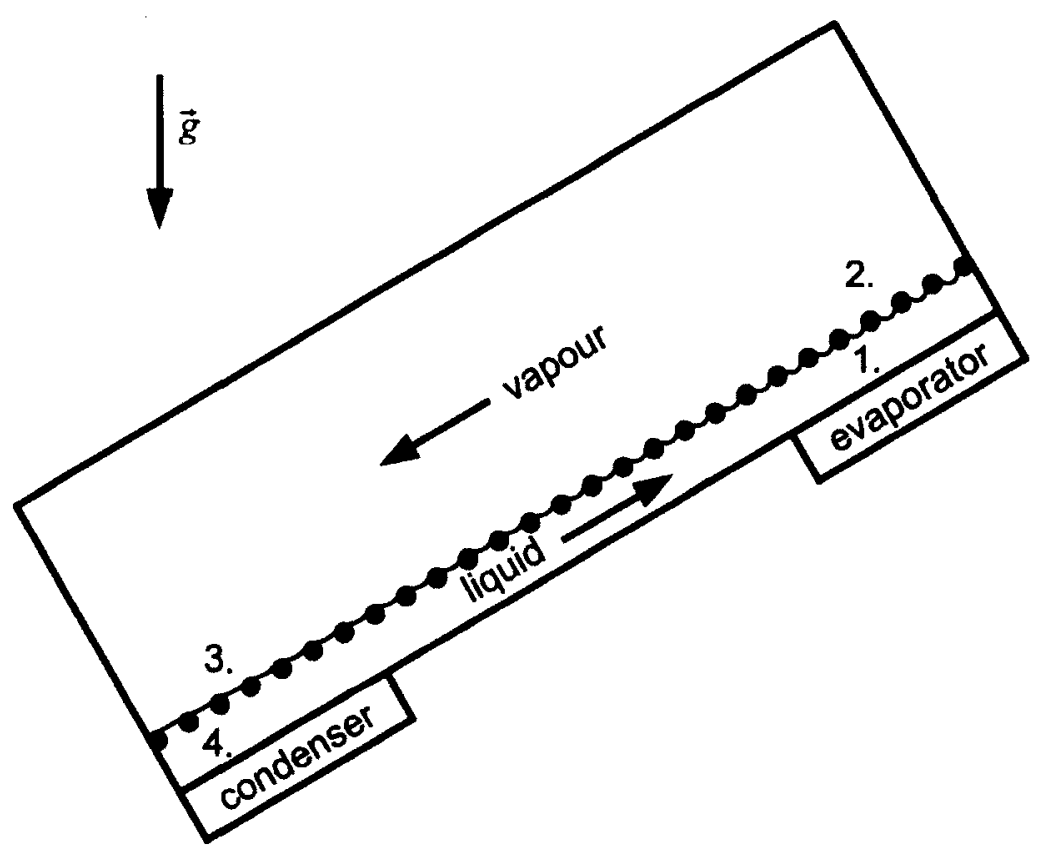

Figure (1.2): Heat pipe operating against gravity: 1. low pressure liquid evaporates, 2 . high pressure vapour travels down pipe, 3. vapour condenses, 4 . high pressure liquid travels up pipe 
a device such as a heat pipe since it employs capillary pressure to sustain a pressure difference between its vapour and liquid phases which it uses for fluid motion. Consideration for inertial and gravitational effects is more relevant for thermosyphon analysis since the liquid film is thicker and driven by gravity.

Using capillary pressure to separate the phases has the benefit of allowing for evaporation to occur more easily than with having an actual membrane as a separator. The interface is also free to self-adjust for the given heat load and fluid charge quantity.

The research performed in this work is related to heat pipes and thermosyphons in that a capillary tube corresponds to a single pore heat pipe. The observed instabilities in the capillary tube give insight as to how a heat pipe will behave. In the case of a thermosyphon, a thin film similar to that later derived in the theoretical model forms about the evaporator and so the model can be extended to quantify its stability by negating the capillary pressure and introducing gravitational effects.

The rods which control nuclear reaction rates are cooled by submersion in a circulating water bath. Bubble formation at a nucleation site on a control rod surface results in a curved thin film between the rod and bubble as sketched in Figure (1.3) noting that in reality the bubble would not be perfectly spherical due to external disturbances. The formation of some bubbles results in more effective heat transfer compared to liquid alone since the bubbles introduce motion for convection as well as absorbing their latent heat of formation. As the heat load is further increased, more bubbles form at the surface and eventually the presence of bubbles will limit the heat that can be transferred at a given temperature since they act as an insulative layer between the rod and liquid. Understanding the behaviour of the thin film between the solid and vapour can help to predict the extent of pool boiling in nuclear cooling and many other critical industrial processes that involve liquid bath cooling. The evaporative development of a microlayer liquid film under a bubble has a significant effect on the growth and detachment of the bubble from its nucleation site [3]. 


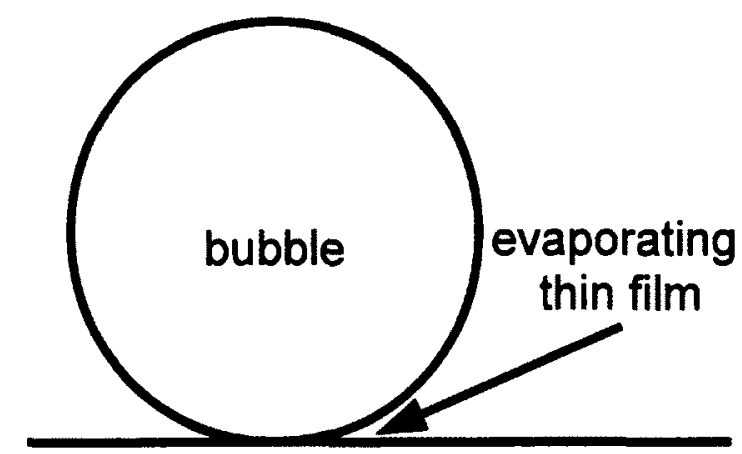

heated surface

Figure (1.3): Thin film formed between heated surface with attached bubble

As a droplet of ink is ejected from a printer nozzle, it lands on a paper substrate and dries. Drying involves the evaporation of the solvent within the droplet which leaves behind the solid ink. By understanding evaporative thin film behaviour, ink formulations which dry predictably and faster can be developed.

Cooling of thrust chambers and nozzles in the realm of rocket propulsion has been achieved via film cooling [4]. Liquid film coolant injection at the wall is used to protect walls by transferring heat via evaporation. Since the injected liquid produces relatively little thrust in comparison to the primary propellant jet, the objective becomes minimisation of the cooling film such that it can still protect the walls sufficiently. High velocity exhaust gases passing over the film disturbs the interface making knowledge of evaporating thin film stability critical to designing the wall cooling system [5].

\subsection{Thesis overview}

In Chapter 2, we will introduce the basic concepts associated with intermolecular forces, evaporation, and destabilising mechanisms to help understand the theoretical and experimental work undertaken in this study. Next, a general review of the existing body of work will be presented as it relates to the current study. Experimental work will be presented 
to illustrate past and contemporary methods used for interfacial heating, visualisation, and temperature measurement. Presented theoretical work will relate to the modelling of an evaporating liquid film and the various known mechanisms that cause instability.

In Chapter 3, we will discuss the experimental setup developed to position, heat, and observe an evaporating meniscus in a capillary tube for both ambient conditions and within a chamber containing $\mathbf{n}$-pentane without air.

Chapter 4 outlines the experimental findings and compares different tube configurations. The axial wall temperature gradient at the onset of instability will be presented for validation of a theoretical model.

Chapter 5 introduces an analytical model to describe an evaporating thin film on a flat substrate at constant temperature. Characteristic lengths were used to scale the equations and to simplify the governing equations to their leading-order terms. An equation was derived to describe the evolution of the thin film height as a free surface subject to initial conditions. A perturbation was applied at the interface and a criterion was established to determine the minimum temperature difference between the solid wall and vapour that will allow the applied perturbation to destabilise the film.

In Chapter 6 we introduce a numerical thin film model to solve for the film profile at the onset of instability. From this model, we iteratively obtain the superheat and characteristic length at the stability limit. We then establish a second criterion which satisfactorily predicts instability when compared with experimental findings.

In Chapter 7 experimental and theoretical conclusions of this study are outlined. In addition, recommendations for future work to complement and supplement this study are given. 


\section{Chapter 2}

\section{Conceptual background and literature}

\section{review}

\subsection{Interfacial forces}

The interfaces where solid, liquid, and vapour meet are considered in this study. We note fluid behaves differently near an interface compared within a bulk region since an interaction exists with the different neighbouring phase. A theory is needed to account for this interaction in order to accurately model the small geometries for comparison with experimental work.

The quantification of surface tension and disjoining pressure which is important for modelling is described below in detail. We note that even though these phenomena are modelled using independent equations, their causality is suspected to depend more strongly upon the same mechanisms at smaller length scales. This is evident when studying the surface tension dependency on the diameter of bubbles [6], [7]. A clear distinction between surface tension and disjoining pressure is that the former and latter act parallel and normal to the interface, respectively [8]. 


\subsubsection{Disjoining pressure}

The affinity or aversion between phases across an interface results in a net attractive or repulsive force, respectively.

Attraction is the result of permanent or fluctuating dipoles caused by differences in electronegativity or electronic motion within a molecule, respectively. Since oscillatory fluctuations tend to a lower energy as neighbouring molecules synchronise their motion, a net force is felt near the interface [9]. These phenomena are termed van der Waals weak body forces [10].

Repulsion is due to the like electrostatic charge between a medium and substrate and is not relevant in this study where we consider the fluid and substrate to have a mutual attraction.

In order to incorporate this body force within a macroscopic model, the effect of the molecular interaction is denoted as an attractive force over the contact area between phases. This term is called the disjoining pressure and is denoted by the symbol $\Pi$ in this study. Disjoining pressure can be defined for a flat film as the pressure difference between the pressure applied at the surface of the film (by the vapour in this study), $P_{\text {sur }}$ and that within the film, $P_{l}[11]$ as

$$
\Pi=P_{\text {sur }}-P_{l}
$$

To understand disjoining pressure from a physical basis, one can consider a pressurised housing placed at a very small distance above a solid substrate in contact with a liquid reservoir shown in Figure (2.1) with the edge effects between the housing and liquid neglected. Taking $P_{l}$ as the pressure on the solid floor and $P_{a}$ as atmospheric pressure, the absolute gas pressure $P_{b}$ inside the housing for the thin film of height $h$ to be in equilibrium 


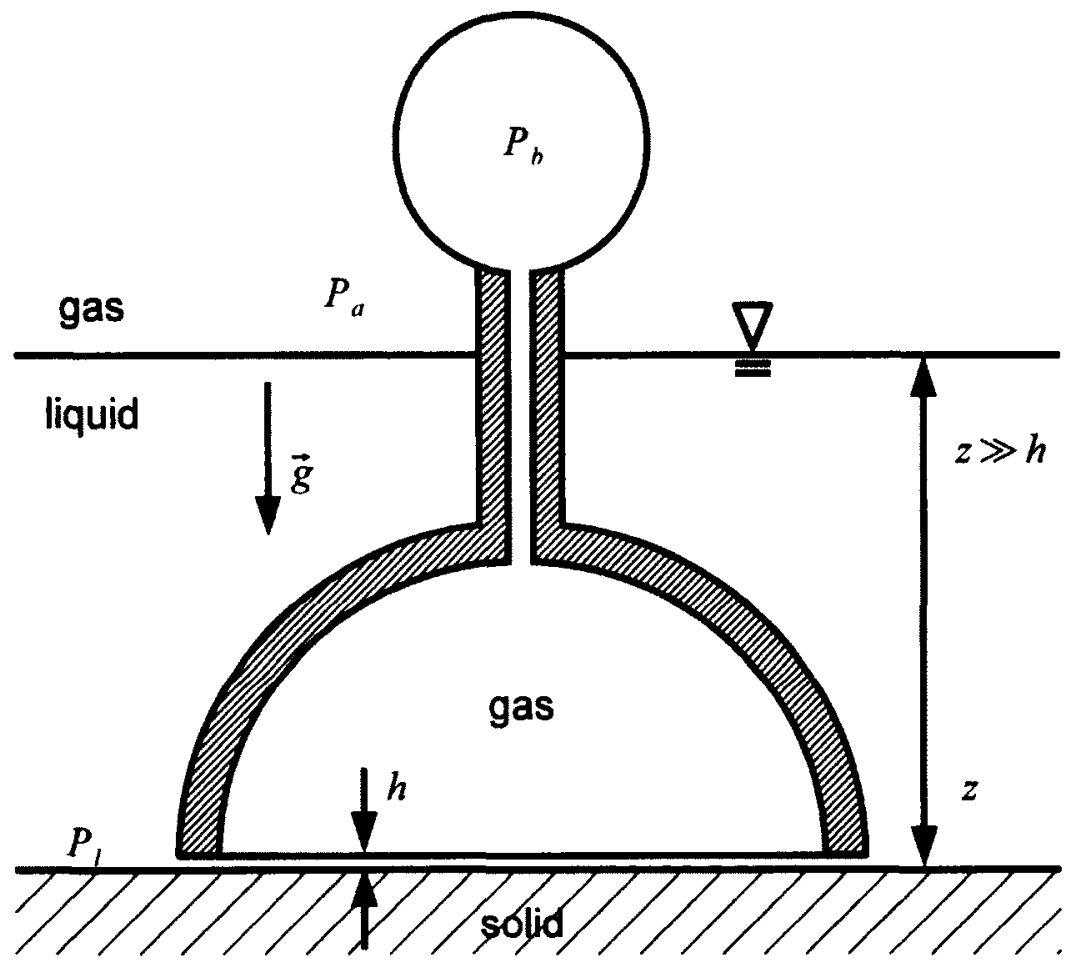

Figure (2.1): Hypothetical apparatus to quantify the disjoining pressure [12]

can be written according to

$$
P_{b}=P_{a}+\rho g(z-h)+\Pi
$$

when the hydrostatic pressure due to the gas is neglected. In the case of the solid and liquid having affinity for one another, the extra pressure or disjoining pressure $\Pi$ is required for mechanical equilibrium and it can be visualised as preventing the suction of fluid from inside the reservoir into the housing. If an aversion instead of an affinity between the liquid and solid existed, $P_{b}$ would be lower to prevent the thinning of the liquid film as it would otherwise tend to leave the housing to distance itself from the solid.

A number of approaches exist to quantify the disjoining pressure and in this study we approximate the disjoining pressure of a curved interface as a flat one in the manner presented in [13] where the disjoining pressure is a function of film thickness [10] according 
to

$$
\Pi=\frac{\bar{A}}{h^{3}}
$$

where $\bar{A}=\frac{A_{H}}{6 \pi}$ is the dispersion constant, $h$ is the film thickness, and $A_{H}$ is the Hamaker constant which is discussed below. Equation (2.3) is used to model the disjoining pressure throughout this work for analytical and computational simplicity. We note that the disjoining pressure of a curved interface is a function of the film profile and can be written using the first and second derivatives of the film thickness [14] to yield Equation 2.4 with $h_{x}$ being the slope of the film with respect to the substrate. Equation 2.4 reduces to Equation (2.3) when the slope is zero.

$$
\Pi=\frac{A_{H}\left(4-3 h_{x}^{2}+3 h h_{x x}\right)}{24 \pi h^{3}}
$$

The absolute value of the disjoining pressure $|\Pi|$ is used in this work to avoid confusion due to inconsistency within the literature. Convention in accordance to [12] relates a negative disjoining pressure to an attractive force between media. Physically, a negative disjoining pressure acts like suction to draw liquid into the thin film from relatively thicker regions adjacent to the thin film. Alternatively, one can view the resultant of disjoining pressure as a pressure change across the liquid-vapour interface tending to reduce the liquid pressure in the same sense as capillary pressure which is described below. Disjoining pressure thus suppresses evaporation as the attraction toward the solid must be overcome in order for a fluid molecule evaporate.

To quantify the disjoining pressure, a model of the thin film was used to obtain a value for the Hamaker constant. The interaction of the liquid film, sandwiched between a solid substrate and vapour is depicted in Figure (2.2) and the corresponding Hamaker constant $A_{H}$ can be approximated within $5 \%$ by solving Equation (2.5) as obtained from Lifshitz theory of van der Waals forces [10] 


\begin{tabular}{ll|ll|} 
macroscopic vapour phase $\varepsilon_{2}=1, n_{2}=1$ & & (2) \\
\hline thin liquid film & $\varepsilon_{3,}, n_{3}$ & constant: $T, f_{e}$ \\
\hline macroscopic solid phase & $\varepsilon_{1,}, n_{1}$ & & (3) \\
\hline
\end{tabular}

Figure (2.2): Intermolecular interaction of three phases yielding Hamaker constant

$$
\begin{aligned}
A_{H} \approx \frac{3}{4} k_{B} T\left(\frac{\varepsilon_{1}-\varepsilon_{3}}{\varepsilon_{1}+\varepsilon_{3}}\right) & \left(\frac{\varepsilon_{2}-\varepsilon_{3}}{\varepsilon_{2}+\varepsilon_{3}}\right) \ldots \\
& +\frac{3 h_{P}}{4 \pi} \int_{f_{1}}^{\infty}\left(\frac{\varepsilon_{1}(i f)-\varepsilon_{3}(i f)}{\varepsilon_{1}(i f)+\varepsilon_{3}(i f)}\right)\left(\frac{\varepsilon_{2}(i f)-\varepsilon_{3}(i f)}{\varepsilon_{2}(i f)+\varepsilon_{3}(i f)}\right) d f
\end{aligned}
$$

where $\varepsilon, f, k_{B}$, and $h_{P}$ are the relative dielectric permittivity, frequency, Boltzmann constant, and Planck constant, respectively. The solution for the Hamaker constant is an approximation of an infinite series as the integral represents leading-order terms. The lower limit of integration, $f_{1}$ is a frequency at the lower end of the infrared region within the light frequency spectrum. The relative dielectric permittivity can be written as a function of frequency $[10]$ as

$$
\varepsilon(i f)=1+\left(n^{2}-1\right) /\left(1+f^{2} / f_{e}^{2}\right)
$$

where $f_{e}$ is the main electronic absorption frequency. By substituting Equation (2.6) into Equation (2.5), the Hamaker constant can be written as a function of known physical parameters via

$$
\begin{aligned}
& A_{H}=A_{f=0}+A_{f>0} \\
& A_{H} \approx \frac{3}{4} k_{B} T\left(\frac{\varepsilon_{1}-\varepsilon_{3}}{\varepsilon_{1}+\varepsilon_{3}}\right)\left(\frac{\varepsilon_{2}-\varepsilon_{3}}{\varepsilon_{2}+\varepsilon_{3}}\right)+\ldots
\end{aligned}
$$




$$
\frac{3 h_{P} f_{e}}{8 \sqrt{2}} \frac{\left(n_{1}^{2}-n_{3}^{2}\right)\left(n_{2}^{2}-n_{3}^{2}\right)}{\left(n_{1}^{2}+n_{3}^{2}\right)^{1 / 2}\left(n_{2}^{2}+n_{3}^{2}\right)^{1 / 2}\left[\left(n_{1}^{2}+n_{3}^{2}\right)^{1 / 2}+\left(n_{2}^{2}+n_{3}^{2}\right)^{1 / 2}\right]}
$$

which assumes the temperature, $T$ and main electronic absorption frequency, $f_{e}$ to be the same in all three phases. The constant temperature assumption is considered valid for this study wherein temperature variation within a particular film is less than $10 \mathrm{~K}$. The vapour phase refractive index, $n_{2}$ and relative dielectric permittivity, $\varepsilon_{2}$ are taken as unity since they are close to the reference vacuum when compared to the much denser solid and liquid phases. Since the dielectric permittivity of vapour is not considered a function of frequency, the choice for $f_{e}$ remains uniquely dependent on phases 1 and 3 shown in Figure (2.2). It is clear that the dielectric permittivities for phases 1 and 3 in the second term of Equation (2.5) decrease as the frequency increases beyond $f_{e}$ from inspection of Equation (2.6). Increasing the frequency results in $\varepsilon_{1} \rightarrow 1$ and $\varepsilon_{3} \rightarrow 1$, therefore the terms in the integrand, $\varepsilon_{1}-\varepsilon_{3} \rightarrow 0$ and $\varepsilon_{2}-\varepsilon_{3} \rightarrow 0$. This implies that non-negligible integral sub-domain will be around the main absorption frequencies of the liquid and solid, therefore the vapour main absorption frequency is not relevant in the analysis. Given the absorption frequencies of liquid n-pentane $\left(3.0 \times 10^{15} \mathrm{~Hz}\right)$ and quartz $\left(3.2 \times 10^{15} \mathrm{~Hz}\right)$ yields a representative main absorption frequency of $f_{e}=3.1 \times 10^{15} \mathrm{~Hz}$ for a thin liquid $\mathrm{n}$-pentane film bound by quartz and vapour on either side.

Alternatively, a composite Hamaker constant can be formulated using the constituent values with respect to vacuum [9] as

$$
A=\left(A_{11}^{1 / 2}-A_{33}^{1 / 2}\right)\left(A_{22}^{1 / 2}-A_{33}^{1 / 2}\right)
$$

where subscripts 1,3 , and 2 correspond to the solid, liquid, and vapour phases respectively. 
Although simpler to calculate, the composite method is not used in this study since it is less accurate than the aforementioned method.

\subsubsection{Capillarity}

The attraction or repulsion between phases in contact results in a curved interface. Since surface tension pulls parallel to an interface, a pressure difference across the interface is required to achieve mechanical equilibrium. The pressure difference across the liquid vapour interface also known as the capillary pressure, $P_{c}$, can be related to its geometry for a static interface via the Young-Laplace equation [8] as

$$
\begin{aligned}
P_{c} & =P_{v}-P_{l} \\
& =\sigma\left(\frac{1}{R_{1}}+\frac{1}{R_{2}}\right)
\end{aligned}
$$

where $\sigma$ is the surface tension, $R_{1}$ and $R_{2}$ are mutually perpendicular radii of curvature. For the theoretical work in this study, we consider two parallel plates of infinite area, with a liquid film in between. Film symmetry is imposed about a plane parallel and equidistant to both plates. Letting $R_{1}$ reside in a plane normal to the plates results in $R_{2} \rightarrow \infty$ since we assume the film does not vary in the direction normal to the plane where $R_{1}$ resides.

We can describe the radius of curvature in Cartesian coordinates as a function of thin

film geometry noting the curvature, $\kappa=R_{1}^{-1}$, can be described mathematically as a function of the film thickness first and second derivatives with respect to $x$ as

$$
\kappa=\frac{h_{x x}}{\left(1+h_{x}^{2}\right)^{\frac{3}{2}}}
$$


with $h$ being the film thickness and the subscript $x$ denoting the derivative in the direction parallel to the plates. Combining Equations (2.9b) and (2.10), we can write the capillary pressure, $P_{c}$ as

$$
P_{c}=\frac{\sigma h_{x x}}{\left(1+h_{x}^{2}\right)^{\frac{3}{2}}}
$$

Variation in surface tension along the interface results in a force pulling in the direction of higher surface tension. For a small temperature change as considered in this study, the surface tension can be modelled as linearly varying with temperature as

$$
\sigma=\sigma_{o}-\gamma\left(T-T_{\text {sat }}\right)
$$

where the reference temperature is taken as the saturation temperature for $\sigma_{o}$ and it is considered equal to the vapour temperature in this study. The symbol $-\gamma$ denotes the surface tension change with temperature and is negative for pure liquids but positive for some mixtures [15]. Surface tension decreases with increasing temperature since intermolecular spacing increases with temperature, thereby lowering attraction to its gaseous phase as both phases increase their similarity. Surface tension for a pure fluid will decrease with increasing temperature until it vanishes at the critical temperature and along the pseudo-critical line [12].

In this study we neglect gravitational effects within the film as they are small in comparison to surface tension effects as evidenced the low Bond number defined as

$$
B o=\frac{\rho_{l} g L_{c}^{2}}{\sigma}
$$

where $\rho_{l}$ is the liquid density, $g$ is gravitational acceleration, and $L_{c}$ is a characteristic length which for the present study is the half channel width and it is on the order of 0.5 $\mathrm{mm}$. 
The size of ultrathin films is taken as large enough to be within the continuum approximation, but small enough such that molecular forces have a significant effect on the film physics (less than $100 \mathrm{~nm}$ ) [16] compared to inertial and gravitational effects.

\subsection{Evaporation}

Evaporation is the change of phase from liquid to gas at an interface in contrast to boiling, which takes place within the entire liquid phase. The rate of evaporation can be modelled using classical kinetic gas theory to obtain Equation (2.14), known as the Hertz-KnudsonSchrage equation in the West and also known as the Kucherov-Rikenglaz equation in the post-Soviet states [12]

$$
J=\frac{2 \alpha}{2-\alpha}\left(\frac{M}{2 \pi R_{u}}\right)^{1 / 2}\left(\frac{P_{l}}{T_{l}^{1 / 2}}-\frac{P_{v}}{T_{v}^{1 / 2}}\right)
$$

where the accommodation coefficient, $\alpha$, is the fraction of molecules that change phase when meeting the interface and here for simplicity is assumed equal for evaporation and condensation. $R_{u}$ is the universal gas constant and $M$ is the molecular mass. The states $l$ and $v$ in Equation (2.14) are just above the interface in the vapour state and in the vapour bulk, respectively. A Maxwellian velocity distribution and ideal gas behaviour is assumed for both fluid states.

\subsection{Superheat}

Superheating a liquid involves increasing its temperature past its bulk saturation temperature without boiling. A superheated liquid is considered to be in a meta-stable state [17]. The superheat applied to a liquid is noted as the temperature difference between the liquid and vapour, where the vapour is saturated. 


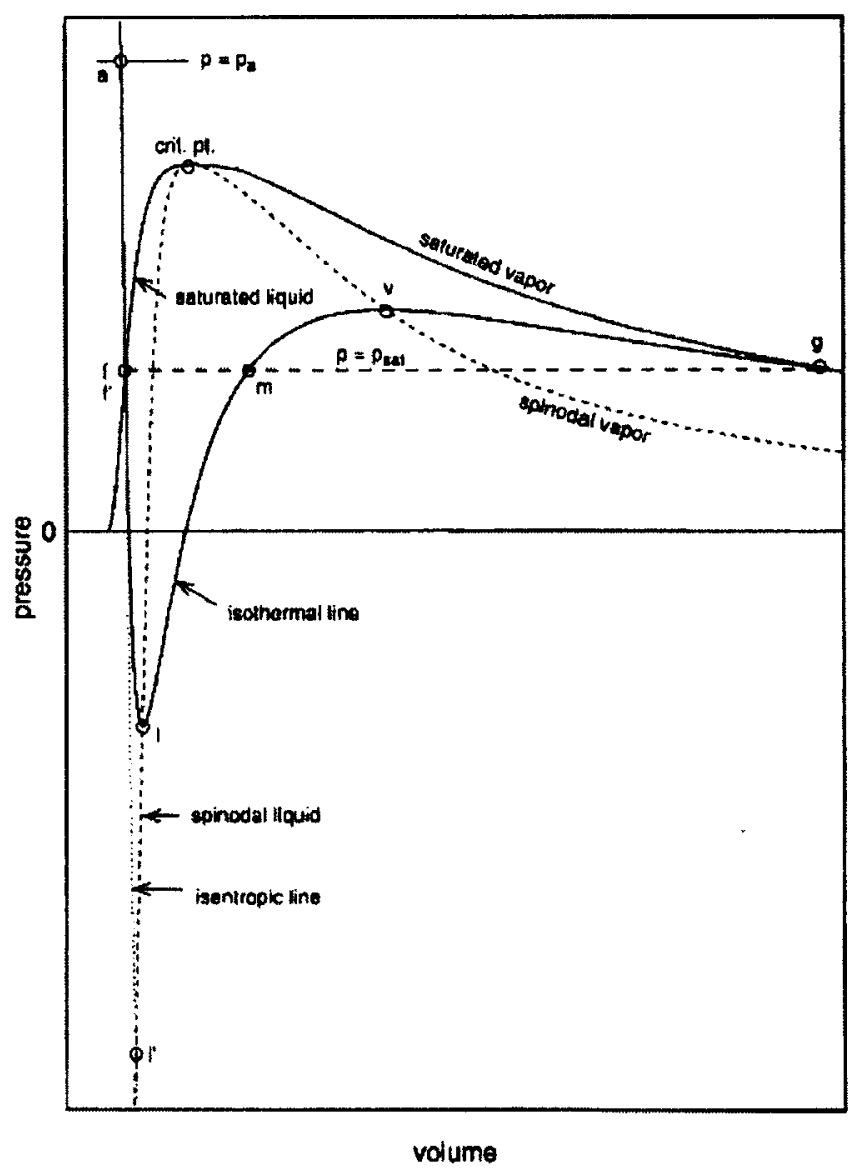

Figure (2.3): Pressure versus volume diagram with isotherm and spinodal curve to explain superheat [17]

A superheated liquid does not traditionally conform to a saturated vapour phase diagram and as such is described using an additional spinodal liquid and vapour curve shown in Figure (2.3). The region bound by the saturated and spinodal liquid curves is where a superheated metastable liquid exists in non-equilibrium since the pressure along the isotherm is below saturation. To the right of the spinodal liquid curve between points $l$ and $v$, the fluid becomes mechanically unstable as $(\partial P / \partial v)_{T}>0$. The location of onset for this mechanical instability is known as the spinodal limit [12]. This limit is not reached in this study, but rather the pressure drops only in magnitude to account for capillary and disjoining effects and thus the phase change occurs anisothermally. 


\subsection{Interfacial thermal resistance to evaporation}

The temperature at the interface does not necessarily equal that of the saturated vapour. An evaporating interface has an associated mass flux, $J$ which can be related to the temperature difference via a 'resistance' term. We can relate the two via

$$
K=\frac{T_{l v}-T_{v}}{J}
$$

where the interfacial thermal resistance, $K$ refers to the degree of non-equilibrium for an evaporating interface and $K=0$ implies that the interface and saturated vapour temperatures are equal and is known as the quasi-equilibrium limit [18]. Alternatively, $K \rightarrow \infty$ implies no evaporation for a given finite temperature difference between the interface and saturated vapour. For a finite $K$, bound by the aforementioned limits, the interface is considered to be in non-equilibrium. The temperature difference, $T_{l v}-T_{v}$ is a jump in temperature and for heated water has been experimentally found to be up to approximately 15 ${ }^{\circ} \mathrm{C}$ [19]. To estimate this resistance, Equation (2.14) can be written without pressure terms using the Clausius-Clapeyron relation as

$$
\frac{d P}{d T}=\frac{\mathcal{L} \rho_{v}}{T}
$$

where $\rho_{l} \gg \rho_{v}$ and $\mathcal{L}$ is the latent heat of vapourisation. Substituting Equation (2.14) and Equation (2.16) into Equation (2.15) and after some simplification [20], the resistance to evaporation for a fluid can be approximated via

$$
K=\frac{T_{v}^{3 / 2}}{\alpha \rho_{v} \mathcal{L}}\left(\frac{2 \pi R_{u}}{M}\right)^{1 / 2}
$$




\subsection{Stability theory}

Once an equation describing the height of the film as a function of time is determined, a small variation is applied to the height and the sign of the variation growth rate is determined. The variation with respect to the height is known as a perturbation. A negative perturbation growth rate yields a stable solution while a positive perturbation growth rate yields an unstable solution. In practice, perturbations to the film height occur naturally due to the complex nature of the flow and the underlying statistical character of matter. The motion of the fluid resulting in a perturbation is driven by the temperature difference between the solid substrate and vapour or the concentration gradient when different fluids are present.

\subsubsection{Mechanisms leading to instability}

Different physical mechanisms result in the destabilisation of an interface. Since these mechanisms are applicable to fluid systems in general, they can be generally categorised and their specific effects on evaporating thin films will be discussed.

The Rayleigh-Taylor instability results at an interface where a fluid of lower density pushes one of higher density [21]. This can be visualised by imagining the inversion of a full glass of water under standard atmospheric conditions as shown in Figure (2.4). Since the glass is rigid and the hydrostatic pressure from the column of water is much less than atmospheric pressure, the air would be able to sustain the liquid if the interface were flat. Due to naturally occurring disturbances, the interface is not perfectly flat. Thus, liquid at point $a$ is at a higher pressure than the adjacent point $b$. This pressure difference is accompanied with motion which further deforms the interface until finally atmospheric pressure makes its way to the glass ceiling and the liquid exits the glass under the influence of gravity. Orienting a capillary horizontally or upside down would enable the occurrence 


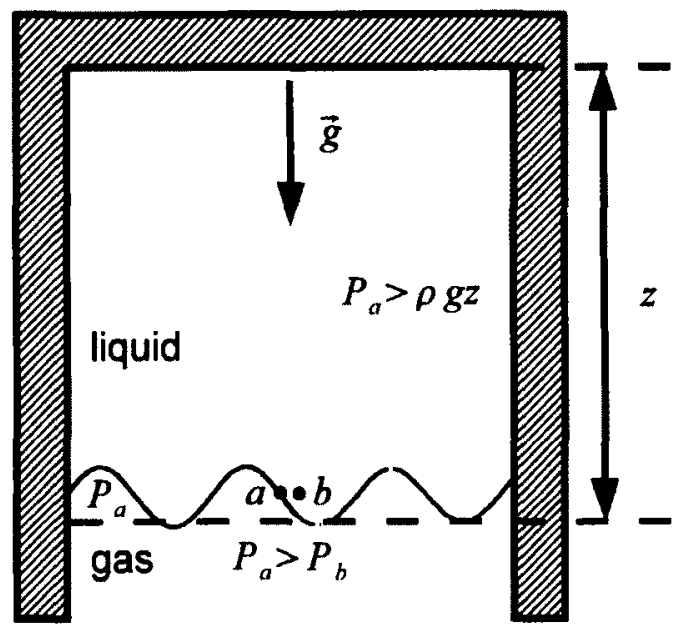

Figure (2.4): Rayleigh-Taylor instability demonstrated by the inability of a gas at higher pressure to support a liquid at lower pressure

of this instability.

The Kelvin-Helmholtz instability is a result of two fluids meeting at an interface in relative motion with one another such that they impart shear on each other [22]. Shown in Figure (2.5) is a curved interface with a subsonic gas in motion relative to the liquid phase which will result in a pressure rise at the troughs and pressure drop at the peaks as the flow expands and contracts, respectively. These pressure fluctuations will act to enlarge both trough and peak, thus destabilising the system. Surface tension counteracts the destabilising mechanisms of both the Rayleigh-Taylor and the Kelvin-Helmholtz instabilities [23]. In this study, a two-sided model is used whereby the effect of the vapour is considered negligible on the liquid. The validity of this simplification is based on the vapour viscosity and shear being much less than its liquid counterpart.

Vapour recoil instability is due to vapour molecules leaving the interface. Evaporation at the interface requires an increase in velocity of the evaporated molecule to satisfy mass conservation since $\rho_{v} \ll \rho_{l}$. If the heated interface is perturbed and assumes the shape of a wave, then liquid at the trough will tend to evaporate more readily than at the crest since it is closer to the heat source. As a vapour molecule is launched at high velocity from the 


\section{free stream gas}

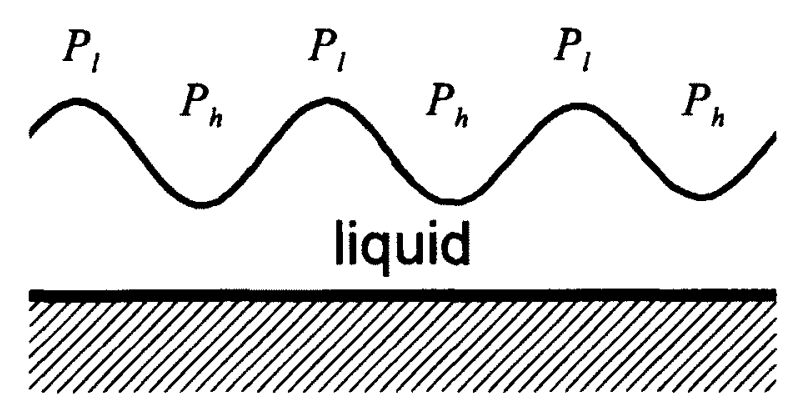

Figure (2.5): Kelvin-Helmholtz instability with high pressure at troughs and low pressure at peaks

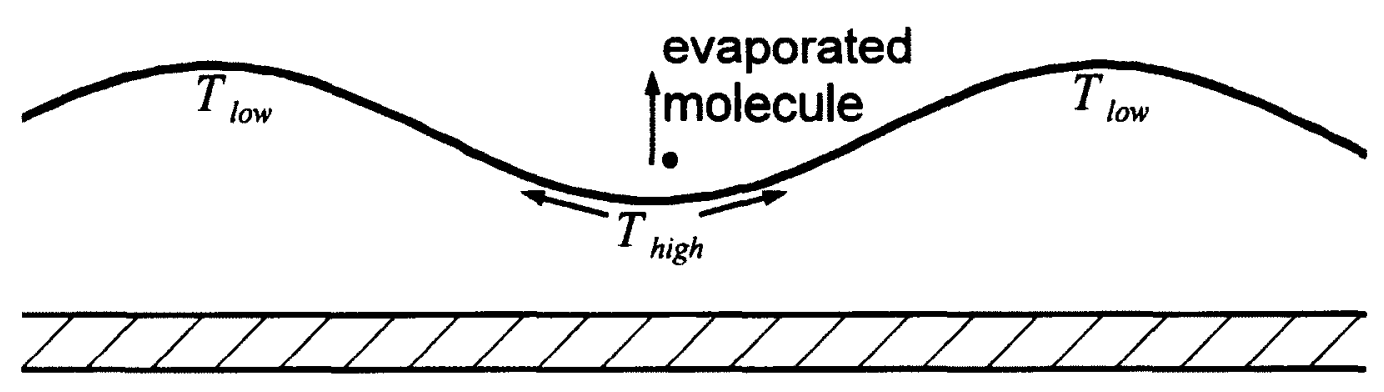

Figure (2.6): Vapour recoil mechanism amplifying perturbation by driving liquid in trough toward crest upward

interface, the reactive force felt by the liquid film tends to push liquid out and toward either neighbouring crest, thereby causing the perturbation to grow. This mechanism is illustrated in Figure (2.6). If the vapour recoil mechanism perpetuates, eventually the trough will reach the heated surface, resulting in film rupture, dryout, and degradation in heat transfer performance.

Thermocapillarity acts to destabilise the film as fluid is pulled along the interface. This effect is also known as Marangoni instability [24]. Given that surface tension decreases with temperature increase for the fluid in this study, a perturbation applied to a film will 

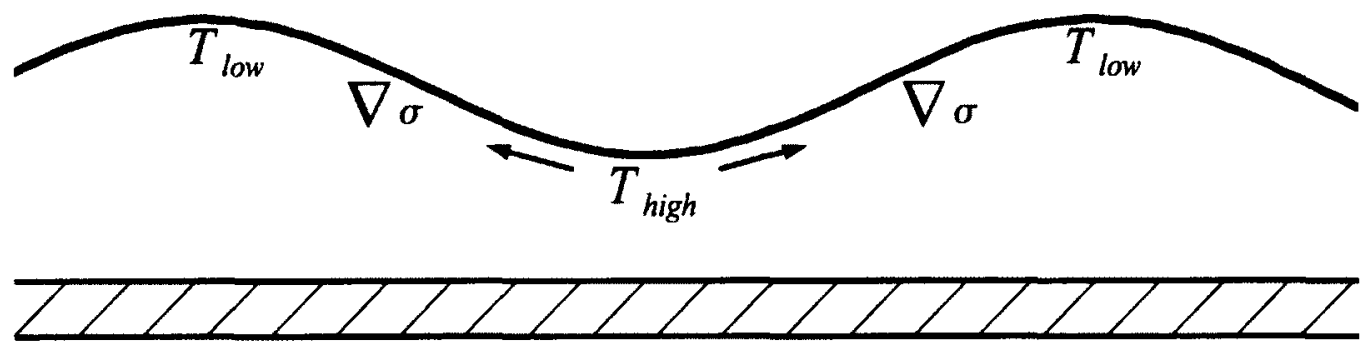

Figure (2.7): Thermocapillarity mechanism amplifying perturbation by pulling liquid from lower surface tension troughs to higher surface tension crests

result in the troughs being warmer than the crests. This results in a gradient in surface tension which pulls liquid away from the troughs and into the crests in a manner similar to vapour recoil. This mechanism is shown in Figure (2.7). Rupture instability occurs after the film becomes discontinuous. Prior to rupture, a growing perturbation at the continuous thin film interface eventually causes the liquid film to disappear at a point on the solid substrate. The vapour and solid phases are then in direct contact, as sketched in Figure (2.8). When this occurs, the flow of liquid is interrupted and the portion cutoff from the bulk does not get replenished, which leads to its depletion and consequential growth of the rupture zone. Clearly, this is detrimental to cooling applications as a hot spot forms where rupture occurs and heat transfer becomes more unpredictable due to this additional discontinuity.

Rayleigh-Bernard instability arises if heat is applied to a liquid layer from below which causes the proximate warmer liquid to rise and the colder distant liquid to fall [23]. As this process evolves, convection cells can form if gravitational effects overcome the viscous damping of the liquid. Given that heat is applied from above for testing in this study, this instability is less likely to form than if heating from below. Possible instability could still occur if evaporative cooling creates a significant vertical density gradient. 


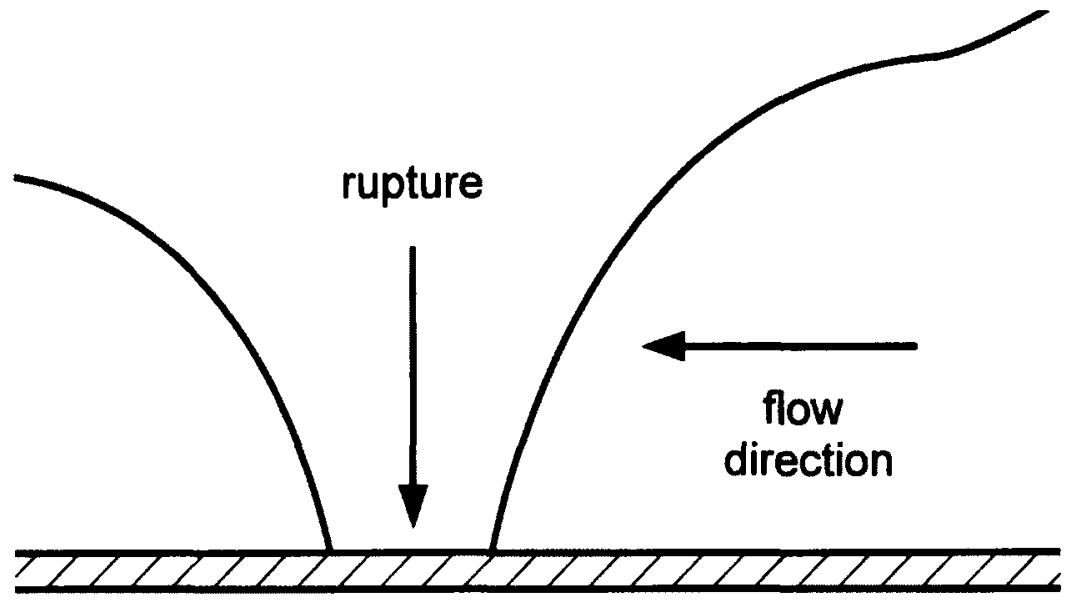

Figure (2.8): Thin film rupture

\subsection{Relevant previous research}

A brief overview of theoretical and experimental interfacial stability research is now given. Before the study of thin film stability was specifically undertaken, the stability of relatively large and flat liquid-vapour interfaces was studied due to ease of their observability.

Since the work of Young and Laplace in 1805, characterising the meniscus and its behaviour has been an endeavour that continues even today [25]. The meniscus has been studied in the context of the interface between neutrally buoyant fluids such a soap bubble in air. According to [25], experimental work by Plateau in the late 19th century focused on the stability of soap films made into different geometries and neutrally buoyant immiscible liquid combinations forming a meniscus. Adding complexity, one can study the meniscus which bounds two fluids of different densities such as a liquid and gas. In [25], it is also mentioned that work by Duprez in the mid-19th century showed the effect of capillary pressure on density inversion by decreasing the initially higher density of a fluid supporting another fluid until the density of the supportive fluid was less than the one above. The ability of a low density fluid to hydrostatically support a high density fluid was attributed to the surface tension at the interface. Adding further complexity, gravitational or electrical 
force fields can be considered. Studying the stability of hanging or pendant drops was undertaken throughout the 20th century by various researchers. For a constant contact angle (between the liquid and substrate) pendant drop, it was found that increasing the volume destabilised the hanging drop against gravity [25].

To be more specific, we now focus on thin film stability since it is the subject of this study. We first define what is meant by thin in the context of thin film research. An ultrathin film is defined as small enough that intermolecular forces are significant, but large enough to satisfy continuum mechanics (a few molecular diameters to $100 \mathrm{~nm}$ ). Liquid inertia and gravitational effects on nearly horizontal films are considered negligible. Inertia does become significant before film rupture as the liquid moves more rapidly. An area of rupture is defined as a location where the film thickness is null. An ultrathin film can form from liquid flowing down a wall such as in a thermosyphon or from any other heated substrate where evaporation is occurring.

Although thin films are theoretically capable of convecting heat at significantly high transfer coefficients since a very small temperature drop occurs across the film height, practically there is a tendency for the film to rupture and dryout [16] leaving a potentially damaging hot spot for the device being cooled. Surface wave linear theory in the 1950s and non-linear theory in the 1960s was developed for the stability of films of various thicknesses. In the 1970s, the additional stress normal to the interface due to the phase change was considered. The use of lubrication theory to model a thin film has been used extensively as variation of dependent variables is more prominent in the direction normal versus parallel to the wall.

The work described in [26] involves solving for the stability of a flat thin film for molten metals where the Prandtl number, $\operatorname{Pr} \ll 1$ allowing for a solution in terms of powers of $\operatorname{Pr}$. It was found that the effect of vapour recoil was more significant on metals than for fluids like water or ethanol. 


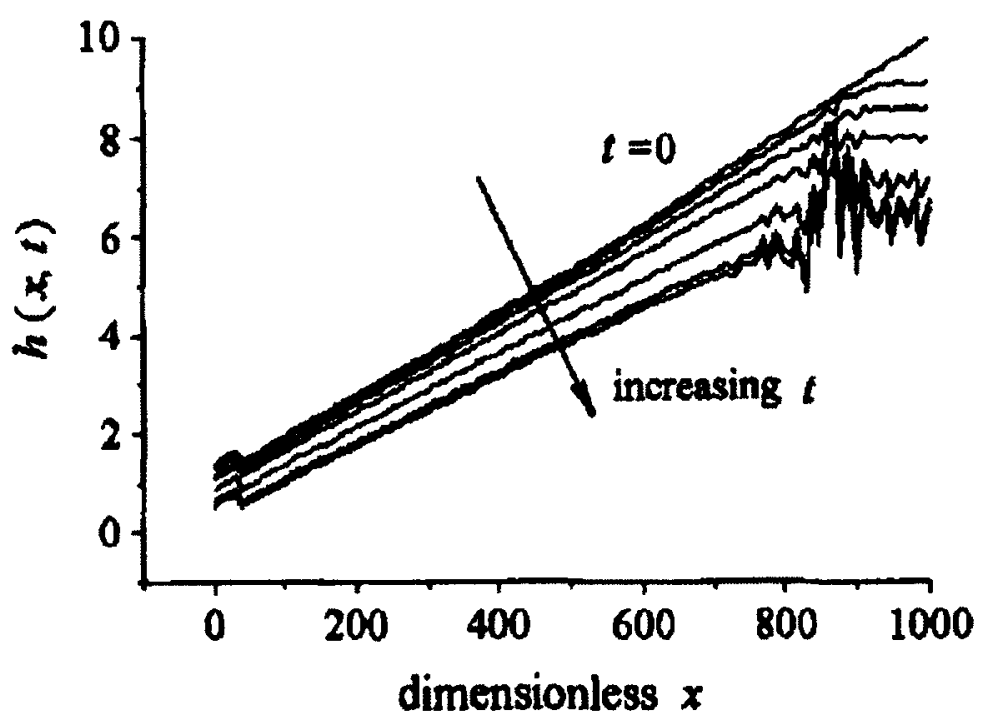

Figure (2.9): Linearly increasing thin film evolution with instability originating in thicker film portion [27]

When describing the thin film profile and how it changes in time, its representative form is often referred to as the 'evolution equation' as derived in [18] for an initially flat film.

An alternative evolution equation was used by Lichun et al. [27] for a capillary tube accounting for curvature in cylindrical coordinates. A numerical solution was obtained by finite difference methods. For a linearly increasing film, it was found that when a perturbation was applied, instability originated in the thicker region and its effect would travel toward the thinner region which was the first to rupture given its vicinity to the wall. The pattern of this instability is shown in Figure (2.9). Rupture time was found to decrease with increasing capillary tube diameter. It was concluded that the stabilising effects of capillary pressure in the bulk region delayed destabilisation and consequential rupture in the thin film region.

Given the cohesive tendency of a liquid-vapour interface, a small enough interfacial perturbation at one point will not create a discontinuity in the surface. The elastic nature of a surface will instead distribute the energy of a disturbance perturbation as wave motion 
[28]. Depending upon conditions, the waves can become periodic or chaotic and they can cause the film to rupture.

Perturbations of a known frequency were applied to a falling liquid film on an incline [29]. Waves were observed at the interface using fluorescent imaging. As a light was shone onto the film, the resulting fluorescence from the surface was filtered from the source having a different wavelength. The relative thickness of the film was determined with a sensitivity of up to $1 \mu \mathrm{m}$. Two-dimensional and three-dimensional instabilities were found to occur at lower and higher Reynolds numbers, respectively.

The complexity of the free boundary and non-linear nature of thin film stability can be simplified by considering long-wave effects. Given that change along the film is more gradual than normal to the film, the length scales are separated. Long-wave theory was used to reduce the governing equations and boundary conditions to their leading-order terms. Long-wave theory assumes disturbances have a large wavelength in the context of the thin film length. Near rupture, long-wave theory thus becomes invalid. The comparison of long wave-theory to direct numerical simulation was found to agree qualitatively, except close to rupture according to a heated, non-evaporating, initially flat film numerical study performed using an arbitrary Lagrangian-Eulerian finite element method to track the free interface in time and space [30]. When accounting for thermocapillarity in the interfacial analysis, it was found to have a destabilising effect on a corrugated heated liquid-vapour interface. Higher surface tension at colder crest would pull and viscous effects would deplete the trough making it susceptible to rupture. Interfacial temperature variation does not significantly affect time to rupture for low viscosity fluids, but does so for ones with higher viscosity and temperature differences. Accounting for a variable temperature at the interface reduced the time to rupture. The vapour recoil mechanism was found to be destabilising for both evaporating (condensing) films on a heated (cooled) solid substrate [30]. For the case of film boiling, vapour recoil is stabilising as it tends to reduce the interfacial 


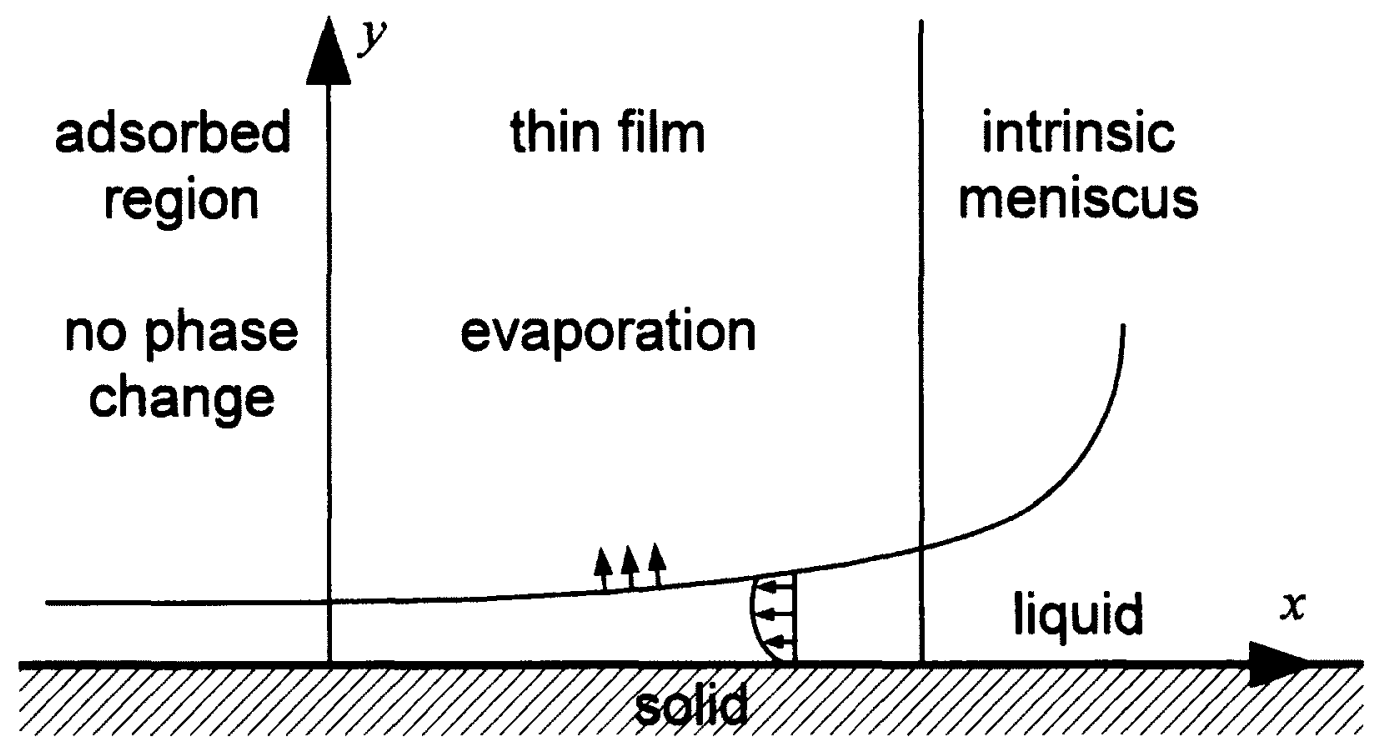

Figure (2.10): Thin film as part of meniscus

wave amplitude and to counteract Rayleigh-Taylor instabilities. In general, vapour recoil is negligible unless heat fluxes are very high [30].

The thin evaporating film sketched in Figure (2.10) is physically part of an extended meniscus in a vertical capillary tube. The term 'extended' refers to the continuation of the meniscus profile past the bulk region on the right-hand side of Figure (2.10) into thin film region and ending in an absorbed layer as opposed to ending at a point extrapolated from the intrinsic region to where the solid, liquid, and vapour regions meet. The thin film is geometrically distinguishable from the adsorbed layer and intrinsic or bulk region in that its change in height with respect to length is greater than the adsorbed region where $h_{x}=0$ and less than the bulk region where $\kappa_{x}=0$.

Modelling the behaviour of the entire system can thus be useful to distinguish the effects occurring on the microscopic film level and those on the macroscopic bulk level. The modelling of an evaporating meniscus in a capillary tube was performed [31]. The balance of capillary rise, gravitational pull, viscosity, and inertia dictates how a meniscus will behave within a tube. Oscillatory behaviour within the tube was found to be a function of 


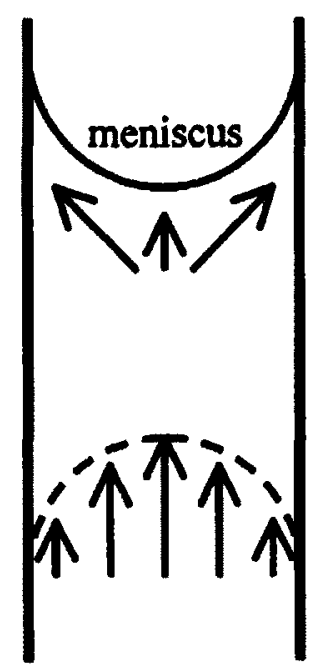

Figure (2.11): Meniscus capillary rise [32]

the tube's diameter and the liquid's viscosity. A meniscus residing within a larger diameter tube was found to be more susceptible to oscillations. Vapour recoil and gravity tended to push the meniscus down, while capillary pressure pushed it up. Viscosity acted to dampen the meniscal motion.

The experimental capillary rise dynamics within an unheated capillary tube has been studied in [32]. Capillaries with radii from 0.1-0.5 mm were tested with both diethyl ether $\left(\mathrm{C}_{2} \mathrm{H}_{5}\right)_{2} \mathrm{O}$ and n-dodecane $\left(\mathrm{C}_{12} \mathrm{H}_{26}\right)$. The capillary height was observed using cameras once contact was made with the liquid reservoir. Comparison with a model similar to [31] was performed. The model assumed Poiseuille flow in the capillary as illustrated in Figure (2.11). Since the actual flow within the capillary is more complex, given its radial component, work still remains for accurately modelling meniscal motion in a capillary tube.

A heated ethanol meniscus enclosed in a glass test cell open to atmosphere was observed in [33]. Photography was used to capture the meniscus profile. Heating was accomplished using a nickel vapour deposited resistive element. Temperature was measured using 
thermocouples. A cathetometer was used to measure the height difference between the capillary and reservoir. The pressure drop between the reservoir and a point downstream was calculated using a manometer height difference. The pressure change due to evaporation was approximated by relating the change in curvature of the meniscus between its heated and unheated form to the pressure drop using the Young-Laplace equation. This introduces some error as the equation is based on a fluid in equilibrium [34]. Nonetheless, by assuming Poiseuille flow within the tube, an average velocity was obtained and then related to the heat input and curvature. Accounting for heat transfer to the tube alone was accomplished by measuring the temperature difference between the tube and ambient by heating without any liquid inside the tube. The height of the capillary and meniscus shape were found to be dependent on the evaporation rate since the pressure field within the meniscus varied with the evaporation rate. The heated meniscus was seen to be stable throughout testing. Stability seen in this work is consistent with the author's experience with testing in ambient which is much less prone to instabilities compared to testing in a single fuid environment.

A numerical study of an evaporating $n$-heptane $\left(\mathrm{C}_{7} \mathrm{H}_{16}\right)$ meniscus in a channel residing in ambient has been performed [35]. The shape of the meniscus was taken from experimental work. As such, the model described in [35] neglects the effects of disjoining pressure since the effect was small on the larger observable experimental length scale. A mesh was created and solved numerically using Fluent given a pressure field as a boundary condition for the liquid incoming from the bulk region. Results showed a high interfacial heat flux close to the contact line which is indicative of the effectiveness of the thin film in transferring heat.

Infrared temperature measurements of a two-dimensional heated liquid $\mathrm{n}$-hexane film surrounded by air was performed by the researchers of the previous study [36]. The meniscus was created by horizontally spacing two circular flats and filling the internal space with a syringe pump through the top flat as shown in Figure (2.12). Heating was provided by 


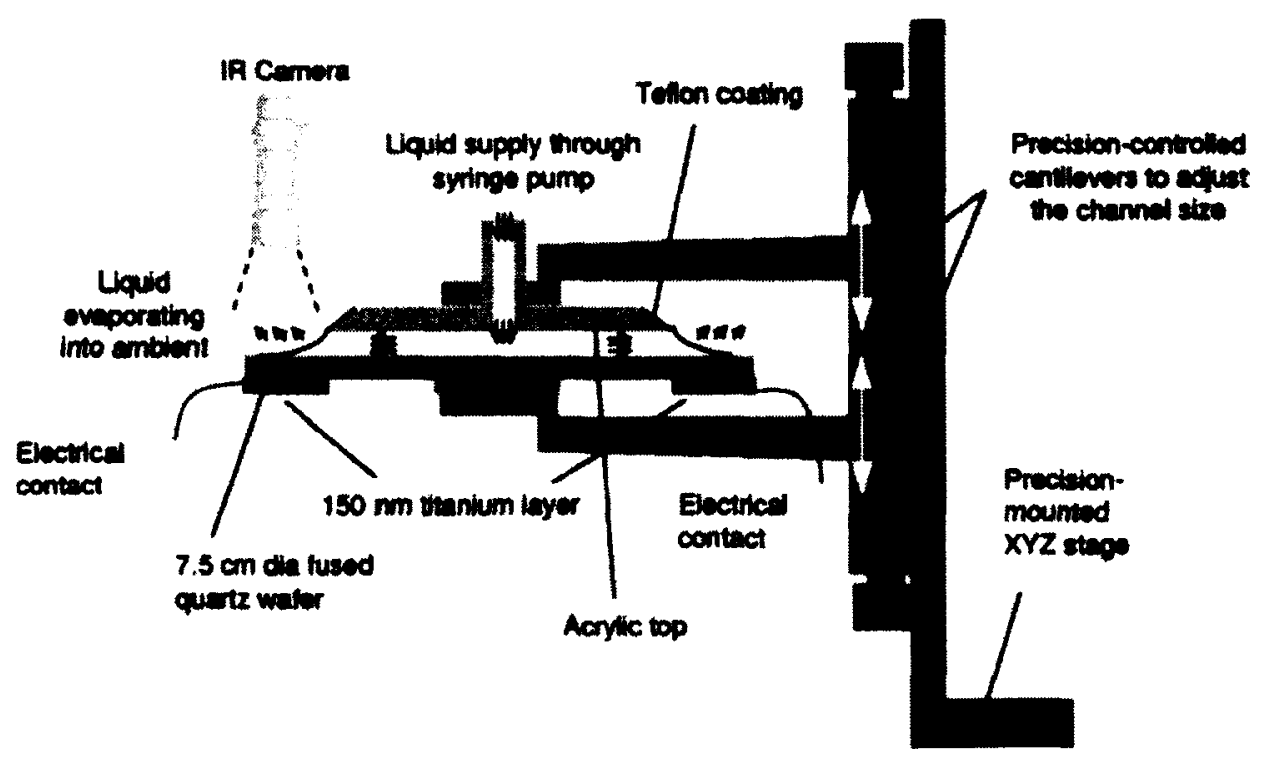

Figure (2.12): Heated film test setup with infrared observation camera [36]

a deposited titanium annulus. The sessile meniscus was formed on top of a quartz wafer while meniscus formation was inhibited on the top acrylic flat by chamfering the edge of the top flat and adding a Teflon hydrophobic coating. By applying a constant heat flux and changing the evaporation rate via the pump flow rate, the meniscus shape was seen to vary. A higher evaporation rate resulted in an increased interfacial area by elongating the meniscus, which makes sense since this is where evaporation occurs. The change in channel width from $500 \mu \mathrm{m}$ to $600 \mu \mathrm{m}$ was shown to have little effect on the meniscus profile when varying the flow rate and heat flux. The measured temperature was plotted along the film cantered about the contact line for both above and below the wafer on which the film resided. It was found that the lowest temperature above the wafer occurred at the contact line. This implies that the region closest to the contact line is more effective in removing heat than a similar region within the bulk meniscus. The mean temperature profile of the wafer was used in a one-dimensional model in order to predict the heat transfer in a $50 \mu \mathrm{m}$ region close to the contact line where the infrared camera resolution was too low to capture 
sufficiently accurate data. It was estimated that approximately $70 \%$ of the heat was dissipated through this region which indicates the importance of this phase change mechanism that occurs within the thin film and warrants the study of its stability.

The use of thermochromic liquid crystals (TLCs) to measure the temperature near the triple line of an evaporating meniscus in a capillary tube was studied by Buffone and Sefiane [37]. Although TLCs can detect or respond to a wide temperature domain compared to other temperature sensors, they are inherently susceptible to chemical contamination, radiation, and oxygen. Calibration is essential for quantitative results and testing must be performed shortly thereafter due to the rapid TLC degradation (from a few minutes to a couple hours). Sealing of the TLC does extend its effective life, but doing so in the small scale near the interline also blocks critical details. A calibration bar with an imposed temperature difference along its length was used and thermocouples were positioned lengthwise to relate temperature readings to colour. A horizontally oriented $1.63 \mathrm{~mm}$ inner diameter glass capillary tube, initially filled with n-pentane but not subsequently replenished, was used for testing. It was reported that n-pentane wetting of the TLC resulted in destruction, thus making its use impractical in a chamber saturated with n-pentane.

The use of infrared (IR) thermography is relatively non-invasive and robust compared to TLC thermography. Previous studies have used IR thermography to observe the interface inside capillary tubes between $0.6-1.63 \mathrm{~mm}$ in diameter with a $30 \mu \mathrm{m}$ spatial resolution and a $20 \mathrm{mK}$ temperature sensitivity [38]. The temperature profile near the triple line was observed and it was concluded that more evaporative cooling occurred there compared to the central bulk region of the interface.

An experimental study of the convection within a capillary pore containing a volatile evaporating fluid was performed in [39]. Seeding particles inside the liquid were used for visualisation of the convection roll adjacent to the interface as shown in Figure (2.13). The tracer particles used were made of $20 \mu \mathrm{m}$ diameter nylon spheres. Borosilicate glass 


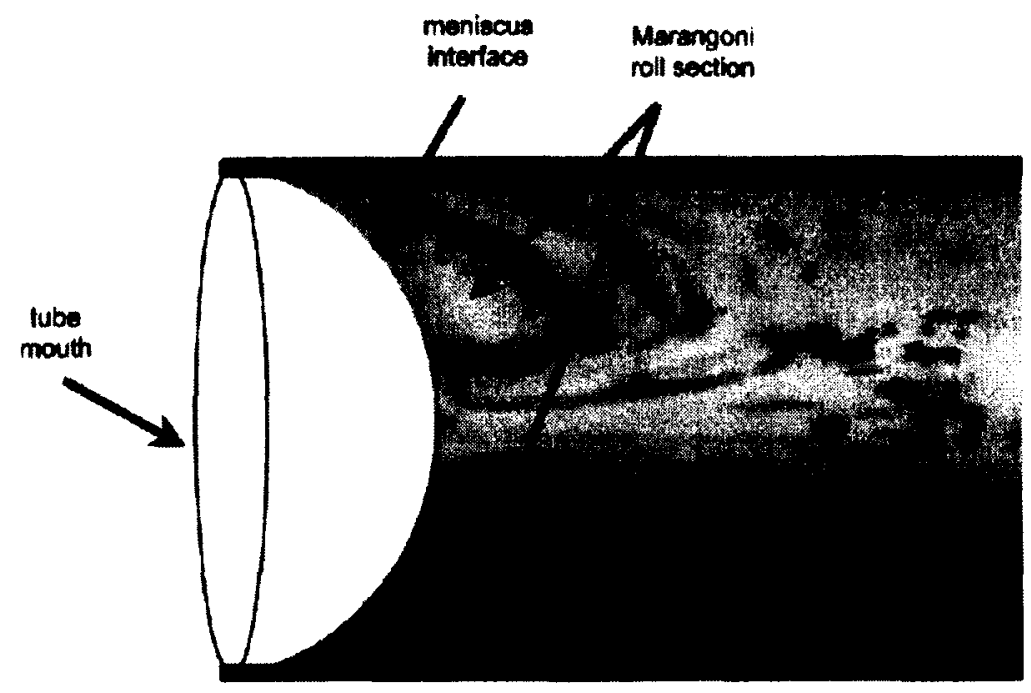

Figure (2.13): Convection (Marangoni) rolling near interface visualised using seeding particles [39]

particles under $26 \mu \mathrm{m}$ in diameter were used for particle image velocimetry (PIV) measurements. Care was taken to keep the capillary pore bubble free as they tended to cause significant variation in the results. Evaporation was sustained without heat addition and only relied on the volatility of the working fluid in ambient. It was found that the evaporation rate decreased as the meniscus receded into the tube during the experimental progression since the vapour concentration gradient and corresponding diffusion would decrease. The evaporation rate was seen to increase with increasing pore size due to an increasing surface area. It was found through infrared imaging that evaporation was greater near the wall compared to the bulk region of the interface. Since the evaporation rate along the interface was different, so was the temperature, and a surface tension gradient came about due to the temperature gradient and Marangoni convection cells ensued.

Heated capillary tube testing was performed and the thermocapillary convection was also measured by these researchers [40]. A silver heater coating was applied to the substrate surface and could provide up to $2 \mathrm{~W}$ of power on a capillary of $0.6 \mathrm{~mm}$ internal diameter with a $0.1 \mathrm{~mm}$ wall thickness. Power measurement was accomplished using a 

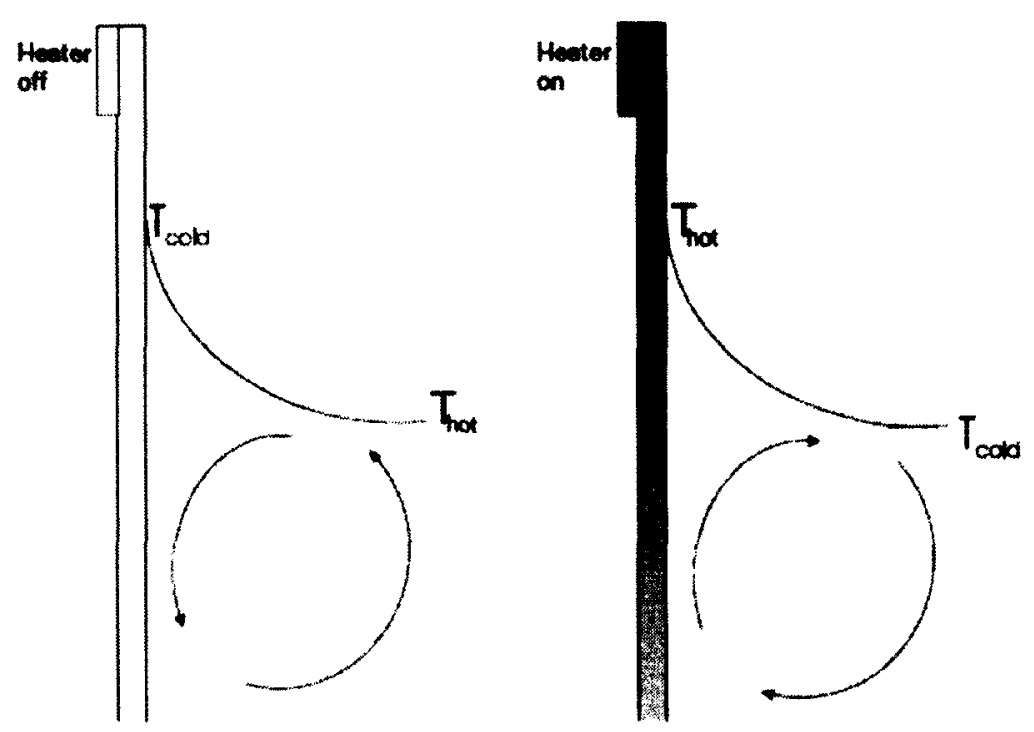

Figure (2.14): Convection rotation reversal when reversing temperature difference along the interface [40]

voltmeter and amp meter. It was found using $\mu$-PIV that the rotational direction of convection would reverse when the temperature difference between the bulk region and the film was reversed as sketched in Figure (2.14) and is attributed to a change in direction of the surface tension at the interface. Heat was applied from both above and below the meniscus. Heating from below did not alter the position of the meniscus with respect to the tube axially as it remained hinged on the edge. Boiling was observed after applying sufficient heat from below. There was also no flow reversal of the convection roll when heating from below because boiling was reached before the necessary temperature difference was established. Alternatively, heating from above would alter the position of the meniscus along the wall and the convection roll direction reversal did occur with increased applied heat. An IR camera was used to obtain the temperature profile along the tube. Matte black paint was applied to the tube in order to minimise reflections sensed by the IR detector. IR imaging was accomplished in darkness, making manual adjustments of the meniscal position practically unrealistic as the meniscus moved. Unheated testing revealed a lower temperature along the interface close to the wall compared to near the bulk, suggesting higher evaporative 


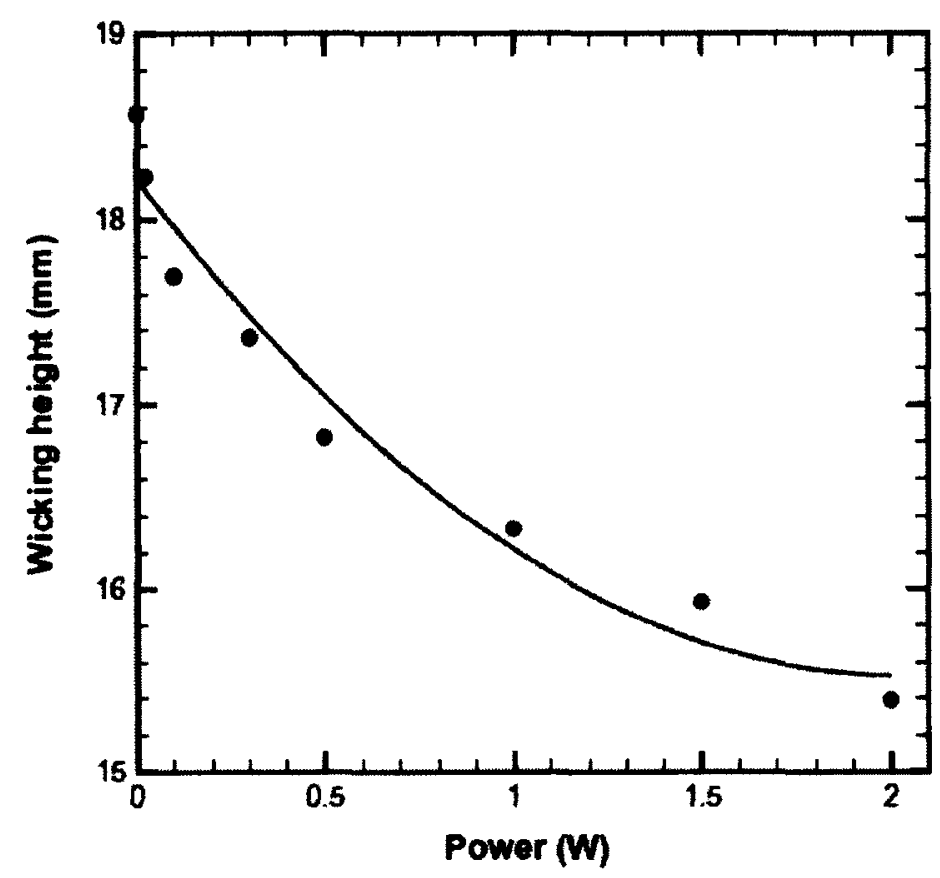

Figure (2.15): Capillary height variation for a methanol meniscus with applied power [41] cooling near the wall.

Further study of a heated meniscus inside a capillary tube was experimentally performed in [41] to gain insight into the thin film behaviour. Limitations in the resolution of infrared imaging (8-12 $\mu \mathrm{m})$ meant the thin film near the triple line could not be analysed accurately since its entire length was roughly on the order of $0.1 \mu \mathrm{m}$. The spreading of heat through the glass tube wall also prevented accurate determination of the temperature profile at the thin film.

The capillary height of a methanol meniscus as a function of power was measured and plotted in Figure (2.15). The capillary height was used to estimate the contact angle and to gain insight into the triple line region.

Experimental work by Sefiane's group [42] found an evaporating meniscus in a capillary tube to have macroscopic oscillatory behaviour in both the interface and meniscus body at frequencies of $5 \mathrm{~Hz}$ and $1.4 \mathrm{~Hz}$, respectively. Variable evaporative cooling at the 
interface was determined to be the cause of the instabilities. Oscillations were found to coincide with the convectional rolling within the meniscus as evidenced by tracer particles used in $\mu$-PIV measurements. Observation was not so clear as to the origin of these instabilities and further experimental work was warranted.

Comparing unheated evaporating menisci in capillary tubes of different diameters in [43], it was found that the smaller diameter tubes were more prone to oscillatory behaviour. At first glance, this seems to contravene the idea of surface tension acting as a stabilising agent. Instead, the smaller diameter tube was reasoned to harbour larger surface tension gradients due to the smaller distance between the wall and central meniscal bulk. Evidence of a significant fraction of evaporation occurring in the thin film region comes from the observation that the evaporation rate of the meniscus was found proportional to its diameter as is the perimeter and not the square of the diameter as is the surface area.

The evaporative enhancement of interfacial vapour recoil in a partial vacuum was experimentally studied by [44]. A test apparatus made of glass was mechanically evacuated using a pump. A liquid reservoir was heated via current running through a nichrome wire coiled around the outside reservoir. Fluid cleanliness was obtained via a continuous distillation process. Cooling to promote condensation was achieved by forced air. A dropper was used to estimate the evaporation rate by counting drops of condensate flowing through the chamber wall over time. A plumb residing in a liquid reservoir was moved to control the interface position. The vapour recoil mechanism caused by the velocity increase of evaporating fluid as its density decreases to satisfy mass continuity across the interface results in a reaction force on the interface toward the liquid and a change in shape of the interface. Since temperature variations occur across the interface, localised recoil results in relative motion of different parts of the meniscus with respect to itself. A triethanolamine $\left(\mathrm{C}_{6} \mathrm{H}_{15} \mathrm{NO}_{3}\right)$ fluid environment with variable pressure was used to observe the interface. To isolate the effect of vapour recoil on the system, a surfactant was added to the interface. The 
surfactant ( $\alpha$-tocopheral acid succinate) gave the fluid interface a greater elasticity and thus inhibited localised meniscal activity. The primary mechanism associated with evaporation for the surfactant laden interface was natural convection between the vapour at the interface and neighbouring bulk vapour. It was found that minute amounts of surfactant would dampen the effects of vapour recoil readily, so a clean fluid was imperative for the onset of the vapour recoil mechanism. Evaporation rates were measured along with bulk and saturation temperature for a given pressure. The superheat was found to be much greater and the evaporative flux lower for the interface with the surfactant suppressed vapour recoil compared to the clean interface. Temperature across the thermal boundary layer above the interface was measured using a fine thermocouple and the layer size was found to be on the order of $1 \mathrm{~mm}$. Heating from different locations was performed and it was found that asymmetric heating had little impact on the results. The heat transfer coefficient was found to be higher with lower superheat (higher evaporative mass flux) as shown in Figure (2.16). This was attributed to the vapuor recoil mechanism since more evaporation and a larger recoil caused more mixing and enhanced the heat transfer mechanism. The heat transfer coefficient was also found to increase with increase in pressure for low pressures $(<7 \mathrm{~Pa})$ since the absence of a continuum at lower pressures meant viscous shearing, which promotes evaporation, was unsustainable. For greater pressures $(>7 \mathrm{~Pa}$ ), the heat transfer coefficient would then decrease. At higher pressures, the vapour recoil was lower and as such so was the heat transfer coefficient. Although vapour recoil does play a role in meniscal stability, it was not considered significant for the fluid setup in this study since the vapour pressure is significantly higher.

Experimental thin film research by [45] used interferometry to measure the film height within less than $2 \mu \mathrm{m}$. The setup consisted of an evaporating n-pentane corner meniscus enclosed in a quartz cuvette having a rectangular cross section as shown in Figure (2.17). The closed cuvette was filled with n-pentane liquid and nitrogen gas, allowing for the vapour 


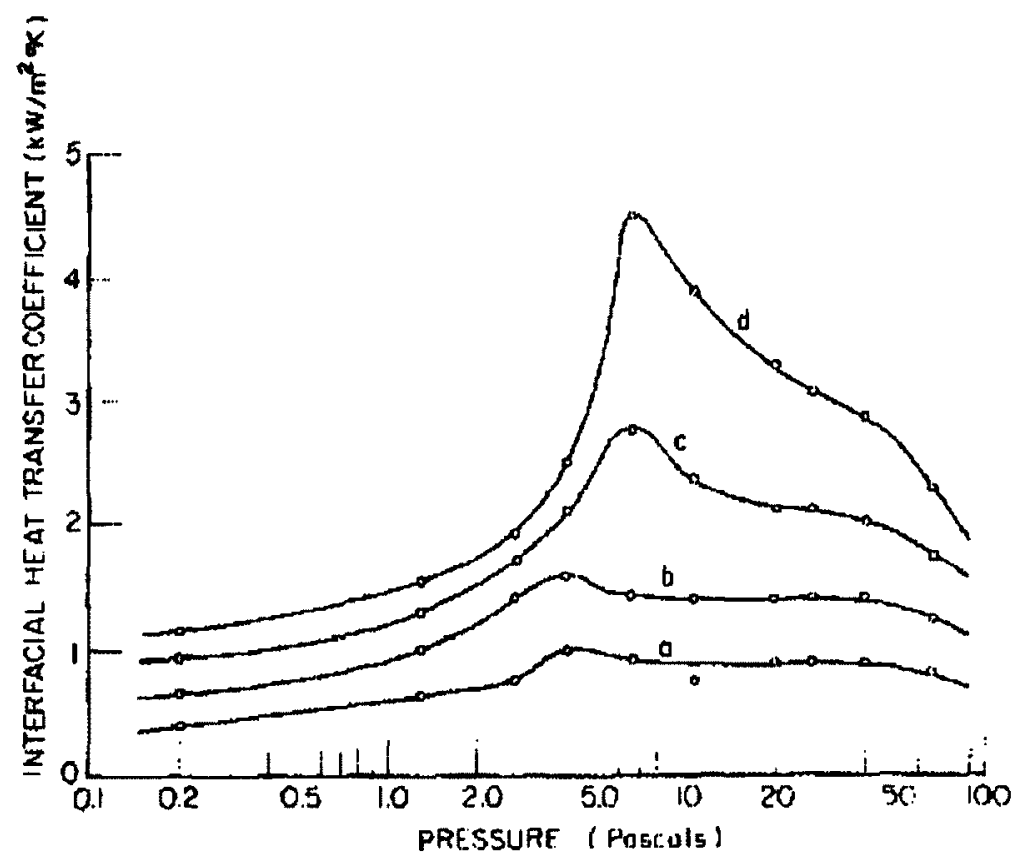

Figure (2.16): Average heat transfer coefficient variation with temperature. Mass flux a) 10 , b) 15, c) 20, d) $25 \mathrm{gm}^{-2} \mathrm{~s}^{-1}[44]$
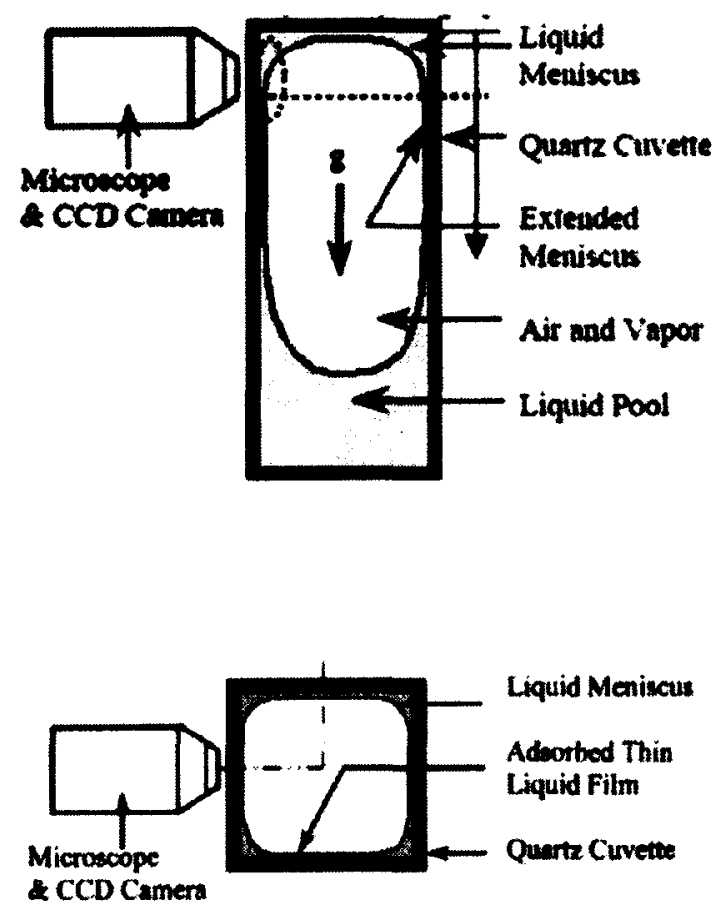

Figure (2.17): Experimental setup of a $3 \mathrm{~mm} \times 3 \mathrm{~mm} \times 43 \mathrm{~mm}$ heated cuvette viewed parallel and normal to gravity [45] 
space to be composed primarily of n-pentane at atmospheric pressure with some relatively inert nitrogen gas. Heat was applied electrically from above and the base was sealed using a Teflon plug. The outside of the cuvette acted as a fin for cooling the closed system. Temperature was measured along the length of the cuvette using thermocouples. The cuvette was positioned with respect to the microscope using three orthogonal linear stages. Vibration isolation was accomplished using an air spring to support the apparatus. The interference fringe patterns were captured using a charge-coupled device (CCD) camera and the velocity of the meniscus normal to the wall was calculated by observing the time change in the interference patterns. Meniscal thicknesses with a resolution of $0.5 \mu \mathrm{m}$ were measured and noise was filtered using polynomial fitting. Higher-order polynomials were used to curve fit data as the film thickness decreased. Curvature was calculated using the film thickness profile and was seen to vary between isothermal and non-isothermal test cases. The pressure difference across the interface was calculated using the augmented Young-Laplace equation to account for disjoining pressure effects. Pressure jumps in the $\mathrm{kPa}$ range were calculated based on the experimentally derived film thickness. Evidence of the suction effect toward the contact line of disjoining pressure was found.

The stability of a n-pentane meniscus in a capillary tube within a saturated n-pentane environment was studied by Pratt [46]. The temperature difference across the meniscus inside different tubes was measured using thermocouples embedded in the glass tube. Heat was applied using a nichrome wire in an incremental fashion until the meniscus could no longer sustain the load. The temperature difference at the maximum heat load was recorded. A theoretical criterion was developed to predict the minimum superheat which could destabilise the meniscus. The challenge with determining a realistic criterion was knowing the actual thin film profile in order to quantify the parameters comprising the criterion. Additionally, the scaling used to obtain the criterion instilled the requirement for choosing a characteristic height for the thin film. The choice for the characteristic length 
should reflect the physics which relates to systematic stability in some way in order to have predictive ability.

\subsubsection{Conclusions}

Research into understanding the behaviour of a thin evaporating liquid film is very difficult as there are still many unresolved issues. Firstly, the shape of the film is not well known given that the small length scale can not be resolved using an optical microscope. The addition of particles in PIV to enhance imaging can increase the resolution, but at the cost of additional impurities and energetic excitation of the film as smaller wavelengths are used for more detailed observation. Realistically, the current practical measurement accuracy is on the order of a micrometer while theory dictates a nanometric length scale. One issue inherent to our theory involves the difficulty with accurately quantifying evaporation using kinetic theory as there are deviations from experimental findings [47]. In addition, given that the form used for disjoining pressure assumes three distinct (solid, liquid, gas) phases, it does not account for evaporation which would have an effect on the intermolecular interaction. Finally, many contemporary works treat the entire film domain as a continuum, which is realistic in the bulk region, but loses validity as one approaches the adsorbed layer. Despite the current limitations, it is important to persevere in our experimental observation and theoretical description of the evaporating thin film given the numerous beneficial applications described in Chapter 1. 


\section{Chapter 3}

\section{Experimental setup}

\subsection{Overview}

In order to initiate and observe the destabilisation of a single pore meniscus in a heated capillary tube, an experimental setup was developed at Carleton University. The setup described by Pratt in [46] was used to guide the experimental setup development in this study. The developed setup, shown in Figure (3.1), is briefly described below and in Appendix A with greater detail emphasising the following:

- Vibrational impact management associated with observing a microscopic fluid system

- Positioning the chamber with respect to the microscope linearly in three orthogonal directions and rotationally about the vertical axis

- Preliminary ambient testing as a developmental tool for vacuum testing

- Optics to observe meniscal behaviour

- Temperature, pressure, and event recording using vacuum feedthroughs 
- Fixing the meniscus in space with respect to the chamber by varying the reservoir position with respect to the capillary tube

- Scaling the magnitied image

- Controlling the fluid environment surrounding the capillary tube

- Manufacturing thin and thick walled capillary structures with heating and temperature sensing capability

- Cleaning the capillary tubes for consistent testing

- Insulating the heater and cooling the reservoir

A massive granite block and rubber matting were utilised as a test base to mitigate the effects of external vibrations during testing. Glass beads were placed in the liquid reservoir inside of the chamber to dampen liquid motion near the capillary tube.

An ambient test setup open to atmosphere was first developed consisting of an acrylic enclosure to diminish the effects of laboratory air currents. A vacuum setup was subsequently developed to evacuate the air surrounding the capillary test tube for testing in a saturated n-pentane environment at room temperature.

A microscope with two eyepieces was used to optically observe the meniscus. A reticle in one eyepiece was used for coarse adjustments. A camera was placed in the second eyepiece to monitor and record the finer movements of the meniscus during testing.

Positioning of the test setup with respect to the microscope was made possible using a horizontal compound slide and rotary table. The microscope was vertically adjustable and could be moved normal to and rotated about the vertical axis.

Two sets of round capillary tubes with comparable inner diameters were tested. Tube wall thickness was used to distinguish one set from the other. A heater was deposited at the 


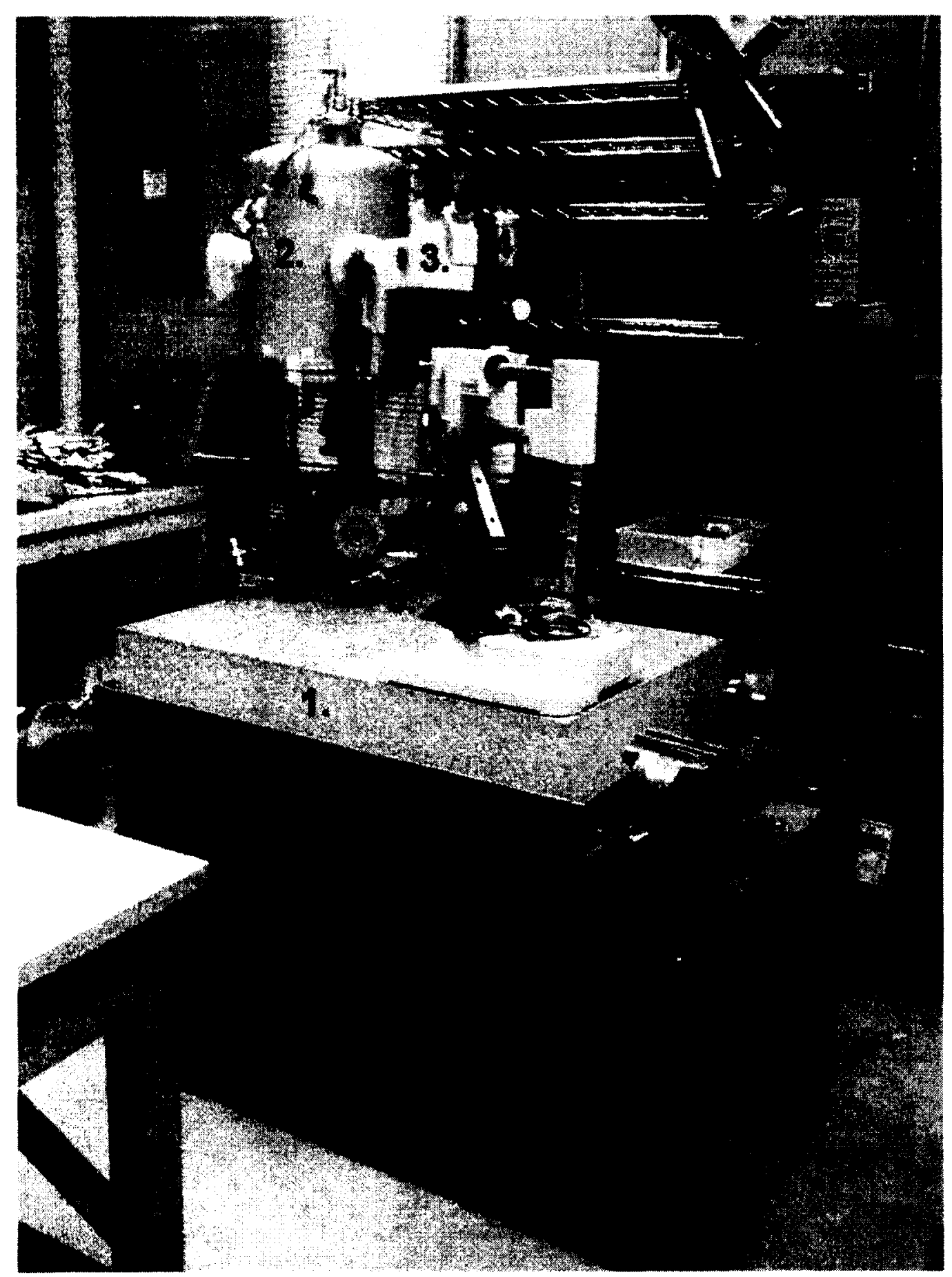

Figure (3.1): Test setup: 1. test base, 2. chamber, 3. microscope, 4. external reservoir, 5. bell jar crane, 6 . chamber positioning control 
top of each capillary tube. Heater voltage and current were measured during testing. Tube temperature was measured using two thermocouples placed below the heater along the tube axis. Thick tube thermocouples were imbedded inside holes drilled into the tube wall and thin tube thermocouples were epoxied directly onto the outer tube wall. The meniscus was positioned axially in between the thermocouples. The inner wall at the same axial position as the top thermocouple was in contact with vapour above the meniscus and the inner wall at the same axial position as the bottom thermocouple was in contact with liquid below the meniscus. The test capillary tube was partially submerged in a liquid n-pentane reservoir inside of the test chamber and the capillary height allowed for the thermocouples leads to remain outside of the liquid. Chamber vapour temperature and absolute pressure were also measured.

Moving the meniscus such that it remained at a near constant location between the tube thermocouples was accomplished via the vertical translation of the liquid reservoir inside of the test chamber. The reservoir was moved using a linear slide which was manually actuated outside of the test chamber using a rotary feedthrough. Real-time video monitoring of the meniscus was used for making fine meniscal position adjustments. A deflectometer was used to obtain the optical magnification factor and to measure the change in reservoir position throughout testing. 


\section{Chapter 4}

\section{Experimental procedure and results}

\subsection{Experimental procedure}

The following is a description of the procedure followed in order to capture meniscal instabilities inside a capillary tube.

\subsubsection{Positioning the tube}

After the tube was cleaned ultrasonically, it was mounted inside the chamber. The clamping utilised allowed for vertical and horizontal translation in addition to rotation about the vertical and horizontal translation axes. The tube was positioned inside the reservoir and close to the wall near the optical feedthrough to allow for a larger range in working distance. This was necessary since if the tube were placed at the centre of the chamber, focus would not be possible such that the meniscus would fill the computer screen during testing.

Glass beads around the tube and within the reservoir were arranged such as to create a pocket for clearance of the tube bottom. A check using the microscope was made to insure the tube was in focus and the reservoir positioning system had sufficient range to raise the reservoir during testing. The labeled thermocouple sockets were bolted to a post attached 
to the chamber baseplate. The power lead push on crimp connectors were joined to their baseplate socket.

\subsubsection{Sealing the chamber}

The chamber bell jar was crane-lifted from its position on the top shelf of the experimental test equipment shelving unit located behind the test bed. The bell jar was positioned directly over the baseplate by adjusting the crane's hydraulic ram and radial arm. The bell jar was then gently lowered using the crane winch onto three wooden spacers residing on the baseplate. The winch was then locked for added safety. The power and temperature feedthroughs were connected to their baseplate sockets. Finally, the spacers were removed and the winch lock was released for the final lowering of the chamber onto the baseplate. The bell jar was guided horizontally with one hand while controlling the vertical position via the winch with the other to insure proper docking. Once positive engagement was made between the baseplate and bell jar, lifting tension on the bell jar was applied using the winch to allow for circumferential adjustment to centre the tube with respect to the optical window. Alignment between the test tube and bell jar microscope window was made consistent by aligning vertical markings on the baseplate and bell jar.

\section{Tightening the clamps}

The 12 chamber clamps were positioned around the baseplate, each under a numbered marker on the bell jar. A torque of $20 \mathrm{Nm}$ was applied to the clamps following a bolt pattern sequence using a torque wrench. The sequence was repeated until the first bolt in the sequence was torqued to the wrench setting without additional tightening required. This tended to occur after two sequences. 


\subsubsection{Evacuating the chamber}

System evacuation was performed with the gas evacuation valve fully open, the liquid needle valve fully open, and the exterior reservoir liquid valve closed. The pump was first run on ballast mode for 5 minutes to purge solvent that may have condensed in the pump oil. The pump was then run in normal mode for about 10 minutes. This would correspond to a pressure of roughly $50 \mathrm{mTorr}$ on the log scaled gage between the pump and baseplate shutoff valve having greater accuracy at lower pressure compared to the chamber pressure gage having a linear scale from 0-1 bar. Once the chamber was evacuated, the baseplate shutoff valve was closed, the liquid needle valve was partially closed, the vacuum purge valve was opened, and the vacuum pump was shut off.

\subsubsection{Filling the reservoir}

After evacuating the chamber, $\mathrm{n}$-pentane was transferred from a $1 \mathrm{~L}$ graduated cylinder to fill the external reservoir. The exterior reservoir valve was opened resulting in evaporation of the n-pentane and drop in ambient chamber temperature. The flow of gas into the chamber was monitored visually to insure the glass beads were not disturbed and the external liquid reservoir was periodically refilled to insure it did not empty and allow air into the system. After a few minutes of gaseous n-pentane entering the chamber, the pressure reached the vapour pressure and liquid $n$-pentane began entering the chamber. The internal reservoir was filled until the meniscus resided between the thermocouples on the capillary tube. The chamber temperature dropped as a result of the expansion of n-entane vapour within the vacuum chamber and its condensation being endothermic processes. For the majority of tests, the temperature dropped below room temperature by approximately 20 ${ }^{\circ} \mathrm{C}$.

Time was allotted for the liquid to reach thermal equilibrium with the ambient test 
room. The liquid reservoir temperature was monitored via the lowest thermocouple on the capillary tube which was separated from the liquid by the tube wall glass. The screen temperature display of the DAQ was used to monitor both the chamber temperature and pressure during the air evacuation and n-pentane fill process. As the reservoir temperature increased to ambient room temperature, the meniscus level would fall as a result of the decrease in surface tension and more liquid was added by opening the external reservoir valve to bring the meniscus level back between the tube thermocouples. Final height adjustments were made using the rotary feedthrough.

\subsubsection{Data recording}

\section{Instrumentation settings}

The Keithley DAQ was programmed to record thermocouple readings converting the signals to temperature values and displaying them along with the heater and pressure transducer output voltage. Also, the resistance across the operator controlled switch to mark the presence of oscillations was recorded and displayed. The trigger delay between measurements was set to the minimum time for which the relays to switch. The measurement delay times were $1 \mathrm{~ms}$ for DC voltage and $1 \mathrm{~ms}$ for resistance [48]. Minimisation of the delay time allowed for the more accurate capture of an event in time. The timer interval between scans was chosen to be 2 seconds as this sampling rate was found to capture the physics of the system. During preliminary testing, further decrease of the scan period did not provide important additional information.

Video and audio were recorded using a second computer. The reticle transparency was placed on the computer screen and the meniscus was centred about the axis of symmetry and its height was set by having the bottom of the meniscus tangent with a horizontal marking on the screen. The meniscus was centred once before heat was applied using 
the cross slide on which the chamber resided. The position of the meniscus was adjusted throughout the test as evaporation and temperature rise would cause the meniscus to drop.

The applied voltage and current as displayed by the power supply was recorded after the voltage was raised for each power increase during testing. The change in height of the reservoir with respect to the meniscus was also recorded manually after each reservoir adjustment by noting the deflectometer reading.

\section{Testing process}

Heat was applied to the tube only after the DAQ and video began recording. Once the temperature was judged as steady by looking at the graphical temperature display, first the voltage was increased by $1 \mathrm{~V}$, then the height was adjusted until the meniscus was at its reference position. An exponential increase in temperature at the thermocouples was typically observed after a voltage step increase.

After the exponential temperature increase, one of two things would normally happen. Either the meniscus would reach a state of equilibrium (minus the evaporation), or continuous oscillations would present themselves and the meniscus was determined unstable.

After waiting and determining there was an absence of oscillatory behaviour, the reservoir was raised to place the meniscus at its reference position. The voltage was then increased by $1 \mathrm{~V}$ and the described sequence was repeated until sustained oscillatory behaviour was observed or test limits had been reached. Unsustained oscillations were observed and consisted of brief motion of the meniscus that was rapidly damped. The onset of oscillations was followed by flipping the switch marking the event in the data recording. If the oscillations were not sustained, the switch was reset. After recording the oscillatory behaviour of the meniscus for a few minutes, the power was shut off and the detection switch was reset once the oscillations subsided. The tube and reservoir were given time to 
cool until they returned to ambient room temperature and the same test was repeated twice without delay in order to show repeatability.

\section{End of test procedure}

After the three tests were completed, the DAQ and video recording were stopped simultaneously as they were started. The chamber vacuum valve and ambient purge valve were opened to bring the chamber back to ambient pressure. The liquid drain was then opened to empty the external reservoir. Afterwards, the chamber clamps were removed and the bell jar was lifted. The spacer blocks were placed between the bell jar and baseplate and the power and temperature feedthroughs were disconnected. The bell jar was then raised to its resting location on the top shelf of the racking containing the test instrumentation. The internal reservoir was drained with a piece of Tygon tubing connected to a syringe in order to create a syphon. The capillary tube was then placed aside and the reservoir was removed from the chamber and any additional n-pentane was evaporated by placing the internal reservoir on a hot plate. Leaving n-pentane inside the reservoir indefinitely was avoided as it would eventually evaporate from the reservoir and pool onto the chamber baseplate. Prolonged exposure to n-pentane would also stiffen the wiring sheathing making it more prone to cracking and exposing the bare wire. The used n-pentane was put aside separate from unused n-pentane to prevent the accumulation of contaminants.

\subsection{Data analysis}

\subsubsection{Heater power}

In order to quantify the heat input to the capillary tube, the voltage and current were recorded from the power supply after each power increment. The current reading was given 
ample time to reach steady state as the temperature increase of the heater would increase its resistance, thus decrease the current for a constant voltage source according to Ohm's law. As expected, the heater resistance increased linearly given the temperature range between ambient and around $150^{\circ} \mathrm{C}$.

\subsubsection{Steady state determination}

As heat was added to the capillary tube in a stepwise manner during the testing process, the system was given time to reach thermal equilibrium to ascertain the stability of the meniscus at that power level. Thermal equilibrium was determined by observation of the temperature readout during testing. Steady state was reached considerably faster with the thin tube due to its smaller heat capacity. In order to demonstrate the constancy of temperature throughout testing, the percent change in temperature normalised using the Kelvin temperature scale was calculated using a first-order backward difference scheme formulated in Equation (4.1) and the results are shown in Figure (4.1) and Figure (4.2) for the 1.0 $\mathrm{mm}$ ID thin tube and $1.2 \mathrm{~mm}$ ID thick tube, respectively.

$$
\left.\frac{\Delta T}{T}\right|_{i}=\frac{T\left(t_{i}\right)-T\left(t_{i-1}\right)}{T\left(t_{i}\right)}, \quad \text { where } \quad t_{i}>t_{i-1}
$$

An allowed temperature percentage change of $\Delta T / T \times 100 \%= \pm 0.01 \%$ was imposed as a limit for defining steady state and the voltage was then increased. The dips seen in the temperature change are due to raising the reservoir level which has a cooling effect on the tube wall.

Comparing Figure (4.1) with Figure (4.2), it is evident that the thin tube changes temperature much faster than the thick tube, resulting in a thin tube test time less than half as long compared to thick tube testing. The percent temperature change is larger for the thin tube due to its smaller heat capacity. The magnitude of the average scaled temperature 


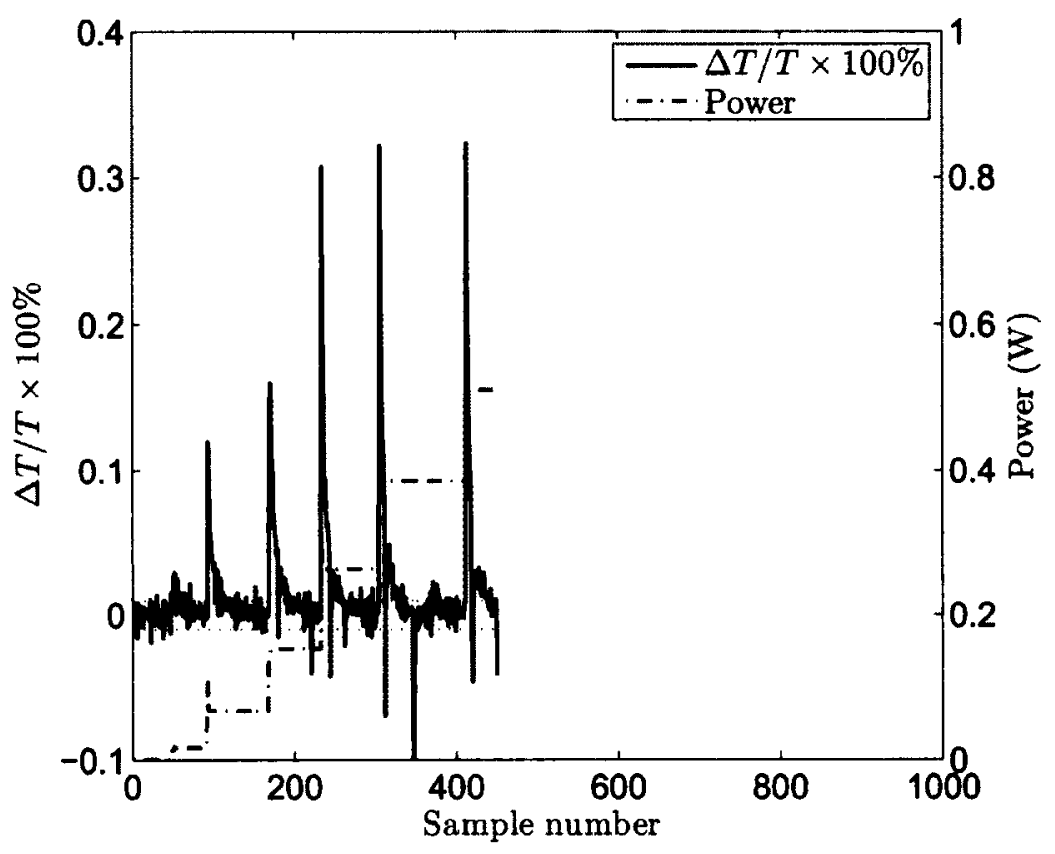

Figure (4.1): Thin tube temperature change percentage with input power and imposed limits for assumed thermal equilibrium $\pm 0.01 \%$

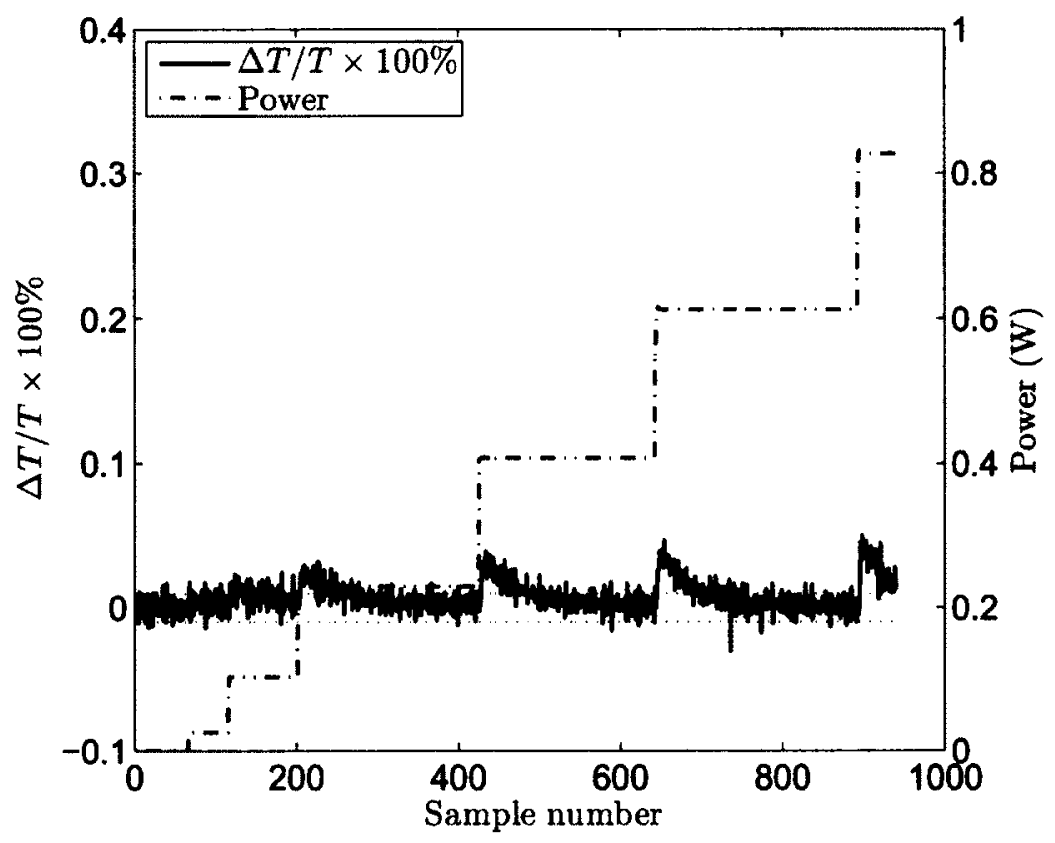

Figure (4.2): Thick tube temperature change percentage with input power and imposed limits for assumed thermal equilibrium $\pm 0.01 \%$ 
change is not plainly apparent from the figures. To show the magnitude of the average temperature change, the time derivative of the temperature change percentage can be taken considering the form in Equation (4.1), we take its numerical derivative in unit time and normalise over a test to obtain:

$$
\varphi=\frac{1}{N} \sum_{i=2}^{N}\left(\left.\frac{\Delta T}{T}\right|_{i}-\left.\frac{\Delta T}{T}\right|_{i-1}\right)^{2}
$$

where $\varphi$ is the average scaled temperature difference for a given test. Analysing the data sets shown in Figure (4.1) and Figure (4.2), it was found that $\varphi=8.4422 \times 10^{-4}$ and $\varphi=1.3059 \times 10^{-4}$ for the thin and thick tubes, respectively. This clearly shows that the temperature fluctuates more frequently in the thin tube which again makes sense due to its lower heat capacity.

\subsubsection{Meniscus imaging}

Throughout the testing process, the meniscus was observed in real time via the video feed from the microscope imager connected to the test computer. Shown in Figure (4.3) is the real time meniscus image with foreground reticle as monitored throughout testing. The meniscus height with respect to the heater level was adjusted to within \pm 1 vertical graduation on reticle. The reference point on the meniscus for this adjustment was taken as the lowest point on the liquid-vapour interface which also corresponds with the theoretical axis of symmetry. This positioning was achieved manually by observation and adjustment of the reservoir position throughout testing. The reservoir height was generally raised after a power increase since the meniscus would drop due to the increase in temperature and corresponding decrease in surface tension. The reservoir was also raised once the temperature of the meniscus stabilised after a power increase to account for evaporation. The reservoir height increase to compensate for the decrease in surface tension with temperature rise was 
greater than the reservoir height increase to compensate for evaporation. This was made evident when measuring the change capillary height after a test when the temperature had returned to ambient.

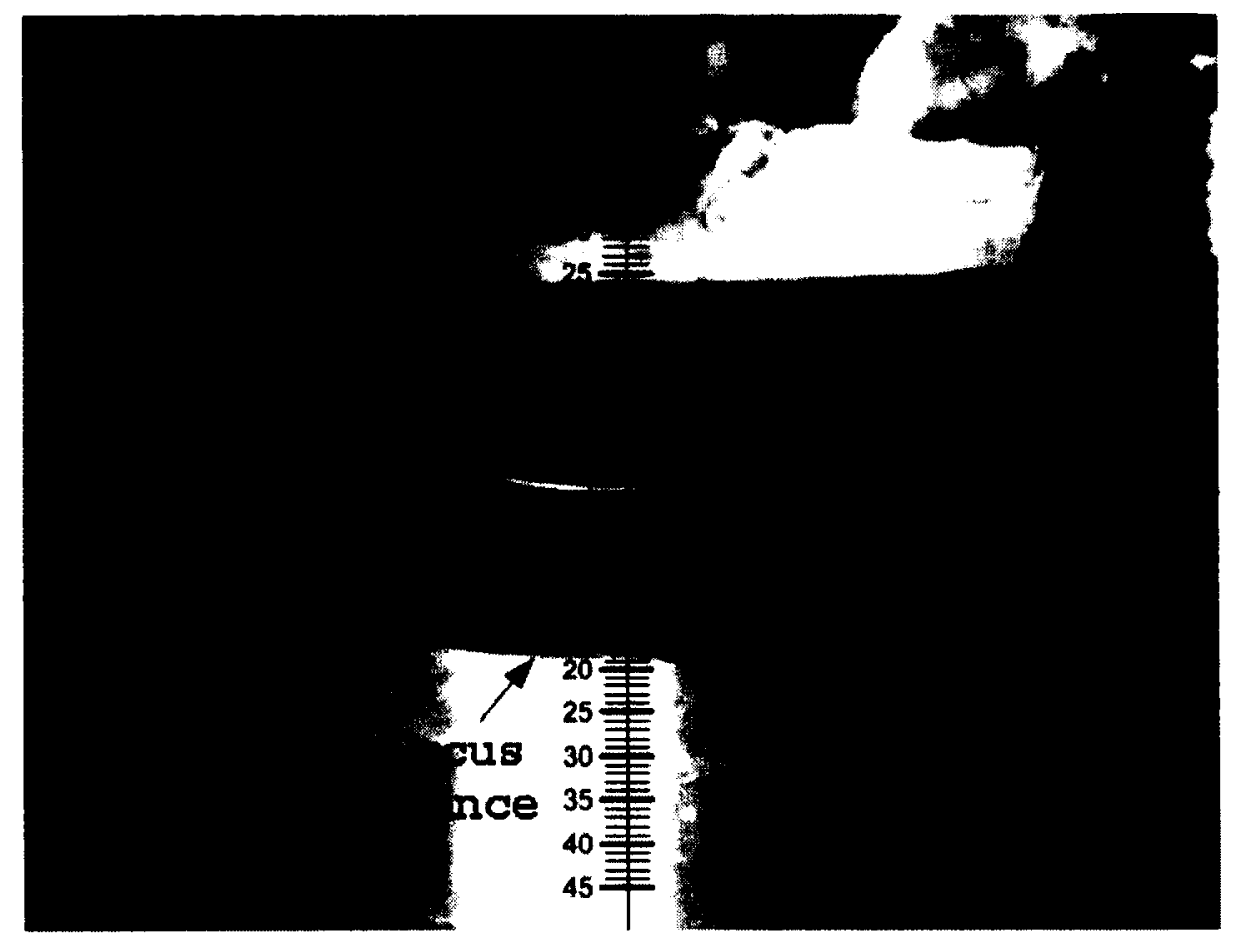

Figure (4.3): Stable meniscus as was seen during testing with foreground reticle

The magnification scale was obtained for each series of tests where the capillary tube and microscope were kept at a constant working distance and magnification. An example of the scaling data for the thin and thick tubes in vacuum is shown in Table (4.1) where the scaling factor is the size ratio between the monitor display and actual meniscus. Noting that the distance between reticle graduations is $2 \mathrm{~mm}$ we obtain scaling factors of 218 and 198 for the thin and thick tubes, respectively. The meniscus was scaled such that its width would fill the monitor width entirely. The difference in scaling factor is due to different internal diameter between tubes. 
Table (4.1): Data for obtaining magnification scaling factors for $1 \mathrm{~mm}$ ID thin, $1.2 \mathrm{~mm}$ ID thick, and $0.6 \mathrm{~mm}$ ID thick tubes in vacuum testing

\begin{tabular}{ccccccc}
\hline $\begin{array}{c}\text { tube } \\
\text { type }\end{array}$ & $\begin{array}{c}\text { ID } \\
(\mathrm{mm})\end{array}$ & $\begin{array}{c}\text { reticle } \\
\text { top }(2 \mathrm{~mm})\end{array}$ & $\begin{array}{c}\text { reticle } \\
\text { bottom }(2 \mathrm{~mm})\end{array}$ & $\begin{array}{c}\text { deflectometer } \\
\text { top }(\mathrm{mm})\end{array}$ & $\begin{array}{c}\text { deflectometer } \\
\text { bottom }(\mathrm{mm})\end{array}$ & $\begin{array}{c}\text { magnification } \\
\text { factor }\end{array}$ \\
\hline thin & 1.0 & 45 & -40 & 8.79 & 8.01 & 218 \\
thick & 1.2 & 40 & -40 & 10.67 & 9.86 & 198 \\
thick & 0.6 & 40 & -40 & 9.32 & 8.80 & 307 \\
\hline
\end{tabular}

Shown in Figure (4.4) is a sequence of three snapshots extracted every $1.5 \mathrm{~s}$ from the continuous video with oscillations present. A higher frame rate showed no change in the frequency of oscillation, thus the order of magnitude for the oscillatory period is around $3 \mathrm{~s}$.

\subsubsection{Thermocouple spacing}

The distance between thermocouples placed axially on the capillary test tubes was measured for the thick and thin tubes. Since the OD of the thick tube was greater and known with sufficient accuracy $( \pm 0.03 \mathrm{~mm})$, it was used as a reference distance for scaling with respect to the microscope eyepiece reticle. For thin tube thermocouple spacing measurement, the smaller OD was not used as a reference distance due to its smaller size and difficulty to measure. Instead the tube was fixed with respect to the linear stage via its holding bracket and the tube was moved parallel to its centre axis and the change in the deflectometer was recorded when each thermocouple aligned with respect to the same horizontal screen reticle marking. The distance between thermocouples is summarised in Table (4.2). 

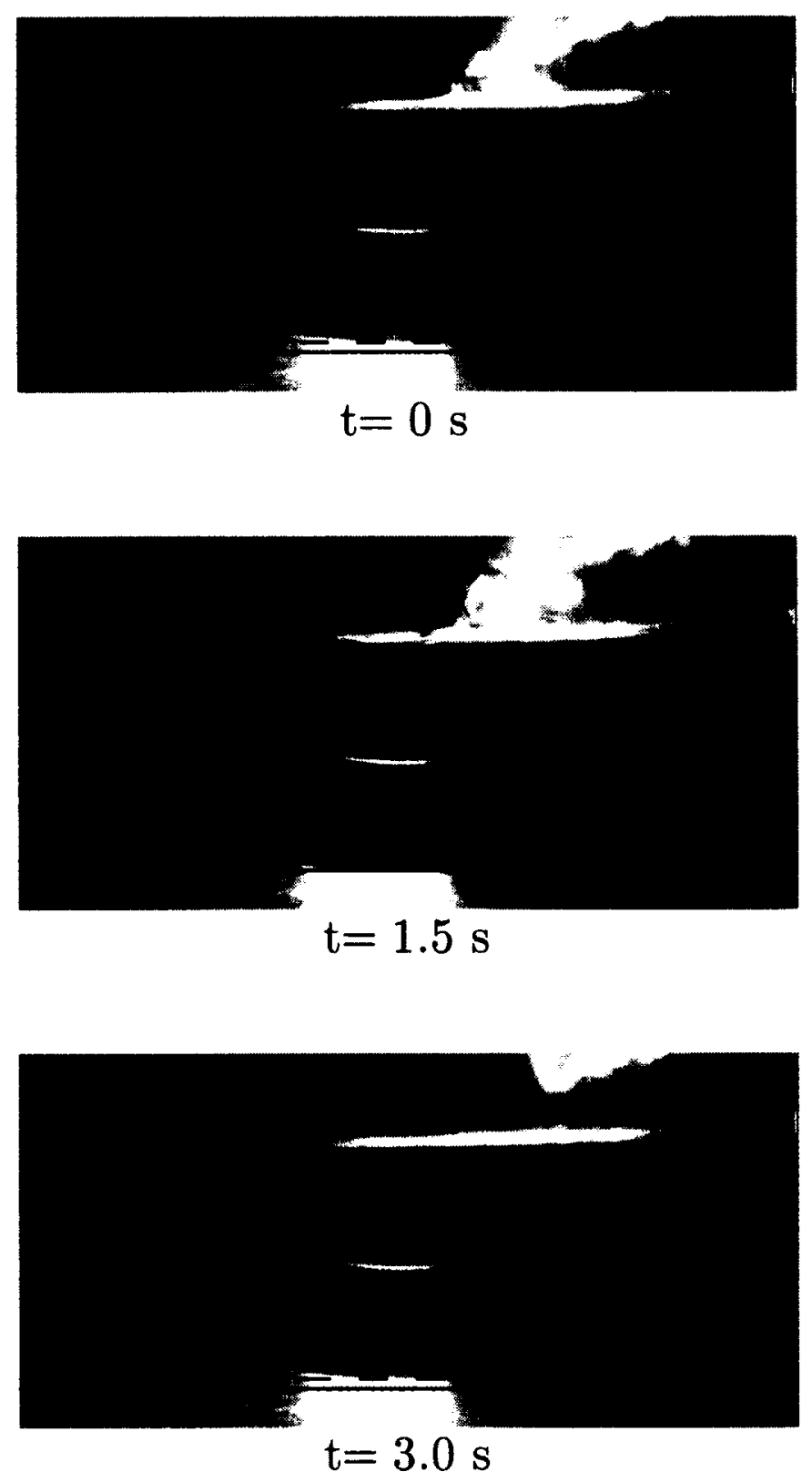

Figure (4.4): Meniscus oscillation from baseline shown at $\mathrm{t}=0 \mathrm{~s}$, dropping to a minimum at $\mathrm{t}=1.5 \mathrm{~s}$, and returning near origin at $\mathrm{t}=3.0 \mathrm{~s}$ for $1.0 \mathrm{~mm}$ inner diameter thin tube 
Table (4.2): Axial distance between thermocouples for thick and thick tubes

\begin{tabular}{cccc}
\hline \hline $\begin{array}{c}\text { tube } \\
\text { type }\end{array}$ & $\begin{array}{c}\text { tube OD } \\
(\mathrm{mm})\end{array}$ & $\begin{array}{c}\text { tube ID } \\
(\mathrm{mm})\end{array}$ & $\begin{array}{c}\text { TC spacing } \\
(\mathrm{mm})\end{array}$ \\
\hline thin & 0.7 & 0.5 & 1.56 \\
thin & 1.2 & 1.0 & 2.25 \\
thick & 5.0 & 0.6 & 3.32 \\
thick & 6.0 & 1.2 & 2.12 \\
\hline
\end{tabular}

\subsubsection{Temperature and power versus time}

In order to detect meniscal instabilities, the thermocouple wall temperature above and below the meniscus was monitored with increasing applied power. No analysis was performed to estimate the temperature at the inner wall since the temperature difference is considered more relevant. Assuming the temperature drop across the wall is approximately the same for both thermocouples, the change in temperature at the outer wall should be approximately that of the inner wall. Figure (4.5), Figure (4.6), and Figure (4.7) show the $1.0 \mathrm{~mm}$ ID thin tube in ambient, $1.0 \mathrm{~mm}$ ID thin tube in vacuum, and 1.2 ID thick tube in vacuum, respectively.

Comparing these figures, we see that the temperature difference across the meniscus is much greater for ambient testing. Given the different conditions in a multicomponent system open to ambient compared to in a closed single component environment, a difference is expected. Diffusion takes place in the ambient case which increases the complexity of analysis. Convective cooling due to air and the change of effective properties around the liquid-vapour interface have an effect that is difficult to quantify for comparison with the single component case. Models for multicomponent mass transfer about a liquid-vapour interface do exist, but require much additional work for implementation [49].

We introduce the notion that there is a difference in vapour partial pressures between the ambient and vacuum tests. We consider Dalton's partial pressure law which states 


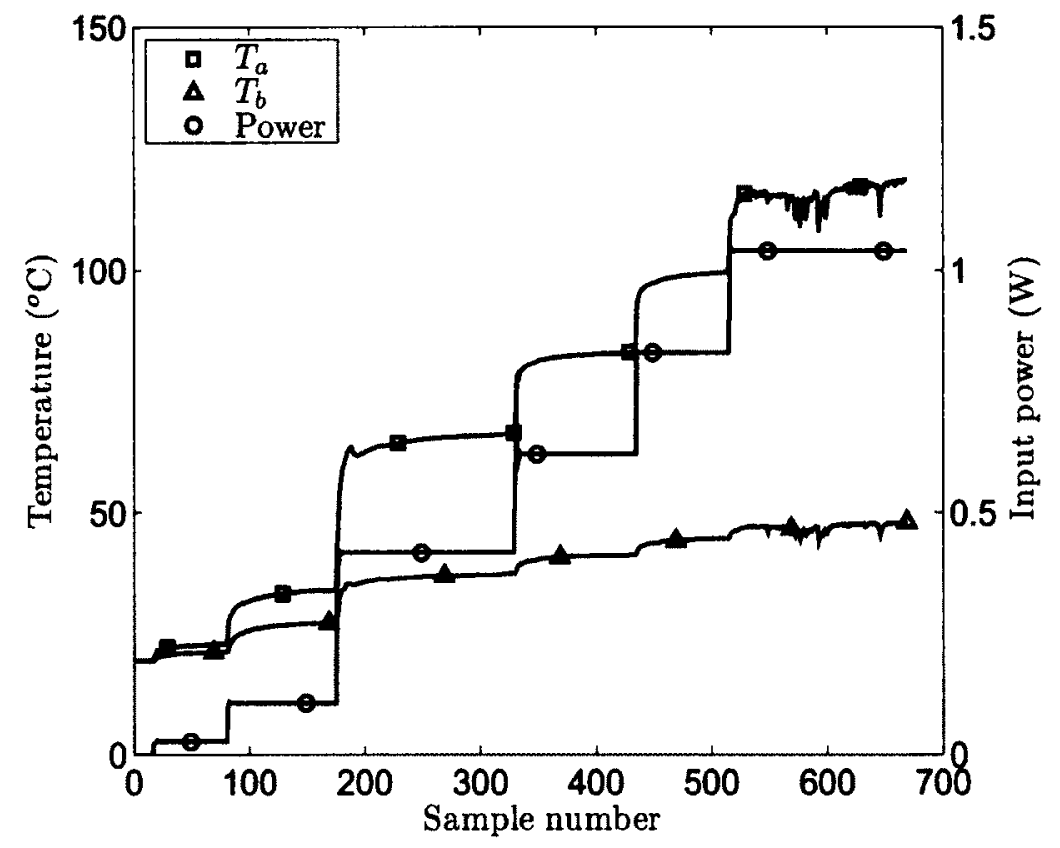

Figure (4.5): Temperature for thermocouples above and below meniscus with power input for $1.0 \mathrm{~mm}$ ID thin tube in ambient

that the pressure of a mixture comprised of non-reactive gases is equal to the sum of the hypothetical pressures of the components if each component was alone in the same volume occupied by the mixture. This is mathematically described for $N$ components comprising a mixture [50] using

$$
P_{\text {mix }}=\sum_{i=1}^{N} P_{i}
$$

The individual component pressure, $P_{i}$ can be related to the vapour pressure of a pure component, $P_{i}^{\star}$ by its mole fraction $\mathcal{X}_{i}$ in the mixture according to Raoult's law:

$$
P_{i}=\mathcal{X}_{i} P_{i}^{\star}
$$

Assuming a mixture comprised of n-pentane: (1) and air: (2), the pressure of n-pentane, $P_{1}$ 
in the mixture is thus $\mathcal{X}_{1} P_{1}^{\star}$, where $\mathcal{X}_{1}<1$, therefore the vapour pressure $P_{1}$ is less than for a single component vapour where $\mathcal{X}=1$. Unfortunately, calculating $\mathcal{X}$ is an involved task as it relates to the points mentioned above. On the positive, we are more interested in understanding single component systems from a practical standpoint since they can be used to more accurately model phase change heat transfer devices such as heat pipes.

The oscillatory behaviour once reached is more apparent for the ambient test case as there is more heat input to perpetuate the motion of the meniscus. While the meniscus is oscillating up and down, the inner wall interchangeably comes into contact with liquid and vapour. As the cooler liquid from the bulk meniscus rises above the top tube thermocouple, it carries heat away from the wall, resulting in a sudden thermocouple temperature drop. Conversely, as the bulk meniscus drops below the position of the lower tube thermocouple, substantially less heat is carried away by the resident vapour, resulting in a sudden thermocouple temperature rise. This temperature fluctuation is less seen for the thick tube as its higher thermal capacity does not allow for temperature change on a time scale coinciding with the oscillatory period of the meniscus which is in order of magnitude of approximately $1 \mathrm{~Hz}$.

We note that the oscillations observed, although maintainable, do not mean the meniscus is stable from a microscopic standpoint. Stability on the microscopic scale would not result in the macroscopic oscillations measured by the thermocouples. The macroscopic oscillatory motion implies microscopic instability since the macroscopic contains the microscopic. The smallest oscillation one could detect with this setup while still viewing the entire meniscus would be the smallest detectable movement on the camera display divided by the magnification factor. Assuming a bias uncertainty for reading the screen reticle, $B_{\mathrm{SR}}=2 \mathrm{~mm}$, then the smallest oscillation possible would be $B_{\mathrm{SR}} / \mathrm{MF}$. This would result approximately $0.01 \mathrm{~mm}$ and $0.007 \mathrm{~mm}$ as detectable oscillatory amplitudes for the larger and smaller ID capillaries, respectively. One could in theory zoom into the meniscus and 


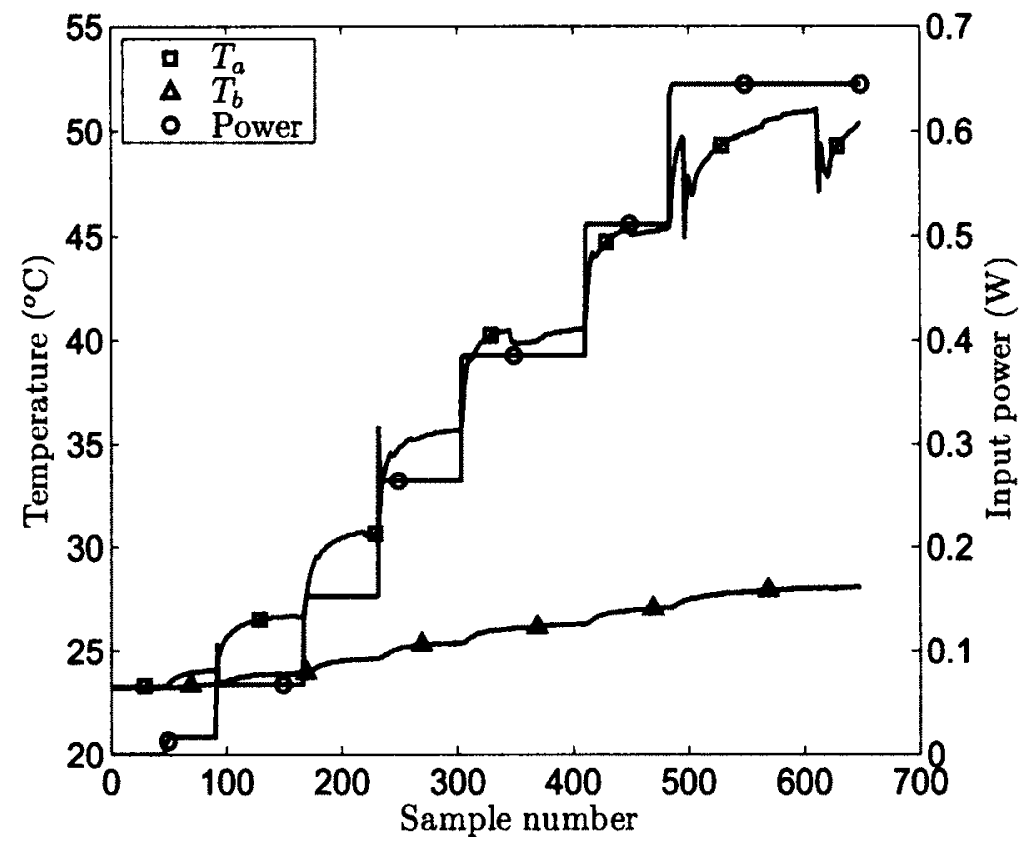

Figure (4.6): Temperature for thermocouples above and below meniscus with power input for $1.0 \mathrm{~mm}$ ID thin tube in vacuum

focus on a smaller portion with more magnification, but this would be at the expense of controlling its position. Alternatively, a finer meniscus position control mechanism could be developed in order to allow for observation at higher magnification. However, the observation magnification used in this study was satisfactory since the more easily observable macroscopic effects are of practical importance.

We now show the results from tube testing for the $0.6 \mathrm{~mm}$ ID thick tube and $0.5 \mathrm{~mm}$ ID thin tube. The thermocouple wall temperatures with increasing input power are shown for the thick and thin tubes in Figure (4.8) and Figure (4.9), respectively.

Although not evident by observing the temperature profile of the top thermocouple for the thick tube, the meniscus did oscillate at the highest power setting shown in the Figure (4.8). For the thin tube, the top thermocouple temperature did vary at lower power settings, but the variation was not sustained, indicating stability as the meniscus itself appeared stationary through the microscope. The last power setting shows the divergence of 


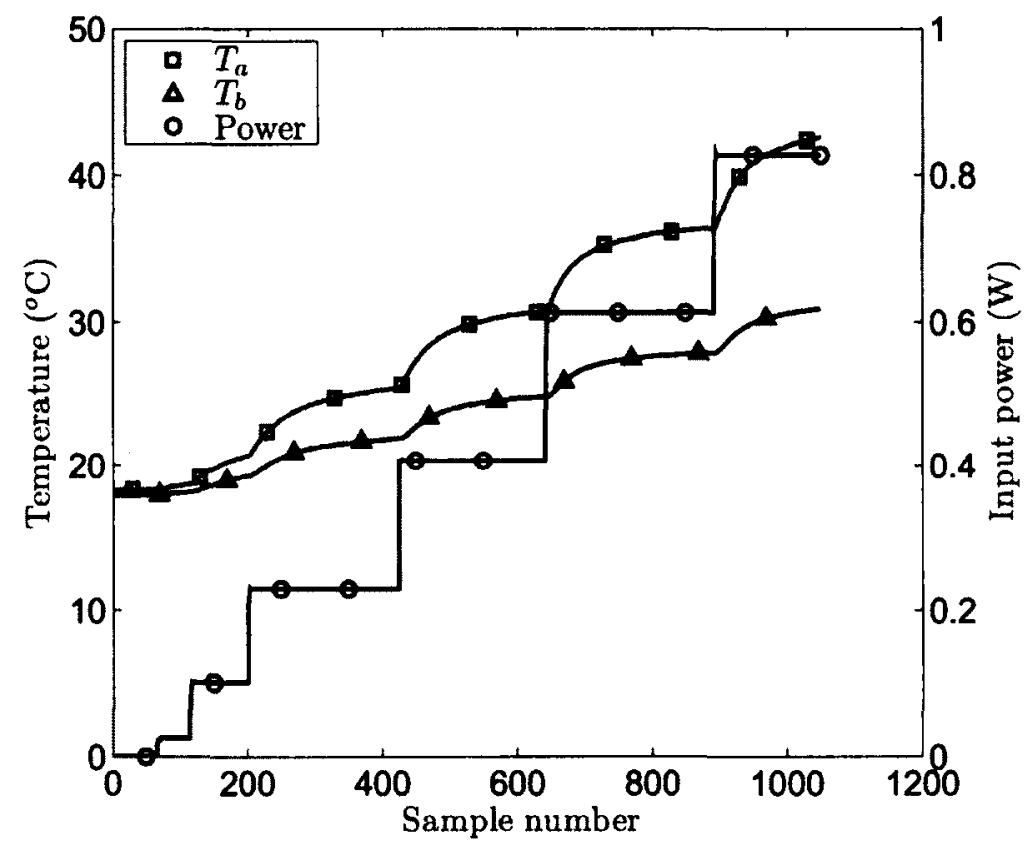

Figure (4.7): Temperature for thermocouples above and below meniscus with power input for $1.2 \mathrm{~mm}$ inner diameter thick tube in vacuum

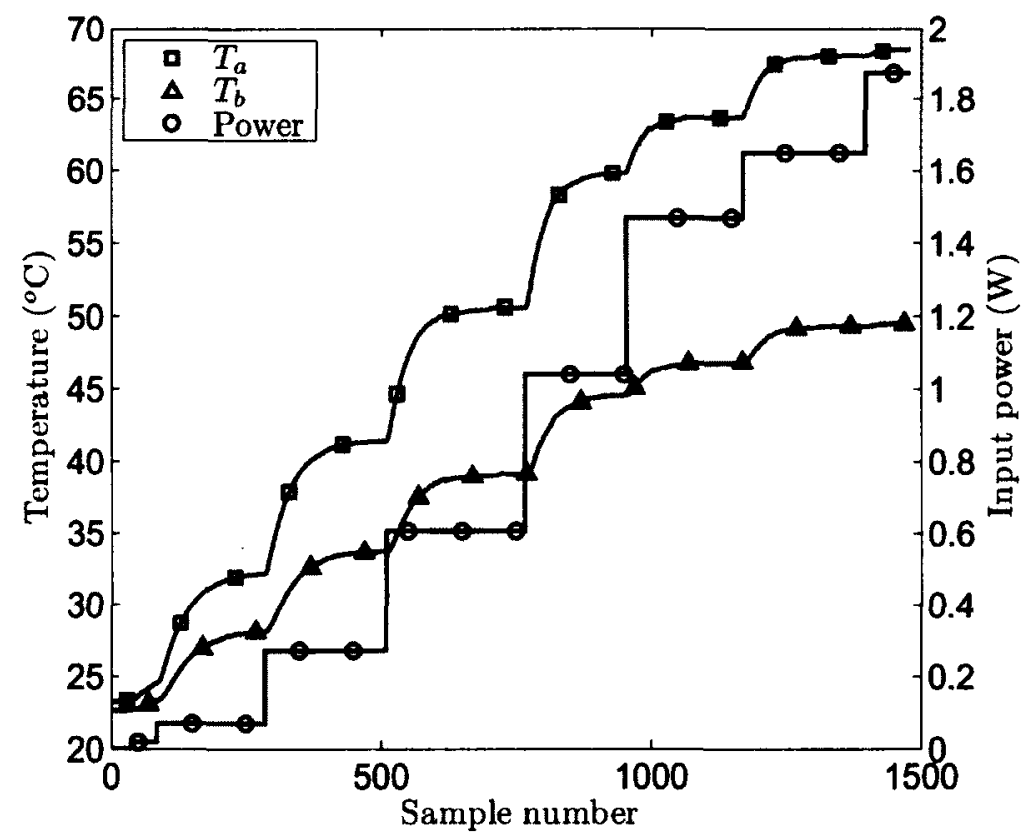

Figure (4.8): Temperature for thermocouples above and below meniscus with power input for $0.6 \mathrm{~mm}$ ID thick tube in vacuum 


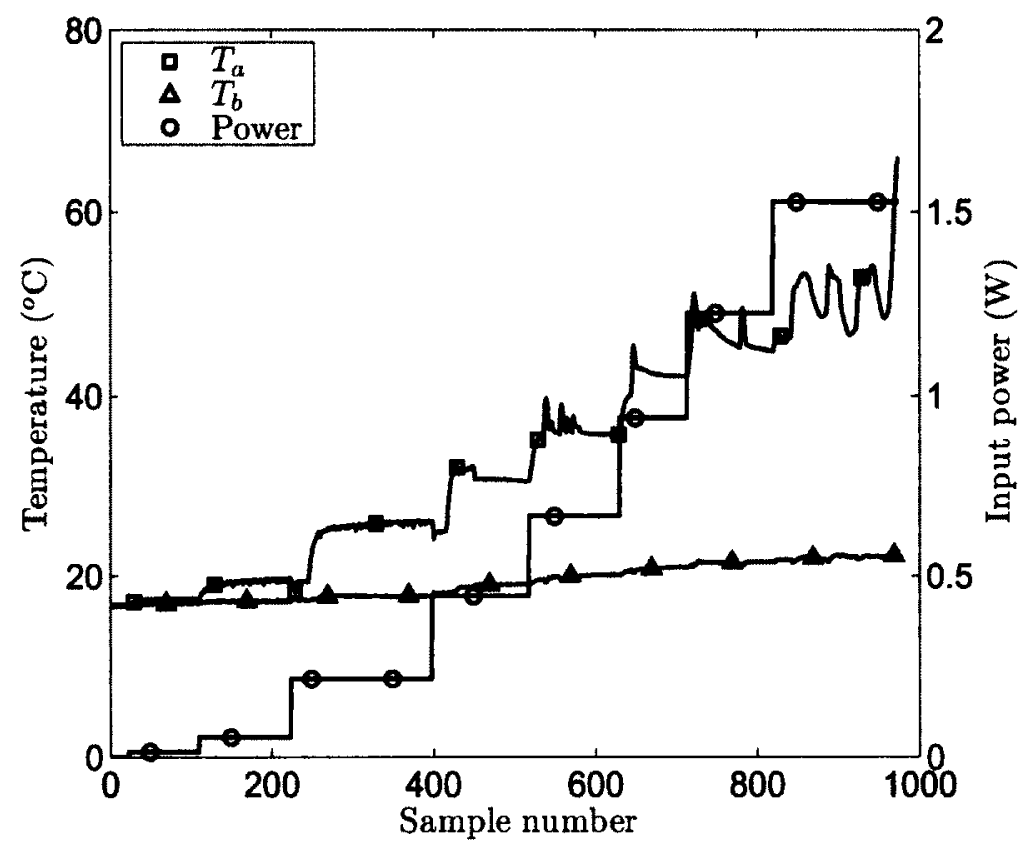

Figure (4.9): Temperature for thermocouples above and below meniscus with power input for $0.5 \mathrm{~mm}$ ID thin tube in vacuum

the temperature which corresponds to tube burnout. The variation of temperature prior to burnout is noted to be a consequence of adjusting the meniscus position as it readily moved away from its reference position. Thus, the $0.5 \mathrm{~mm}$ ID thin tube is not considered unstable by the mode which is typified by an oscillating meniscus at around $1 \mathrm{~Hz}$.

The slower moving away of the meniscus could be considered as a form of instability, but a higher sampling rate of tracking and adjusting the capillary height with respect to the reservoir would have to be included to accurately judge this behaviour. Also, the theory developed in the following chapter does not consider the entirety of the liquid within a capillary tube above a reservoir. Therefore, the position of the meniscus with respect to the heat source requires constancy to match the later proposed theory which focuses on the thin film.

Shown in Table (4.3) is a summary of temperatures above and below the meniscus, their difference, and the linear temperature gradient across the meniscus at the onset of 
Table (4.3): Meniscus thermocouple temperatures and gradient at onset of instability. ${ }^{*}$ Meniscus still considered stable

\begin{tabular}{cccccc}
\hline $\begin{array}{c}\text { tube } \\
\text { type }\end{array}$ & $\begin{array}{c}\text { tube ID } \\
(\mathrm{mm})\end{array}$ & $\begin{array}{c}\bar{T}_{a} \\
\left({ }^{\circ} \mathrm{C}\right)\end{array}$ & $\begin{array}{c}T_{b} \\
\left({ }^{\circ} \mathrm{C}\right)\end{array}$ & $\begin{array}{c}\Delta T_{a b} \\
\left({ }^{\circ} \mathrm{C}\right)\end{array}$ & $\begin{array}{c}\Delta T_{a b} / L \\
\left({ }^{\circ} \mathrm{C} / \mathrm{mm}\right)\end{array}$ \\
\hline thin & 0.5 & 65.9 & 22.9 & 43.0 & $27.5^{*}$ \\
thin & 1.0 & 50.0 & 27.1 & 22.9 & 10.2 \\
thick & 0.6 & 68.5 & 49.5 & 19.0 & 5.71 \\
thick & 1.2 & 38.1 & 28.4 & 9.70 & 4.58 \\
\hline
\end{tabular}

instability for all cases except for the case of the $0.5 \mathrm{~mm}$ ID thin tube where maximum heat applied before heater burnout is shown. All tests were conducted three times (except for the burnout case) and results for the other two cases are not shown nor is an average taken since they were close within experimental uncertainty.

A larger temperature difference is sensed for the thin tubes and correspondingly a larger temperature gradient. The temperature difference is approximately inversely proportional to the tube internal diameter for both thin and thick tube cases.

\subsubsection{Comparing the measured temperature gradient for thick and thin tubes}

A larger temperature gradient is observed for the thin tubes compared to the thick tubes at the onset of instability. This is due to the higher axial thermal conduction for the thick tube compared to the thin tube. Given that the temperature gradient of interest is across the liquid-vapour interface, the temperature difference between thermocouples in thick tube testing is considered less representative seeing as they are further from the liquid-vapour interface than for thin tube testing. 


\subsubsection{Average temperature difference variation with applied power}

The average temperature was determined by taking the arithmetic mean of the last 40 temperature data samples before changing the power setting. Shown in Figure (4.10) is the average temperature difference between thermocouples across the meniscus at steady state versus applied power for ambient and vacuum testing. The results shown are up to the power setting before macroscopic oscillations arose.

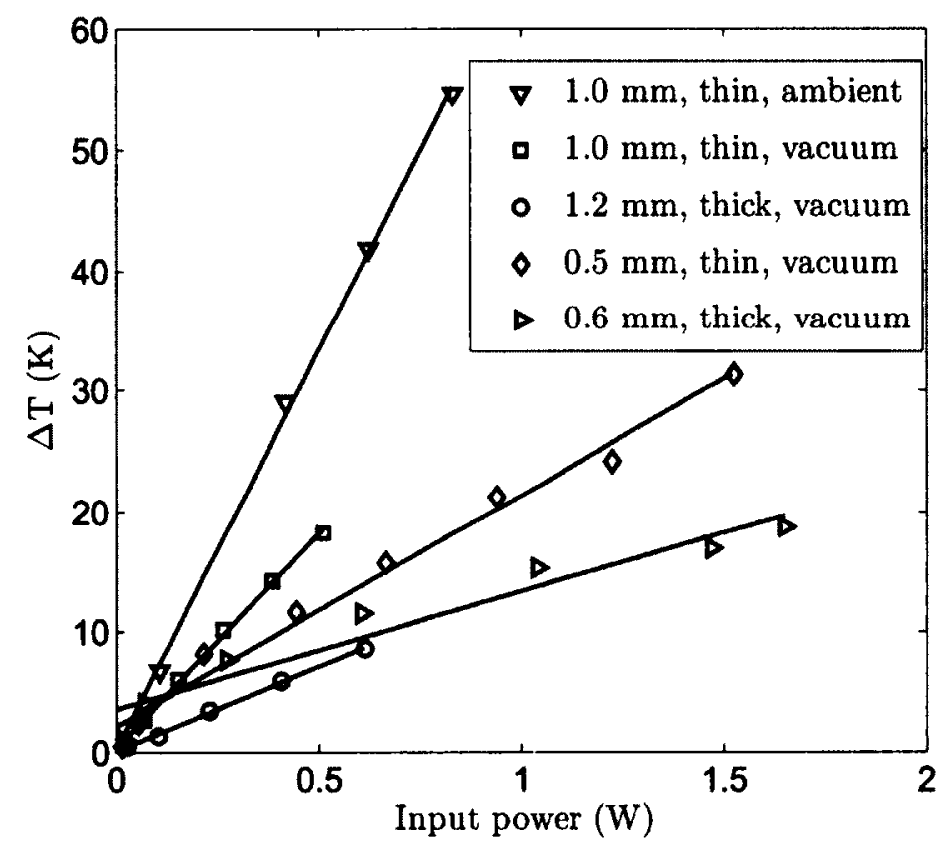

Figure (4.10): Thermocouple average temperature change versus applied power for thick and thin tubes for vacuum and ambient testing

The results yield an approximately linear relationship between applied power and temperature difference. The slope of the lines in Figure (4.10) can be used to compare the resistance to heat transferred along the tubes with a larger slope indicating a greater resistance. We define the absolute thermal resistance $R_{\theta}$ according to Equation (4.5):

$$
R_{\theta}=\frac{\Delta T}{Q}
$$


The ambient case has the highest resistance and this is attributed to the differences in the evaporation process in an air-pentane mixture compared to a pure n-pentane environment. Although the thermal resistance was greater, more net heat was transferred via the ambient versus vacuum test case since more heat was required to dryout the meniscus.

Clearly, the thick tubes have a lower resistance compared to the thin tubes due to having a larger cross-sectional area. When comparing between IDs of the same class of tube (thin or thick), the smaller inner diameter tubes have a lower resistance. Since the smaller ID tubes also have a smaller corresponding OD, convection losses are less due to a smaller surface area.

\subsubsection{Uncertainty analysis}

The total uncertainty on a derived quantity, $f=f\left(x_{1}, x_{2}, \ldots, x_{n}\right)$ can be calculated based on its bias uncertainty, $B_{u}$ and its precision uncertainty, $S_{u}$ [51]. The bias uncertainty denotes how close the measurement is to the true value and can be known based on the calibration of the sensor. Precision uncertainty refers how repeatable is a measurement and the error magnitude increases with the randomness of the test parameter. In this analysis, we consider a $95 \%$ confidence interval for the precision uncertainty which is typical in engineering uncertainty analysis [52]. The precision uncertainty claimed is that the parameter in question will be within the uncertainty given, for $95 \%$ of all test points.

The total uncertainty can be calculated via a root sum squared (RSS) approach which combines the bias and precision uncertainty according to

$$
\mathcal{U}_{95}=\sqrt{B_{u}^{2}+S_{u}^{2}}
$$

The bias uncertainty is obtained by an RSS combination of all bias uncertainty terms involved with the measurement chain used to obtain the parameter as shown in 


$$
B_{u}=\sqrt{\sum_{i=1}^{N}\left(f_{x_{i}} B_{i}\right)^{2}}
$$

where the index $i$ refers to the individual component within the measurement chain containing $N$ elements. We note that the individual components in the chain do not necessarily have the same units as the measured quantity, thus we include the sensitivity parameter, $f_{x_{i}}$ which is the partial derivative of the derived quantity with respect to the independent variable, $x_{i}$. The precision uncertainty, $S_{u}$ can be obtained by using Student's $t$ distribution and takes the form of

$$
S_{u}=\frac{t_{S} \sigma_{s t}}{\sqrt{N}}
$$

where $N$ is the number of measurements and $t_{S}$ is the inverse of Student's $t$ cumulative distribution function which is dependent on the number of measurements (degrees of freedom), $N$, and the chosen confidence interval of $95 \%$ for a two-sided interval. The numerical value for $t_{S}$ is obtained using the built in MATLAB function 'tinv', which is based on a one-sided interval, thus $97.5 \%$ is used in the function which is equivalent to a $95 \%$ two-sided interval. The standard deviation, $\sigma_{s t}$ is obtained for the $N$ measurements via

$$
\sigma_{s t}=\sqrt{\frac{1}{N} \sum_{i=1}^{N}\left(x_{i}-\bar{f}\right)^{2}}
$$

where $\bar{f}$ is the mean measurement value calculated using

$$
\bar{f}=\frac{1}{N} \sum_{i=1}^{N} f_{i}
$$


Table (4.4): Coefficients for DAQ polynomial conversion of voltage to temperature

\begin{tabular}{|c|c|}
\hline$c_{1}$ & $2.592800 \mathrm{e}-2$ \\
$c_{2}$ & $-7.602961 \mathrm{e}-7$ \\
$c_{3}$ & $4.637791 \mathrm{e}-11$ \\
$c_{4}$ & $-2.165394 \mathrm{e}-15$ \\
$c_{5}$ & $6.048144 \mathrm{e}-20$ \\
$c_{6}$ & $-7.293422 \mathrm{e}-25$ \\
\hline
\end{tabular}

\section{Temperature uncertainty}

We now look at the error associated with the temperature measurement which is arguably the most significant measurement in this study. The T-type thermocouples were used as stock with $0.5{ }^{\circ} \mathrm{C}$ special limit of error as quoted by the supplier. The accuracy of the thermocouple wire is taken as the special limit of error.

The uncertainty associated with the cold junction compensation of the Keithley 7708 multiplexer card with a mainframe temperature between $18-28{ }^{\circ} \mathrm{C}$ results in a total uncertainty of $\pm 1.0^{\circ} \mathrm{C}$.

The ITS-90 (International temperature scale of 1990) conversion of thermocouple voltage to temperature between $0-400{ }^{\circ} \mathrm{C}$ is achieved via the polynomial fit in Equation (4.11) where the voltage is in $\mu \mathrm{V}$ and temperature is in degrees Celsius. Coefficients corresponding to Equation (4.11) are given in Table (4.4) result in an uncertainty of $\pm 0.03^{\circ} \mathrm{C}$.

$$
T=\sum_{i=1}^{N} c_{i} V^{i}
$$

The uncertainty on the voltage measurement of the Keithley DAQ is $30 \mathrm{ppm}$ of the read voltage plus $35 \mathrm{ppm}$ of the voltage range. Since all thermocouple voltages in this study are on the order of less than $10 \mathrm{mV}$, the measurement for all readings falls within the DAQ range of 0-100 mV. Assuming a maximum voltage of $6.704 \mathrm{mV}$ which corresponds to approximately $150^{\circ} \mathrm{C}$, the associated voltage measurement bias uncertainty is $3.701 \mu \mathrm{V}$. 
The sensitivity, $T_{V}$ can be calculated by taking the derivative of Equation (4.11) and solving at the highest voltage of $6.704 \mathrm{mV}$ via

$$
\begin{aligned}
T_{V} & =\left.\sum_{i=1}^{N} i c_{i} V^{i-1}\right|_{V=6.704 \mathrm{mV}} \\
& =0.02^{\circ} \mathrm{C} / \mu \mathrm{V}
\end{aligned}
$$

We now have all the individual component bias uncertainties and can proceed to find the bias uncertainty for the temperature measured by a thermocouple:

$$
B_{u}=\sqrt{B_{\mathrm{SL}}^{2}+B_{\mathrm{CJC}}^{2}+2 B_{\mathrm{VT}}^{2}+\left(T_{V} B_{\mathrm{V}}\right)^{2}}
$$

where $B_{\mathrm{SL}}, B_{\mathrm{CJC}}, B_{\mathrm{VT}}$, and $B_{\mathrm{V}}$ are the uncertainties for the special limit of error thermocouple wire, cold junction compensation, voltage to temperature conversion, and voltage, respectively. We note that the voltage to temperature conversion uncertainty is included twice because voltage is converted to temperature twice to obtain the temperature, rather than simply a temperature difference. One conversion accounts for the voltage difference between the thermocouple lead and cold junction compensation, and the second conversion accounts for the voltage difference between the cold junction compensation and ground. Using Equation (4.13), we calculate the bias uncertainty on one thermocouple temperature measurement to be $\pm 1.1^{\circ} \mathrm{C}$. When finding the uncertainty due to the temperature difference, we account for the uncertainty of both measurements to obtain:

$$
B_{u_{\Delta T}}=\sqrt{2} B_{u}
$$


which yields a bias uncertainty of $\pm 1.6^{\circ} \mathrm{C}$.

Now, we can consider the precision uncertainty, $S_{u}$ which is relevant when measuring steady state temperature before increasing the heat input to a stable meniscus. For the thin $1.0 \mathrm{~mm}$ ID tube, we consider the last 25 top and bottom thermocouple temperature samples before power increase. We calculate temperature difference as it is more relevant than temperature alone in our analysis. The average standard deviation of the temperature difference at steady state for all heat inputs was calculated as $\sigma_{s t}=0.05^{\circ} \mathrm{C}$. We obtain a t-statistic of $t_{S}=2.056$ which results in a precision uncertainty of $S_{u}= \pm 0.019^{\circ} \mathrm{C}$ using Equation (4.8). Taking the RSS value of bias and precision errors according to Equation (4.6) gives a total error on the temperature difference between thermocouples to be $U_{\Delta T_{\mathcal{T}}}=$ $\pm 1.6^{\circ} \mathrm{C}$.

In a similar fashion, we obtain the standard deviation and precision uncertainty of the thick $1.2 \mathrm{~mm}$ ID tube to be $\sigma_{s t}=0.06^{\circ} \mathrm{C}$ and $S_{u}= \pm 0.023^{\circ} \mathrm{C}$, respectively. The precision uncertainty is higher compared to the thin tube because the temperature was not as steady given the larger heat capacitance of the thick tube meant significantly longer time would be required to attain a comparable stability. The greater precision error introduced by not waiting longer for the transient to subside was insignificant in comparison to the bias error.

\section{Thermocouple spacing uncertainty}

The distance between thermocouples and the capillary height change have their associated bias uncertainties. The error in the distance between thermocouples depends on the reference dimension bias uncertainty, $B_{\text {act }}$ of the tube outer diameter which was measured using callipers with a known bias uncertainty of $\pm 0.02 \mathrm{~mm}$ for the thick tubes. We assume the ocular reticle to have negligible bias error compared to the callipers, but assume a bias error, $B_{\text {ret }}=0.5$ of a graduation in reading the ocular reticle being half its resolution. For the thick tube, we formulate the distance between thermocouples, $L$ based on the ratio of the 
reticle spacing between the thermocouples, $L_{\text {ret }}$ and tube OD, $d_{\text {ret }}$ with respect to the known actual tube $\mathrm{OD}, d_{\text {act }}$ according to

$$
L=\frac{L_{\mathrm{ret}}}{d_{\mathrm{ret}}} d_{\mathrm{act}}
$$

By using the form of the thermocouple spacing in Equation (4.15), we use Equation (4.7) to calculate the associated bias uncertainty on the distance between thermocouples for the $1.2 \mathrm{~mm}$ ID thick tube:

$$
\begin{aligned}
B_{L} & = \pm \sqrt{2\left[\left(L_{L_{\mathrm{ret}}} B_{\mathrm{ret}}\right)^{2}+\left(L_{d_{\mathrm{ret}}} B_{\mathrm{ret}}\right)^{2}+\left(L_{d_{\mathrm{act}}} B_{\mathrm{act}}\right)^{2}\right]} \\
& = \pm \sqrt{2\left[\left(\frac{d_{\mathrm{act}}}{d_{\mathrm{ret}}} B_{\mathrm{ret}}\right)^{2}+\left(\frac{-L_{\mathrm{ret}} d_{\mathrm{act}}}{d_{\mathrm{ret}}^{2}} B_{\mathrm{ret}}\right)^{2}+\left(\frac{L_{\mathrm{ret}}}{d_{\mathrm{ret}}} B_{\mathrm{act}}\right)^{2}\right]} \\
& = \pm \sqrt{2\left[\left(\frac{4.91 \mathrm{~mm}}{110} 0.5\right)^{2}+\left(\frac{-182 \cdot 4.91 \mathrm{~mm}}{110^{2}} 0.5\right)^{2}+\left(\frac{182}{110} 0.02 \mathrm{~mm}\right)^{2}\right]} \\
& = \pm 0.08 \mathrm{~mm}
\end{aligned}
$$

We note that the terms appear twice in the radical because two measurements are made to obtain a difference. A different approach is taken to calculate the thermocouple spacing bias uncertainty for the thin tubes. An uncertainty of $\pm 10 \%$ of the tube ID was given by the manufacturer for the thin tubes and corresponds approximate uncertainty of $\pm 0.1 \mathrm{~mm}$ for the $1 \mathrm{~mm}$ ID tube if wall thickness error is neglected. Thin tube OD was not measured using callipers due to tube fragility. Since the bias uncertainty quoted by the manufacturer is significantly greater than that for the calliper measurement uncertainty of the thick tubes, the deflectometer with a bias uncertainty, $B_{\text {def }}=0.03 \mathrm{~mm}$ was used to measure the travel of 
the axially moving capillary tube as the thermocouples crossed a horizontal ocular reticle marking fixed with respect to the microscope. We assume a cumulative alignment and reading error of $B_{\mathrm{al}}=0.02 \mathrm{~mm}$ between the thermocouple centre and ocular reticle marking. As such, the bias uncertainty for the thin tube thermocouple spacing can be calculated via

$$
\begin{aligned}
B_{L} & = \pm \sqrt{2 U_{\mathrm{def}}^{2}+2 U_{\mathrm{al}}^{2}} \\
& = \pm \sqrt{2(0.03 \mathrm{~mm})^{2}+2(0.02 \mathrm{~mm})^{2}} \\
& = \pm 0.05 \mathrm{~mm}
\end{aligned}
$$

\section{Capillary height change uncertainty}

The uncertainty of the capillary height change is dependent on the deflectometer accuracy in a manner similar to the thin tube thermocouple spacing bias uncertainty. The main difference compared to the thermocouple spacing measurement is the alignment error which results from reading the screen reticle marking versus the ocular reticle marking for the thermocouple spacing. To illustrate, we consider the magnification factor, $M F=218$ for the $1.0 \mathrm{~mm}$ ID thin tube and we assign an error in positioning the meniscus as one graduation on the screen reticle (SR), $B_{\mathrm{SR}}=2 \mathrm{~mm}$.

The bias uncertainty for the change in capillary height, $\Delta h$ can thus be calculated via Equation (4.18a). We note that the bias for the deflectometer, $B_{\text {def }}$ and screen reticle bias, $B_{\mathrm{SR}}$ appear twice to obtain a change. The bias error of the magnification factor, $B_{\mathrm{MF}}$ is neglected.

$$
B_{\Delta h}= \pm \sqrt{2\left[B_{\mathrm{def}}^{2}+\left(\frac{1}{\mathrm{MF}} B_{\mathrm{SR}}\right)^{2}+\left(\frac{\Delta \mathrm{f}_{\mathrm{SR}} B_{\mathrm{MF}}}{\mathrm{MF}^{2}}\right)^{2}\right]}
$$




$$
\begin{aligned}
& = \pm \sqrt{2\left[(0.03 \mathrm{~mm})^{2}+\left(\frac{2}{218} \mathrm{~mm}\right)^{2}\right]} \\
& = \pm 0.04 \mathrm{~mm}
\end{aligned}
$$

Although not performed in this study, one could calculate the precision uncertainty of the meniscus position by tracking its motion via the video recording. An edge detection algorithm could be used to provide a large number of samples and reduce the overall precision uncertainty as described by Equation (4.8).

\section{Temperature gradient uncertainty}

Now that both uncertainties for temperature difference and thermocouple spacing are known, we can calculate the uncertainty bias on the temperature gradient.

For the $1.0 \mathrm{~mm}$ ID thin tube with a thermocouple temperature difference, $\Delta T=20^{\circ} \mathrm{C}$, the error on the temperature gradient is obtained via

$$
\begin{aligned}
U_{\Delta T / \mathrm{L}} & = \pm \sqrt{\left(\frac{1}{\mathrm{~L}} U_{\Delta T \tau}\right)^{2}+\left(\frac{\Delta T}{\mathrm{~L}^{2}} B_{\mathrm{L}}\right)^{2}} \\
& = \pm \sqrt{\left(\frac{1}{2.25 \mathrm{~mm}} 1.6^{\circ} \mathrm{C}\right)^{2}+\left(\frac{20^{\circ} \mathrm{C}}{(2.25 \mathrm{~mm})^{2}} 0.05 \mathrm{~mm}\right)^{2}} \\
& = \pm 0.8^{\circ} \mathrm{C} / \mathrm{mm}
\end{aligned}
$$

Similarly for the $1.2 \mathrm{~mm}$ ID thick tube with a thermocouple temperature difference, 
$\Delta T=10^{\circ} \mathrm{C}$, the error on the temperature gradient is obtained via

$$
\begin{aligned}
U_{\Delta T / \mathrm{L}} & = \pm \sqrt{\left(\frac{1}{\mathrm{~L}} U_{\Delta T_{\tau}}\right)^{2}+\left(\frac{\Delta T}{\mathrm{~L}^{2}} B_{\mathrm{L}}\right)^{2}} \\
& = \pm \sqrt{\left(\frac{1}{2.12 \mathrm{~mm}} 1.6^{\circ} \mathrm{C}\right)^{2}+\left(\frac{10^{\circ} \mathrm{C}}{(2.12 \mathrm{~mm})^{2}} 0.08 \mathrm{~mm}\right)^{2}} \\
& = \pm 0.8^{\circ} \mathrm{C} / \mathrm{mm}
\end{aligned}
$$

\section{Vapour chamber pressure uncertainty}

We now look at the uncertainty of the vapour pressure measurement within the chamber via the pressure transducer. A linear calibration curve was provided to convert transducer output voltage to absolute pressure. This curve can be used to find the change in pressure with respect to voltage, $P_{V}$ which is required in the uncertainty calculation. The manufacturer provided a maximum total bias uncertainty on the pressure of $B_{\mathrm{BSL}}=0.08 \% \mathrm{BSL}$ (best straight line) around room temperature. The maximum vapour pressure sensed by the transducer during the series of three tests for the $1 \mathrm{~mm}$ ID thin tube was, $P_{v_{\max }}=9.27$ psia which results in $B_{\mathrm{BSL}}=7.41 \times 10^{-3}$ psia. We note that the calibration was given to 0.01 psia and thus add an additional uncertainty to account for the calibration uncertainty of $B_{\mathrm{CAL}}=0.005$ psia. The voltage output between $0-5 \mathrm{~V}$ of the transducer corresponded to a reading uncertainty of $B_{\mathrm{V}}= \pm(30 \mathrm{ppm}$ of reading $+5 \mathrm{ppm}$ of range $)$ by the DAQ [53]. The maximum reading for pressure encountered was on the order of $3 \mathrm{~V}$ which falls in the range of 1-10 V. We use this information to calculate the bias uncertainty on the vapour 
pressure via

$$
\begin{aligned}
B_{\mathrm{P}} & = \pm \sqrt{\left(B_{\mathrm{BSL}}\right)^{2}+\left(B_{\mathrm{CAL}}\right)^{2}+\left(P_{\mathrm{V}} B_{\mathrm{V}}\right)^{2}} \\
& = \pm \sqrt{\left(7.41 \times 10^{-3} \mathrm{psia}\right)^{2}+(0.005 \mathrm{psia})^{2}+\left(3.00 \mathrm{psia} / \mathrm{V} \cdot 1.4 \times 10^{-4} \mathrm{~V}\right)^{2}} \\
& = \pm 8.95 \times 10^{-3} \mathrm{psia} \\
& = \pm 61.7 \mathrm{~Pa}
\end{aligned}
$$

Now the precision error can be calculated in the same way as the steady state temperature error described previously. We take all, $N=2641$ measurements for the three tests in series to obtain a standard deviation, $\sigma_{s t}=195 \mathrm{~Pa}$ and a precision uncertainty, $S_{u}=7.46 \mathrm{~Pa}$. This results in a total uncertainty $U_{\mathcal{T}}=62.2 \mathrm{~Pa}$. The low standard deviation of the vapour pressure for the duration of three serial tests validates the constant pressure approximation.

\section{Power input uncertainty}

The power input, $\mathcal{P}$ was subject to a bias uncertainty, $B_{\mathcal{P}}$ based on the accuracy of the power supply which was quoted to display the true voltage and current to within $0.5 \%$ of the reading and at most 2 digits off from the last significant digit on the screen display. For the highest voltage and current values before dryout for the $1 \mathrm{~mm}$ ID thin tube, this results in $B_{V}= \pm\left(0.05 V_{\max }+0.02 \mathrm{~V}\right)$ and $B_{I}= \pm\left(0.05 A_{\max }+0.002 \mathrm{~A}\right)$. We can now obtain the power input measurement bias according to

$$
\begin{aligned}
B_{\mathcal{P}} & = \pm \sqrt{\left(\mathcal{P}_{V} B_{\mathrm{V}}\right)^{2}+\left(\mathcal{P}_{I} B_{\mathrm{I}}\right)^{2}} \\
& = \pm \sqrt{\left(I B_{\mathrm{V}}\right)^{2}+\left(V B_{\mathrm{I}}\right)^{2}}
\end{aligned}
$$




$$
\begin{aligned}
& = \pm \sqrt{(0.092 \mathrm{~A} \cdot 0.055 \mathrm{~V})^{2}+(7.00 \mathrm{~V} \cdot 0.00246 \mathrm{~A})^{2}} \\
& = \pm 0.0179 \mathrm{~W}
\end{aligned}
$$

A precision uncertainty in the power measurement does play a role in the analysis since the resistance of the heating element increases with temperature, thus decreasing the power input to the tube under constant voltage operation. This being the case, the increase in element temperature will cause a decrease in heat input and thus result in a negative feedback system which will tend to self-stabilise.

We note that the heat input to the tube is not critical in the context of this study since the applied heat is used to as a means to impose a temperature gradient which is accounted for more thoroughly via measurement. The stability theory developed in the next chapter does not depend on input power, making it of secondary importance relative to temperature. For future theoretical development which is more dependent on input power, the use of a more stringently self-regulating power supply is suggested. A feedback mechanism which senses the current draw and adapts accordingly to output a near constant power is recommended. Also, a programmed step input of predetermined power level could make for easier comparability between tests and eliminate the need for power adjustment by the test operator. 


\section{Chapter 5}

\section{Stability theory}

We now develop a theory which describes a thin film and its evolution in time when its height is perturbed. A criterion which determines the minimum superheat to destabilise the thin film will be derived based on the thin film physics and scaling of the governing equations and boundary conditions. The film itself is assumed to be two-dimensional, continuous, and monotonically increasing in height. It is bound by its vapour on one side and a heated, solid, planar, non-reactive surface on the other.

\subsection{Geometry and coordinate system}

Physically, the film can be used to approximate half of a meniscal cross section divided along its plane of symmetry. In order to determine whether the film is stable or not, we apply a perturbation to the base state in the form of a wave which disrupts the film surface. The base film is compared to its perturbed version as shown in Figure (5.1). The evolution of the perturbed liquid-vapour interface in time is examined as the effects of surface tension and its gradient due to temperature variation, disjoining pressure, and phase change interact to determine the film profile and its stability when perturbed. A two-dimensional Cartesian 


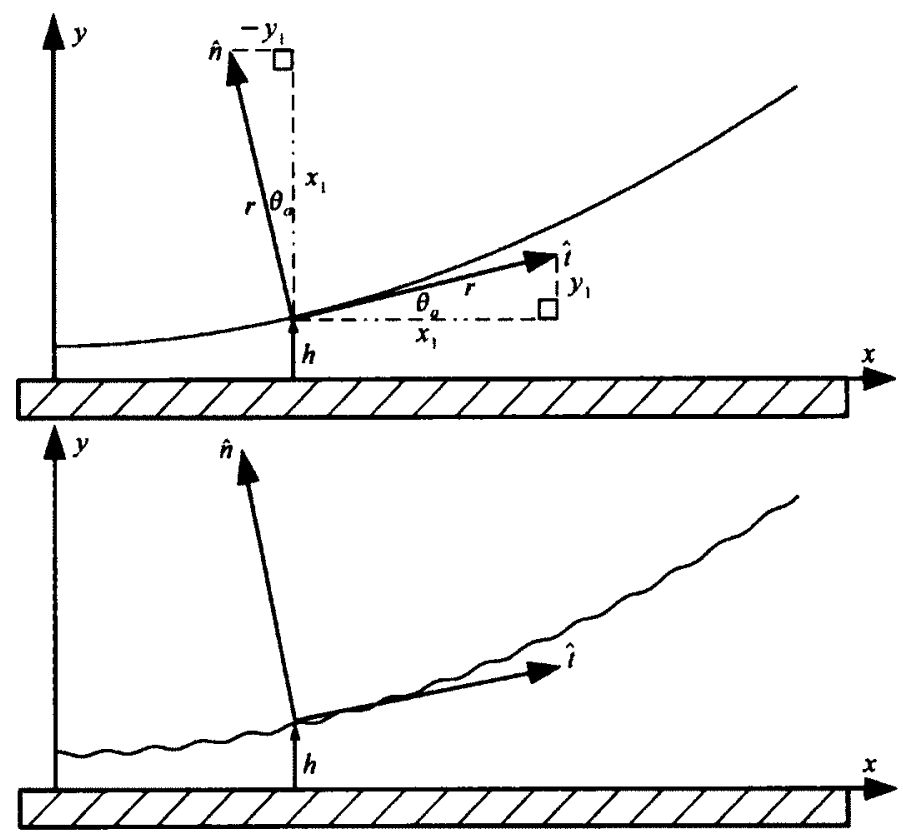

Figure (5.1): Geometry of a curved thin film evaporating on heated surface, Top: base state and geometry for unit vectors. Bottom: perturbed state

coordinate system is used with axes parallel and normal to the solid surface. Normal and tangential unit vectors with respect to the film liquid-vapour interface at height $h$ are derived based on the geometry in Figure (5.1). The right triangle with a geometry dependent on the curvature at the interface is expressed as

$$
r^{2}=x_{1}^{2}+y_{1}^{2}
$$

Taking the point of origin of $x_{1}$ and $y_{1}$ as the intersection of the two mutually perpendicular unit vectors, shown in Figure (5.1), we obtain Equation (5.2) and Equation (5.3)

$$
\begin{gathered}
\hat{t}=\frac{\left(x_{1}, y_{1}\right)}{r} \\
\hat{n}=\frac{\left(-y_{1}, x_{1}\right)}{r}
\end{gathered}
$$


Given the slope of the liquid-vapour interface, $h_{x}=y_{1} / x_{1}$, we divide Equation (5.2) and Equation (5.3) by $x_{1}$ and obtain:

$$
\begin{gathered}
\hat{t}=\frac{\left(1, h_{x}\right)}{r / x_{1}} \\
\hat{n}=\frac{\left(-h_{x}, 1\right)}{r / x_{1}}
\end{gathered}
$$

From Equation (5.1), we can obtain the magnitude of $r / x_{1}$ in terms of the slope of the interface as

$$
\frac{r}{x_{1}}=\sqrt{1+h_{x}^{2}}
$$

Finally, substituting Equation (5.6) into Equation (5.2) and Equation (5.3) yields the tangential and normal unit vectors as

$$
\begin{aligned}
& \hat{t}=\left(1, h_{x}\right)\left(1+h_{x}^{2}\right)^{-\frac{1}{2}} \\
& \hat{n}=\left(-h_{x}, 1\right)\left(1+h_{x}^{2}\right)^{-\frac{1}{2}}
\end{aligned}
$$

\subsection{Governing equations and boundary conditions}

We are now ready to describe the equations which govern the physics within the thin film and the boundary conditions at the wall and liquid-vapour interface. The equations within the film are described in the $x$ (parallel) and $y$ (normal) directions with respect to the flat substrate. We consider mass conservation, momentum conservation parallel and normal to the substrate, and energy conservation. 
The interface conditions are referred to as 'jump' conditions since a discontinuity is apparent in pressure due to capillary and disjoining effects, velocity due to density change with evaporation, and energy due to the latent heat of vaporisation. Mass flux is assessed normal to the interface since we assume the net evaporative direction is normal to the interface. Capillary pressure is assessed normal to the interface and gradients in surface tension are assessed tangentially to the interface.

\subsubsection{Mass conservation}

First, we describe mass conservation within the film for a two-dimensional domain as

$$
\rho_{t}+(\rho u)_{x}+(\rho v)_{y}=0
$$

where $\rho, u, v$ represent the density, speed parallel, and speed normal to the wall, respectively. Treating the liquid as incompressible permits one to write Equation (5.9) as

$$
u_{x}+v_{y}=0
$$

\subsubsection{Momentum conservation}

Conservation of momentum in the $x$-direction is written for an incompressible Newtonian fluid as the 2D Navier-Stokes equation:

$$
\rho\left(u_{t}+u u_{x}+v u_{y}\right)=-P_{x}+\mu\left(u_{x x}+u_{y y}\right)
$$

where $P$ and $\mu$ represent the pressure and dynamic viscosity, respectively. Similarly, momentum within the film in the $y$-direction is described by the 2D Navier-Stokes equation: 


$$
\rho\left(v_{t}+u v_{x}+v v_{y}\right)=-P_{y}+\mu\left(v_{x x}+v_{y y}\right)
$$

We neglect gravitational effects since the length scale of the thin film to be modelled in the following chapter is small $\mathcal{O}\left(L_{c}\right) \sim \mathrm{nm}$, making the Bond number, $B o=\rho_{l} g L_{c}^{2} / \sigma \ll 1$ for n-pentane and most common fluids under the influence of earth's gravitational field. Disjoining pressure has the effect of a body force on the film, but will be modelled as a pressure jump across the liquid vapour interface.

\subsubsection{Energy conservation}

The energy equation within the film at temperature, $T$, assuming constant and isotropic thermal conductivity, $k$ for an incompressible fluid without viscous dissipation, is

$$
\rho c_{p}\left(T_{t}+u T_{x}+v T_{y}\right)=k\left(T_{x x}+T_{y y}\right)
$$

\subsubsection{Boundary conditions}

\section{Wall conditions}

We now focus on the boundary conditions surrounding the thin film. At the wall $(y=0)$, we assign a constant temperature $T_{w}$, no slip between the wall and liquid, and no suction or injection of material normal to the wall. Collectively, these yield the following boundary conditions:

$$
\begin{gathered}
\left.T\right|_{y=0}=T_{w} \\
\left.u\right|_{y=0}=0
\end{gathered}
$$




$$
\left.v\right|_{y=0}=0
$$

\section{Mass flux}

Turning our attention now to the liquid-vapour interface, we first consider the mass flux as liquid evaporates. Mass flux across the interface is described using

$$
J=\rho \vec{V} \cdot \hat{n}
$$

where $\vec{V}(x, y)$ is the velocity of the fluid at the interface relative to the moving interface and $\hat{n}$ is the unit vector normal to the interface. This relative velocity is significant because it is used to quantify the extent by which molecules are evaporating. The height of the film, $h$, changes with time as evaporation occurs and, as such, the velocity of the liquid at the interface has a relative component in the $y$-direction. Since evaporation is assumed to occur normal to the interface, the interface will also move in the $x$ direction and so liquid moving in the $x$-direction will have a corresponding relative velocity component as

$$
\vec{V}=\left(u+\tan \left(\theta_{a}\right) h_{t}, v-h_{t}\right)
$$

where speeds $u$ is parallel to and speed $v$ is normal to the wall. Physically, the term $\tan \left(\theta_{a}\right)$ is typically small for a thin film and we therefore neglect the relative velocity term to simplify Equation (5.16) in favour of Equation (5.17), or

$$
\vec{V}=\left(u, v-h_{t}\right)
$$


Substituting this velocity expression and the expression for the normal unit vector from Equation (5.8), into the mass flux expression, Equation (5.15), and performing the dot product operation gives

$$
\begin{aligned}
& J=\rho\left(u, v-h_{t}\right) \cdot\left(-h_{x}, 1\right)\left(1+h_{x}^{2}\right)^{-\frac{1}{2}} \\
& J=\rho\left(-h_{x} u+v-h_{t}\right)\left(1+h_{x}^{2}\right)^{-\frac{1}{2}}
\end{aligned}
$$

\section{Normal stress balance}

Momentum across the interface is balanced by the disjoining pressure, capillary pressure, and viscous stress. We first discuss the effects of surface tension, $\sigma$, which is frequently modelled as being linearly dependent on temperature such that:

$$
\sigma(T)=\sigma_{o}\left(T_{\text {ref }}\right)-\gamma\left(T-T_{\text {ref }}\right)
$$

where the reference temperature, $T_{\text {ref, }}$, is taken as the saturation temperature for $\sigma_{o}$, and it is considered equal to the vapour temperature. The symbol, $\gamma=-d \sigma / d T$ for the rate of change of surface tension with temperature is positive for the fluid (n-pentane) in this study. The momentum balance across the interface in the normal direction reduces to the expression:

$$
P-P_{v}+\sigma \kappa+|\Pi|-\left[\left(\mathcal{T}-\mathcal{T}_{v}\right) \cdot \hat{n}\right]^{\top} \cdot \hat{n}=\frac{J_{v}^{2}}{\rho_{v}}-\frac{J^{2}}{\rho}
$$

where ${ }^{\top}$ is the transpose operator and the non-subscripted terms correspond to the liquid phase. The density and viscosity differences across the interface are large such that $\mu \gg \mu_{v}$ and $\rho \gg \rho_{v}$, and, as such, we can neglect the vapour stress, $\mathcal{T}_{v}$ and $J^{2} / \rho_{l}$, respectively in 
Equation (5.20). Vapour pressure in the bulk, $P_{v}$, is assumed constant. We note that the physics of the system are such that capillary pressure, $\sigma \kappa$, and the absolute value of the disjoining pressure, $|\Pi|$, both act in the same sense to create a pressure difference between the liquid and vapour; hence their signs are the same. The absolute value bars are shown to remove ambiguity from the inconsistent sign convention in the literature. From hereon in this derivation, the disjoining pressure will be assumed positive. This is significant since the Hamaker constant calculated in Equation (2.7b) is negative.

Expressing the curvature, $\kappa$ as a function of geometry using Equation (2.10) and neglecting the terms mentioned above yields

$$
P-P_{v}+\sigma h_{x x}\left(1+h_{x}^{2}\right)^{-\frac{3}{2}}+|\Pi|-(\mathcal{T} \cdot \hat{n})^{\top} \cdot \hat{n}=\frac{J^{2}}{\rho_{v}}
$$

We now look at the stress tensor, $\mathcal{T}$, and how it can be expressed. The viscous stress due to fluid motion has various components shown in Figure (5.2). Equation (5.22) gives a general formulation for describing the stress for a two-dimensional, incompressible $(\nabla \cdot \vec{V}=0)$, Newtonian fluid element [54], where $V_{j}$ is the general velocity, $X_{j}$ is the general direction, and the index $j$ refers to a specific direction. Thus we can write the stress tensor as

$$
\mathcal{T}_{i j}=\mu\left(V_{i, j}+V_{j, i}\right)
$$

Writing Equation (5.22) in matrix form for the two-dimensional element in Figure (5.2) yields:

$$
\mathcal{T}=\mu\left[\begin{array}{cc}
2 u_{x} & u_{y}+v_{x} \\
v_{x}+u_{y} & 2 v_{y}
\end{array}\right]
$$

The viscous stress term in Equation (5.21) can be written using the stress tensor, $\mathcal{T}$, in 


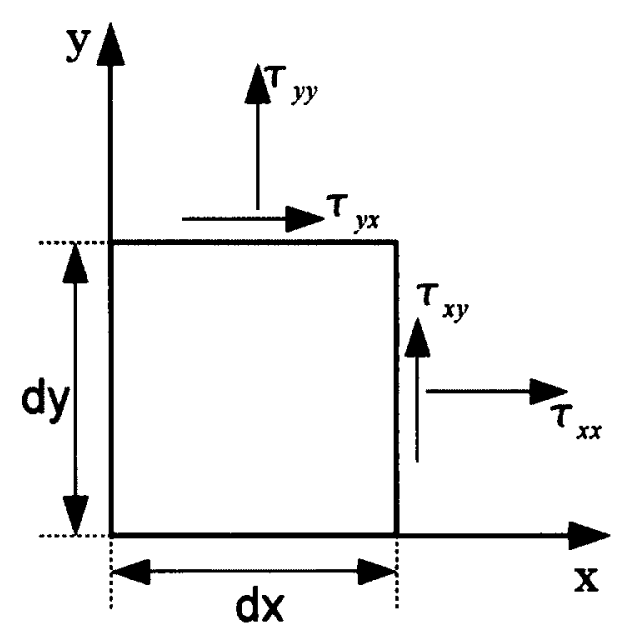

Figure (5.2): Viscous stress acting on a two-dimensional fluid element

Equation (5.23) and mass conservation in Equation (5.10) for an incompressible fluid in two dimensions to give Equation (5.24f) where the substitution in Equation (5.24e) uses the mass conservation expression given in Equation (5.10).

$$
\begin{aligned}
(\mathcal{T} \cdot \hat{n})^{\top} \cdot \hat{n} & =\mu\left(1+h_{x}^{2}\right)^{-1}\left(\left[\begin{array}{cc}
2 u_{x} & u_{y}+v_{x} \\
v_{x}+u_{y} & 2 v_{y}
\end{array}\right]^{-}\left[\begin{array}{c}
-h_{x} \\
1
\end{array}\right]\right)^{\top}\left[\begin{array}{c}
-h_{x} \\
1
\end{array}\right] \\
& =\mu\left(1+h_{x}^{2}\right)^{-1}\left[\begin{array}{r}
-2 h_{x} u_{x}+u_{y}+v_{x} \\
-h_{x}\left(v_{x}+u_{y}\right)+2 v_{y}
\end{array}\right]^{\top}\left[\begin{array}{c}
-h_{x} \\
1
\end{array}\right] \\
& =\mu\left(1+h_{x}^{2}\right)^{-1}\left[-2 h_{x} u_{x}+u_{y}+v_{x}-h_{x}\left(v_{x}+u_{y}\right)+2 v_{y}\right]\left[\begin{array}{c}
-h_{x} \\
1
\end{array}\right] \\
& =\mu\left(1+h_{x}^{2}\right)^{-1}\left[2 h_{x}^{2} u_{x}-h_{x}\left(v_{x}+u_{y}\right)-h_{x}\left(v_{x}+u_{y}\right)+2 v_{y}\right] \\
& =\mu\left(1+h_{x}^{2}\right)^{-1}\left[2 h_{x}^{2} u_{x}-h_{x}\left(v_{x}+u_{y}\right)-h_{x}\left(v_{x}+u_{y}\right)-2 u_{x}\right] \\
& =2 \mu\left(1+h_{x}^{2}\right)^{-1}\left[u_{x}\left(h_{x}^{2}-1\right)-h_{x}\left(v_{x}+u_{y}\right)\right]
\end{aligned}
$$


Substituting the viscous stress described by Equation $(5.24 \mathrm{f})$, the cubic formulation for the disjoining pressure in Equation (2.3), and the temperature dependent surface tension in Equation (5.19) into of Equation (5.21) gives

$$
\begin{aligned}
P-P_{v}+h_{x x}\left[\sigma_{o}-\gamma(T-\right. & \left.\left.T_{\mathrm{ref}}\right)\right]\left(1+h_{x}{ }^{2}\right)^{-\frac{3}{2}}+\frac{\bar{A}}{h^{3}} \ldots \\
& -2 \mu\left[u_{x}\left(h_{x}^{2}-1\right)-h_{x}\left(v_{x}+u_{y}\right)\right]\left(1+h_{x}^{2}\right)^{-1}=\frac{J^{2}}{\rho_{v}}
\end{aligned}
$$

\section{Tangential stress balance}

Balancing momentum in the tangential direction involves the interaction between the viscous stress and surface tension gradient, but without flux components since there is no net evaporation assumed tangential to the interface. This assumption is based on the approximation of tangential evaporation being isotropic. Interfacial shear results from the relative liquid motion and the surface tension gradient results from temperature variation along the interface. Similar to Equation (5.25), one can write the tangential stress balance by taking the tangential stress component of Equation (5.23) and balancing it with the gradient of Equation (5.19) and using continuity in Equation (5.10) to obtain:

$$
\begin{aligned}
-\left[\left(\mathcal{T}-\mathcal{T}_{v}\right) \cdot \hat{n}\right]^{\top} \cdot \hat{t}+\nabla \sigma \cdot \hat{t} & =0 \\
{[\mathcal{T} \cdot \hat{n}]^{\top} \cdot \hat{t} } & =\nabla \sigma \cdot \hat{t}
\end{aligned}
$$




$$
\begin{aligned}
{\left[-2 h_{x} u_{x}+u_{y}+v_{x}\right.} & \left.-h_{x}\left(v_{x}+u_{y}\right)+2 v_{y}\right]\left[\begin{array}{c}
1 \\
h_{x}
\end{array}\right]=-\left(1+h_{x}^{2}\right)^{\frac{1}{2}} \gamma\left[\begin{array}{ll}
T_{x} & T_{y}
\end{array}\right]\left[\begin{array}{c}
1 \\
h_{x}
\end{array}\right] \\
{\left[-2 h_{x} u_{x}+u_{y}+v_{x}-h_{x}^{2}\left(v_{x}+u_{y}\right)+2 h_{x} v_{y}\right] } & =-\left(1+h_{x}^{2}\right)^{\frac{1}{2}} \gamma\left(T_{x}+T_{y} h_{x}\right) \\
{\left[-2 h_{x} u_{x}+u_{y}+v_{x}-h_{x}^{2}\left(v_{x}+u_{y}\right)-2 h_{x} u_{x}\right] } & =-\left(1+h_{x}^{2}\right)^{\frac{1}{2}} \gamma\left(T_{x}+T_{y} h_{x}\right) \\
\mu\left(u_{y}+v_{x}\right)\left(1-h_{x}^{2}\right)-4 \mu u_{x} h_{x} & =-\gamma\left(T_{x}+T_{y} h_{x}\right)\left(1+h_{x}^{2}\right)^{\frac{1}{2}}
\end{aligned}
$$

where the one-sided model neglects the term accounting for the viscous stress of vapour in Equation (5.26a) and mass continuity is used to go from Equation (5.26d) to Equation (5.26e).

\subsubsection{Energy jump}

Evaporation is associated with an energy jump since the sensible heat from conduction within the film is converted to the latent heat of the vapour leaving the interface. With no work done, neglecting viscous energy dissipation, and the component of the velocity in the $x$ direction normal to the interface; the energy jump across the interface:

$$
J\left\{\mathcal{L}+\frac{1}{2}\left[\left(v_{v}-h_{t}\right) \cdot \hat{n}\right]^{2}-\frac{1}{2}\left[\left(v-h_{t}\right) \cdot \hat{n}\right]^{2}\right\}=k_{v} \nabla T \cdot \hat{n}-k \nabla T \cdot \hat{n}
$$

where the left hand side represents the latent heat, $\mathcal{L}$, and the kinetic energy difference. The right hand side is the conductive heat transfer difference. Noting that $k_{v} \ll k_{l}$, we neglect the vapour conductivity term. By also neglecting the kinetic energy in Equation (5.27), we 
obtain

$$
\begin{aligned}
J \mathcal{L} & =-k \nabla T \cdot \hat{n} \\
& =k\left(T_{x} h_{x}-T_{y}\right)\left(1+h_{x}^{2}\right)^{-\frac{1}{2}}
\end{aligned}
$$

\subsubsection{Constitutive equation for resistance to evaporation}

We can describe evaporation using the definition of interfacial thermal resistance in Equation (2.15) and the kinetic theory approximation for the resistance given in Equation (2.17) to obtain an expression for evaporative mass flux, $J$, according to

$$
J=\frac{\alpha \rho_{v} \mathcal{L}}{T_{v}^{3 / 2}}\left(\frac{M}{2 \pi R_{u}}\right)^{1 / 2}\left(T_{l v}-T_{v}\right)
$$

where $\alpha$ is the accommodation coefficient, $R_{u}$ is the universal gas constant, and $M$ is the molecular mass of the fluid.

\subsection{Scaling the governing equations and boundary condi- tions}

\subsubsection{Scaling parameters}

The motivation for non-dimensionalisation is to find characteristic lengths in $x$ and $y$ which may be relevant to the physics for a curved, evaporating interface. These lengths may be used to predict the destabilising conditions for an evaporating thin film. We define $x_{c}$ and $h_{c}$ as the respective horizontal and vertical characteristic lengths. 
This study proposes the characteristic length, $h_{c}$, to be the film height where maximum evaporation occurs. This location is considered representative of where Marangoni convection at the interface is the largest. After the film is perturbed, the interfacial trough corresponding to the maximum mass flux will experience a pull from either side of the interface due to the greater surface tension of the cooler interface above the trough in the crest. The pull in turn convects liquid away from the trough. As liquid is pulled away from the trough, the height of the thin film is further decreased, resulting in greater evaporation due to the smaller distance required for heat to travel from the superheated liquid to the interface. This is a runaway effect that makes this location the most likely place where dryout will occur for a given superheat.

We now propose the characteristic length in the $x$-direction to be the length of the meniscus for the static Young-Laplace solution, which is also the half channel width since the Young-Laplace solution has constant curvature. As an alternative to the constant curvature meniscus choice for $x_{c}$ the length of the evaporating thin film up to where it reaches the half-channel height was considered. This length was not chosen because the formulation used in this study for disjoining pressure loses validity with increasing height and curvature. Also, since we will later introduce a thin film model which assumes one-dimensional flow and an interfacial temperature gradient parallel to the wall, there is a loss in physical accuracy as the slope of the interface increases when heading from the thin film to the bulk region. We have also considered scaling at the location where the evaporative mass flux peaks, however, this would relate the characteristic lengths $x_{c}$ and $h_{c}$ via the thin film solution and make them less independent.

The ratio of these characteristic lengths, shown in Equation (5.30), is to be used later for identifying dominant terms in the governing equations and boundary conditions. This will aid in deriving a stability criterion based on the effects of the leading-order physics. 


$$
\tilde{x}=\frac{h_{c}}{x_{c}}
$$

Space is scaled within the film and at the interface according to the ratios

$$
\begin{gathered}
\xi=\frac{x}{x_{c}} \\
\eta=\frac{y}{h_{c}} \\
\tilde{h}=\frac{h}{h_{c}}
\end{gathered}
$$

Time is scaled using the kinematic viscosity and both characteristic lengths, $x_{c}$ and $h_{c}$ according to

$$
\tau=\frac{t \nu}{x_{c} h_{c}}
$$

and we note that any other correct combinations of $x_{c} h_{c}$ could have been used to scale time in Equation (5.34). Pressure and velocity in the $x$ and $y$ directions, respectively are scaled according to ratios

$$
\begin{gathered}
\tilde{P}=\frac{P h_{c}^{2}}{\rho \nu^{2}} \\
\tilde{u}=\frac{u h_{c}}{\nu} \\
\tilde{v}=\frac{v x_{c}}{\nu}
\end{gathered}
$$

We can also scale the temperature, evaporative mass flux, and define the evaporation number noting that $\Delta T=T_{w}-T_{v}$ according to, respectively:

$$
\theta=\frac{T-T_{v}}{\Delta T}
$$




$$
\begin{aligned}
& \tilde{J}=\frac{J h_{c} \mathcal{L}}{k \Delta T} \\
& E=\frac{k \Delta T}{\mu \mathcal{L}}
\end{aligned}
$$

The density ratio between vapour and liquid, surface tension, and surface tension change with temperature are scaled according to, respectively:

$$
\begin{gathered}
D=\frac{\rho_{v}}{\rho} \\
S=\frac{\sigma_{o} h_{c}}{\rho \nu^{2}} \\
C=\frac{\gamma \Delta T}{\sigma_{o}}
\end{gathered}
$$

We introduce the Prandtl number for comparing momentum diffusivity to thermal diffusivity and the Marangoni number for comparing thermocapillary to viscous effects, respectively:

$$
\begin{gathered}
\operatorname{Pr}=\frac{\mu c_{p}}{k} \\
M a=\frac{c_{p} \gamma \Delta T h_{c}^{2}}{x_{c} \nu k}
\end{gathered}
$$

Finally, the disjoining pressure, Equation (2.3), is scaled using

$$
\tilde{\Pi}=\frac{\bar{A}}{\rho \nu^{2} h_{c}}
$$

We now have a set of equations that will be used to scale the governing equations and boundary conditions in terms of the characteristic lengths. The scaled equations below are boxed for convenience. 


\subsubsection{Mass conservation equation scaling}

Mass conservation within the film is scaled by substituting Equations $(5.31,5.32,5.36$, and 5.37) into Equation (5.9) to obtain Equation (5.47b) or

$$
\begin{array}{r}
\tilde{u}_{\xi} \frac{\nu}{x_{c} h_{c}}+\tilde{v}_{\eta} \frac{\nu}{x_{c} h_{c}}=0 \\
\tilde{u}_{\xi}+\tilde{v}_{\eta}=0
\end{array}
$$

\subsubsection{Momentum conservation equation scaling}

The scaled $x$-momentum balance derived in Equation (5.11) is

$$
\tilde{u}_{\tau} \frac{\nu^{2}}{x_{c} h_{c}^{2}}+\tilde{u} \tilde{u}_{\xi} \frac{\nu^{2}}{x_{c} h_{c}^{2}}+\tilde{v} \tilde{u}_{\eta} \frac{\nu^{2}}{x_{c} h_{c}^{2}}=-\tilde{P}_{\xi} \frac{\nu^{2}}{x_{c} h_{c}^{2}}+\tilde{u}_{\xi \xi} \frac{\nu^{2}}{x_{c}^{2} h_{c}}+\tilde{u}_{\eta \eta} \frac{\nu^{2}}{h_{c}^{3}}
$$

Next we multiply Equation (5.48) by $h_{c}^{3} / \nu^{2}$ and combining with Equation (5.30) to obtain

$$
\tilde{x} \tilde{u}_{\tau}+\tilde{x} \tilde{u} \tilde{u}_{\xi}+\tilde{x} \tilde{v} \tilde{u}_{\eta}=-\tilde{x} \tilde{P}_{\xi}+\tilde{x}^{2} \tilde{u}_{\xi \xi}+\tilde{u}_{\eta \eta}
$$

Scaling the momentum balance in the $y$-direction of Equation (5.12) results in

$$
\tilde{v}_{\tau} \frac{\nu^{2}}{x_{c}^{2} h_{c}}+\tilde{v} \tilde{v}_{\eta} \frac{\nu^{2}}{x_{c}^{2} h_{c}}+\tilde{u} \tilde{v}_{\xi} \frac{\nu^{2}}{x_{c}^{2} h_{c}}=-\tilde{P}_{\eta} \frac{\nu^{2}}{h_{c}^{3}}+\tilde{v}_{\xi \xi} \frac{\nu^{2}}{x_{c}^{3}}+\tilde{v}_{\eta \eta} \frac{\nu^{2}}{x_{c} h_{c}^{2}}
$$

We multiply Equation (5.50) by $h_{c}^{3} / \nu^{2}$ as before to obtain the scaled expression for momentum in the $y$-direction:

$$
\tilde{x}^{2} \tilde{v}_{\tau}+\tilde{x}^{2} \tilde{v} \tilde{v}_{\eta}+\tilde{x}^{2} \tilde{u} \tilde{v}_{\xi}=-\tilde{P}_{\eta}+\tilde{x}^{3} \tilde{v}_{\xi \xi}+\tilde{x} \tilde{v}_{\eta \eta}
$$




\subsubsection{Energy conservation equation scaling}

Scaling of the energy balance in Equation (5.13):

$$
\theta_{\tau} \frac{\nu \Delta T}{h_{c} x_{c}}+\tilde{u} \theta_{\xi} \frac{\nu \Delta T}{h_{c} x_{c}}+\tilde{v} \theta_{\eta} \frac{\nu \Delta T}{h_{c} x_{c}}=\frac{k}{\rho c_{p}}\left(\theta_{\xi \xi} \frac{\Delta T}{x_{c}^{2}}+\theta_{\eta \eta} \frac{\Delta T}{h_{c}^{2}}\right)
$$

We note that the dynamic viscosity is given by $\mu=\rho \nu$ and the Prandtl number by $\operatorname{Pr}=\frac{c_{p} \mu}{k}$ and so that by multiplying Equation (5.52) by $\frac{h_{c}^{2}}{\Delta T_{\mathrm{lv}}}$, the final version of the scaled energy equation within the film becomes

$$
\tilde{x} \theta_{\tau}+\tilde{x} \tilde{u} \theta_{\xi}+\tilde{x} \tilde{v} \theta_{\eta}=\frac{1}{P r}\left(\tilde{x}^{2} \theta_{\xi \xi}+\theta_{\eta \eta}\right)
$$

\subsubsection{Dirichlet boundary conditions scaling}

We now scale the wall boundary condition where $y=0$ and $\eta=0$. Constant wall temperature from Equation (5.14a), no slip from Equation (5.55), and no suction or injection from Equation $(5.14 \mathrm{c})$ scale to the following respective dimensionless boundary conditions:

$$
\begin{aligned}
& \theta=1 \\
& \tilde{u}=0 \\
& \tilde{v}=0
\end{aligned}
$$

At the liquid-vapour interface, where $y=h$, we obtain Equation (5.57) as the scaled boundary value for the $y$-coordinate

$$
\eta=\tilde{h}
$$




\subsubsection{Mass flux jump condition scaling}

Scaling the mass flux, as written in Equation (5.18b), gives

$$
\tilde{J} \frac{k \Delta T}{h_{c} L}=\rho\left(-\tilde{h}_{\xi} \tilde{u} \frac{\nu}{x_{c}}+\tilde{v} \frac{\nu}{x_{c}}-\tilde{h}_{\tau} \frac{\nu}{x_{c}}\right)\left[1+\tilde{h}_{\xi}^{2}\left(\frac{h_{c}}{x_{c}}\right)^{2}\right]^{-\frac{1}{2}}
$$

Now substituting Equation (5.30) and Equation (5.40) in Equation (5.58), we obtain

$$
\tilde{J} E=\left(-\tilde{x} \tilde{h}_{\tau}-\tilde{x} \tilde{h}_{\xi} \tilde{u}+\tilde{x} \tilde{v}\right)\left[1+\tilde{x}^{2} \tilde{h}_{\xi}^{2}\right]^{-\frac{1}{2}}
$$

\subsubsection{Normal stress jump condition scaling}

Before balancing the normal stress across the interface, we look at the scaled disjoining pressure in the form of Equation (5.46) and manipulate it to find the unscaled formula in terms of its scaled version as

$$
\Pi=\frac{\tilde{\Pi} \rho \nu^{2}}{\tilde{h}^{3} h_{c}^{2}}
$$

Referring to Equation (5.25), the following scaling is applied:

$$
\begin{aligned}
& \frac{\tilde{J}^{2}}{\rho_{v}}\left(\frac{k \Delta T}{h_{c} \mathcal{L}}\right)^{2}=\tilde{P} \frac{\rho \nu^{2}}{h_{c}^{2}}-\tilde{P}_{v} \frac{\rho \nu^{2}}{h_{c}^{2}}+\tilde{h}_{\xi \xi} \frac{\sigma_{o} h_{c}}{x_{c}^{2}}(1-C \theta)\left[1+\tilde{h}_{\xi}^{2}\left(\frac{h_{c}}{x_{c}}\right)^{2}\right]^{-\frac{3}{2}}+\frac{\tilde{\Pi}}{\tilde{h}^{3}} \frac{\rho \nu^{2}}{h_{c}^{2}} \ldots \\
& +2 \mu\left\{\tilde{u}_{\xi} \frac{\nu}{h_{c} x_{c}}\left[\tilde{h}_{\xi}^{2}\left(\frac{h_{c}}{x_{c}}\right)^{2}-1\right]-\tilde{h}_{\xi} \frac{h_{c}}{x_{c}}\left(\tilde{u}_{\eta} \frac{\nu}{h_{c}^{2}}+\tilde{v}_{\xi} \frac{\nu}{x_{c}^{2}}\right)\right\}\left[1+\tilde{h}_{\xi}^{2}\left(\frac{h_{c}}{x_{c}}\right)^{2}\right]^{-1}
\end{aligned}
$$

We multiply Equation (5.61) by $\frac{h_{c}^{2}}{\rho \nu^{2}}$ to obtain 


$$
\begin{aligned}
& \underbrace{\frac{\tilde{J}^{2}}{\left(\frac{\rho_{v}}{\rho}\right)}}_{D} \overbrace{\left(\frac{k \Delta T}{\mathcal{L} \rho \nu}\right)^{2}}^{E^{2}}=\tilde{P}-\tilde{P}_{v}+\tilde{h}_{\xi \xi} \overbrace{\frac{\sigma_{o} h_{c}}{\rho \nu^{2}}}^{S} \tilde{x}^{2}(1-C \theta)\left[1+\tilde{x}^{2} \tilde{h}_{\xi}^{2}\right]^{-\frac{3}{2}}+\frac{\tilde{\Pi}}{\tilde{h}^{3}} \ldots \\
& +2\left\{\tilde{x} \tilde{u}_{\xi}\left[\tilde{x}^{2} \tilde{h}_{\xi}^{2}-1\right]-\tilde{x} \tilde{h}_{\xi}\left(\tilde{u}_{\eta}+\tilde{x}^{2} \tilde{v}_{\xi}\right)\right\}\left[1+\tilde{x}^{2} \tilde{h}_{\xi}^{2}\right]^{-1}
\end{aligned}
$$

and achieve the final form

$$
\begin{gathered}
\frac{\tilde{J}^{2} E^{2}}{D}=\tilde{P}-\tilde{P}_{v}+\tilde{h}_{\xi \xi} S \tilde{x}^{2}(1-C \theta)\left[1+\tilde{x}^{2} \tilde{h}_{\xi}^{2}\right]^{-\frac{3}{2}} \cdots \\
+\frac{\tilde{\Pi}}{\tilde{h}^{3}}+2 \tilde{x}\left\{\tilde{u}_{\xi}\left[\tilde{x}^{2} \tilde{h}_{\xi}^{2}-1\right]-\tilde{h}_{\xi}\left(\tilde{u}_{\eta}+\tilde{x}^{2} \tilde{v}_{\xi}\right)\right\}\left[1+\tilde{x}^{2} \tilde{h}_{\xi}^{2}\right]^{-1}
\end{gathered}
$$

\subsubsection{Tangential stress jump balance scaling}

We now apply the same logic to scale the tangential momentum equation starting from Equation (5.26f):

$$
\nu \mu\left(\tilde{u} \eta \frac{1}{h_{c}^{2}}+\tilde{v}_{\xi} \frac{1}{x_{c}^{2}}\right)\left(1-\tilde{h}_{\xi}^{2} \frac{h_{c}^{2}}{x_{c}^{2}}\right)-4 \nu \mu \tilde{u}_{\xi} \tilde{h}_{\xi} \frac{1}{x_{c}^{2}}=\frac{-\gamma \Delta T}{x_{c}}\left(\theta_{\xi}+\theta_{\eta} \tilde{h}_{\xi}\right)\left(1+\tilde{h}_{\xi}^{2} \frac{h_{c}^{2}}{x_{c}^{2}}\right)^{\frac{1}{2}}
$$

Now multiplying Equation (5.64) by $\frac{h_{c}^{2}}{\nu \mu}$ and noting that $\frac{M a}{P r}=\frac{\gamma h_{c}^{2} \Delta T}{\nu \mu x_{c}}$ from Equation (5.44) and Equation (5.45), we obtain the final form of the scaled tangential shear balance across the interface:

$$
\left(\tilde{u}_{\eta}+\tilde{x}^{2} \tilde{v}_{\xi}\right)\left(1-\tilde{x}^{2} \tilde{h}_{\xi}^{2}\right)-4 \tilde{x} \tilde{u}_{\xi} \tilde{h}_{\xi}=\frac{-M a}{\operatorname{Pr}}\left(\theta_{\xi}+\theta_{\eta} \tilde{h}_{\xi}\right)\left(1+\tilde{x}^{2} \tilde{h}_{\xi}^{2}\right)^{\frac{1}{2}}
$$




\subsubsection{Energy jump condition scaling}

We can scale Equation (5.28b) to obtain Equation (5.66b):

$$
\begin{aligned}
\tilde{J} \frac{k \Delta T}{h_{c} \mathcal{L}} \mathcal{L} & =k\left(\frac{\Delta T}{x_{c}} \theta_{\xi} \tilde{x} \tilde{h}_{\xi}-\frac{\Delta T}{h_{c}} \theta_{\eta}\right)\left(1+\tilde{x}^{2} \tilde{h}_{\xi}^{2}\right)^{-\frac{1}{2}} \\
\tilde{J} & =\left(\tilde{x}^{2} \tilde{h}_{\xi} \theta_{\xi}-\theta_{\eta}\right)\left(1+\tilde{x}^{2} \tilde{h}_{\xi}^{2}\right)^{-\frac{1}{2}}
\end{aligned}
$$

\subsubsection{Constitutive equation scaling}

Given the dimensional nature of interfacial resistance $K\left(\mathrm{~K} \mathrm{~s} \mathrm{~m}^{2} \mathrm{~kg}^{-1}\right)$, we find the scaling factor by substituting the scaled temperature, $\theta$ and the scaled mass flux, $\tilde{J}$ in Equation (2.15) to obtain Equation (5.67b):

$$
\begin{aligned}
K & =\frac{\theta}{\tilde{J}} \frac{h_{c} \mathcal{L}}{k} \\
\tilde{K} & =\frac{\theta}{\tilde{J}}
\end{aligned}
$$

\subsection{Leading-order approximation}

The derived scaled governing equations and boundary conditions contain numerous terms which do not all have an equivalent impact on the thin film solution. For analytical clarity, we will simplify the previously derived equations to their leading-order terms before formulating a solution. 


\subsubsection{Power series expressions of variables}

We can write the dimensionless variables for the governing equations in terms of a converging series in $\tilde{x}$ keeping in mind that $\tilde{x} \ll 1$ :

$$
\begin{aligned}
& \tilde{u}=U_{o}+\tilde{x} U_{1}+\tilde{x}^{2} U_{2}+\ldots \\
& \tilde{v}=V_{o}+\tilde{x} V_{1}+\tilde{x}^{2} V_{2}+\ldots \\
& \tilde{J}=J_{o}+\tilde{x} J_{1}+\tilde{x}^{2} J_{2}+\ldots \\
& \theta=\theta_{o}+\tilde{x} \theta_{1}+\tilde{x}^{2} \theta_{2}+\ldots \\
& \tilde{P}=\tilde{x}^{-1} P_{o}+P_{1}+\tilde{x} P_{2}+\ldots
\end{aligned}
$$

The numerically subscripted terms in the series above are functions of $\xi, \eta$, and $\tau$. To simplify the problem, we assume that $\{\tilde{u}, \tilde{v}, \tilde{J}, \theta\} \approx \mathcal{O}(1)$ and $\tilde{P} \approx \mathcal{O}\left(\tilde{x}^{-1}\right)$. As such, the scaled velocity parallel to the wall, velocity normal to the wall, evaporative mass flux, temperature, and pressure can be approximated using the leading-order terms in the power series above:

$$
\begin{aligned}
& U_{o} \approx \tilde{u} \\
& V_{o} \approx \tilde{v} \\
& J_{o} \approx \tilde{J} \\
& \theta_{o} \approx \theta \\
& P_{o} \approx \tilde{x} \tilde{P}
\end{aligned}
$$


We will now substitute the leading-order approximations for the dimensionless variables into the governing equations.

\subsubsection{Leading-order governing equations}

Equations $(5.47 \mathrm{~b}, 5.49,5.51$, and 5.53$)$ are written using the leading-order terms given by Equations (5.73-5.77) to yield the following equations for mass continuity, $\xi$-momentum conservation, $\eta$-momentum conservation, and energy conservation, respectively:

$$
\begin{array}{r}
U_{o \xi}+V_{o \eta}=0 \\
-P_{o \xi}+U_{o \eta \eta}=0 \\
P_{o \eta}=0 \\
\theta_{o \eta \eta}=0
\end{array}
$$

\subsubsection{Leading-order boundary conditions}

We now consider the boundary conditions, namely the evaporative mass flux, constitutive resistance to evaporation, normal stress, shear stress, and energy equations.

For the scaled kinematic boundary condition in Equation (5.59), we see that keeping only terms without $\tilde{x}$ as a common factor would lead to a trivial solution, thus we again scale the equation by introducing a scaled evaporation number:

$$
E_{o}=\tilde{x}^{-1} E
$$


Now we can rewrite the newly scaled version of Equation (5.59) using Equation (5.82) and obtain the following, leading-order, equation for the kinematic boundary condition:

$$
E_{o} J_{o}=-\tilde{h}_{\tau}-U_{o} \tilde{h}_{\xi}+V_{o}
$$

The normal stress jump condition in Equation (5.63) is scaled to the leading-order by assigning an order to its constituents and just keeping the leading-order terms. We will now assume the following orders of magnitude for parameters to obtain a leading-order equation.

First, we assume that the disjoining pressure has the same order as the liquid pressure, $\tilde{P}$ scaled in Equation (5.77). Introducing a first order scaled disjoining pressure, $\Pi_{o}$, the following relation is obtained:

$$
\Pi_{o}=\tilde{x} \tilde{\Pi}
$$

The capillary term $C$ which is of assumed order $\mathcal{O}(C)=\tilde{x}$ leads to the introduction of the leading-order term $C_{o}$ such that:

$$
C_{o}=\tilde{x}^{-1} C
$$

The density ratio is assumed $\mathcal{O}(D)=\tilde{x}^{3}$, therefore we can write a leading-order density ratio: $D_{o}$, such that:

$$
D_{o}=\tilde{x}^{-3} D
$$

For the surface tension scaling, the order of $S$ is assumed $\mathcal{O}(S)=\tilde{x}^{-3}$ and we assign its leading-order counterpart, $S_{o}$, such that:

$$
S_{o}=\tilde{x}^{3} S
$$


We now substitute the above scaled terms into Equation (5.63) and obtain:

$$
\begin{array}{r}
\tilde{x}^{-1} \frac{J_{o}^{2} E_{o}^{2}}{D_{o}}=\tilde{x}^{-1} P_{o}-\tilde{x}^{-1} P_{v_{o}}+\tilde{x}^{-1} \tilde{h}_{\xi \xi} S_{o}\left(1-\tilde{x} C_{o} \theta_{o}\right)\left[1+\tilde{x}^{2} \tilde{h}_{\xi}^{2}\right]^{-\frac{3}{2}} \cdots \\
+\frac{\tilde{x}^{-1} \Pi_{o}}{\tilde{h}^{3}}+2 \tilde{x}\left\{\tilde{u}_{\xi}\left[\tilde{x}^{2} \tilde{h}_{\xi}^{2}-1\right]-\tilde{h}_{\xi}\left(\tilde{u}_{\eta}+\tilde{x}^{2} \tilde{v}_{\xi}\right)\right\}\left[1+\tilde{x}^{2} \tilde{h}_{\xi}^{2}\right]^{-1}
\end{array}
$$

After multiplying Equation (5.88) by $\tilde{x}$, we neglect terms in which $\tilde{x}$ remains as a factor to obtain the leading-order momentum jump balance as

$$
P_{o}=P_{v_{o}}-S_{o} \tilde{h}_{\xi \xi}-\frac{\Pi_{o}}{\tilde{h}^{3}}+E_{o}^{2} D_{o}^{-1} J_{o}^{2}
$$

The tangential shear jump condition, from Equation (5.65), is reduced to the leading-order via Equation (5.90) and becomes

$$
U_{o \eta}=\frac{-M a}{\operatorname{Pr}}\left(\theta_{o \xi}+\theta_{o \eta} \tilde{h}_{\xi}\right)
$$

The leading-order energy jump from Equation (5.66b) becomes

$$
J_{o}=-\theta_{o \eta}
$$

Finally, the scaled constitutive Equation (5.67b) written with leading-order terms takes form of

$$
\tilde{K}=\frac{\theta_{o}}{J_{o}}
$$




\subsection{Solving the governing equations}

We now turn our attention to obtaining solutions to the previously derived leading-order governing equations. We solve the energy jump, constitutive relation, momentum jump in the normal and tangential directions, and kinematic jump condition to obtain the evolution equation which describes the change in film height with respect to time. The solution order below is chosen as such because equations first introduced are used to solve for those later in the sequence.

\subsubsection{Energy jump solution}

Solving Equation (5.91) with boundary conditions at $\eta=0$ of $\theta_{o}=1$ and at $\eta=\tilde{h}$ of $J_{o}=-\theta_{o \eta}$ yields

$$
\begin{aligned}
\iint \theta_{o \eta \eta} d \eta d \eta & =c_{1}+c_{2} \eta \\
\theta_{o} & =1-J_{o} \eta \\
\theta_{o} & =1-J_{o} \tilde{h}
\end{aligned}
$$

\subsubsection{Constitutive equation solution}

We solve for $J_{o}$ by substituting the energy jump solution, in Equation (5.93c), into Equation (5.92) to obtain:

$$
J_{o}=\frac{1}{\tilde{K}+\tilde{h}}
$$




\subsubsection{Normal momentum jump solution}

To solve for momentum normal to the interface, we solve Equation (5.80) with the boundary condition in Equation (5.89)

$$
\begin{aligned}
\int P_{o \eta} d \eta & =c_{1} \\
P_{o} & =P_{v_{o}}-S_{o} \tilde{h}_{\xi \xi}-\frac{\Pi_{o}}{\tilde{h}^{3}}+E_{o}^{2} D_{o}^{-1} J_{o}^{2}
\end{aligned}
$$

\subsubsection{Tangential momentum jump solution}

We now solve for the interfacial tangential shear stress from Equation (5.79) and note the term $P_{o \xi}$ can be obtained by differentiating Equation (5.95b) while noting that $P_{v o}$ is constant for our one-sided model and thus disappears with differentiation. Given the constitutive equation solution, $J_{o}=(\tilde{h}+\tilde{K})^{-1}$ from Equation (5.94), we obtain

$$
P_{o \xi}=-S_{o} \tilde{h}_{\xi \xi \xi}+3 \frac{\Pi_{o}}{\tilde{h}^{4}} \tilde{h}_{\xi}-2 E_{o}^{2} D_{o}^{-1}(\tilde{h}+\tilde{K})^{-3} \tilde{h}_{\xi}
$$

Substituting Equation (5.96) into Equation (5.79) yields

$$
U_{o \eta \eta}=-S_{o} \tilde{h}_{\xi \xi \xi}+3 \frac{\Pi_{o}}{\tilde{h}^{4}} \tilde{h}_{\xi}-2 E_{o}^{2} D_{o}^{-1}(\tilde{h}+\tilde{K})^{-3} \tilde{h}_{\xi}
$$

We denote the right hand side of Equation (5.97) as $\Phi(\xi, \tau)$ :

$$
\Phi(\xi, \tau)=-S_{o} \tilde{h}_{\xi \xi \xi}+3 \frac{\Pi_{o}}{\tilde{h}^{4}} \tilde{h}_{\xi}-2 E_{o}^{2} D_{o}^{-1}(\tilde{h}+\tilde{K})^{-3} \tilde{h}_{\xi}
$$

and integrate twice to get 


$$
U_{o}=\frac{1}{2} \Phi(\xi, \tau) \eta^{2}+c_{1} \eta+c_{2}
$$

The boundary conditions are $U_{o}=0$ at $\eta=0$ which makes $c_{2}=0$ and $U_{o \eta}=\frac{-M a}{P r}\left(\theta_{o \xi}+\theta_{o \eta} \tilde{h}_{\xi}\right)$ from Equation (5.90) which is now set equal to a differentiated form of Equation (5.99):

$$
U_{o \eta}=\Phi(\xi, \tau) \eta+c_{1}
$$

Given the energy jump solution: $\theta_{o}=1-J_{o} \eta$ and the constitutive equation solution: $J_{o}=(\tilde{h}+\tilde{K})^{-1}$ we can proceed to differentiate $\theta_{o}$ with respect to both $\eta$ and $\xi$, and use the result to solve for $c_{1}$. Thus,

$$
\begin{aligned}
& \theta_{o \eta}=-(\tilde{h}+\tilde{K})^{-1} \\
& \theta_{o \xi}=-(\tilde{h}+\tilde{K})^{-2} \tilde{h}_{\xi} \tilde{h} \\
& c_{1}=\frac{M a}{\operatorname{Pr}}\left[(\tilde{h}+\tilde{K})^{-1} \tilde{h}\right]_{\xi}-\Phi \tilde{h}
\end{aligned}
$$

after replacing $J_{o}$ using Equation (5.94). We can now solve for $U_{o}$ by substituting Equation (5.101c) into Equation (5.99):

$$
\begin{aligned}
& U_{o}=\frac{1}{2} \Phi \eta^{2}+\left(\frac{M a}{P r}\left[(\tilde{h}+\tilde{K})^{-1} \tilde{h}\right]_{\xi}-\Phi \tilde{h}\right) \eta \\
& U_{o}=\left(\frac{1}{2} \eta^{2}-\tilde{h} \eta\right) \Phi+\frac{M a}{\operatorname{Pr}}\left[(\tilde{h}+\tilde{K})^{-1} \tilde{h}\right]_{\xi} \eta
\end{aligned}
$$


Now having formulated an expression for the velocity parallel to the wall, $U_{o}$, we can use the film mass continuity relation in Equation (5.78) and tangential momentum jump solution in Equation (5.102) to obtain an expression for the speed normal to the wall:

$$
V_{o}=\int\left\{-\Phi_{\xi}\left(\frac{1}{2} \eta^{2}-\tilde{h} \eta\right)+\tilde{h}_{\xi} \Phi \eta-\frac{M a}{P r}\left[(\tilde{h}+\tilde{K})^{-1} \tilde{h}\right]_{\xi \xi} \eta\right\} d \eta
$$

With no suction or injection at the wall, we get zero as an integration constant when solving Equation (5.103) and our final result

$$
V_{o}=-\Phi_{\xi}\left(\frac{1}{6} \eta^{3}-\frac{1}{2} \tilde{h} \eta^{2}\right)+\frac{1}{2} \tilde{h}_{\xi} \Phi \eta^{2}-\frac{M a}{2 P r}\left[(\tilde{h}+\tilde{K})^{-1} \tilde{h}\right]_{\xi \xi} \eta^{2}
$$

\subsubsection{Film height evolution equation obtained using the interfacial kine- matic condition}

We now look at the kinematic condition in Equation (5.83) and substitute the formulas obtained for $U_{o}, V_{o}$, and $J_{o}$ to obtain the following:

$$
\begin{aligned}
& \tilde{h}_{\tau}+E_{o}(\tilde{h}+\tilde{K})^{-1}-\left(\frac{1}{3} \Phi \tilde{h}^{3}\right)_{\xi}+\left[\frac{M a}{2 P r}\left[(\tilde{h}+\tilde{K})^{-1} \tilde{h}\right]_{\xi} \tilde{h}^{2}\right]_{\xi}=0 \\
& \tilde{h}_{\tau}+E_{o}(\tilde{h}+\tilde{K})^{-1}-\left(\frac{1}{3} \Phi \tilde{h}^{3}\right)_{\xi}+\frac{\tilde{K} M a}{2 P r}\left[(\tilde{h}+\tilde{K})^{-2} \tilde{h}^{2} \tilde{h}_{\xi}\right]_{\xi}=0
\end{aligned}
$$

Substituting the full form of $\Phi=-S_{o} \tilde{h}_{\xi \xi \xi}+3 \frac{\Pi_{o}}{\tilde{h}^{4}} \tilde{h}_{\xi}-2 E_{o}^{2} D_{o}^{-1}(\tilde{h}+\tilde{K})^{-3} \tilde{h}_{\xi}$ into Equation (5.105b) we get the final formulation: 


$$
\begin{aligned}
\tilde{h}_{\tau}=-E_{o}(\tilde{h}+\tilde{K})^{-1} & +\left(\frac{\Pi_{o} \tilde{h}_{\xi}}{\tilde{h}}\right)_{\xi}-\left(\frac{1}{3} S_{o} \tilde{h}^{3} \tilde{h}_{\xi \xi \xi}\right)_{\xi} \ldots \\
& -\frac{2}{3} E_{o}^{2} D_{o}^{-1}\left((\tilde{h}+\tilde{K})^{-3} \tilde{h}^{3} \tilde{h}_{\xi}\right)_{\xi}-\frac{\tilde{K} M a}{2 P r}\left[(\tilde{h}+\tilde{K})^{-2} \tilde{h}^{2} \tilde{h}_{\xi}\right]_{\xi}
\end{aligned}
$$

We now have a single fourth-order differential equation subject uniquely to initial conditions as opposed to a free boundary problem given the motion at the interface due to phase change. This formulation is very similar to the evolution equation first derived in [18]. The first term relates to the change in film height due to evaporation. The second and third term involve the disjoining and capillary pressures, respectively. The fourth term represents the effect of vapour recoil. The last term represents the joined effects of thermocapillarity and viscosity. The height of the film will now be perturbed to determine a criterion describing the stability of an extended meniscus.

\subsection{Stability analysis}

We now move our attention to finding how Equation (5.106) behaves when a perturbation is applied. To eliminate the use of excess notation, we redefine the scaled film height in Equation (5.106) having removed the ' $\sim$ ' overscript which hitherto meant $\tilde{h}=\frac{h}{h_{c}}$ and now we let $h=\tilde{h}$. The form of the perturbation is assumed to follow the form

$$
h^{\prime}(\xi, \tau)=\delta h(\xi, \tau) e^{i \lambda \xi}
$$

where $i$ is the imaginary unit and $\lambda$ is the disturbance wave number which is assumed constant. The space dependence on $\delta h$ is due to the curvature in the film. To simplify the analysis, we neglect the space dependence in the thin film region near the location of the characteristic length where the slope is small and in doing so we reduce Equation (5.107) 
to Equation (5.108):

$$
h^{\prime}(\xi, \tau)=\delta h(\tau) e^{i \lambda \xi}
$$

The exponential describing the perturbation can be separated using Euler's formula,

$$
e^{i x}=\cos (x)+i \sin (x)
$$

into a real and imaginary components. The shape of the film can be described as the sum of its base or unperturbed state and the perturbation such that:

$$
H=\bar{h}+h^{\prime}
$$

where $H$ is the perturbed, non-dimensional film height and $\bar{h}$ is the time dependent base state. Time dependence of $\bar{h}$ is due to the evaporation that occurs regardless of the film's perturbation.

In order to clearly show the perturbation of the thin film described by Equation (5.106), we separate the base state into the following terms for individual consideration:

$$
\begin{aligned}
\underbrace{\bar{h}_{\tau}}_{\mathcal{A}}=\underbrace{-E_{o}(\bar{h}+\tilde{K})^{-1}}_{\mathcal{B}} & +\underbrace{\left(\frac{\Pi_{o} \bar{h}_{\xi}}{\bar{h}}\right)_{\xi}}_{\mathcal{C}}-\underbrace{\left(\frac{1}{3} S_{o} \bar{h}^{3} \bar{h}_{\xi \xi \xi}\right)_{\xi} \ldots}_{\mathcal{D}} \\
& -\underbrace{\frac{2}{3} E_{o}^{2} D_{o}^{-1}\left((\bar{h}+\tilde{K})^{-3} \bar{h}^{3} \bar{h}_{\xi}\right)_{\xi}}_{\mathcal{E}}-\underbrace{\frac{\tilde{K} M a}{2 P r}\left[(\bar{h}+\tilde{K})^{-2} \bar{h}^{2} \bar{h}_{\xi}\right]_{\xi}}_{\mathcal{F}}
\end{aligned}
$$

The mapping operator, $\bar{h} \mapsto H=\bar{h}+h^{\prime}$ was used to show the transformation from the base state to the perturbed state. 


$$
\mathcal{A}: \bar{h}_{\tau} \mapsto \bar{h}_{\tau}+h_{\tau}^{\prime}=\underbrace{\bar{h}_{\tau}}_{\text {unperturbed }}+\delta h_{\tau} e^{i \lambda \xi}
$$

We simplify the terms in $\mathcal{B}$ using the first two terms in the following binomial series [55]:

$$
(1 \pm x)^{-m}=1 \mp m x+\frac{m(m+1)}{2 !} x^{2} \mp \frac{m(m+1)(m+2)}{3 !} x^{3}+\ldots(m>0, \quad|x|<1)
$$

noting that the ' $x$ ' term here is small since $\frac{h^{\prime}}{h} \ll 1$. Thus,

$$
\begin{aligned}
\mathcal{B}:-E_{o}(\bar{h}+\tilde{K})^{-1} & \mapsto-E_{o}\left(\bar{h}+h^{\prime}+\tilde{K}\right)^{-1} \\
& =-E_{o}(\bar{h}+\tilde{K})^{-1}\left(1+\frac{h^{\prime}}{\bar{h}+\tilde{K}}\right)^{-1} \\
& =-E_{o}(\bar{h}+\tilde{K})^{-1}\left(1-\frac{h^{\prime}}{\bar{h}+\tilde{K}}\right) \\
& =-\underbrace{E_{o}(\bar{h}+\tilde{K})^{-1}}_{\text {unperturbed }}+E_{o}(\bar{h}+\tilde{K})^{-2} h^{\prime}
\end{aligned}
$$

The $\mathcal{C}$ term contains derivatives of $\bar{h}$ in $\xi$, hence we solve noting that $h_{\xi}^{\prime}=i \lambda h^{\prime}, h_{\xi \xi}^{\prime}=$ $-\lambda^{2} h^{\prime}$, and more generally $h_{\xi^{n}}^{\prime}=(i \lambda)^{n} h^{\prime}$. Also, we neglect changes in the base state with respect to the $\xi: \bar{h}_{\xi}$, since the slope of the interface is assumed small within the thin film 
near the characteristic height, $h_{c}$.

$$
\begin{aligned}
& \mathcal{C}:\left(\frac{\Pi_{o} \bar{h}_{\xi}}{\bar{h}}\right)_{\xi}=\Pi_{o}\left(\frac{\bar{h}_{\xi \xi}}{\bar{h}}-\frac{\bar{h}_{\xi}^{2}}{\bar{h}^{2}}\right) \\
& \mapsto \Pi_{o}\left[\frac{-\lambda^{2} h^{\prime}}{\bar{h}+h^{\prime}}+\frac{\lambda^{2} h^{2}}{\left(\bar{h}+h^{\prime}\right)^{2}}\right] \\
& =-\lambda^{2} \Pi_{o} h^{\prime}\left[\bar{h}^{-1}\left(\frac{1}{1+h^{\prime} \bar{h}^{-1}}\right)-h^{\prime} \bar{h}^{-2}\left(\frac{1}{\left(1+h^{\prime} \bar{h}^{-1}\right)^{2}}\right)\right] \\
& =-\lambda^{2} \Pi_{o} h^{\prime}\left[\bar{h}^{-1}\left(1-h^{\prime} \bar{h}^{-1}\right)-h^{\prime} \bar{h}^{-2}\left(1-2 h^{\prime} \bar{h}^{-1}\right)\right] \\
& =-\lambda^{2} \Pi_{o} h^{\prime}[\bar{h}^{-1}+\underbrace{2\left(h^{2} \bar{h}^{-3}-h^{\prime} \bar{h}^{-2}\right)}_{H O T}] \\
& =-\lambda^{2} \Pi_{o} \bar{h}^{-1} h^{\prime}
\end{aligned}
$$

$$
\begin{aligned}
\mathcal{D}:-\left(\frac{1}{3} S_{o} \bar{h}^{3} \bar{h}_{\xi \xi \xi}\right)_{\xi} & =-\frac{1}{3} S_{o}\left(3 \bar{h}^{2} \bar{h}_{\xi} \bar{h}_{\xi \xi \xi}+\bar{h}^{3} \bar{h}_{\xi \xi \xi \xi}\right) \\
& \mapsto-\frac{1}{3} S_{o}\left[3\left(\bar{h}+h^{\prime}\right)^{2}\left(i \lambda h^{\prime}\right)\left(i \lambda^{3} h^{\prime}\right)+\left(\bar{h}+h^{\prime}\right)^{3}\left(\lambda^{4} h^{\prime}\right)\right] \\
& =-\frac{1}{3} S_{o}[\underbrace{-3\left(\bar{h}+h^{\prime}\right)^{2} \lambda^{4} h^{\prime 2}}_{H O T}+\left(\bar{h}+h^{\prime}\right)^{3}\left(\lambda^{4} h^{\prime}\right)] \\
& =-\frac{1}{3} S_{o} \lambda^{4} h^{\prime}[\bar{h}^{3}+\underbrace{3 \bar{h}^{2} h^{\prime}+3 \bar{h} h^{\prime 2}+h^{\prime 3}}_{H O T}] \\
& =-\frac{1}{3} S_{o} \lambda^{4} \bar{h}^{3} h^{\prime}
\end{aligned}
$$




$$
\begin{aligned}
\mathcal{E}: & -\frac{2}{3} E_{o}^{2} D_{o}^{-1}\left[(\bar{h}+\tilde{K})^{-3} \bar{h}^{3} \bar{h}_{\xi}\right]_{\xi} \\
& =-\frac{2}{3} E_{o}^{2} D_{o}^{-1}[\underbrace{-3(\bar{h}+\tilde{K})^{-4} \bar{h}_{\xi} \bar{h}^{3} \bar{h}_{\xi}+3(\bar{h}+\tilde{K})^{-3} \bar{h}^{2} h_{\xi}}_{H O T}+(\bar{h}+\tilde{K})^{-3} \bar{h}^{3} \bar{h}_{\xi \xi}] \\
& \mapsto \frac{2}{3} E_{o}^{2} D_{o}^{-1} \lambda^{2}\left[\left(\bar{h}+h^{\prime}+\tilde{K}\right)^{-3} h^{3}\left(\bar{h}+h^{\prime}\right) h^{\prime}\right] \\
& =\frac{2}{3} E_{o}^{2} D_{o}^{-1} \lambda^{2} h^{\prime}\left[(\bar{h}+\tilde{K})^{-3}\left(1+\frac{h^{\prime}}{\bar{h}+\tilde{K}}\right)^{-3}\left(\bar{h}^{3}+H O T\right)\right] \\
& =\frac{2}{3} E_{o}^{2} D_{o}^{-1} \lambda^{2} h^{\prime}\left[(\bar{h}+\tilde{K})^{-3}(1-\underbrace{\frac{3 h^{\prime}}{\bar{h}+\tilde{K}}}_{H O T})^{3}\right] \\
& =\frac{2}{3} E_{o}^{2} D_{o}^{-1} \lambda^{2} \bar{h}^{3}(\bar{h}+\tilde{K})^{-3} h^{\prime}
\end{aligned}
$$

$$
\begin{aligned}
\mathcal{F}: & -\frac{\tilde{K} M a}{2 P r}\left[(\bar{h}+\tilde{K})^{-2} \bar{h}^{2} \bar{h}_{\xi}\right]_{\xi} \\
& \mapsto-\frac{\tilde{K} M a}{2 P r}\left[\left(\bar{h}+h^{\prime}+\tilde{K}\right)^{-2}\left(\bar{h}+h^{\prime}\right)^{2} h_{\xi}\right]_{\xi} \\
& =-\frac{\tilde{K} M a}{2 P r}\left[(\bar{h}+\tilde{K})^{-2}\left(1+\frac{h^{\prime}}{\bar{h}+\tilde{K}}\right)^{-2}\left(\bar{h}^{2}+H O T\right) h_{\xi}\right]_{\xi} \\
& =-\frac{\tilde{K} M a}{2 P r}\left[(\bar{h}+\tilde{K})^{-2}(1-\underbrace{\frac{2 h^{\prime}}{\bar{h}+\tilde{K}}}_{H O T})^{2} h_{\xi}\right]_{\xi} \\
& =\frac{\tilde{K} M a}{2 P r}[-(\bar{h}+\tilde{K})^{-2} \bar{h}^{2} h_{\xi \xi}+\underbrace{2(\bar{h}+\tilde{K})^{-3} \bar{h}^{-2} h_{\xi}^{2}-2(\bar{h}+\tilde{K})^{-2} \bar{h} h_{\xi}^{2}}_{H O T}]
\end{aligned}
$$




$$
=\frac{\tilde{K} M a}{2 P r} \lambda^{2}(\bar{h}+\tilde{K})^{-2} \bar{h}^{2} h^{\prime}
$$

After perturbing the terms $\mathcal{A}-\mathcal{F}$ in the evolution Equation (5.111), we group the terms of the perturbed evolution equation having $h^{\prime}$ in common. Noting the form chosen for the perturbation in Equation (5.108) and taking its time derivative yields:

$$
h_{\tau}^{\prime}=\delta h_{\tau} e^{i \lambda \xi}
$$

We divide the terms in $\mathcal{A}-\mathcal{F}$ by $h^{\prime}$ to form Equation (5.120a). Now substituting Equation (5.119) into Equation (5.120a), we obtain Equation (5.120b):

$$
\begin{aligned}
\frac{\delta h_{\tau} e^{i \lambda \xi}}{h^{\prime}} & =E_{o}(\bar{h}+\tilde{K})^{-2}-\Pi_{o} \bar{h}^{-1} \lambda^{2}-\frac{1}{3} S_{o} \bar{h}^{3} \lambda^{4} \\
& +\frac{2}{3} E_{o}^{2} D_{o}^{-1} \bar{h}^{3}(\bar{h}+\tilde{K})^{-3} \lambda^{2}+\frac{\tilde{K} M a}{2 P r}(\bar{h}+\tilde{K})^{-2} \bar{h}^{2} \lambda^{2} \\
\frac{h_{\tau}^{\prime}}{h^{\prime}} & =E_{o}(\bar{h}+\tilde{K})^{-2}+ \\
& {\left[\frac{2}{3} E_{o}^{2} D_{o}^{-1} \bar{h}^{3}(\bar{h}+\tilde{K})^{-3}-\Pi_{o} \bar{h}^{-1}+\frac{\tilde{K} M a}{2 P r}(\bar{h}+\tilde{K})^{-2} \bar{h}^{2}\right] \lambda^{2} } \\
& -\frac{1}{3} S_{o} \bar{h}^{3} \lambda^{4}
\end{aligned}
$$

where $h_{\tau}^{\prime} / h^{\prime}$ is the perturbation growth rate. The symbol $h^{\prime}$ denotes the film height perturbation and if its change in time, $h_{\tau}^{\prime}$, is of opposite sign, then the perturbation will decrease in amplitude. In contrast, if the sign of the perturbation and its change in time are the same, then the perturbation will grow and destabilise the system.

The wave number $\lambda$ in Equation (5.120b) appears in even powers only, so we can let 
$\Lambda=\lambda^{2}$ and form the following quadratic equation:

$$
\frac{h_{\tau}^{\prime}}{h^{\prime}}=a \Lambda^{2}+b \Lambda+c
$$

where,

$$
\begin{aligned}
a & =-\frac{1}{3} S_{o} \bar{h}^{3} \\
b & =\frac{2}{3} E_{o}^{2} D_{o}^{-1} \bar{h}^{3}(\bar{h}+\tilde{K})^{-3}-\Pi_{o} \bar{h}^{-1}+\frac{\tilde{K} M a}{2 P r}(\bar{h}+\tilde{K})^{-2} \bar{h}^{2} \\
c & =E_{o}(\bar{h}+\tilde{K})^{-2}
\end{aligned}
$$

We note that $a$ is always negative and $c$ is always positive for an evaporating film in Equation (5.122a) and Equation (5.122c), respectively. The evaporation number can be viewed as the ratio of viscous to evaporative time scales [18]. Therefore, if we assume slow evaporation by considering $E_{o} \rightarrow 0$ [26], then when $b$ is negative in Equation $(5.122 \mathrm{~b})$, the growth rate is negative and the following inequality is required for a stable film:

$$
-\Pi_{o} \bar{h}^{-1}+\frac{\tilde{K} M a}{2 P r}(\bar{h}+\tilde{K})^{-2} \bar{h}^{2}<0
$$

Approaching the characteristic height $\bar{h} \rightarrow 1$ and since we expect interfacial destabilisation at the location of the characteristic height, it will be used as the point of analysis for determining the film stability. We now substitute back the disjoining pressure term $\Pi_{o}$ as $\tilde{x} \tilde{\Pi}$, from Equation (5.84), so Equation (5.123) becomes:

$$
\frac{M a}{2 \operatorname{Pr}} \frac{\tilde{K}}{(1+\tilde{K})^{2}}<\tilde{x} \tilde{\Pi}
$$

We now dimensionalise all the elements except $\tilde{K}$ in Equation (5.124), using Equations 
$(5.44,5.45$, and 5.46$)$, to obtain the following inequality:

$$
\Delta T<\frac{2(1+\tilde{K})^{2}}{\tilde{K}} \frac{\bar{A}}{\gamma h_{c}^{2}}
$$

In practical terms, we want to find the minimum superheat that can destabilise an evaporating liquid film, so we differentiate $\frac{(1+\tilde{K})^{2}}{\tilde{K}}$ and set $\frac{d}{d \tilde{K}}\left(\frac{(1+\tilde{K})^{2}}{\tilde{K}}\right)=0$ to find its extremum, and demonstrate that $\frac{d^{2}}{d \tilde{K}^{2}}\left(\frac{(1+\tilde{K})^{2}}{\tilde{K}}\right)>0$ at this extremum which indicates we have found a weak minimum. Thus, performing the differentiation we obtain

$$
\begin{aligned}
\frac{d}{d \tilde{K}}\left(\frac{(1+\tilde{K})^{2}}{\tilde{K}}\right) & =\frac{\tilde{K}^{2}-1}{\tilde{K}^{2}} \\
0 & =\frac{\tilde{K}^{2}-1}{\tilde{K}^{2}} \\
\tilde{K} & = \pm 1
\end{aligned}
$$

Realising that $\tilde{K}>0$ since it represents the interfacial thermal resistance which cannot be negative, we therefore have an extremum at $\tilde{K}=1$. Taking the second derivative to find the type of extremum, we obtain

$$
\begin{aligned}
\frac{d^{2}}{d \tilde{K}^{2}}\left(\frac{(1+\tilde{K})^{2}}{\tilde{K}}\right) & =\frac{2}{\tilde{K}^{3}}, \quad \tilde{K}=1 \\
& =2
\end{aligned}
$$

which demonstrates that the solution $\tilde{K}=1$ is a local, weak minimum [56]. Substituting 
$\tilde{K}=1$ into Equation (5.125) allows us to obtain

$$
\Delta T<\frac{8 \bar{A}}{\gamma h_{c}^{2}}
$$

The inequality in Equation (5.128) indicates that the film will be stable if the superheat is less than the quantity on the right. If the superheat is larger than the quantity on the right, then the perturbation growth rate is positive and a perturbed system will destabilise. We note that the stability criterion does not directly depend on $x_{c}$. In the following chapter, we will develop a thin film solution to find $h_{c}$ and determine the minimum superheat that can destabilise the thin film. 


\section{Chapter 6}

\section{Comparison between theory and}

\section{experiment}

We now develop an evaporating thin film model to use in conjunction with the developed stability criterion to determine the thin film profile and minimum superheat which can theoretically destabilise the meniscus. We use the thin film model to quantify the characteristic length, $h_{c}$ required for our stability analysis. We then calculate a theoretical temperature gradient to compare with the experimental temperature gradient across the meniscus and use this comparison to predict stability.

\subsection{Mathematical modelling of an evaporating meniscus}

The physics related to a thin evaporating liquid film in contact with two infinite parallel flat substrates in close proximity will be discussed. Symmetry between the substrates is assumed allowing for half of the physical system to be modelled. Liquid flow is assumed one-dimensional in the direction parallel to the substrate. The effects of radiation are ignored as the temperatures analysed are relatively low. 


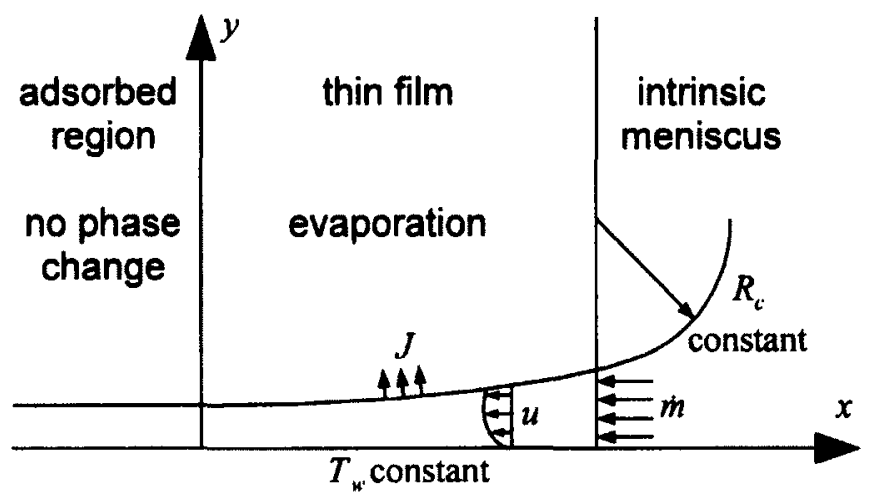

Figure (6.1): Extended meniscus containing the thin film

\subsubsection{Interfacial forces}

The shape of the film is determined by the effects of intermolecular forces between the solid substrate and fluid liquid and gaseous phases resulting in disjoining and capillary pressures. The extended meniscus sketched in Figure (6.1) and can be described by three sections according to intermolecular force variation. The adsorbed region is thin enough such that the attraction between the solid and liquid is strong enough to prevent evaporation. This region is a few nanometers thick and considered to be a continuum. The lack of evaporation results in an interfacial slope of zero which implies null capillary pressure. Upstream of the adsorbed layer is the thin film region wherein evaporation occurs normal to the interface. This film is thin enough such that disjoining pressure effects are significant. Curvature also develops upstream signifying that there is capillary pressure jump across the liquidvapour interface. The effects of disjoining pressure become negligible in comparison to capillary pressure as the film thickens and eventually the interfacial curvature becomes almost constant, but not exactly since a small, yet quantifiable disjoining pressure and evaporative mass flux is present. We call this region the intrinsic meniscus where the larger film thickness has an insulating effect between the heated substrate and vapour. Upon an element of unit area with thickness spanning across the interface, a force balance is applied 
and gives the augmented Young-Laplace formulation

$$
P_{v}-P_{l}=\sigma \kappa+|\Pi|
$$

where $\kappa=1 / R_{1}$ is the interfacial curvature and $\Pi$ is the disjoining pressure. We note the absolute value of the disjoining pressure is used since disjoining pressure tends to decrease the local liquid pressure in the same way as capillary pressure, thus increasing the pressure difference between vapour and liquid across the interface.

\subsubsection{Flow within the thin film}

We use a one-sided model to describe the liquid momentum as not interacting with the vapour above. This is because the vapour's density, viscosity, and thermal conductivity are much smaller than its liquid counterpart. We now drop the subscript indicating a liquid property since we are only dealing with the liquid phase outside the interface. Considering the steady bulk motion of liquid from the intrinsic meniscus to the adsorbed layer, we apply the incompressible mass continuity according to Equation (5.10) from the previously derived stability theory. The steady Navier-Stokes equations for constant density and viscosity and neglecting gravitational forces, in $x$ and $y$ are given by Equation (6.2) and Equation (6.3), respectively and with reference to Figure (6.1):

$$
\begin{gathered}
\rho\left(u u_{x}+v u_{y}\right)=-P_{x}+\mu\left(u_{x x}+u_{y y}\right) \\
\rho\left(u v_{x}+v v_{y}\right)=-P_{y}+\mu\left(v_{x x}+v_{y y}\right)
\end{gathered}
$$


Continuity in Equation (5.10) for steady unidirectional flow in $x$ results in $u_{x}=0$ and $u_{x x}=0$. Equation (6.2) and Equation (6.3) respectively reduce to

$$
\begin{aligned}
& P_{x}=\mu u_{y y} \\
& P_{y}=0
\end{aligned}
$$

Observing Equation (6.5), we note the pressure in the film is constant in the vertical direction so we can treat Equation (6.4) ordinarily and integrate it twice with respect to $y$ to solve for the velocity profile. The constants of integration are obtained via the boundary conditions. At the solid wall, we prescribe no slip as

$$
u(0)=0
$$

At the liquid-vapour interface, we note there is a surface tension gradient as temperature varies. We approximate the surface tension as acting in the $x$ direction allowing us to equate shear to the surface tension gradient in the $x$ direction. Taking the surface tension derivative in $x$ and equating to the shear of a Newtonian fluid at the liquid vapour interface gives its boundary condition

$$
\mu u_{y}=-\gamma\left(T_{l v}\right)_{x}
$$

We can now obtain a function for the velocity by integrating Equation (6.4) twice and using the boundary conditions in Equations (6.6) and (6.7) to obtain

$$
u=\frac{1}{\mu} P_{x}\left(\frac{y^{2}}{2}-h y\right)-\frac{\gamma}{\mu}\left(T_{l v}\right)_{x} y
$$


We now consider the film mass flow rate

$$
\dot{m}=\int_{0}^{h} \rho u d A
$$

where $d A=d y$ for unit depth and substitute Equation (6.8) into Equation (6.9) to obtain

$$
\dot{m}=-\frac{P_{x} h^{3}}{3 \nu}-\frac{\gamma}{2 \mu}\left(T_{l v}\right)_{x} h^{2}
$$

We note that the local evaporative mass flux, $J$ is equal in magnitude to the change in mass flow rate along the film. Thus, the mass flow at $x$ is obtained by integrating mass flux along the film between the adsorbed region $(x=0)$, and $x$ yielding a relation between mass flux and the pressure gradient via

$$
\int_{0}^{x} J d x=\frac{P_{x} h^{3}}{3 \nu}+\frac{\gamma}{2 \mu}\left(T_{l v}\right)_{x} h^{2}
$$

We now introduce the energy Equation (6.12) for an incompressible fluid with constant thermal conductivity, with no internal heat generation or work, and no viscous dissipation:

$$
\rho c_{p}\left(T_{t}+u T_{x}+v T_{y}\right)=k\left(T_{x x}+T_{y y}\right)
$$

Assuming steady state, temperature in the bulk varying only in $y$, and flow only in the $x$ direction, we obtain

$$
k T_{y y}=0
$$

The boundary conditions chosen are constant a wall temperature:

$$
T(0)=T_{w}
$$


and evaporative heat flux $J \mathcal{L}$ at the interface is equal to the conduction gradient such that:

$$
J \mathcal{L}=-\left.k T_{y}\right|_{y=h}
$$

We can now integrate Equation (6.13) twice using Equations (6.14) and (6.15) as boundary conditions to obtain:

$$
T=T_{w}-\frac{J \mathcal{L}}{k} y
$$

Rearranging Equation (6.16) for the mass flux at the interface we obtain:

$$
J=\frac{k\left(T_{w}-T_{l v}\right)}{h \mathcal{L}}
$$

We can write Equation (6.17) in terms of the mass flow rate at any point $x$ along the film using Equation (6.11) to obtain:

$$
\dot{m}=\int_{0}^{x} \frac{k\left(T_{w}-T_{l v}\right)}{h \mathcal{L}} d x
$$

We now look at mass flux on the molecular level which is affected by the interfacial jump of temperature and pressure. A greater temperature jump implies more evaporation and larger capillary and disjoining pressures tend to suppress evaporation. This can be formulated using the model developed in [57] as follows:

$$
J=\frac{2 \alpha}{2-\alpha}\left(\frac{M}{2 \pi R_{u} T_{l v}}\right)^{1 / 2} \frac{P_{v}}{R_{u} T_{l v}}\left(\frac{M \mathcal{L}}{T_{v}}\left(T_{l v}-T_{v}\right)-V_{l}\left(|\Pi|+P_{c}\right)\right)
$$

where $\alpha$ is the accommodation coefficient, $M$ is the molecular mass, $V_{l}$ is the molar volume, and $R_{u}$ is the universal gas constant. We can now combine the molecular model for 
evaporation with the energy Equation (6.17):

$$
\frac{k\left(T_{w}-T_{l v}\right)}{h \mathcal{L}}=\frac{2 \alpha}{2-\alpha}\left(\frac{M}{2 \pi R_{u} T_{l v}}\right)^{1 / 2} \frac{P_{v}}{R_{u} T_{l v}}\left[\frac{M \mathcal{L}}{T_{v}}\left(T_{l v}-T_{v}\right)-V_{l}\left(|\Pi|+P_{c}\right)\right]
$$

The temperature gradient along the interface can be obtained by differentiating Equation (6.20) and isolating for $\left(T_{l v}\right)_{x}$. The process is described in [58] with the result being

$$
\left(T_{l v}\right)_{x}=\frac{2 T_{v}}{h^{3}}\left\{\frac{h_{x}\left[k\left(T_{l v}^{5 / 2}-T_{w} T_{v}^{3 / 2}\right) \frac{2-\alpha}{2 \alpha}\left(\frac{2 \pi}{M}\right)^{1 / 2} \frac{R_{2}^{3 / 2}}{P_{v} \mathcal{L}} h^{2}-3|\bar{A}| V_{l}\right]+V_{l} \sigma \kappa_{x} h^{4}}{k T_{v}\left(5 T_{l v}^{3 / 2}-3 T_{w} T_{l v}^{1 / 2}\right) \frac{2-\alpha}{2 \alpha}\left(\frac{2 \pi}{M}\right)^{1 / 2} \frac{R_{v}^{3 / 2}}{P_{v} \mathcal{L}}+2 h\left(M \mathcal{L}+V_{l} T_{v} \gamma \kappa\right)}\right\}
$$

We now differentiate the pressure balance across the interface in Equation (6.1) with respect to $x$ and assume the vapour pressure is constant above the meniscus. We use Equations $(6.10,2.3$, and 2.11$)$ for the liquid, disjoining, and capillary pressures, respectively. Isolating for the highest order film thickness derivative gives:

$$
h_{x x x}=\frac{1}{\sigma}\left\{\left(\dot{m} \nu+|\bar{A}| h^{-1} h_{x}\right) 3 h^{-3}\left(1+h_{x}^{2}\right)^{3 / 2}-\sigma_{x} h_{x x}+\frac{3 \sigma h_{x} h_{x x}^{2}}{1+h_{x}^{2}}\right\}
$$

We substitute Equation (6.18) into Equation (6.22) for the mass flow rate and note $\sigma_{x}=$ $-\gamma\left(T_{l v}\right)_{x}$ to obtain the final form for the thin film profile:

$$
h_{x x x}=\frac{1}{\sigma}\left\{\left[\frac{\nu k}{\mathcal{L}} \int_{0}^{x}\left(T_{w}-T_{l v}\right) d x+|\bar{A}| h_{x}\right] 3 h^{-4}\left(1+h_{x}^{2}\right)^{3 / 2}+\gamma\left(T_{l v}\right)_{x} h_{x x}+\frac{3 \sigma h_{x} h_{x x}^{2}}{1+h_{x}^{2}}\right\}
$$

Equation (6.23) is solved using a fifth-order explicit Runge-Kutta algorithm [59] to obtain the thin film profile as a function of superheat. A shooting technique is employed to target the desired far field curvature by varying the initial film and its derivatives. The structure of the MATLAB algorithm used to obtain the thin film solution is shown in Figure (6.2). The process begins by choosing the vapour and wall temperatures bounding the working fluid. 
The thickness of the adsorbed layer can be calculated by solving Equation (6.19) assuming the interface and wall temperature are equal with the mass flux and capillary pressure being zero. Values are assigned to the initial film thickness and its derivatives to get the solution started. The solution then marches upstream in $x$. An interface temperature is assumed to solve the film profile and the mass flow difference in Equation (6.18) from the previous step is compared to the evaporative mass flux obtained in Equation (6.19). After matching the mass flux from the macroscopic energy equation to the microscopic evaporation theory, we march upstream and continue the process until $\kappa_{x} \approx 0$. Once near constant curvature is attained as we march in $x$, it is compared the prescribed curvature. If the curvatures do not fall within tolerance, new initial condition values are chosen and the above process is repeated until a match is made. More specifically, the value for the first derivative of the film thickness is varied until a solution is found within the search algorithm. Other initial conditions were held constant to obtain a solution. The computational details are shown in [58] and [60]. A flowchart describing the process utilised to determine the thin film solution is shown in Figure (6.2).

\subsection{Thin film profile on the verge of instability}

\subsubsection{Relation of characteristic lengths to stability criterion}

The stability theory which predicts the minimum superheat that could destabilise the meniscus describes it as a function of the characteristic length, $h_{c}$ according to the stability criterion expressed in Equation (5.128). The significance of this height is that it relates to the disjoining pressure which has a stabilising effect on the film. The opposing destabilising element in the criterion comes from the ratio between the Marangoni and Prandtl numbers, $\mathrm{Ma} / \mathrm{Pr}$. 


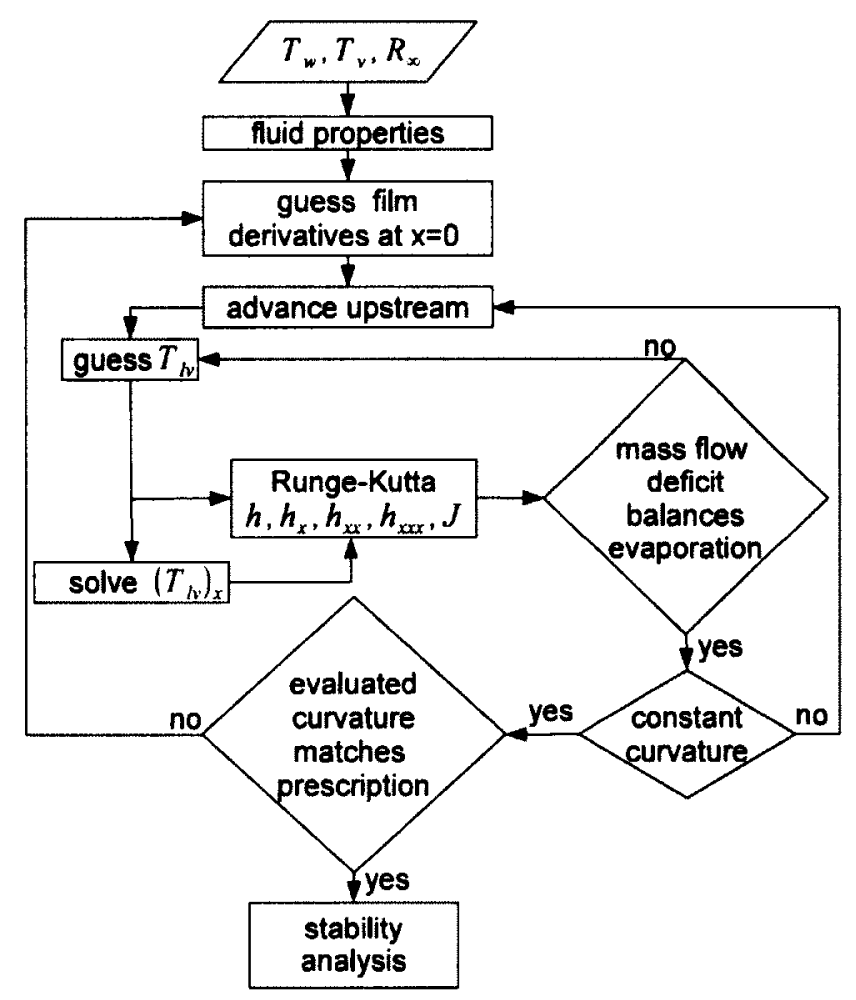

Figure (6.2): Thin film profile solution algorithm

Considering how the characteristic length $x_{c}$ does not directly appear in the stability criterion for an evaporating thin film, we conclude that having chosen $x_{c}$ to represent the length of the meniscus conforming to the Young-Laplace solution with a contact angle of zero is appropriate.

\subsubsection{Matching the thin film and stability criterion superheats}

The structure of the thin film solution algorithm required superheat as an input parameter and proceeded to yield the characteristic length of $h_{c}$. Once $h_{c}$ was calculated, it was used to obtain the minimum superheat that could destabilise an evaporating thin film according to the stability criterion. An iterative process was used for matching the applied superheat and minimum destabilising superheat. In other words, after the first guess of a superheat, the thin film profile was solved for that superheat and the characteristic height, $h_{c}$ was 


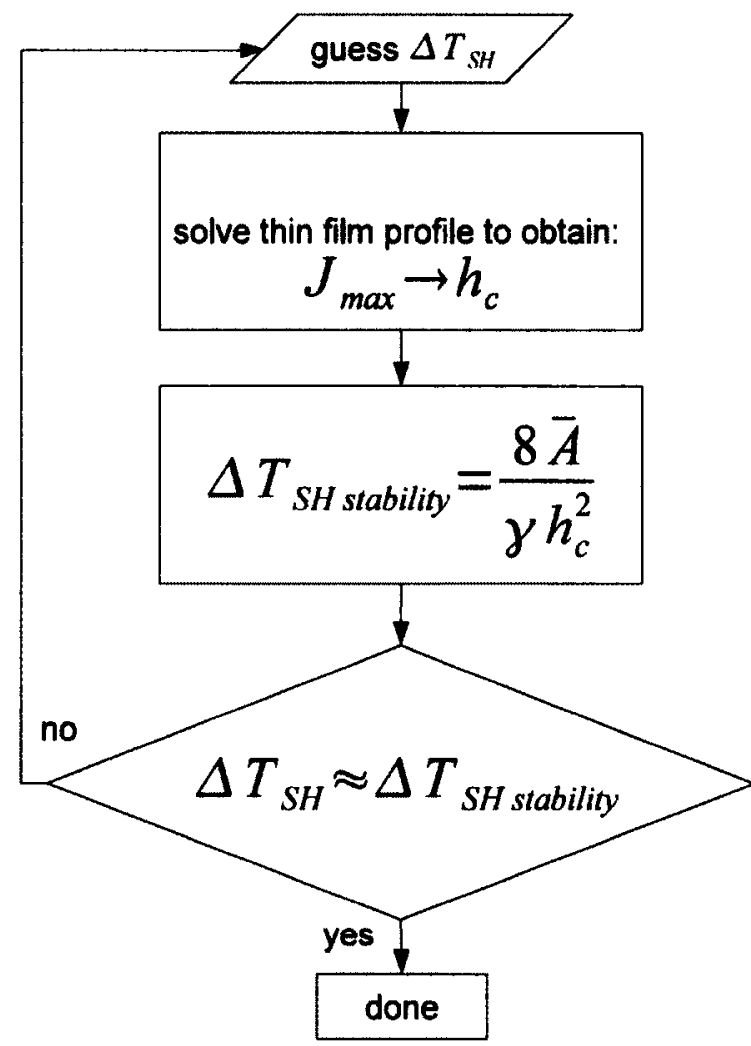

Figure (6.3): Iterative superheat search to match thin film superheat to stability criterion

chosen as where the maximum evaporative heat flux occurred along the film. $\Delta T_{\text {stability }}$ was then calculated using Equation (5.128) and compared with the guess for the superheat. An iterative procedure was followed until the superheat applied to the thin film model matched the one predicted by the Equation (5.128). A flow chart which describes the iterative search

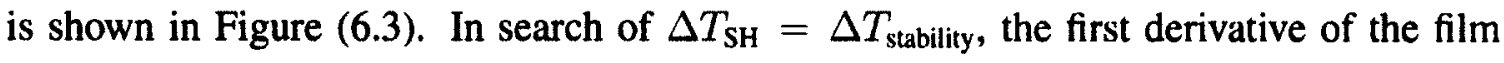
height was varied to obtain a solution. Upon comparison with the stability criterion, the value for the derivative was either increased or decreased to match the film and stability criterion superheats. Solving the thin film model described above for a film on the verge of instability, we can quantify the characteristic length, $h_{c}$ for a $1.0 \mathrm{~mm}$ channel width as shown in Figure (6.4). We note that the characteristic length, $h_{c}$ is the film height where maximum evaporation occurs and it is the critical point where we expect the meniscus to 


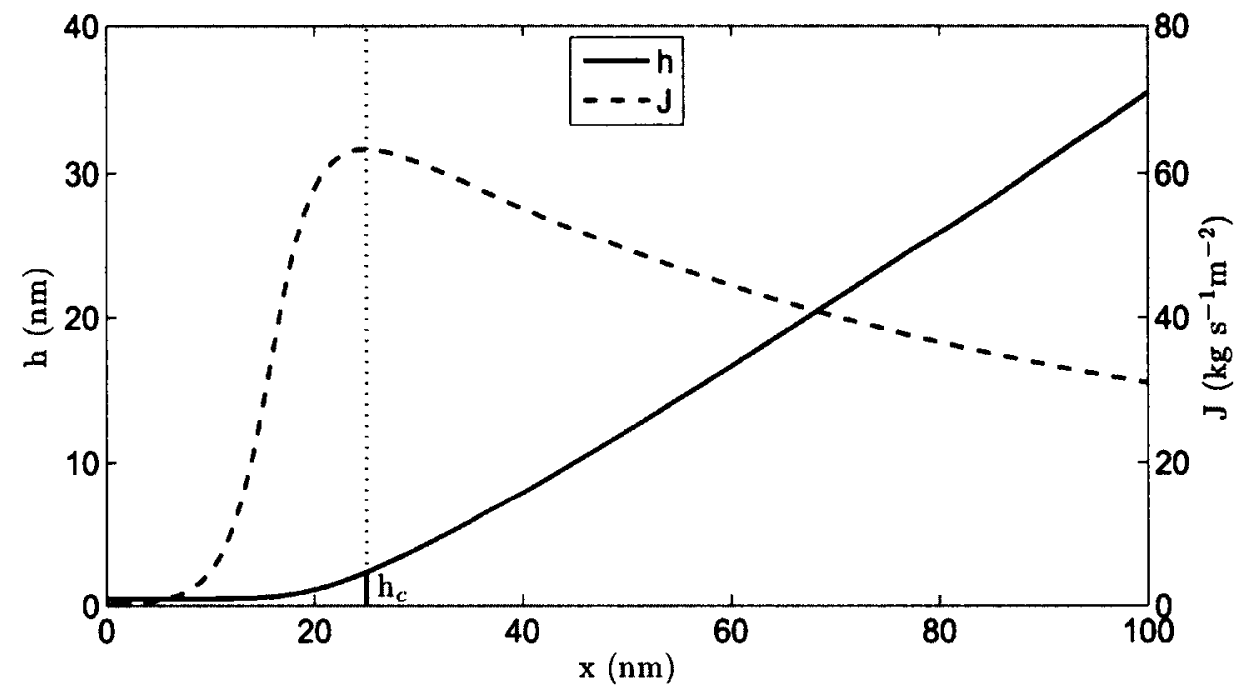

Figure (6.4): Thin film profile, $R_{\infty}=0.5 \mathrm{~mm}$ with associated evaporative mass flux: $J$, characteristic length: $x_{c}=0.5 \mathrm{~mm}$, and $\Delta T_{\mathrm{SH}} / x_{c}=13.0 \mathrm{~K} / \mathrm{mm}$

destabilise.

\subsection{Quantitative comparison of theory with experiment}

Since we cannot directly measure the true superheat in the thin film region due to its small size, moving interface, and sensitivity, it seems that a comparison of the experimentally measured temperature gradient could be more practical. For this, we use the characteristic length in the $x$ direction as it was introduced in Section (5.3) of the stability analysis. Since we are trying to develop a criterion, we are free to choose any form, but its choice should reflect the physics that relate to the destabilisation of the meniscus if the criterion is to have some predictive ability. Generally, the comparison takes the form of the following functional relationship:

$$
f\left(\Delta T_{\mathrm{SH}}, x_{c}\right) \perp \frac{\Delta T_{\text {exp }}}{L}
$$


where $\perp$ is mathematical symbol for comparability. A simple comparison one can make is a direct gradient comparison of the experimental gradient in Equation (6.24) to its theoretical counterpart. We thus compare the experimental temperature gradient between the thermocouples above and below the meniscus, $\Delta T_{\exp } / L$ to the minimum theoretical superheat calculated using the stability criterion, $\Delta T_{\mathrm{SH}} / x_{c}$, where $x_{c}$ is the characteristic length representative of the entire meniscus. Since the stability theory predicts the minimum superheat to destabilise the meniscus, the theoretical gradient is expected to be less than the experimental gradient if a direct gradient comparison is predictive such that:

$$
\frac{\Delta T_{\mathrm{SH}}}{x_{c}}<\frac{\Delta T_{\exp }}{L}
$$

The temperature gradient obtained from experiment is assumed constant between thermocouples. For this assumption to be accurate, the majority of the heat needs to travel down the tube and not into the meniscus. If we also assume the majority of the evaporation occurs in the thin film region as is confirmed by [61], we can neglect evaporation from the intrinsic meniscus and take the thin film evaporation as representative of total evaporation.

For the measurement of the temperature difference across the thermocouples to be representative of the temperature difference at the wall in contact with liquid, the flow of heat should be nearly one-dimensional and in the axial direction. This condition can be satisfied if the wall is sufficiently thin.

We note that it is justifiable to use the Cartesian solution for the stability criterion superheat for comparison with the circular tubes tested since the shape and profile of the thin film is not significantly affected by the circumferential curvature based on the arguments made in [62]. This can also be understood by a simple length scale comparison between the thin film and capillary tube inner diameter. In this study, we can consider the thin film to be less than $100 \mathrm{~nm}$ long. For a tube with a $0.5 \mathrm{~mm}$ diameter, this results in a scale of 
5000:1. Considering how the circumference of the tube appears essentially flat from the perspective of the thin film, we can conclude a thin film on a flat substrate is comparable to one in a circular capillary tube. The small length of the thin film also justifies the use of a model assuming constant wall temperature since the axial temperature change is not significant over such a small distance.

\subsubsection{Significance of temperature gradient comparison for stability prediction}

Since there is a small dependence on far field conditions in the theoretical prediction of the minimum destabilising superheat and we observe an experimental dependency of maximum sustainable temperature gradient on tube inner diameter, we look at the nature of the perturbation which acts on the interface in a speculative attempt to describe this mechanism. The perturbation is modelled as a continuous wave at the interface. The surface can be disturbed either by undesirable factors in the environment such as vibrations or by the energy input to the meniscus via the heater. We note that undesirable environmental factors could act in conjunction with heat input either as a bias which would be undetectable or a random error which could be detected with test repetition. For the three consecutive tests performed for each tube inner and outer diameter combination, no significant random error was detected given how close the temperature gradients were upon the onset of oscillations.

We will only consider the effect of the generated heat as it can be considered present at all time heat is applied, and with increasing amplitude as heat input is increased. Since axial heat conduction in the tube is related to its temperature gradient, it makes sense that an increase in heat to the system would result in a more perturbed state.

As heat input is increased, it drives the flow from the bulk to the thin film. The relative velocity between the liquid and vapour above has a shearing effect at the interface which 
could form a wave in the same way that wind blowing near the surface of a lake can cause a ripple at the surface. Also, given that heat to the film is emanating from a source and transfers axially along the wall, a temperature gradient exists along the wall which contacts the meniscus. A non-uniform temperature is expected at the interface due to the axial temperature gradient. A pull on the interface due to the temperature gradient will again result in relative velocity between the interface and liquid flow. If the axial temperature gradient opposes the liquid pressure gradient as is the case in this experimental study (heating from vapour side), the relative velocity between the interface and liquid flow will be greater than if the temperature gradient and pressure gradient were of the same sign (heating from liquid side).

We now consider how the above waves may be suppressed in the bulk of the meniscus; for this we describe the effect of capillary pressure on the interface. An interface with a significant capillary pressure can be considered analogous to a membrane in tension [63]. More energy is required for a given deflection of a tighter membrane. As an analogy, one can consider the ease with which a partially inflated balloon can be distorted compared to one with higher internal pressure. Likewise, deflecting an interface with higher capillary pressure will require more force than one with lower capillary pressure.

The speculation then becomes that far field perturbations which reach the thin film are more likely with a meniscus in larger diameter capillary tubes, thus a smaller temperature gradient is required for destabilisation compared with smaller diameter capillary tubes. For this mechanism to occur, it is assumed that perturbations start in the bulk region and destabilise the thin film. The converse where perturbations start in the thin film and make their way to the bulk is not assumed. Visual experimental observation confirms stability in the bulk. On the other hand, the oscillatory motion parallel to the wall implies dryout of the thin film as the meniscus drops, while the bulk remains relatively intact. We assume that the perturbation waves in the bulk are undetectable with the optical system in this study 
due to their small size.

As further evidence of the stabilising effect of capillary pressure, we recall in the early stages of testing with water that a stable meniscus was maintained until boiling occurred. This is significant considering the surface tension of water is over four times that of $n$ pentane at the same temperature for the range in this study.

We guess that when the destabilising elements are dominant in the interaction between the stabilising and destabilising mechanisms mentioned above, only then will a wave travel downstream to the thin film region and destabilisation will occur at the location of the characteristic length, $h_{c}$, where evaporative mass flux is highest and dryout is most likely. We note the numerical solution to the evolution equation for an evaporating thin film in [27] also shows perturbations growing in the intrinsic region which lead to rupture in the thin film region.

\subsubsection{Quantifying the experimental and theoretical temperature gra- dients}

The minimum theoretical superheat and temperature gradient to destabilise a thin film between parallel plates is compared with capillary tube testing in Table (6.1).

Table (6.1): Comparison of stability criterion with experimental work with reservoir of $300 \mathrm{~K}$.

\begin{tabular}{cccc}
\hline \hline tube ID & $\begin{array}{c}\text { theory } \\
\Delta T_{\mathrm{SH}} / x_{\mathrm{c}} \\
(\mathrm{K} / \mathrm{mm})\end{array}$ & $\begin{array}{c}\text { experiment thick } \\
\text { tube } \Delta T_{\exp } / L \\
(\mathrm{~K} / \mathrm{mm})\end{array}$ & $\begin{array}{c}\text { experiment thin } \\
\text { tube } \Delta T_{\exp } / L \\
(\mathrm{~K} / \mathrm{mm})\end{array}$ \\
\hline 0.5 & 26.0 & - & $>27.5$ \\
0.6 & 21.7 & 5.71 & - \\
1.0 & 13.0 & - & 10.2 \\
1.2 & 10.8 & 4.58 & - \\
\hline
\end{tabular}


The theoretical criterion trend of a larger temperature gradient being sustainable with decreasing tube inner diameter is reasonable as the capillary pressure effectively stabilises the film as previously discussed. This trend is also observed experimentally as the gradient sustained for the thick tube with $0.6 \mathrm{~mm}$ ID is greater than that for the thick tube with 1.2 $\mathrm{mm}$ ID. Likewise, the thin tube with $0.5 \mathrm{~mm}$ ID was much more stable than the thin tube with $1.0 \mathrm{~mm}$ ID. Destabilisation could not observed with the $0.5 \mathrm{~mm}$ ID tube because of the heater maximum temperature limit, so heat was input until smoke emanating from the heater was observed in the vacuum chamber. The smoke was indicative of deterioration of the carbon composite heater which was confirmed by an increase in the heater electrical resistance measured after the test. The maximum temperature gradient before heater burnout was used as a lower limit approximation for destabilisation.

Upon comparing the thin and thick tubes of close internal diameters, it is evident the gradient for the thin tube is much larger than that for the thick tube. This is primarily because of what is actually measured by the thermocouples attached to the tubes. The higher axial conduction in the thick tube results in a lower temperature difference, but that temperature difference is further away from the meniscus compared to the thin tube measurements. Therefore, the thick tube results are not very representative of what is happening across the meniscus although it is much easier to work using the thick tubes given their robustness. We later abandoned testing with the thick tubes as we achieved the ability to test using thin tubes.

The model presented has some predictive capability when comparing to the thin tube experimental results. The temperature gradient calculated from the $1.0 \mathrm{~mm}$ ID thin tube test was slightly over predicted by the theory, but the results are still satisfactory as a first iteration.

There are several improvements that can be made to increase the predictive ability of 
the model. For the liquid-vapour boundary, a two-sided model which accounts for liquidvapour interaction would help capture behaviour at the interface such as evaporative cooling. At the solid-liquid boundary, a wall temperature gradient could be imposed to better simulate the experimental input.

Several improvements can be made to solving the governing equations. One suggestion is solving the evolution of the thin film in Equation (5.106) and imposing a perturbation numerically. Although this is more computationally intensive, more physics would be captured. If a numerical solution to the evolution equation does not sufficiently predict the instability, a numerical solution for the governing equations and boundary condition derived in this work before simplifying to a leading-order solution in $\tilde{x}$ would eliminate the thin film approximation and validate solving from the intrinsic meniscus to the adsorbed layer. Further refinement could call for the removal of the continuum assumption near the adsorbed layer and using molecular dynamics for its analysis. Such solutions have been published to characterise the thin film as in work in [64], but no work is available on the stability of thin films. The model for evaporation could be refined in such a way that removes the elements of controversy surrounding the evaporation process [47]. 


\section{Chapter 7}

\section{Conclusions and recommendations for}

\section{future work}

\subsection{Conclusions}

The objective of this study was to observe the interfacial behaviour of an evaporating meniscus in a circular capillary tube and to experimentally characterise its stability with energy input, more specifically its axial temperature gradient. A model was introduced to explain the macroscopic experimental findings based on the predictive microscopic thin film behaviour. A stability criterion was developed which was dependent on a characteristic height of the thin film. A thin film model was developed to predict the shape of the thin film at the minimum superheat required for destabilisation according to the derived stability criterion. Characteristic lengths extracted from the model were used in relation to a second chosen criterion to compare with the experimental temperature gradient. The final criterion has shown to have some predictive ability in determining the onset of instability as demonstrated by the comparison of theoretical and experimental results. Subject to further study, this work can be used in the design and analysis of heat pipes, thermosyphons, nuclear 
control rod cooling, inkjet printing, rocket nozzle cooling, and other systems which rely on fluid phase change heat transfer. Of specific ongoing interest is the predictability that can be used for modelling dryout and undesirable temperature fluctuations in heat pipes to help determine operational limits and to create design modifications.

\subsubsection{Experimental conclusions}

\section{Ambient versus vacuum testing}

Testing was first performed in ambient as a stepping stone for later vacuum testing. For initial testing, with the tube exposed to ambient room conditions without any shielding, it was found that temperature would fluctuate to the point where detecting oscillations via temperature variation was severely compromised. The addition of an acrylic enclosure was successful in reducing temperature fluctuations such that temperature variation due to oscillatory behaviour was noticeable.

Although the simplicity of feedthroughs made for easier testing, there was a significant difference in the meniscal physics in air compared to a pure n-pentane environment. As a result, oscillatory behaviour could be induced at a much higher temperature gradient and input power.

\section{Destabilisation for different working fluids}

Early testing with water proved that it was more difficult to destabilise the meniscus due to the higher surface tension of water which stabilised the meniscus against thermal perturbations. 


\section{Meniscus destabilisation with increasing axial temperature gradient}

Increasing the heat load on the meniscus caused its axial temperature gradient to also increase. It was shown that meniscal instability was dependent on the axial temperature gradient across the meniscus.

\section{Thin versus thick tube testing}

Thick and thin tubes, of comparable internal diameters, were used to contain a meniscus to which heat was conducted axially. The thick tubes had thermocouples embedded within their walls to sense temperature closer to the meniscus. Thin tubes had thermocouples epoxied directly onto the outer wall as the wall thickness was much less than its thick counterpart, and therefore not a factor for obtaining accurate temperature measurements. The better axial conduction in the thick tube resulted in a lower temperature gradient and the thermocouples being further from the meniscus made the measurements less representative of the liquid-vapour interfacial physics. As a result, the temperature gradient sensed at the onset of oscillations was smaller for the thick tubes than for the thin tubes. Along with the better axial heat conduction, the thicker tubes allowed for the meniscus to be further away from the heater and were less prone to heater burnout.

\subsubsection{Theoretical conclusions}

The height profile of a curved evaporating thin film evolving in time on a flat plate was found as a fourth-order scaled equation. This equation was subject to a perturbation in height and its stability was formulated as a function of its characteristic height, $h_{c}$.

A thin film model was developed to determine the minimum theoretical superheat that would satisfy the stability criterion developed in the stability theory. To numerically solve for this superheat, the characteristic height of the thin film was chosen as the location of 
maximum evaporation, and this required an iterative solution between the thin film solver and the stability model.

In order to compare the experimental temperature gradient with a theoretical analog, the thin film model superheat was divided by the radius of curvature in the far field to calculate the theoretical temperature gradient. The comparison of experimental and theoretical temperature gradients yielded some level of predictability for the onset of meniscal destabilisation.

\subsection{Recommendations}

\subsubsection{Experimental recommendations}

\section{Parallel plate testing}

Given the Cartesian nature of the theoretical model, it would be fitting to observe the meniscus formed between two flat plates submerged in a reservoir. This is a feasible task given the existing experimental setup which can be adapted for testing various geometries. Plate edge effects can be mitigated by having wide enough plates. This setup is sketched in Figure (7.1) where the heater is centred above the interface peak. Some preliminary work was done in parallel plate testing, but the system was not successfully destabilised. Given the knowledge gained from tube testing, the method for plate testing can be improved with further refinement.

\section{Rectangular tube testing}

Rectangular cross section tubing has also been acquired for further tube testing which can be carried out in a similar manner as that for the circular cross section tubes. The manufacturing of ready to test tubes is essentially identical to round tubes. It is noteworthy 


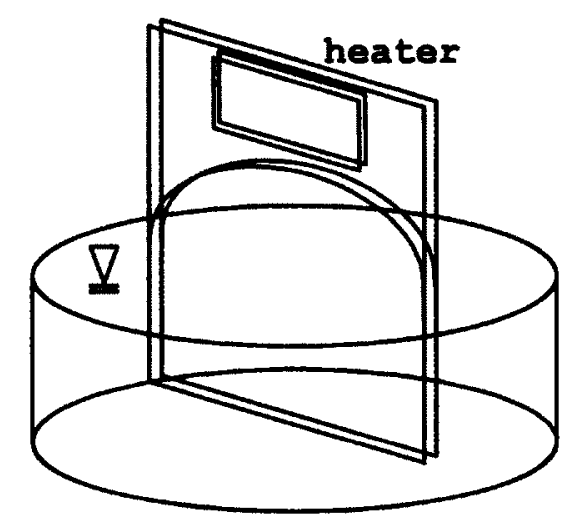

Figure (7.1): Parallel plate testing with heater centred above interface

to mention that rectangular cross section tubes have significant end effects at their corners compared to parallel plates as observed when immersed in an n-pentane bath. Given a number of tubes with different cross-sectional aspect ratios, a parametric study of end effects can be performed to find the minimum length where the physics vary weakly along the length of the cross section at the centre of the tube.

To diminish the curvature at the edges of the rectangular tubes, notches could be made to render the meniscus flatter. The notches could also be used as access ports to measure vapour temperature near the interface.

\subsubsection{Continuous measurement, adjustment, and recording of the reser- voir position}

The dynamic nature of the evaporating meniscus is interesting in that it is affected by the surface tension change with temperature and by the evaporation from both the meniscus inside the capillary tube and the reservoir. Accurate automated positioning of the reservoir could be achieved with a rotary servomechanism that uses the meniscal image as feedback for actuation. The use of a programmable linear stage inside the chamber could also be 
used for more direct control if the stage and test environment can be made compatible.

By tracking the position adjustments made to the reservoir in order to keep the meniscus fixed with respect to the microscope and temperature sensors, an evaporation rate could be experimentally determined. Evaporation from the reservoir alone could be measured without a heated capillary tube.

Image magnification could also be determined by applying a known angular rotation using a servomechanism which could be converted to linear motion used the screw pitch of the linear slide which is equivalent to the linear movement per revolution.

\subsubsection{Leak reduction in the vacuum chamber}

It is inevitable that a system with joints will leak to some extent. By reducing the number of compression and threaded fittings and instead welding components together, the leak rate could be reduced. A helium leak detection test could be performed on the system to pinpoint the biggest leaks.

\subsubsection{Priming of liquid fill line to avoid vapour pulsing into chamber}

When charging the evacuated vacuum chamber, the ambient liquid n-pentane turns to vapour as it experiences a significant pressure drop across the metering valve. As more vapour enters the chamber, its pressure increases until the vapour pressure is reached and then liquid enters the reservoir. Given that this phase change is not global within the system due to temperature variation in the flow and pockets of vapour forming in proximity to liquid, fluid inside the line will be two-phase. Since the system is near saturation throughout testing, this phase change process continues indefinitely being driven by local temperature gradients. Venting the liquid fill line would alleviate vapour pulsing into the reservoir and would also reduce the velocity at which vapour strikes the reservoir for a given charge 


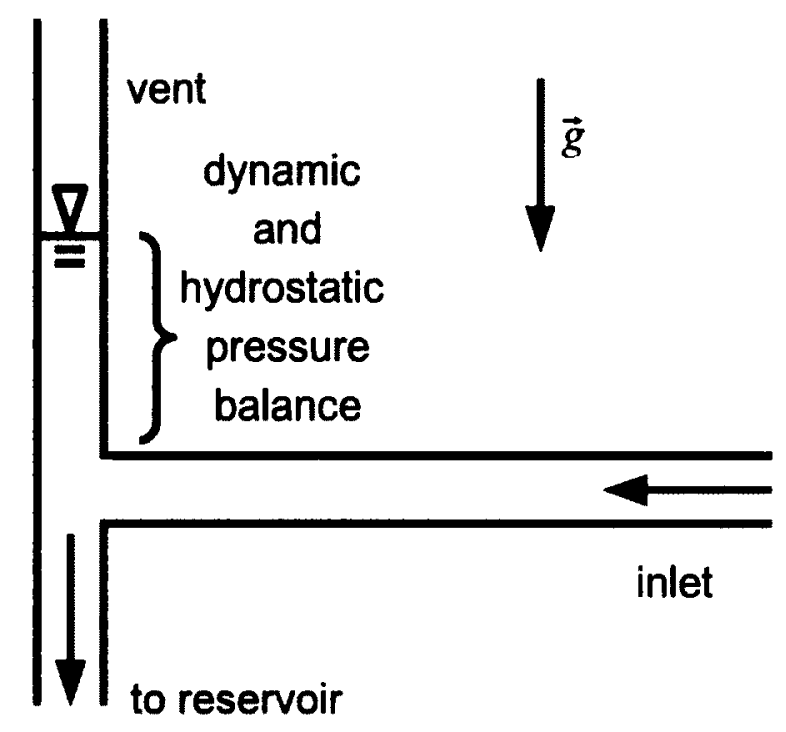

Figure (7.2): Vent for vapour to escape away from reservoir

time before saturation. Venting could be achieved by having two exits for the fluid flowing through the fill line inside the vacuum chamber as shown in Figure (7.2). The one intended for vapour only would point upward away from the reservoir. It would require sufficient height such that the inertia of incoming liquid would not be enough to enter the chamber rising through this line, but would fall down through the liquid line. Before saturation, vapour would be free to enter via both lines, but once liquid enters the chamber, buoyancy would make the upward path preferential for the vapour.

\subsubsection{Chamber rewiring}

Due to the premature degradation of typical electrical wire insulation in an n-pentane vapour environment, the use of Teflon coated wire is recommended for future testing.

\subsubsection{Thermocouple placement jig}

Given the difficulty in affixing thermocouples to the thin walled tubes within a specified distance between leads, a placement jig which holds the thermocouples in a clamp and moves 
them toward the tube could be designed. A small bead of epoxy could then be applied to the leads and the contact pressure between the leads and the tube could be controlled via a leadscrew for each thermocouple. The jig could be made to come apart while applying less force to the leads compared to the current method of removing tape which is used to fix the thermocouples with respect to the tube while the epoxy is curing.

\subsubsection{Measuring wall heat transfer to meniscus}

By accounting for heat applied to the tube and that lost to the environment, the heat transfer from the tube inner wall to the meniscus can be estimated. Quantifying the heat input would help in calculating the evaporative mass flux and thereby give greater insight into the physical system.

\subsubsection{Heater position variation}

In order to determine how the heat input perturbs the meniscus, we propose varying the direction of the heat input temperature gradient with respect to the liquid axial pressure gradient in the meniscus. We note the difficulties associated with water testing in ambient conditions where condensation within the capillary tube would occur when heating from the liquid side. By testing with a volatile hydrocarbon like n-pentane as a working fluid in a saturated environment, we speculate that wall condensation would be greatly reduced, if not eliminated.

\subsubsection{Constant wall temperature testing}

Since the stability model in this study initially assumes a constant wall temperature, it would be fitting to provide heat to the meniscus wall at constant temperature. In order to measure a second temperature to obtain a difference to estimate the superheat, an attempt 


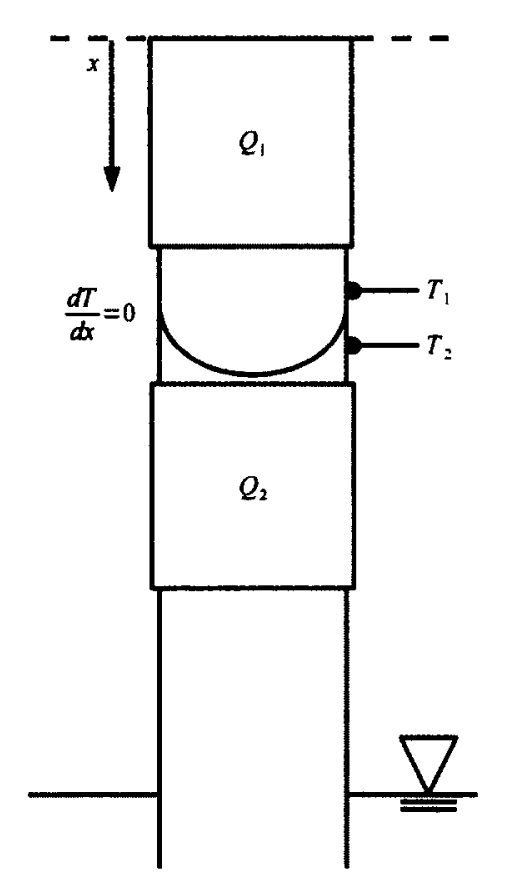

Figure (7.3): Quasi-constant wall temperature near meniscus when using two heaters

could be made to measure the vapour temperature inside the tube via a small thermocouple. We note the time constant of the thermocouple in vapour would be smaller if fixed to the wall, but use of the finest wire feasible would reduce sensing delay. The vapour temperature could also be back calculated by looking at the steady thermocouple response. We note that the application of a constant temperature requires a controller to account for the variation in cooling.

Imposing a near constant wall temperature could be achieved. The first way could be to apply a uniform heat source on the wall which does not rely on axial conduction to transfer heat from the heater to the meniscus. The challenge with this method is that it could potentially hinder meniscus visibility. The use of a radiation source such as a laser to excite the meniscus could be used instead of a conventional resistive heater.

The second method to obtain a quasi-uniform heat would be to heat from both the top and bottom simultaneously with two heaters on one tube as shown in Figure (7.3). If one were to use $T_{1}$ and $T_{2}$ as inputs to a controller using $T_{1}-T_{2} \rightarrow 0$ for control, then the output 
could control the heater power and one could create a near constant temperature about the meniscus. By using a relatively good insulating medium such as quartz to thermally conduct between heaters, a tolerance band around the meniscus could be established as the definition for assumed temperature constancy.

Lastly, one could use a transparent film heater to place over the meniscus. This method is projected to be effective when testing with flat plates as heating surfaces, but it is doubtful that such a heater can be applied on a round capillary with an outer diameter on the order of $1 \mathrm{~mm}$ without transparency issues. The chemical resilience of transparent heaters in a volatile solvent environment should also be determined and improved if necessary and possible.

\subsubsection{Reservoir cooling}

As mentioned before, a means to effectively cool the meniscus could help in testing at higher temperature gradients with lower absolute temperatures which is typically less damaging to the test components. Reservoir temperature variation would also allow for model validation at different reservoir temperatures. The use of a liquid line feedthrough heat exchanger as mentioned in Section (A.8.3) is perhaps the most effective way to cool the incoming test fluid.

\subsubsection{Theoretical recommendations}

\subsubsection{Cylindrical coordinate model}

The modelling of axisymmetric capillary pores is more realistic when comparing with the experimental results for the tubes in this study. The development of a cylindrical model could be beneficial when solving from the bulk region to the thin film. 


\subsubsection{Evaporation model development}

Classical kinetic evaporation theory does not agree closely with experimental findings and there is still a lot of controversy over the details of the evaporation process [47]. The development of a theoretical framework which does not rely so heavily on guesswork such as quantification of the accommodation coefficient should be pursued. Instead of an evaporation model based on classical kinetic theory, a molecular dynamics model could be used to yield a more realistic solution such as the work by Xia and Landman [65] where the evaporation of high temperature alkane films is simulated.

\subsubsection{Unified film and bulk solution}

Assumptions such as one-dimensional liquid flow were made in the thin film model for computational ease, but they lose validity as one approaches the bulk region. For a more thorough understanding of the evaporating meniscus, a solution from the adsorbed region to the bulk is necessary. This would require a more involved computational method as the governing equations and boundary conditions would have to be more general and valid in both film and bulk regions. This approach would come at a loss of elegant, analytical simplicity and it would rely more heavily on numerical methods. Matching the film and bulk solutions would be very computationally demanding. 


\section{References}

[1] R.S. Gaughler. Heat Transfer Device. US Patent 2350348. 1944.

[2] G.M. Grover, T.P. Cotter, and G.F. Erickson. Structures of Very High Thermal Conductance. Journal of Applied Physics, 35(6):1990-1991, 1964.

[3] M.G. Cooper. The Microlayer and Bubble Growth in Nucleate Pool Boiling. International Journal of Heat and Mass Transfer, 12:915-933, 1969.

[4] G.P. Sutton and O. Biblarz. Rocket Propulsion Elements. Wiley, 2001.

[5] E.L. Knuth. The Mechanics of Film Cooling. PhD thesis, California Institute of Technology, 1954.

[6] R. Tsekov, K.W. Stockelhuber, and B.V. Toshev. Disjoining Pressure and Surface Tension of a Small Drop. Langmuir, 16(7):3502-3505, 2000.

[7] R. Tsekov and B.V. Toshev. Capillary Pressure of van der Waals Liquid Nanodrops. Colloid Journal, 74:266-268, 2012.

[8] R. Defay. Surface Tension and Adsorption. Longman and Green, 1966.

[9] T. Cosgorve. Colloid Science. Wiley, 2 edition, 2010.

[10] J.N. Israelachvili. Intermolecular and Surface Forces. Elsevier, 3 edition, 2011. 
[11] B.V. Derjaguin and N.V. Churaev. On the Question of Determining the Concept of Disjoining Pressure and its Role in the Equilibrium and Flow of Thin Films. Journal of Colloid and Interface Science, 66(3), 1978.

[12] V.P. Carey. Liquid-Vapor Phase-Change Phenomena. Taylor and Francis, 2008.

[13] M. Potash, Jr. and P.C. Wayner, Jr. Evaporation From a Two-Dimensional Extended Meniscus. International Journal of Heat and Mass Transfer, 15(3):1851-1863, 1972.

[14] B. Dai and L.G. Leal. Disjoining Pressure for Nonuniform Thin Films. Physical Review E, 76(6), 2008.

[15] R.O. Grigoriev. Control of Evaporatively Driven Instabilities of Thin Liquid Films. Physics of Fluids, 14(6):1895-1909, 2002.

[16] S.G. Bankoff. Dynamics and Stability of Thin Heated Liquid Films. Transactions of the ASME, 112(1):538-546, 1990.

[17] N. Shamsundar and J.H. Lienhard. Equations of State and Spinodal Lines - A Review. Nuclear Engineering and Design, 141:269-287, 1993.

[18] J.P. Burelbach, S.G Bankoff, and S.H. Davis. Nonlinear Stability of Evaporating/Condensing Liquid Films. Journal of Fluid Mechanics, 195:463-494, 1988.

[19] V.K. Badam, V. Kumar, F. Durst, and K. Danov. Experimental and Theoretical Investigations on Interfacial Temperature Jumps During Evaporation. Experimental Thermal and Fluid Science, 32:276-292, 2007.

[20] W. Shyy and R. Narayanan. Fluid Dynamics at Interfaces. Cambridge University Press, 1999. 
[21] D.H. Sharp. An Overview of Rayleigh-Taylor Instability. Physica D: Nonlinear Phenomena, 12(1):3-18, 1984.

[22] K. Adhamkhodaparast, M. Kawaji, and B.N. Antar. The Rayleigh Taylor and Kelvin Helmholtz Stability of a Viscous Liquid Vapor Interface with Heat and Mass Transfer. Physics of Fluids, 7(2):359-364, 1995.

[23] S. Chandrasekhar. Hydrodynamic and Hydromagnetic Stability. Dover, 1981.

[24] L.Y. Leo, R.V. Craster, and O.K. Matar. Marangoni Instability of a Thin Liquid Film Resting on a Locally Heated Horizontal Wall. Physical Review E, 67(5), 2003.

[25] D.H. Michael. Meniscus Stability. Annual Review of Fluid Mechanics, 13:189-215, 1981.

[26] O.E. Shklyaev and E. Fried. Stability of an Evaporating Thin Liquid Film. Journal of Fluid Mechanics, 584:157-183, 2007.

[27] Z. Lichun, M. Tongze, and G. Xinshi. Stability Investigation on the Thin Films in Capillary. Journal of Thermal Science, 10(2):159-164, 2001.

[28] A. Oron, S.H. Davis, and S.G. Bankoff. Long-Scale Evolution of Thin Liquid Films. Review of Modern Physics, 69(3):931-980, 1997.

[29] J. Liu, J.B. Schneider, and J.P. Gollub. Threedimensional Instabilities of Film Flows. Physics of Fluids, 7(1):55-67, 1995.

[30] S. Krishnamoorthy, B. Ramaswamy, and S.W. Joo. Spontaneous Rupture of Thin Liquid Films due to Thermocapillarity: A Full-Scale Direct Numerical Simulation. Physics of Fluids, 7(9):2291-2293, 1995. 
[31] G. Ramon and A. Oron. Capillary Rise of a Meniscus with Phase Change. Journal of Colloid and Interface Science, 327(1):145-151, 2008.

[32] B.V. Zhmud, F. Tiberg, and K. Hallstensson. Dynamics of Capillary Rise. Journal of Colloid and Interface Science, 228(2):263-269, 2000.

[33] G. Preiss and P.C. Wayner, Jr. Evaporation from a Capillary Tube. Journal of Heat Transfer, pages 178-181, May 1976.

[34] J. Pellicer, V. Garcia-Morales, and M.J. Hernandez. On the Demonstration of the Young-Laplace Equation in Introductory Physics Courses. Physics Education, 35(2): 126-129, March 2000.

[35] H.K. Dhavaleswarapu, J.Y. Murthy, and S.V. Garimella. Numerical Investigation of an Evaporating Meniscus in a Channel. International Journal of Heat and Mass Transfer, 55:915-924, 2012.

[36] H.K. Dhavaleswarapu, S.V. Garimella, and J.Y. Murthy. Microscale Temperature Measurements Near the Triple Line of an Evaporating Thin Liquid Film. Journal of Heat Transfer, 131(2), June 2009.

[37] C. Buffone and K. Sefiane. Temperature Measurement Near the Triple Line During Phase Change Using Thermochromic Liquid Crystal Thermography. Experiments in Fluids, 39:99-110, 2005.

[38] C. Buffone and K. Sefiane. IR Measurements of Interfacial Temperature During Phase Change in a Confined Environment. Experimental Thermal and Fluid Science, 29(1):65-74, 2004.

[39] C. Buffone and K. Sefiane. Investigation of Thermocapillary Convective Patterns and 
Their Role in the Enhancement of Evaporation from Pores. International Journal of Multiphase Flow, 30:1071-1091, 2004.

[40] C. Buffone and K. Sefiane. Controlling Evaporative Thermocapillary Convection Using External Heating: an Experimental Investigation. Experimental Thermal and Fluid Science, 32:1287-1300, 2008.

[41] C. Buffone, K. Sefiane, and J.R.E. Christy. Experimental Investigation of the Hydrodynamics and Stability of an Evaporating Wetting Film Placed in a Temperature Gradient. Applied Thermal Engineering, 24:1157-1170, 2004.

[42] C. Buffone, K. Sefiane, and W. Easson. Marangoni-Driven Instabilities of an Evaporating Liquid-Vapor Interface. Physical Review E, 71:056302, May 2005.

[43] C. Buffone, K. Sefiane, and J.R.E. Christy. Experimental Investigation of SelfInduced Thermocapillary Convection for an Evaporating Meniscus in Capillary Tubes using MicroParticle Image Velocimetry. Physics of Fluids, 17:052104, April 2005.

[44] H.J. Palmer and J.C. Maheshri. Enhanced Interfacial Heat Transfer by Differential Vapor Recoil Instabilities. International Journal of Heat and Mass Transfer, 4:117$123,1981$.

[45] S.S. Panchamgam, J.L. Plawsky, and P.C. Wayner, Jr. Microscale Heat Transfer in an Evaporating Moving Extended Meniscus. Experimental Thermal and Fluid Science, 30(8):745-754, August 2006.

[46] D. Pratt. The Effects of Thermocapillary Stresses on the Wetting Characteristics, Heat Transfer Effectiveness, and Stability of an Evaporating, Capillary, Resupplied, Curved Meniscus within a Capillary Tube. $\mathrm{PhD}$ thesis, University of Dayton, 1996. 
[47] P. Rahimi and C.A. Ward. Kinetics of Evaporation: Statistical Rate Theory Approach. International Journal of Thermodynamics, 8(1):1-14, 2005.

[48] Model 7708 40-channel Differential Multiplexer with Automatic CJC Users Guide, 2003.

[49] R.W. Schrage. A Theoretical Study of Interphase Mass Transfer. PhD thesis, Columbia University, 1953.

[50] M.J. Moran and H.N. Shapiro. Fundamentals of Engineering Thermodynamics. Wiley, 4 edition, 2000.

[51] S.J. Kline. The Purposes of Uncertainty Analysis. Journal of Fluids Engineering, 107:153-160, 1985.

[52] R.S. Figliola and D.E. Beasley. Theory and Design for Mechanical Measurement. Wiley, 4 edition, 2006.

[53] Keithley Model 2700 Multimeter/Switch System User's Manual, 2003.

[54] F. White. Viscous Fluid Flow. McGraw-Hill, 1991.

[55] G.A. Korn and T.M. Korn. Mathematical Handbook for Scientists and Engineers. Dover, 1968.

[56] H. Sagan. Introduction to the Calculus of Variations. McGraw-Hill, 1969.

[57] P.C. Wayner, Jr. The Effect of Interfacial Mass Transport on Flow in Thin Liquid Films. Colloids and Surfaces, 52:71-84, 1991.

[58] J. Polansky. Numerical Model of an Evaporating Thin Film. Master's thesis, Carleton University, 2011. 
[59] J.R. Dormand and P.J. Prince. A Family of Embedded Runge-Kutta Formulae. Journal of Computational and Applied Mathematics, 6:19-26, 1980.

[60] G. Ball. Numerical Analysis of the Heat Transfer Characteristics within an Evaporating Meniscus. Master's thesis, Carleton University, 2012.

[61] P.C. Wayner, Jr. Intermolecular Forces in Phase-Change Heat Transfer: 1998 Kern Award Review. American Institute of Chemical Engineers Journal, 45(10):20552068, 1999.

[62] K.P. Hallinan and H.C. Chebaro. Evaporation From an Extended Meniscus for Nonisothermal Interfacial Conditions. Journal of Thermophysics and Heat Transfer, 8(4):709-716, 1994.

[63] K.E. Kenyon. Capillary Waves Understood by an Elementary Method. Journal of Oceanography, 54:343-346, 1998.

[64] S.C. Maroo and J.N. Chung. Heat Transfer Characteristics and Pressure Variation in a Nanoscale Evaporating Meniscus. International Journal of Heat and Mass Transfer, $53: 3335-3345,2010$.

[65] T.K. Xia and U. Landman. Molecular Evaporation and Condensation of Liquid nAlkane Films. Journal of Chemical Physics, 101(3):2498-2507, 1994.

[66] Omega Engineering. Omega Temperature Measurement Handbook, 2007.

[67] L. Ward and Bunn J.P. Introduction to the Theory and Practice of High Vacuum Technology. Butterworths, 1967.

[68] C.I. Young. Bioengineering Heat Transfer. Academic Press, 1992. 
[69] M.F. Gelder. A Thermistor Based Method for Measurement of Thermal Conductivity and Thermal Diffusivity of Moist Food Materials at High Temperatures. $\mathrm{PhD}$ thesis, Virginia Polytechnic Institute and State University, 1998.

[70] Omega Engineering. OB 200 Epoxy Resin; MSDS 0109, 2009.

[71] JB Weld Company. JB Marine Weld Resin; MSDS 8272, 2011.

[72] Aspen Aerogel. Pyrogel 2250 Flexible Insulation for High Temperatures, 2010.

[73] F.P. Incropera, D.P. Dewitt, T.L. Bergman, and A.S. Lavine. Fundamentals of Heat and Mass Transfer. Wiley, 6 edition, 2007.

[74] J.P. Holman. Heat Transfer. McGraw-Hill, 8 edition, 1997.

[75] A. Bejan. Convection Heat Transfer. Wiley, 2 edition, 1995. 


\section{Appendix A}

\section{Details of experimental setup}

\section{A.1 Vibration isolation}

The nature of the testing required isolating the system so that it would not vibrate at a frequency on the order of approximately $1 \mathrm{~Hz}$ produced by a destabilised meniscus. The three variables that were manipulated to isolate the system from unwanted vibrations were mass, stiffness, and damping.

A large mass helps to mitigate vibrational effects by decreasing the acceleration due to an input force. During testing, this was accomplished by using a $240 \mathrm{~kg}$ granite block resting on a steel table; the combination having sufficient inertial resistance so that there would be negligible motion due to typical input forces such as reservoir height adjustments and footsteps.

It was found that having the test bed directly on the floor did not provide adequate vibration isolation due to the uneven and energy transferring nature of the concrete floor. Stiffness was altered via the following methods. The use of inner tubes as air springs to float the granite block was attempted, but proved ineffective as the system would vibrate noticeably during the experimentation process. Anti-vibrational rubber padding with suction 
cups were stacked with sheet metal shims in between to provide grip and an air suspension. This setup being similar to the inner-tube airbag system also did not ease the effects of vibrations.

What proved effective with regard to stiffness was having the test stand supported at all four outer corners using compliant rubber matting. The stiffness of the matting needed to be low enough to allow contact on all patches, but high enough so that the system would not move significantly while subject to input forces.

Damping was also applied to the system by filling the reservoir with $3 \mathrm{~mm}$ diameter glass spheres as shown in Figure (A.1). The additional friction encountered by fluid moving within the reservoir due to increased surface contact area was found to noticeably decrease the amount of time surface undulations were seen after an observer impacted the floor near the test setup in a repeatable manner for comparison.

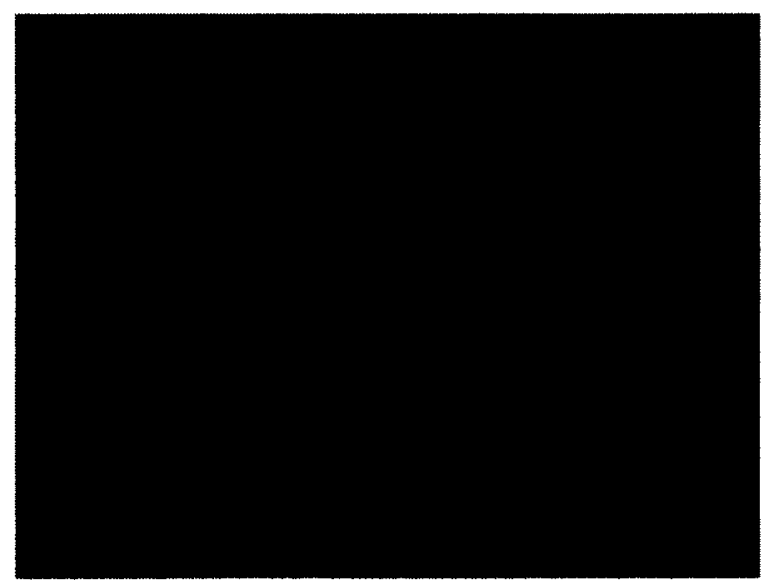

Figure (A.1): The $3 \mathrm{~mm}$ diameter glass beads in a reservoir to dampen vibrations

\section{A.2 Stationary subsystems}

The following is a description of the stationary components of the test infrastructure including: 
- Support bases for the chamber, microscope, and internals within the chamber

- Ambient and vacuum test chambers

- Microscope and scaling reticles

- Data acquisition system

- Feedthroughs for vacuum chamber

\section{A.2.1 Testing support base}

The test base rested on a single concrete slab on the laboratory floor as shown in Figure (3.1). Making contact with the concrete were two strips of rubber matting measuring $61 \mathrm{~cm}$ long by $20 \mathrm{~cm}$ wide by $2 \mathrm{~cm}$ thick. A steel base measuring $108 \mathrm{~cm}$ long by $61 \mathrm{~cm}$ wide by $64 \mathrm{~cm}$ high was rested on the rubber pads located on either edge of the width. A sandbox on top of the base contained a flat surfaced granite block $91 \mathrm{~cm}$ long by $61 \mathrm{~cm}$ wide by $16 \mathrm{~cm}$ high. Finally, the test chamber and microscope were placed at diagonally opposing corners to provide the maximum range for optical focus adjustment.

\section{A.2.2 Testing baseplates}

The external baseplate resided on a rotary table which in turn rested on top of a compound slide which in turn rested on the granite block. The slide-rotary assembly allowed for fine adjustment of the chamber position with respect to the microscope. The external baseplate consisted of an aluminium square optical breadboard $30.5 \mathrm{~cm}$ wide which was bolted to 3 T-slot nuts residing within the rotary table. Four steel legs $20 \mathrm{~cm}$ long were mounted using bolts between the optical breadboard and vacuum chamber floor to provide room for the liquid plumbing, evacuation system, and rotary feedthrough. The internal chamber 
baseplate consisted of an optical breadboard which was bolted onto the chamber floor to provide a mounting surface for the capillary tube bracket post, reservoir mount, and connection sockets for feedthroughs coming from the chamber wall. The chamber floor was custom designed and built to specification out of stainless steel by Kurt J. Lesker Company along with the vacuum chamber bell jar. A circumferential groove in the chamber floor allowed for seating of a centreing ring containing a Viton o-ring for mating the chamber to a ISO 320-K flange. The chamber floor had four blind tapped external holes for mounting to the legs which would provide clearance for the floor feedthroughs, namely; a QF-25 port for connecting the evacuation system, a QF-40 port for the working fluid feedthrough, and a DN-16 CF port for the rotary feedthrough. In exception of the rotary feedthrough using a copper gasket seal, all other baseplate gaskets were either made of Viton or nitrile.

\section{A.2.3 Ambient test chamber}

Initially, testing was performed in an ambient laboratory setting without any shielding from exposure to room conditions. This proved an issue due to air circulation from the building ventilation system. The temperature measured at the thermocouples would fluctuate making it difficult to determine if the system had reached steady state after increasing the power input. This became evident when comparing the temperature fluctuations resulting from testing during the day when the ventilation was on and at night when it was turned off. To block the circulating air, a $3 \mathrm{~mm}$ thick clear acrylic rectangular chamber was constructed with a square base $36 \mathrm{~cm}$ wide, height of $41 \mathrm{~cm}$, and is shown in Figure (A.2). An oversized hole was made to fit the microscope objective allowing for focusing closer to the tube and providing extra clarity. An air guard was hung around the objective to decrease the circulation around the clearance area between the hole and objective.

The dimensions were chosen similar to the vacuum chamber so either could be switched 


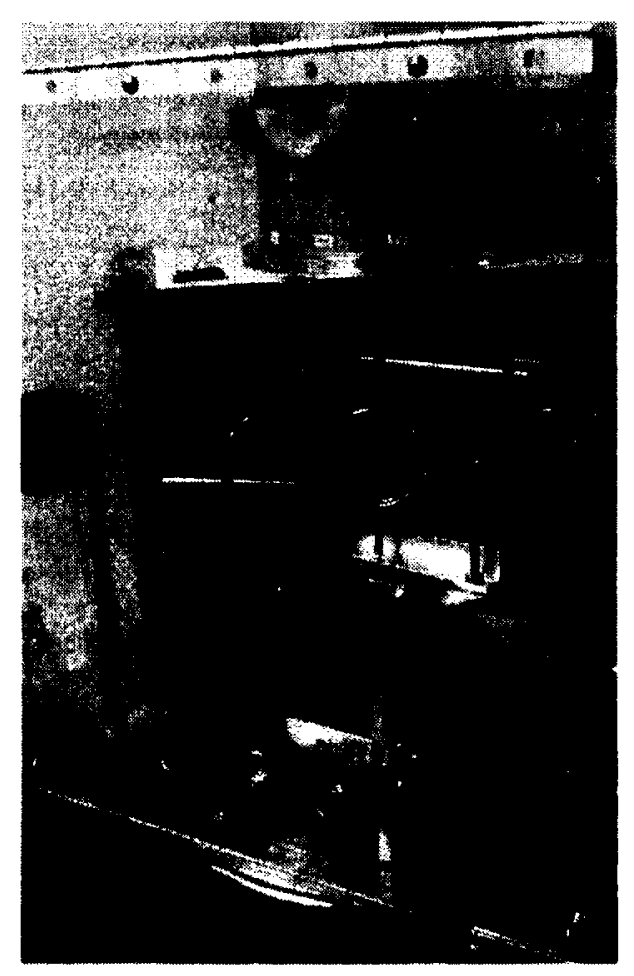

Figure (A.2): Ambient chamber

without having to heavily modify the chamber floor. Access to the ambient test setup without removing the chamber from the floor was made by hanging one of the walls from a piano hinge connected to the chamber roof.

\section{A.2.4 Vacuum test chamber}

An in-house designed vacuum chamber custom built by Kurt J. Lesker Company shown in Figure (A.3) was used for testing. To clarify the discussion which follows, a labeled view of the chamber shown in Figure (A.4) The chamber was $406 \mathrm{~mm}$ in height from the flange to the tip of its cupola roof where a lifting lug was located. The outside wall to wall diameter was $324 \mathrm{~mm}$ with $4.8 \mathrm{~mm}$ wall thickness. A total of three optical windows centred $178 \mathrm{~mm}$ from the flange bottom where used for illumination and observation of the meniscus and height of the reservoir. The three optical ports of type DN-63-CF were sealed using copper 


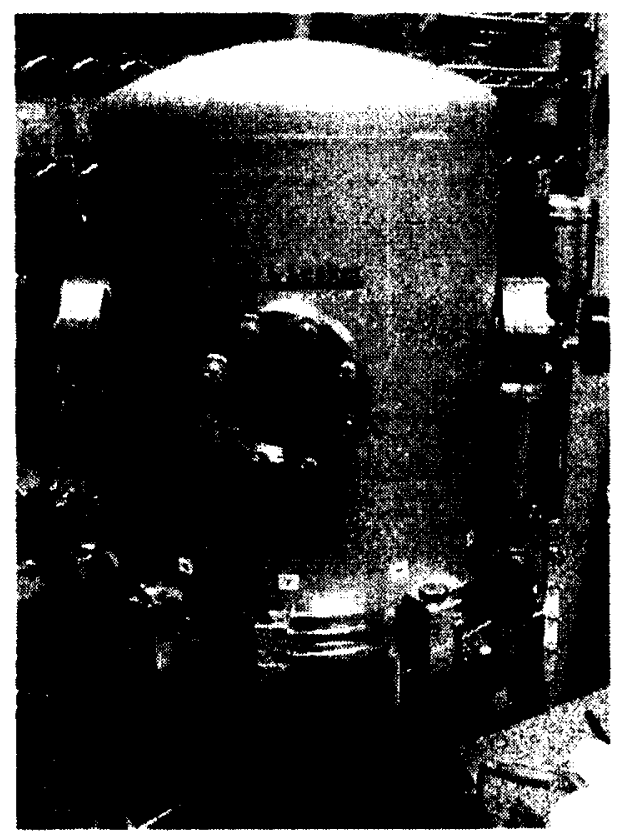

Figure (A.3): Vacuum chamber

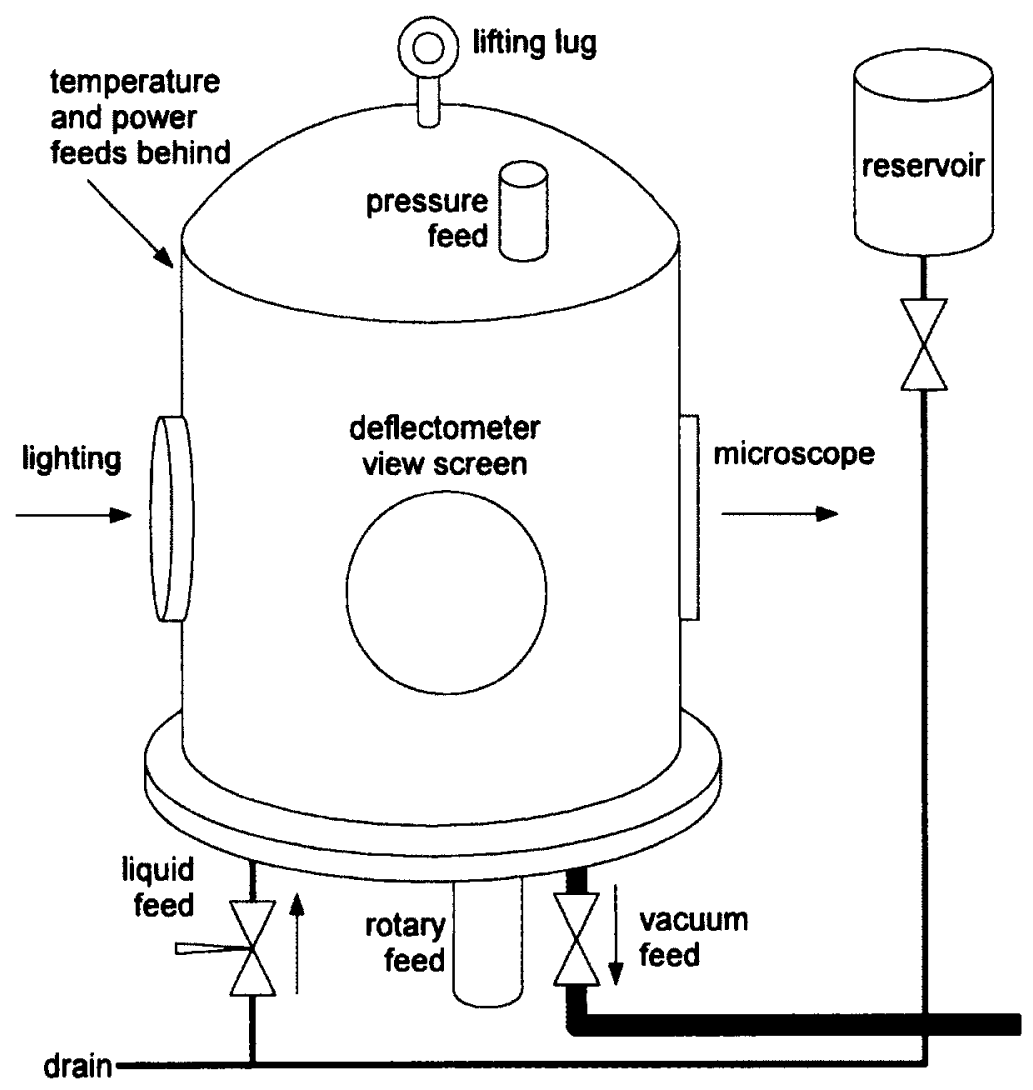

Figure (A.4): External vacuum chamber components 
gaskets separated in sequence by $90^{\circ}$. Two windows in sequence were made from Kodial glass (alkali borosilicate 7056 ) having a $63 \mathrm{~mm}$ diameter and $3 \mathrm{~mm}$ thickness. The window for image capture was made from quartz with a diameter of $61 \mathrm{~mm}$ and a thickness of 4.75 $\mathrm{mm}$. Five QF-40 nitrile o-ring sealed feedthroughs were located on the wall and ceiling of the chamber of which three were used in this study. The two unused feedthroughs were sealed using blank flanges.

\section{A.2.5 Microscope}

An optical stereo microscope (Olympus SZH-10) was used to magnify the meniscus image for recording. The stereo function was not utilised for observation since a monocular camera was used in one eyepiece tube and a reticle was placed in the other. The microscope objective (Olympus DF Planapo 1X) was chosen to accommodate the minimum practical working distance, i.e. the distance between the objective edge and the specimen focal plane where the field of view contains the entire meniscus. A zoom ratio of $0.7 \mathrm{X}$ to $7 \mathrm{X}$ was adjustable with a dial on the microscope body with a click stop option to select set magnification increments for repeatability. The eyepiece containing the reticle provided 10X magnification. Focus was adjusted using a dial to move the assembly parallel to the objective axis of symmetry. An adjustable aperture was kept fully open to maximise light transmission. The locking mechanism to position the microscope is described subsequently.

\section{Microscope ocular reticle}

A scale crossline reticle was selected to provide a reference location for the meniscus to be set with respect to the immobile tube. This was important for placing the meniscus at the same height under the heater in a consistent manner. The reticle had a total of 200 
divisions, with a numbered marking every 10 division, and a division every $0.0635 \mathrm{~mm}$ (0.0025 inch). The reticle was made from instrument quality glass with a $25 \mathrm{~mm}$ diameter, $1.5 \mathrm{~mm}$ thickness, and chrome deposition markings. The reticle resided within the optical pathway between the eyepiece magnifier and microscope zoom.

\section{Lighting}

Illuminating the meniscus to observe its behaviour involved the compromise of providing enough light to see the magnified meniscus, while not adding excessive heat. A highintensity LED (light emitting diode) spotlight (Edmunds NT66-847) providing a white light with $65 \mathrm{~mm}$ coverage at $300 \mathrm{~mm}$ was chosen as it was capable of being shone through the vacuum chamber viewport opposite to the microscope viewport and illuminate the meniscus for clear observation. The lighting setup shown in Figure (A.5) was mounted to the optical breadboard affixed to the rotary table using two mounting posts with a horizontal spacer beam in between to align it with the viewport. The light path between the source and the microscope objective was adjusted to get a clear screen image from the microscope imager.

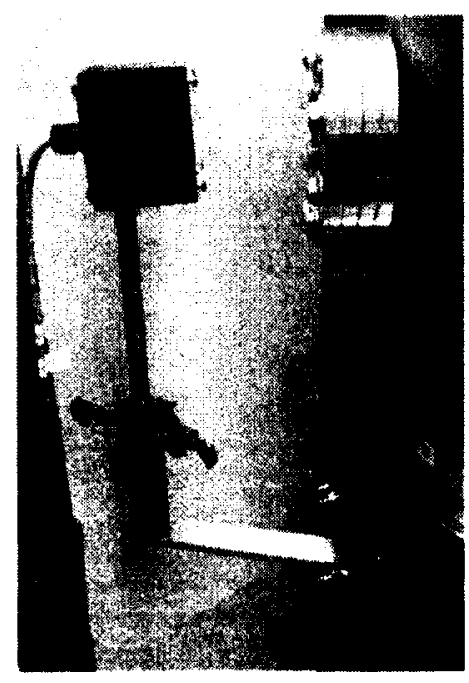

Figure (A.5): LED spotlight for chamber illumination through back window opposite of microscope 


\section{A.2.6 Data acquisition}

The non-imaging automated data acquisition was done using a Keithley 2700 multimeter switch system. Temperature, heater input voltage, pressure transducer voltage, and resistance for event recording of single pole single throw (SPST) switches were measured using a 40 channel multiplexer card (Keithley 7708).

\section{Temperature}

Thermocouples were used for temperature measurements because of their smaller size and faster response time in comparison to resistance temperature detectors and thermistors.

The recorded voltage difference at the thermocouple leads was internally converted to a temperature with a resolution of $\pm 0.001{ }^{\circ} \mathrm{C}$. A polynomial fit for T-type thermocouples was used to convert voltage to temperature. Automatic cold junction compensation was used to obtain an absolute temperature instead of a temperature difference [48], [53].

All thermocouples used in testing were of T-type with sub-miniature connectors and had $0.5^{\circ} \mathrm{C}$ special limit of error. The 'special' limit of error was related to the quality of the wire used and can be considered as the bias uncertainty of the wire [66], but not the overall bias uncertainty on the temperature measurement given the additional uncertainties for voltage measurement, voltage to temperature conversion, and cold junction compensation. The measurement of chamber ambient and early thick tube temperature testing was performed using $0.25 \mathrm{~mm}$ diameter (30 AWG) thermocouples with glass braid insulation (Omega 5SRTC-GG-T-30-36). Thermocouples used in later testing with small tubes were of 0.08 mm diameter (40 AWG) wire with Teflon insulation also from Omega (5TC-TT-T-40-36). 


\section{Vapour chamber pressure}

Chamber vapour pressure was measured using a pressure transducer from Omega (PX429$015 \mathrm{~A} 5 \mathrm{~V}$ ) with a range of $0-103 \mathrm{kPa}_{a}$. A signal between $0-5 \mathrm{~V}$ was recorded and converted to a pressure reading using a linear calibration curve. The transducer used a piezoresistive sensor with a total accuracy of linearity, hysteresis and repeatability of $\pm 0.08 \%$ of best straight line curve fit.

\section{Event recording}

A switch system was devised to mark significant events during testing. This was done by wiring a SPST switch in parallel with a resistor. The wire and switch resistance in parallel resultant reading was recorded with the switch on and only the relatively high inserted resistor was measured with the switch off. The switch was manually flipped when the onset of oscillation was noticed on the microscope display screen. Switching between all measurements within the Data Acquisition System (DAQ) was performed internally using a latching mechanical relay system. The software used to display the signals measured by the DAQ was: "Integra up and running with testpoint", version B03.

\section{Digital microscope image recorder}

In order to capture the meniscus behaviour throughout testing, a digital microscope imager (Celestron 44421) having a 2 megapixel sensor and a 15X magnification lens was used. Having two distinct magnifications for the eyepieces gave the advantage of using the 10X magnification reticle for big picture observation and not getting 'lost' when looking at the meniscus, while the $15 \mathrm{X}$ magnification imager could zoom into the meniscus in more detail. The software package (Digital Microscope Suite 2.0) was used to capture video in Windows Media format with up to 800 by 600 pixel resolution. A 640 by 480 
pixel resolution was chosen for video capture due to the smoother real-time playback. The video was displayed on a computer screen measuring $345 \mathrm{~mm}$ by $195 \mathrm{~mm}$ (Dell Inspiron 1545, Intel Pentium Dual CPU T3400 at $2.17 \mathrm{GHz}, 4.00$ GB RAM, Windows Vista SP2 32 bit). A foreground reticle shown in Figure (A.6) made from a transparency with vertical graduations every $2 \mathrm{~mm}$ was placed on top of the screen for reference.

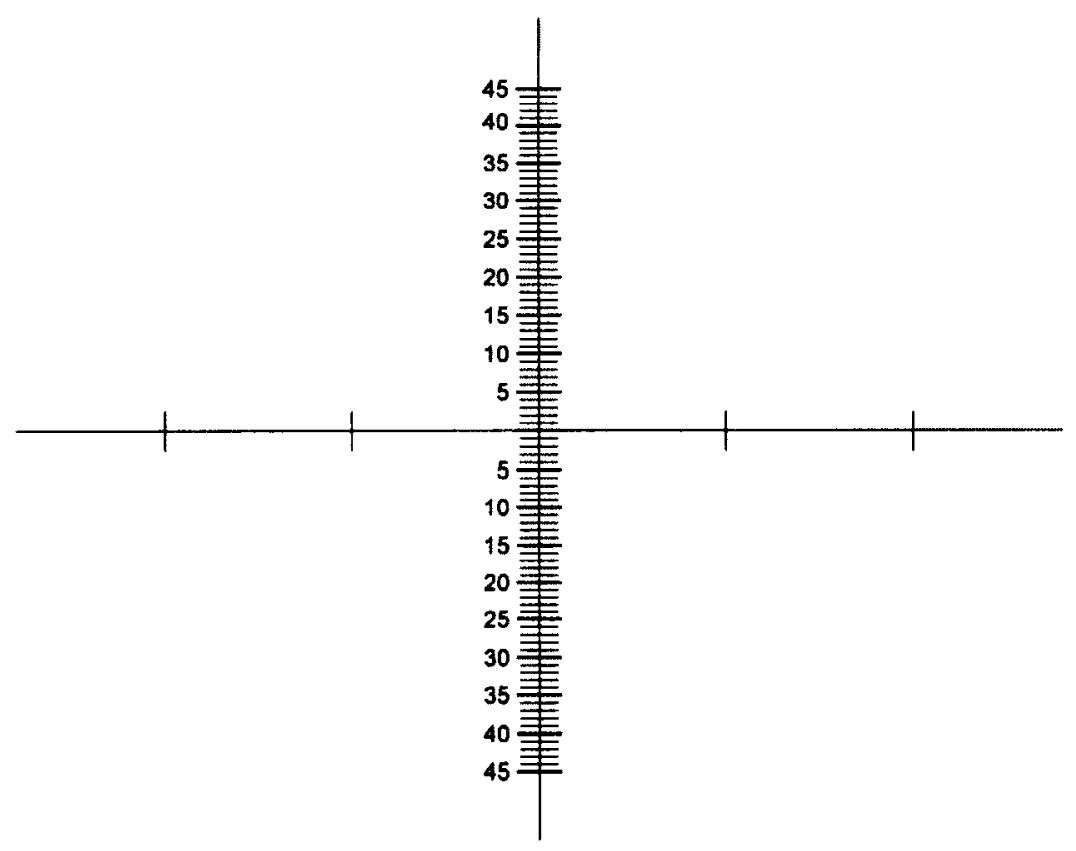

Figure (A.6): Screen display reticle

This allowed for close monitoring and adjustments to the reservoir as the meniscus level dropped. The reservoir was raised as the meniscus fell due to the decrease in surface tension and evaporation. The meniscus position was adjusted such that deviation from its reference location after adjustment was less than $0.01 \mathrm{~mm}$ for the $0.5 \mathrm{~mm}$ ID tubes and less than 0.02 $\mathrm{mm}$ for the $1.0 \mathrm{~mm}$ and $1.2 \mathrm{~mm}$ ID tubes. The meniscus level was allowed to drop less than $0.1 \mathrm{~mm}$ for the $0.5 \mathrm{~mm}$ ID tubes and less than $0.2 \mathrm{~mm}$ for the $1.0 \mathrm{~mm}$ and $1.2 \mathrm{~mm}$ ID tubes. This drop range was chosen as it was well within the camera field of view and since continuous manual adjustment would be impractical. It is also noteworthy that reservoir adjustment right before the onset of oscillations did not follow a strictly linear relationship 
between the rise in the meniscus and reservoir height as it would remain hinged to the wall requiring more technique and forethought to place the meniscus at the reference location between the thermocouples. Audio was also recorded within the same file as the video and notes were made when the power and reservoir settings were varied. Anomalies were noted to avoid confusion for later reference.

\section{A.2.7 Static feedthroughs}

\section{Heater leads}

Current to the tube heaters was provided by a laboratory DC power supply (GW Instek GPS-1830D) providing between $0-18 \mathrm{~V}$ up to a $3 \mathrm{~A}$ load. Voltage was controlled using a coarse and fine rotary dial. Amperage was displayed on the same screen as voltage and recorded for every power increment. The power supply voltage was tapped in parallel for recording using the DAQ. Two power leads were fed into the chamber through a $Q F-40$ wall feedthrough. A female DB-9 connector was attached to the feedthrough inside the chamber. Connection and disconnection with a male DB-9 plug shown in Figure (A.7) fixed to the baseplate optical breadboard was made with the bell jar just overhead of the baseplate allowing for manual socket access. Wire leads terminating with male crimp connectors were soldered to ends of the heater leads. Wire leads terminating with female crimp connectors were soldered to the DB-9 terminal on the baseplate for ease of assembly and disassembly. The additional resistance measured across a crimped lead was negligible compared to the typical heater resistance to not warrant soldering the entire circuit and losing the versatility of plug and socket connections. 


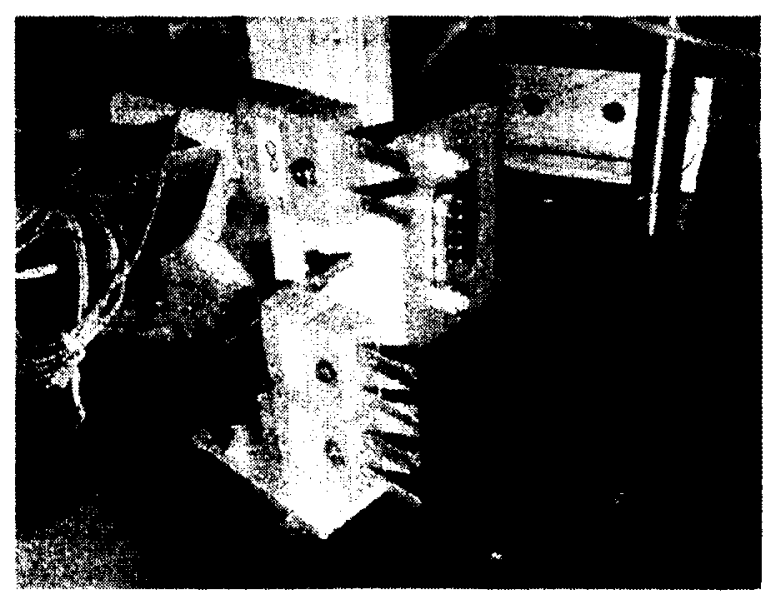

Figure (A.7): Power and thermocouple plugs inside of chamber for connection before closing the chamber and disconnection after opening the chamber

\section{Temperature and pressure}

Thermocouples for measuring tube and ambient chamber temperatures were connected to a QF-40 chamber feedthrough using the subminiature connectors shown in Figure (A.7). The vapour chamber pressure feedthrough was located at the top of the bell jar and utilised a QF-40 connection.

\section{A.3 Positioning subsystems}

\section{A.3.1 Vertical motion of the bell jar using a crane}

In order to safely and accurately mate and remove the stainless steel bell jar from the baseplate, a manually operated hydraulic crane with a geared cable winch was utilised as shown in Figure (A.8). The crane was bolted to the steel frame which supported the entire setup. Although the crane had a cable stop mechanism to allow for suspension of the bell jar directly over the baseplate for feedthrough connection and disconnection, the risk of the bell jar collapsing onto the hands of the person making the connections in the event 


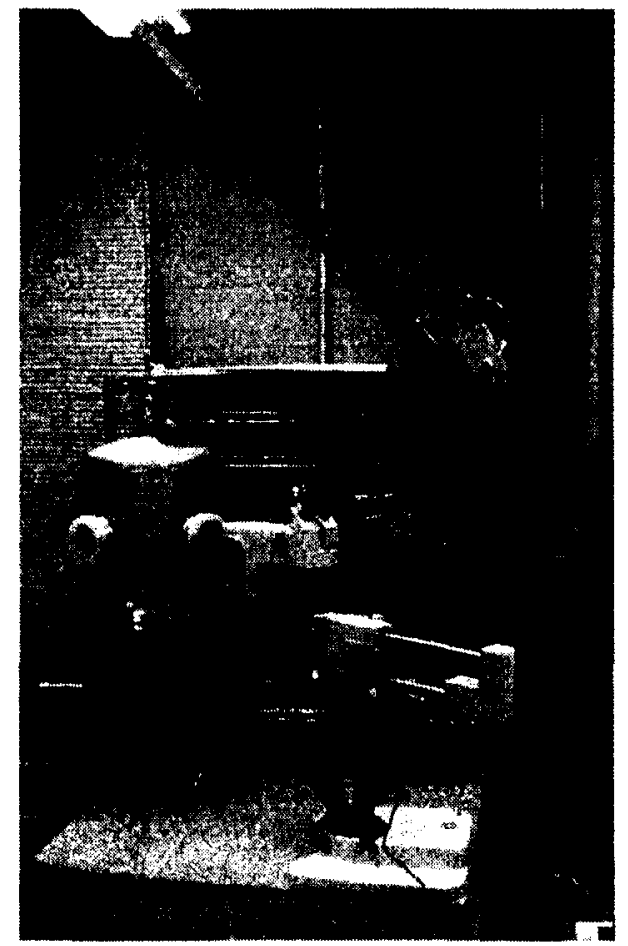

Figure (A.8): Bell jar moving crane

of winch failure or cable slip was considered unacceptable. This risk was mitigated by the placement of three wooden blocks around the circumference of the baseplate shown in Figure (A.9) to act as spacers in the event of a collapse.

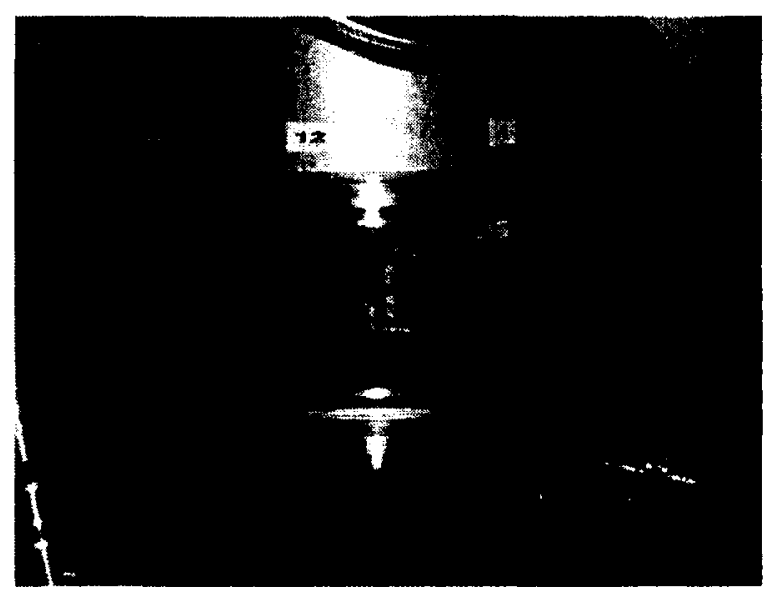

Figure (A.9): Safety spacers for connecting and disconnecting feedthroughs from chamber baseplate to bell jar 


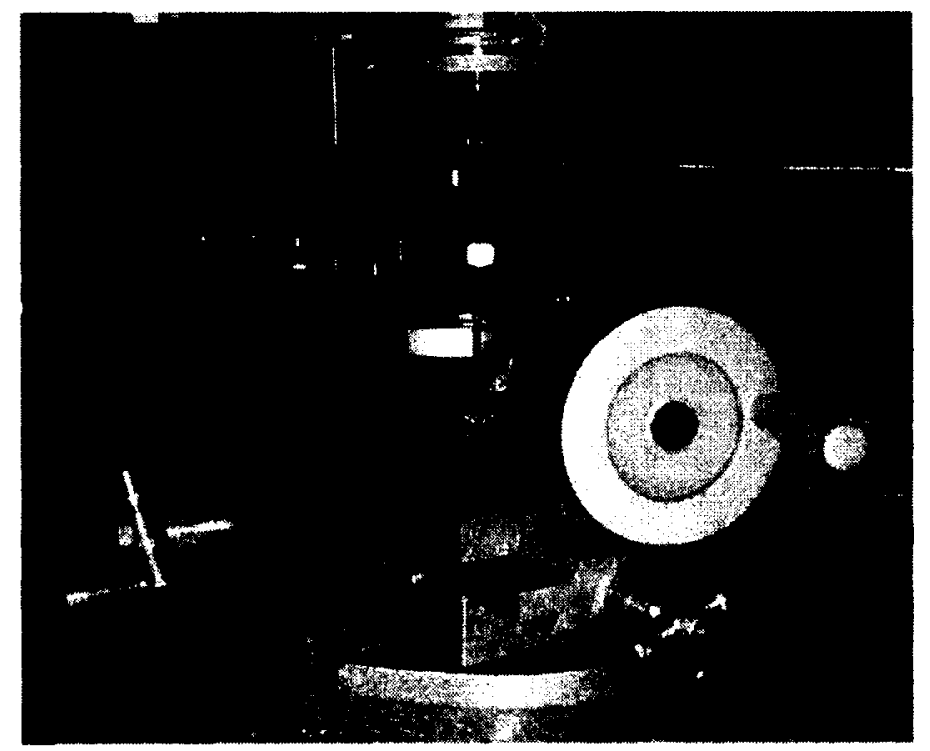

Figure (A.10): Translation and rotation system

\section{A.3.2 Translation and rotation of chamber}

In order to position the capillary tube in view of the microscope objective, a means by which to move the entire setup with accuracy and precision was required. The solution provided in this study is shown in Figure (A.10). A compound slide (Busy Bee 339C) with leadscrews having 10 threads per inch and maximum travel length of $110 \mathrm{~mm}$ in the direction of the microscope light path and $165 \mathrm{~mm}$ in the normal direction rested on the granite base and was used to move the setup linearly. A rotary table (Busy Bee B061) with a worm gear ratio of $90: 1$ and a base diameter of $195 \mathrm{~mm}$ was mounted on top of the compound slide to align the meniscus view port with the microscope objective.

Since the compound slide base was not secured to the granite table other than by friction and gravity, a counter moment was manually provided when tightening and loosening the chamber clamps to prevent the system from rotating.

Friction between the granite block and compound slide was found to be sufficient for the setup not to slip during the applied loads (valve operation and manipulation of the rotary drive) during testing. 


\section{A.3.3 Reservoir}

The reservoir used for testing shown in Figure (A.20) was a cylindrical crystallising dish (Pyrex 3140) with a $100 \mathrm{~mm}$ diameter and $50 \mathrm{~mm}$ height. The measured reservoir volume was $355 \mathrm{ml} \pm 2.5 \mathrm{ml}$. A crystallising dish was used since it has no spout under its rim which would otherwise reduce the maximum fill height to below the top edge of the reservoir and potentially block a low laying meniscus from observation. The reservoir was held in place to a bracket fixed to the linear slide within the chamber using a stainless steel worm gear clamp wrapped around two posts bolted to the bracket diametrically opposed across the reservoir.

A cover was placed on the reservoir for ambient testing in the acrylic chamber to reduce the evaporation rate. A cover was not used in vacuum testing because the extent of evaporation was reduced as saturation pressure was approached. Gross evaporation from the smaller reservoir would still on average condense more on the larger chamber surface area thereby lowering the reservoir level within the time scale of testing. Having the feed line outside of the reservoir for vacuum testing also made use of a cover more cumbersome and would require some means of funnelling the liquid into the reservoir to reduce spillage associated with filling a small opening from a distance. The funnel would also have to positioned over the reservoir such that it would not obstruct the illumination light path. This funnelling setup was fabricated for later use, but not used in stability testing presented in this work. It was observed during informal testing that the ceiling of a cover placed over the liquid reservoir would act as a condensation site. When condensate formed a droplet with a weight exceeding surface tension forces, it would fall and disturb the reservoir liquid surface and corresponding meniscus position. 


\section{A.3.4 Meniscus positioning}

The need to have the meniscus at a constant position throughout the test was important for the following three reasons. Firstly, it was important to measure the temperature difference between two fixed thermocouples to obtain a consistent temperature gradient throughout the test. Secondly, the proximity of the meniscus to the heater was critical in triggering instabilities in a reproducible manner since they would not form if the meniscus was far enough away from the heater, but would form with the meniscus closer to the heater for a given heat input. This was found to be the case especially in ambient testing where a higher temperature gradient was required to trip oscillatory behaviour. Finally, keeping the meniscus in the same position allowed for video capture without having to undergo the difficulty of moving the microscope during testing.

Movement of the meniscus in the axial direction during testing was due to decreasing surface tension with increasing temperature and evaporation from the reservoir. Evaporation of n-pentane from the reservoir was seen to be much more prevalent in testing with ambient air compared to a closed n-pentane environment. This effect was affirmed by the measurement of the meniscus height initially before heat was applied, and after the heating cycle was applied and the system returned to ambient temperature. The drop of the meniscus as a function of applied heater voltage is shown in Figure (A.11). Each height recording was taken after having moved the reservoir such that the meniscus was at its reference position between the thermocouples on the outside of capillary tube. Voltage was applied using steps and the square data points in Figure (A.11) refer to the adjustment at the time right after the meniscus dropped after a voltage increase. After this reading, additional time was allotted to observe the meniscus behaviour and verify its temperature stability. Once stability was confirmed, the meniscus height was adjusted and recorded to account for evaporation and temperature rise before raising the voltage to the next step. After reaching the 


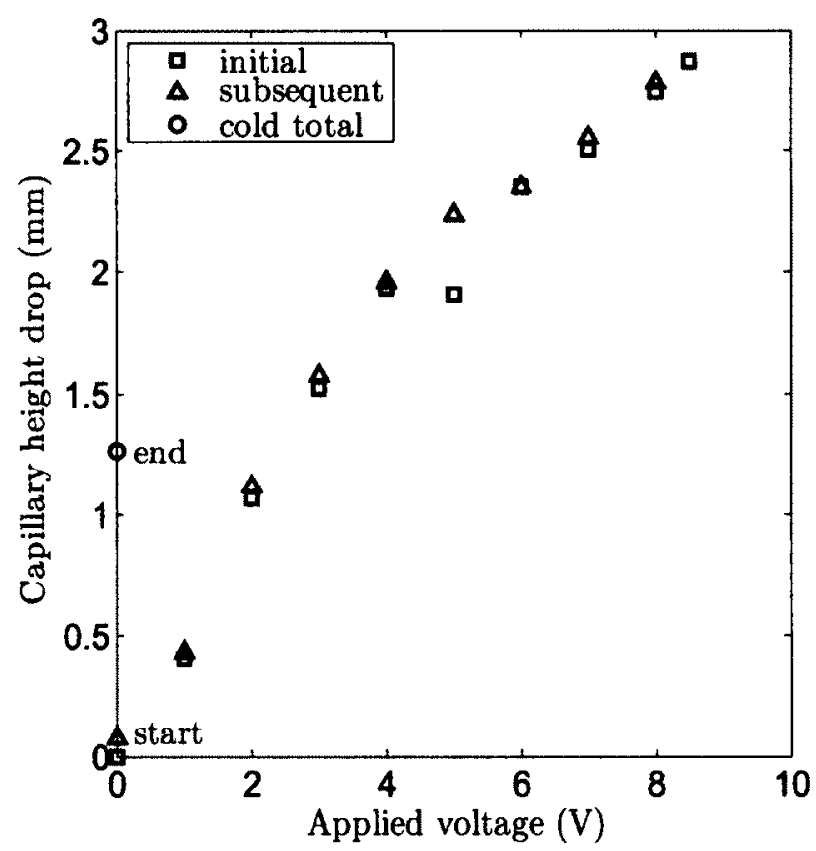

Figure (A.11): Meniscus height drop with voltage for ambient testing of $1 \mathrm{~mm}$ ID thin tube where the points at the same voltage represent different times for that given voltage. After voltage is applied and meniscus falls; (square) and before raising voltage to the next step; (triangle) for that given voltage setting. The total drop at the test end after cooling to ambient; (circle).

maximum voltage, power was shut off and time was alloted for the meniscus to return to ambient temperature before taking the final height reading. It is of interest to note that at the $5 \mathrm{~V}$ setting for the case shown in Figure (A.11), the meniscus actually rises compared to the previous lower voltage setting. This could be attributed to high evaporative cooling lowering the interface temperature, resulting in a higher surface tension. At the $6 \mathrm{~V}$ setting, it was found that the meniscus would not move, regardless of the increasing temperature. This behaviour was observed consistently with different tests. Observing Figure (A.11), one can see the meniscus drops nearly three times the inner tube diameter and on the order of the typical thermocouple spacing distance. If one were to keep the tube stationary, this would necessitate a larger field of view and corresponding lower magnification to observe 
the meniscus continuously using a stationary microscope. Alternatively, a positioning system for the microscope would be required to track the meniscus as it moves to still be able to monitor its behaviour. Also, the thermocouple spacing would have to be enlarged or multiple thermocouples would have to be placed on the tube to measure a gradient across the meniscus if the reservoir was not adjusted. Finally, power applied to the heater would have to be increased to obtain the same temperature profile near the meniscus due to the axial temperature drop along the tube between the heater and migrating meniscus.

The decreasing capillary height with increasing heat input was due mainly to the surface tension decrement with temperature. This was apparent when heat input was discontinued after destabilising the meniscus, resulting in a rising meniscus as is shown when comparing the last two points in time in Figure (A.11).

A greater drop in reservoir height was observed when testing with the thick versus thin tubes due to more reservoir evaporation since more time was needed to reach thermal equilibrium as a result of the greater thick tube thermal capacity.

To compensate for the dropping meniscus during testing, the reservoir position was changed by turning a rotary feedthrough connected to a linear stage and measured using a deflectometer as shown and labeled in Figure (A.12)

\section{Rotary feedthrough}

A manually driven rotary feedthrough allowed for a shaft to rotate through the chamber floor. The feedthrough (Magidrive MD16) used magnetic coupling to provide up to 0.45 $\mathrm{Nm}$ of torque with no backlash detected under low loading out of a shaft with an M5 female thread. The mechanism that translated rotary motion to linear motion in order to move the reservoir is shown in Figure (A.13). The rotary feedthrough shaft was fitted to the slotted rotating shaft coupling that connected with the linear stage. 


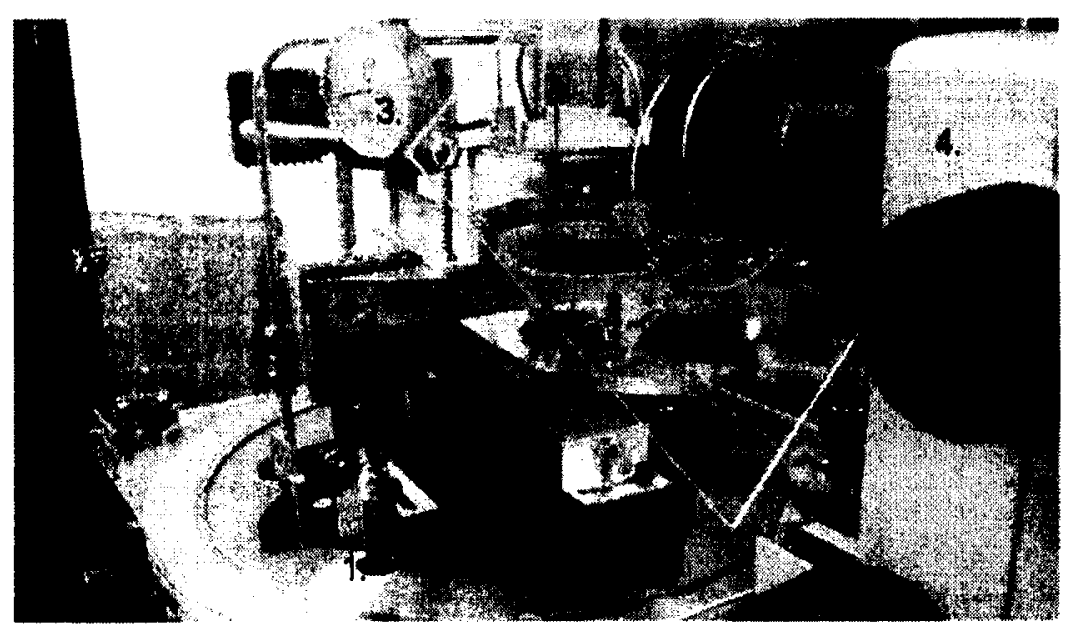

Figure (A.12): Reservoir position control and measurement in ambient: 1. rotary feedthrough, 2. linear stage, 3. deflectometer, 4. microscope

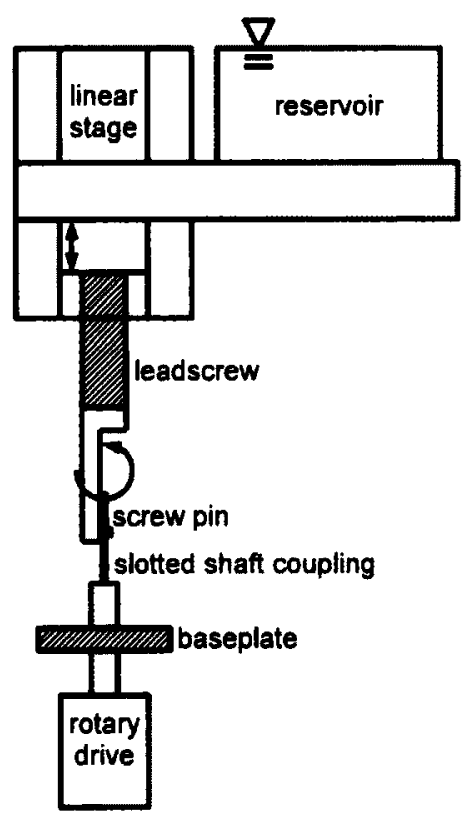

Figure (A.13): Rotary mechanism to move reservoir 


\section{Linear stage}

The linear stage (Newport M-UMR5.16) was moved by rotating a leadscrew terminating with a ball bearing point pushing a carriage which slid along bed ways fixed with respect to the chamber base. The carriage was constrained by precision double-row ball bearings on either side in contact with the bed ways. Backlash was eliminated by two springs providing tension between the bed and carriage. The maximum travel was $16 \mathrm{~mm}$ with a maximum vertical load capacity of $40 \mathrm{~N}$.

The slide leadscrew was connected to the rotary feedthrough using a slotted rotating shaft. A screw pin was mounted to the leadscrew normal to its axis of symmetry with the freedom to move vertically while being confined to move angularly with the slotted shaft. Backlash was reduced by tightening the screw pin to reduce the play between the slotted shaft and leadscrew. As a result, backlash was not sensed in the form of a time delay when manually rotating the rotary shaft and observing movement of the deflectometer needle.

\section{Deflectometer}

The vertical position of the reservoir with respect to its support frame was measured using a dial deflectometer with graduations every $0.0254 \mathrm{~mm}(0.001$ inch). The gage was mounted to a beam fixed to a post on the chamber baseplate and the moving core was positioned normal and touching the reservoir base. The gage was positioned such that it could be read through the side viewport of the bell jar throughout testing.

\section{A.3.5 Scale calibration}

A process was devised to find the actual magnification factor when looking at the meniscus image as it travels through the chamber, microscope, camera, and finally appears on the computer screen. This was done by moving the meniscus without applying heat and when 


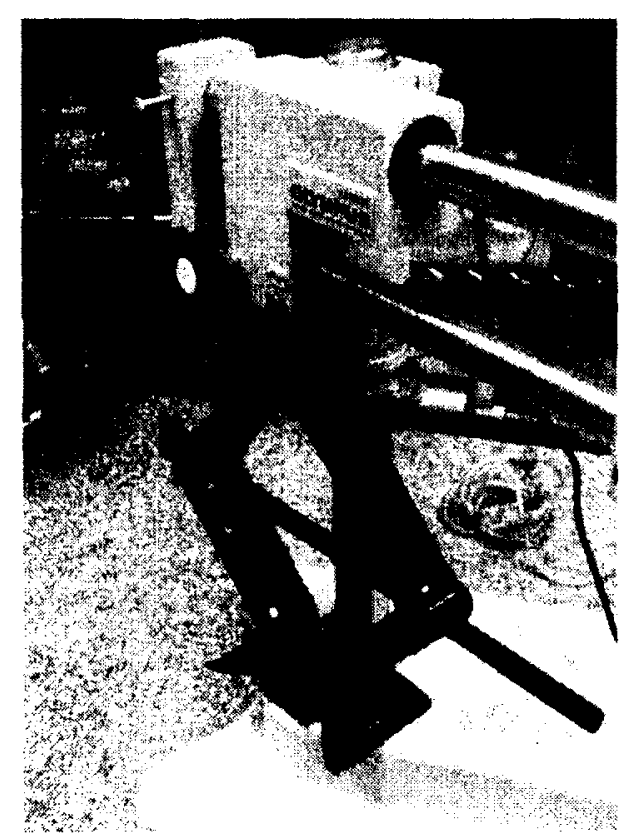

Figure (A.14): Screw jack microscope positioning mechanism

temperature was steady. The meniscus was brought to a reticle marking near the top and bottom of the screen and the corresponding deffectometer readings were noted. The ratio of the changes in those two readings are taken as the scaling factor.

\section{A.3.6 Microscope positioning}

The stereo research microscope used during testing was attached to a support stand (Olympus SZ-STU2) allowing for vertical, horizontal, and rotational adjustment to capture the meniscus. The rotational and horizontal adjustments were adequate for minute adjustments, but vertical adjustments could not be made at the level required to see the meniscus due to the awkwardness of having to support the microscope weight. A scissor screw jack was used to support the microscope weight and the fine thread of jack screw allowed for adequate height control. To prevent rotational motion while the microscope was being moved vertically, the post friction clamp was partially engaged while still allowing for vertical motion. The setup is shown in Figure (A.14). 


\section{A.4 Fluid control}

\section{A.4.1 Chamber sealing}

Creating a tight seal between the bell jar and baseplate was accomplished by using a Viton o-ring held in place with a retaining ring recessed inside a groove within the chamber baseplate. Vacuum grease was initially applied, but the practice was later rejected as it is typically recommended only for glass and moving seals [67]. Firstly, ubiquitous silicone grease could not be used to chemical incompatibility with n-pentane. A suitable hydrocarbon based grease (Apiezon 101) was applied, but testing showed no noticeable increase in vacuum longevity when using a greased seal. The grease also would dry up after a number of tests and made cleanliness more difficult to maintain within the chamber giving reason more for the abandonment.

The o-ring was compressed between the bell jar flange and baseplate with a series of 12 double claw aluminium clamps bolted around the circumference of the chamber. By numbering the bell jar from 1-12 every $30^{\circ}$ with corresponding clamps, the following bolt tightening pattern was observed: $12,6,3,9,1,7,4,10,2,8,11,5$. A torque wrench was used for the bolt sequence and a relationship between the bolt torque and pressure loss after 15 minutes was determined. Figure (A.15) shows the expected relationship of a decrease in pressure gain as the bolt torque is increased. The change in $\Delta P$ decreases with increasing bolt torque indicating an insignificant benefit from tightening the bolts beyond the maximum applied torque. Tightening the bolts near the maximum value would warrant either fixing the entire test setup to the steel box supporting the granite block or the granite block itself since friction and manual torque counteraction does not keep the setup stationary. 


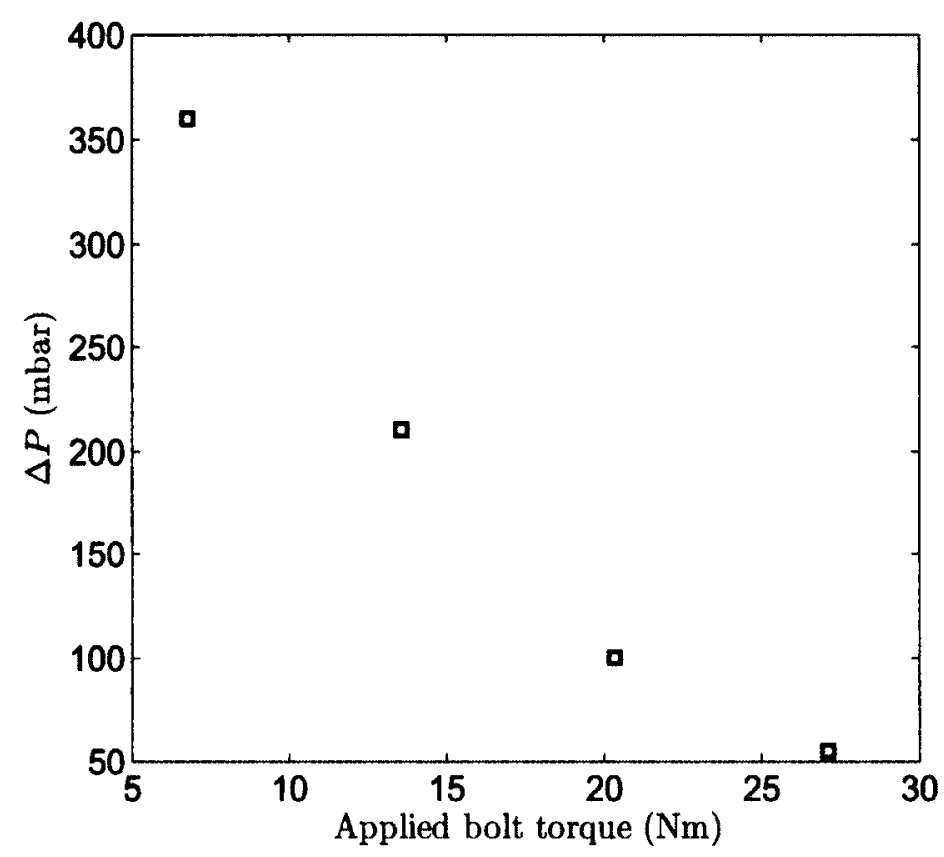

Figure (A.15): Evacuated chamber pressure increase with applied torque after 15 minutes

\section{A.4.2 Chamber evacuation}

The system developed to evacuate the vacuum chamber is shown in Figure (A.16). Removing air from the chamber was performed using a two stage rotary vacuum pump (Alcatel Pascal 2005 SD) capable of reaching an absolute pressure of $2 \times 10^{-3}$ mbar. Upstream of the pump was a pressure gage, copper mesh filter, purge valve, and finally a right angle valve with a QF-25 fitting connected to the baseplate of the vacuum chamber. A length of wire reinforced flexible tubing was used to connect the purge valve and baseplate valve to allow for movement of the chamber assembly for positioning with respect to the microscope. Evacuation was performed with the chamber being dry due to the difficulty in pumping liquid with a vacuum pump and the general health and safety concerns involved in exhausting $\mathrm{n}$ pentane vapour. Additionally, if liquid remained in the reservoir from a previous test before evacuation, the vacuum pump was not able to remove it entirely and vapour bubbles would then be trapped and resurface during testing. The chamber was evacuated to remove the 


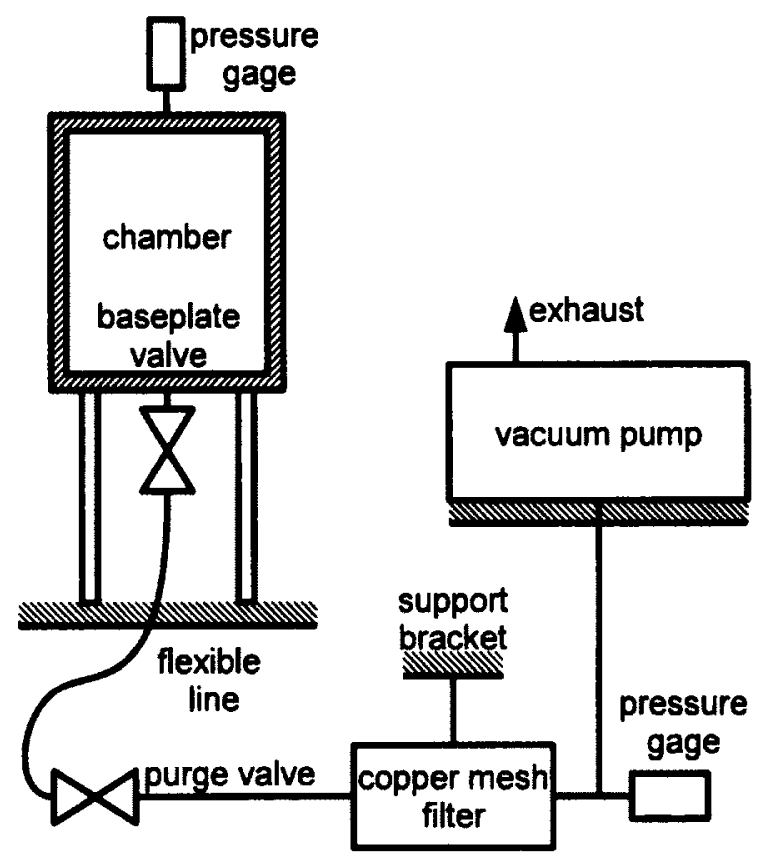

Figure (A.16): Vacuum plumbing system

great majority of ambient air in order to have a nearly pure n-pentane environment that can be considered pure for the purposes of this study. Evacuating to the capacity of the pump would be a waste of time. The evacuation process chosen would take around 15 minutes and was considered adequate when the pressure transducer on the bell jar registered zero absolute pressure as viewed on the Keithley multimeter display. The vacuum pump run time was also chosen such that it would get sufficiently warm that water in the air being evacuated was less likely to condense inside the pump after it was shut off compared to with a cold shutdown.

The number of removable joints and valves used in the test system warranted a leak test to assure chamber conditions would not change significantly during a test and complicate results with a time dependent component. To evaluate the rate at which the chamber leaked, the chamber vapour pressure once evacuated and sealed was monitored for a period of time over five times greater than a typical test series. The leak test consisted of evacuating the sealed chamber with the needle flow control valve open. Immediately after closing the 


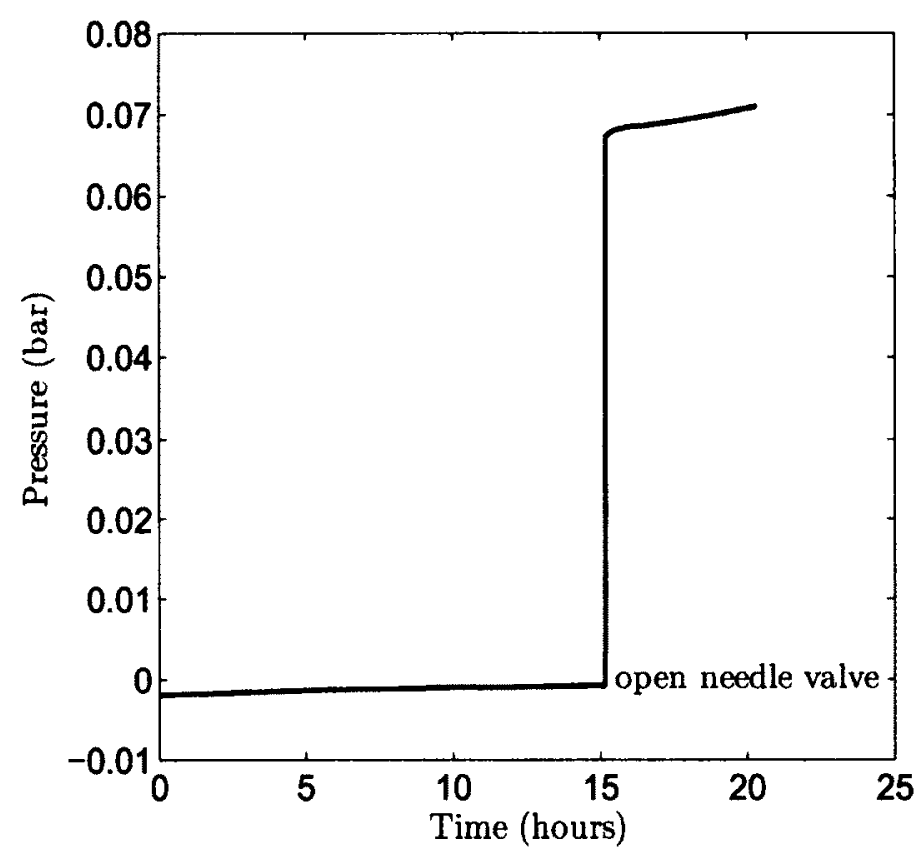

Figure (A.17): Measured chamber pressure over extended time with needle valve opening gas evacuation valve, the liquid needle valve was closed and vacuum pump was shut off. The system was left to sit for $\mathbf{1 5}$ hours. Afterwards, the needle valve was opened and a pressure jump was observed as noted in Figure (A.17). The jump in pressure when the valve was opened clearly indicates the liquid fill system leaked. Also, the slope for the pressure versus time curve is more than double in the portion with the needle valve open compared to closed, indicating the leak rate in the liquid feed line is greater than that of the chamber alone. The results described above indicate that leaking did play a role in the testing process. Practically, it means that the chamber could not be evacuated and left idle sitting before testing without affecting the results. This became evident when performing a test on a given day, leaving the chamber filled with n-pentane overnight and repeating it the following day only to consistently yield different results. The results differed such that the temperature gradient corresponding to the onset of oscillations would increase when a test was performed the next day. This is in line with the limiting case of testing in ambient air 
where the temperature gradients were much greater than in a vacuum environment. It was found that when performing three identical tests in series without delay that the results were independent of the test order. Testing was performed with two types of tubes with similar internal diameters, but thin tubes having a wall thickness of $0.1 \mathrm{~mm}$ and thick tubes having an average wall thickness of $2.3 \mathrm{~mm}$. It was then concluded that the physics contributing to meniscal destabilisation are a weak function of the change in ambient chamber vapour pressure and fluid composition for the roughly three hour period it takes to run three tests for a thick tube. The test time period was less for thin tubes since they reached steady state faster after a change in heat input due to their lower heat capacitance. Having a perfectly sealed chamber would not mean that a testing environment could be sustained indefinitely due to the surface evaporation within the reservoir and condensation at the bottom of the chamber that occurs over time. Also, the embrittlement of the wire sheathing from long term exposure to n-pentane vapour warrants prompt testing once the chamber is charged and evacuation after test completion.

\section{A.4.3 Liquid manipulation before and after testing}

\section{Before}

Approximately $400 \mathrm{~mL}$ of liquid n-pentane was poured from a $4 \mathrm{~L}$ jug into a $1 \mathrm{~L}$ graduated cylinder with markings every $10 \mathrm{~mL}$. The cylinder height was roughly $46 \mathrm{~cm}$ and this allowed for accurate pouring without spillage compared to a wider and shorter beaker due to the greater tilt control with the taller beaker. Unwanted evaporation from the cylinder was also reduced by having a tall beaker as it reduced the surface area for evaporation. The taller beaker height also reduced liquid loss by lowering diffusion as the concentration gradient from the liquid surface to the beaker exit is less for a taller beaker. An aluminium foil cap was wrapped around the mouth of the beaker to further reduce evaporation and 
contamination for external sources.

\section{After}

Liquid n-pentane was drained out of the chamber after testing via two methods. After the chamber pressure was equalised with ambient and the bell jar was removed, a syringe with Tygon tubing fitted to the end was placed inside the internal reservoir to syphon the majority of the n-pentane. Some liquid remained inside the internal reservoir due to the beads obstructing the flow and breaking the continuum necessary to maintain a syphon when draining near the bottom of the reservoir. The interface where the liquid, ambient air, and beads met had a low evaporation rate compared to the interface of liquid with ambient air. To accelerate the evaporation process, heat was applied to the base of the reservoir using a laboratory hot plate or thermoelectric cooler with reversed polarity to evacuate the remaining n-pentane so that it would not impede the evacuation process in subsequent testing. Liquid left inside the external reservoir and fill line was drained from a port at the lowest point of the drain line. Used test fluid was not reused in order to avoid contamination in future tests and to maintain a level of consistency.

\section{A.4.4 Liquid delivery to reservoir for vacuum testing}

The procedure involved in filling the internal liquid reservoir to where the meniscus resides between two thermocouples on a capillary tube is described below. The plumbing system for transferring liquid into the chamber is shown in Figure (A.18) where the semicircles in the diagram represent compression fittings. The process began after the chamber had been evacuated and the baseplate vacuum valve was closed. An external stainless steel liquid reservoir open to ambient was mounted above the capillary tube outside of the vacuum chamber. Two valves were swaged in series along a stainless steel line with $6.4 \mathrm{~mm}(0.25$ 


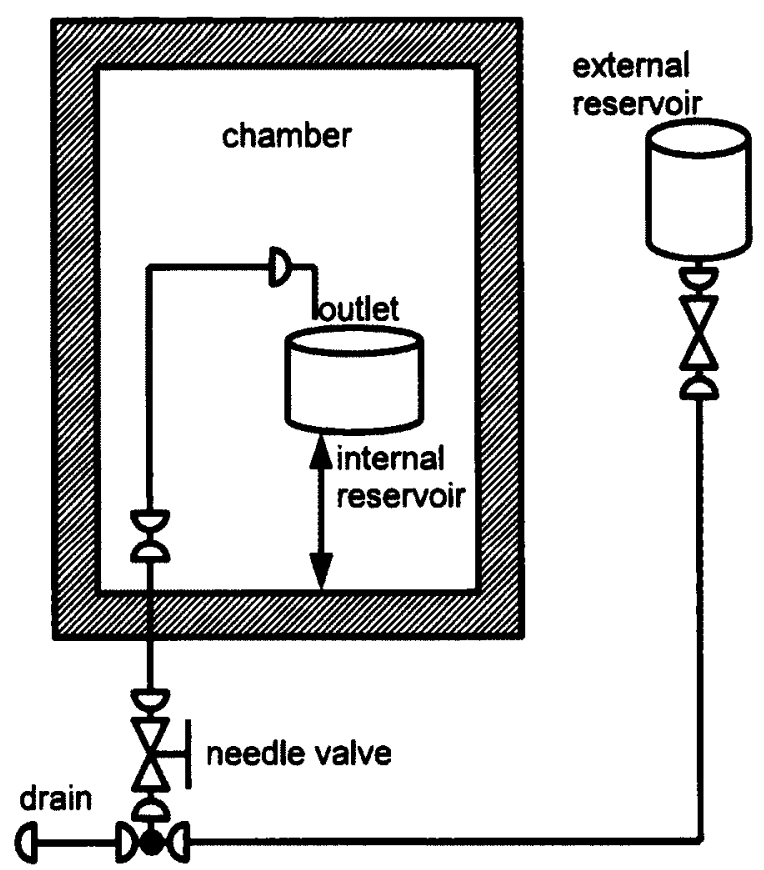

Figure (A.18): Liquid plumbing system

inch) outer diameter and $0.89 \mathrm{~mm}(0.035$ inch) thickness to control the flow being outlet to the internal reservoir. The first valve (Swagelok SS-4BK) was meant to isolate the liquid line from the atmosphere during purging by being fully closed and then being fully opened after filling the external reservoir with liquid. The valve was then closed once enough liquid entered the chamber such that the meniscus was at its desired position between the thermocouples with the reservoir at its lowest prescribed height. The reservoir was kept initially low so that it could be raised throughout testing to compensate for the liquid level drop due to evaporation. Given the coarseness of flow control via the first valve, a precision needle valve (Ideal Valve 54-1-11) with over 22 turns for flow control was placed downstream just before the chamber liquid feedthrough. The valve had a $0.79 \mathrm{~mm}(1 / 32$ inch) orifice, $1^{\circ} 21^{\prime}$ needle taper. Metering the flow was of high importance, especially when starting to fill the empty chamber. Due to the initial chamber pressure being lower than the vapour pressure, the fluid would enter as a gas. The gas entering the chamber would do so rather violently if the pressure within the line was not lowered significantly from its near ambient 


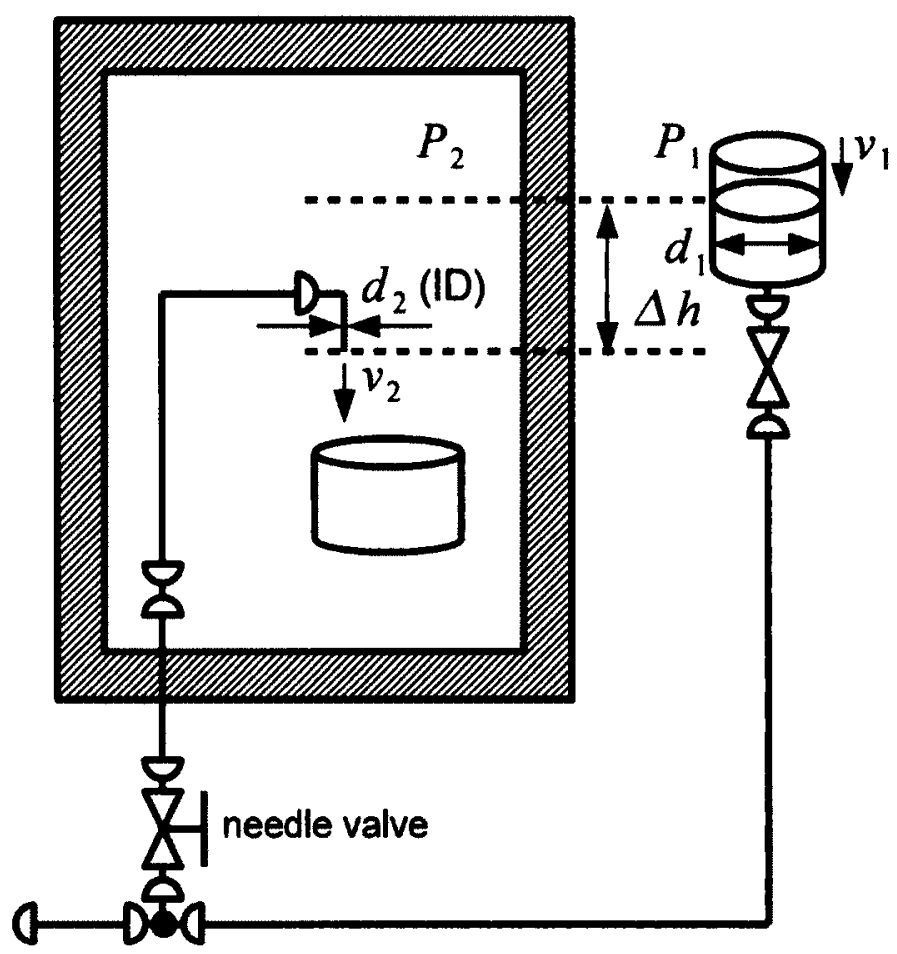

Figure (A.19): Parameters for obtaining an upper limit for the outlet velocity inside the vacuum chamber, $v_{2}$

state to near vacuum. Issues arising from a non-throttled outlet are the displacement of the vibration dampening glass spheres in the chamber, possible tube damage due to high velocity gas circulation. Also, once the chamber pressure reaches the vapour pressure, liquid splashing out of the reservoir would end up on the tube, viewports, or baseplate in a wasteful manner. Before metering the flow as described above, three pressure snubbers (Omega PS-4E) with $0.033 \mathrm{~mm}$ mean pore size were placed in series to provide a pressure drop. The lack of flow control with this method was deemed unsatisfactory. A temperature drop was noticed when filling the chamber with fluid as its expansion process is endothermic to the point where frost developed on the needle valve surface exterior. The temperature drop was not large enough to compromise the o-ring seals.

An upper limit for the outlet liquid velocity can be calculated by assuming frictionless flow and solving Bernoulli's equation for the conditions shown in Figure (A.19). We 
consider the chamber vapour pressure inside increasing from near zero to where the fluid exiting at (2) is liquid. The velocity is obtained by solving Equation (A.1):

$$
v_{2}=\sqrt{\frac{2\left(P_{1}-P_{2}+\rho g \Delta h\right)}{\rho\left[1-\left(\frac{d_{2}}{d_{1}}\right)^{4}\right]}}
$$

Table (A.1): Analysis summary for upper limit of liquid velocity entering internal reservoir for vacuum and ambient cases

\begin{tabular}{ccccccccc}
\hline \hline case & $\begin{array}{c}T \\
(\mathrm{~K})\end{array}$ & $\begin{array}{c}v_{2} \\
\left(\mathrm{~m} \mathrm{~s}^{-1}\right)\end{array}$ & $\begin{array}{c}P_{2} \\
(\mathrm{~Pa})\end{array}$ & $\begin{array}{c}\rho \\
\left(\mathrm{kg} \mathrm{m}^{-3}\right)\end{array}$ & $\begin{array}{c}d_{1} \\
(\mathrm{~mm})\end{array}$ & $\begin{array}{c}d_{2} \\
(\mathrm{~mm})\end{array}$ & $\begin{array}{c}\rho g \Delta h \\
(\mathrm{~Pa})\end{array}$ & $\begin{array}{c}P_{1}-P_{2} \\
(\mathrm{~Pa})\end{array}$ \\
\hline vacuum & 273.15 & 15.5 & 24453 & 646 & 65 & 4.62 & 317 & 76872 \\
vacuum & 300 & 9.6 & 73162 & 619 & 65 & 4.62 & 304 & 28163 \\
ambient & 300 & 1.0 & 101325 & 619 & 65 & 4.62 & 304 & 0 \\
\hline
\end{tabular}

The liquid density is assumed identical at (1) and (2), $P_{1}=101325 \mathrm{~Pa}$, and $\Delta \mathrm{h}=50 \mathrm{~mm}$. The parameters and results are shown in Table (A.1) where the temperatures analyzed are at the extrema bounding the actual ambient temperatures encountered during testing. From inspection, it is clear the exit velocity would be much too high considering the fragility of the test components without the aforementioned flow control valve for the cases with an ambient to vacuum pressure difference. It is also clear when comparing the hydrostatic pressure difference, $\rho g \Delta h$ and the ambient pressure difference, $P_{1}-P_{2}$ that the ambient pressure difference dominates by 2 orders of magnitude and so it would be impractical to reduce the liquid flow rate by having the reservoir below the chamber.

The filler line outlet inside the chamber was placed above the reservoir and not under the reservoir free surface because of consistent vapour pulses exiting the filler line due to the minute, yet detectable pressure difference between the feed line and chamber after both valves were closed. The filler tube was placed above and far enough away from the reservoir such that no motion was detected on the free surface when viewing the meniscus 


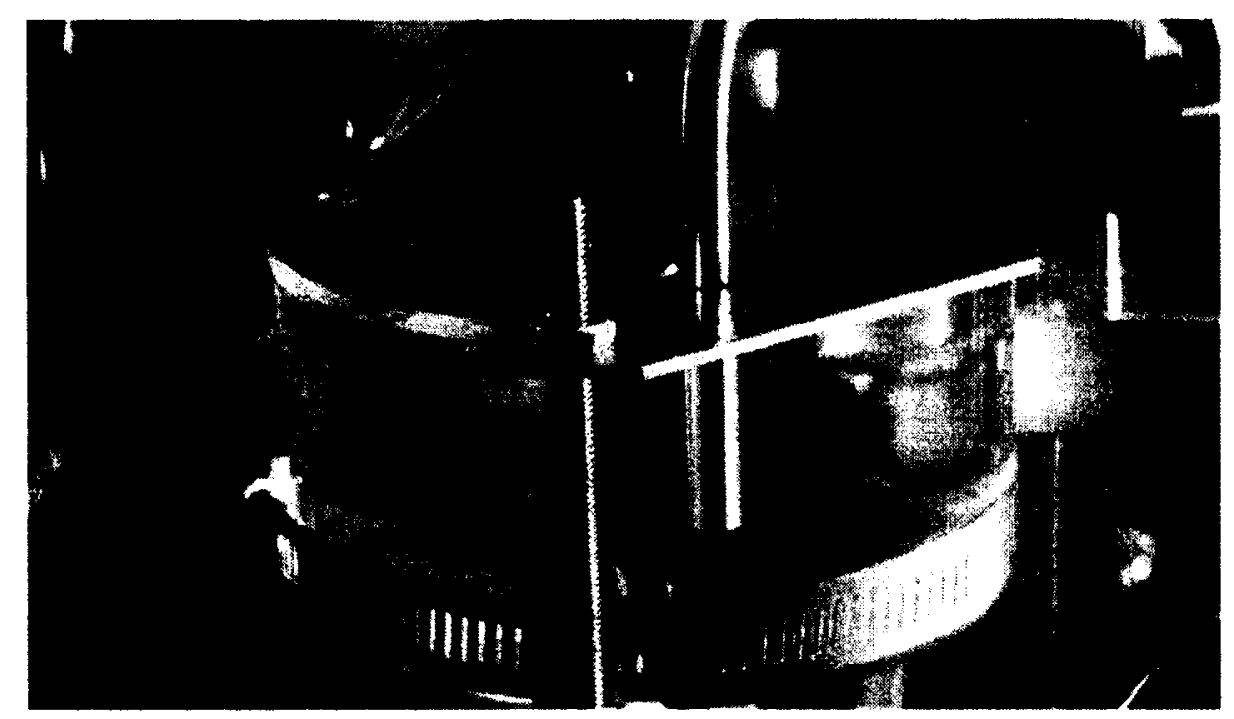

Figure (A.20): Reservoir under ambient testing

through the microscope. Upon emptying the liquid line after testing at the drain port located between the two closed valves, it was seen that the line was filled partially with gas due to the volume of liquid exiting being less that the line volume. This suggests incomplete priming of the liquid line when charging the chamber with fluid. Air inside the line as a result of leaking was ruled out since the pulse rate appeared too rapid to be caused by a leak which would otherwise have pressurised the chamber to ambient room pressure within the testing time period. The reason for incomplete priming is due to the length of the feed line and how suction from the low pressure vacuum chamber was used to charge the line, leading to two-phase flow in the feed line.

\section{A.4.5 Liquid delivery to reservoir for ambient testing}

The liquid delivery system for ambient testing was similar to that under vacuum with some notable exceptions partially shown in Figure (A.20). For instance, the outlet tube was submerged under the reservoir free surface. We note that no disturbances were observed under this condition compared to vacuum testing. Secondly, the reservoir was covered by a 
piece of sheet metal to decrease the evaporation rate and minimise contamination. Lastly, a metering valve was not required for flow control due to the flow being simply driven by gravity. Referring again to Table (A.1), the frictionless velocity limit for testing in ambient is somewhat high, but the tube wall friction, valve friction, and easily reducible $\Delta h$ make control of the ambient liquid velocity easily manageable.

\section{A.5 Capillary test tube manufacturing}

In order to observe the behaviour of an evaporating meniscus, a vessel small enough was needed so that capillary forces were significant, the surface in contact with the meniscus was quantifiable, heat could be applied to that surface, and the temperature about the meniscus could be experimentally determined.

Due to the novelty of this endeavour, a variety of methods were explored to contain the meniscus. Before arriving to the setup used described below, a variety of heated flat plates were placed in parallel to one another at varying distances using callipers, but testing in ambient did not destabilise the meniscus without boiling. It is thought that the lack of uniformity in heating a meniscus between parallel plates was cause for not capturing the phenomenon observed with the circular tubes. As a result, capillary tubes were used in testing.

\section{A.5.1 Stock thick and thin tubes}

Two sizes of thick tubes used were made of heat resistant borosilicate glass conforming to MIL-G-47033 specification with an inner diameters of $0.6 \mathrm{~mm}$ and $1.2 \mathrm{~mm}$ and outer diameters of $5.0 \mathrm{~mm}$ and $6.0 \mathrm{~mm}$, respectively. Stock lengths were longer than space constraints would allow, so they were cut to size by using a tubing cutter to score the surface and then snapped by hand. A consistent tube length of $40 \mathrm{~mm}$ was chosen for 
all test samples. The minimum length of the tube was such that it had to be long enough for the meniscus with the largest capillary rise to remain visible below the heater. The maximum height of a static meniscus can be determined by equating the capillary pressure at zero contact angle for a circular meniscus to the hydrostatic pressure difference between the meniscus and reservoir. This is shown for n-pentane in Equation (A.2a) where $\hbar$ is the height of the meniscus base above the reservoir

$$
\begin{aligned}
\mathscr{h} & =\frac{4 \sigma}{\rho g d} \\
& =\frac{4 \cdot 0.0177 \mathrm{~N} / \mathrm{m}}{641.3 \mathrm{~kg} / \mathrm{m}^{3} \cdot 9.81 \mathrm{~m} / \mathrm{s}^{2} \cdot 0.001 \mathrm{~m}} \\
& =22.5 \mathrm{~mm}
\end{aligned}
$$

and $\sigma, \rho, d$ are the surface tension, liquid density, and tube inner diameter, respectively. All properties were evaluated at $5{ }^{\circ} \mathrm{C}$ which is lower than any test temperature in this study, thus insuring a safe lower limit given that surface tension and therefore capillary height increase with decreasing temperature.

The upper limit on the tube height was imposed by the depth of the reservoir such that it could be raised with respect to the tube in order to bring the meniscus between the tube thermocouples without hitting the beads inside the reservoir. A hole in the beads was made under the tube by manually parting the surrounding beads allowing for more clearance.

The two thin tubes tested were made of quartz (Vitrocom T1012Q) with an inner diameters of $0.5 \mathrm{~mm}$ and $1.0 \mathrm{~mm}$, and outer diameters of $0.7 \mathrm{~mm}$ and $1.2 \mathrm{~mm}$, respectively. The inner diameter tolerance was $\pm 0.1 \mathrm{~mm}$. Similar to the thick tubes, lengths had to be cut to size. Due to the size and fragile nature of the thin tubes, scoring was performed by manually rotating the tube inside the collet of a pin vise while scoring it with a diamond file. A 
snap-cut was then performed, sometimes leaving jagged edges which were smoothed out with a diamond file.

\section{A.5.2 Thick tube thermocouples}

In order to capture the temperature gradient across the meniscus residing within a thick tube, the temperature was measured as close to the inner wall as practical to reduce thermal resistance. For this, blind holes were drilled into the tubes radially to insert temperature sensors. The process to drill into the glass was not trivial considering the size of the holes, hardness of the material, and external curvature of the tube. A glass tube drill rig shown in Figure (A.21) was devised consisting of a high speed drill press capable of reaching the supplier recommended 9000 RPM speed for the chosen drill bit. A $1 \mathrm{~mm}$ diameter multilayered electroplated diamond drill bit (UKAM 4ED10) was used to make the holes. The tube was held using a collet and fixed to a locking linear stage for accurate hole spacing. A pecking sequence was performed to drill the holes with water being added for both cooling and to create an abrasive colloidal mixture with the drilled out glass.

After the holes were drilled and carbon heater was deposited, the holes were cleaned out using acetone. A well of epoxy (J-B Weld) with a thermal conductivity of 0.590 $\mathrm{Wm}^{-1} \mathrm{~K}^{-1}$ [68] was created in the cavities and glass sheath insulated T-type $0.51 \mathrm{~mm}$ diameter lead thermocouples (Omega TC-GG-T-24-72) were inserted to their maximum penetration depth and left to cure. Before this thermocouple affixing method was chosen, thermal grease was used to lower the contact resistance, but it was seen too messy and mechanically unreliable for testing. The use of higher thermally conductive epoxy such as Omegabond 200 with a thermal conductivity of $1.38 \mathrm{Wm}^{-1} \mathrm{~K}^{-1}[66]$ was considered, but proved to be impractical as the cure temperature of $121^{\circ} \mathrm{C}$ for 8 hours would require 


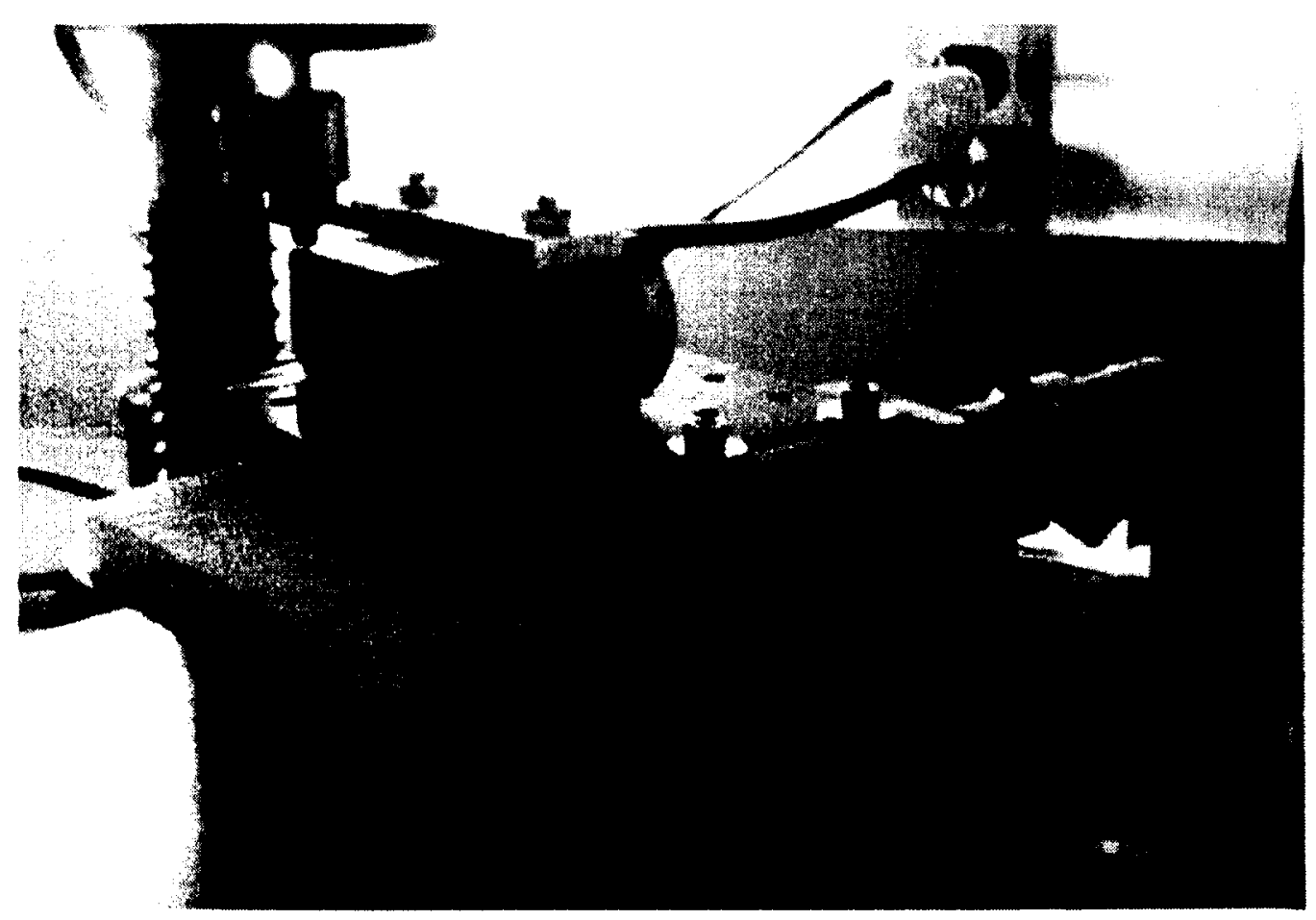

Figure (A.21): Drilling $1 \mathrm{~mm}$ diameter holes in glass tubes

heating the tube carbon heater beyond its maximum operating temperature of $90^{\circ} \mathrm{C}$. Fixing the thermocouples before applying the heater was impractical due to the interference thermocouples imposed on the heater manufacturing process. Bonding with different thermally and electrically conductive adhesives, it was found that a greater conduction rate was often associated with lower mechanical strength and chemical resistance as the high metal content in the epoxy would decrease the bond strength. This was evident when the thermal cycling of a temperature sensor fixed with Omegabond 200 up to $150^{\circ} \mathrm{C}$ was found to readily deteriorate the epoxy as noted in [69]. The resin in Omegabond 200 is reported to contain over 50 percent aluminium oxide [70], while the resin in J-B weld reported to contain 10-20 percent iron powder [71]. The use of high thermal conductivity epoxy was not critical as the value of interest in this study was the temperature difference between thermocouples, thus the radial temperature difference across the epoxy would be nearly equal between the top and bottom thermocouples and result in cancellation. 


\section{A.5.3 Thin tube thermocouples}

Placing the thermocouples on a thin tube was less labour intensive than with a thick tube since no drilling was performed. The main challenge with this task involved dealing with the minute and delicate nature of the tube and joining it to thermocouples of comparable size. Firstly, a different size and sheathing was used for the thermocouples compared to the thick tubes. Teflon coated T-type $0.076 \mathrm{~mm}$ diameter thermocouples (Omega TC-TT-T-4036) were used. Smaller thermocouples were needed compared to the thick tube because the large ones would act as heat sinks and their size would impose considerable stress on the tubes.

The adhesion process for the thin tubes involved taping the heater leads of the tubes to a piece of sheet metal for fixation. A thermocouple was then lightly dipped into epoxy (J-B Weld) such that a bead covered the size of the thermocouple head. The thermocouple was then fixed to the tube and held in place by taping the thermocouple leads to the same piece of sheet metal as the heater leads. A ruler was used to gage the spacing for affixing the second thermocouple in the same manner as the first to achieve consistency between tubes with this freehand approach. High precision of thermocouple placement was not critical since the actual thermocouple spacing was later measured using the microscope reticle, linear stage, and deflectometer to get an accurate distance measurement within $\pm 0.05 \mathrm{~mm}$ (thin) and $\pm 0.08 \mathrm{~mm}$ (thick) to be used in calculating the temperature gradient.

\section{A.5.4 Heating methods}

Applying heat to create a temperature gradient across the meniscus was performed by placing a resistive element on the tube and using a variable power supply as a source to increase the heat load which in turn increased the axial temperature gradient along the wall. This was not a trivial task as several techniques were examined thoroughly before finding a 
method which worked for the testing performed.

\section{Nichrome wire}

The first heating method involved using a 38 AWG nichrome $(\mathrm{NiCr})$ wire having a diameter of $0.10 \mathrm{~mm} \pm 0.005 \mathrm{~mm}(0.004$ inch $\pm 0.0002 \mathrm{inch})$ with a resistance per length of 0.133 $\Omega / \mathrm{mm}$. The wire was manually wrapped helically around the tube top and held in place by twisting the nichrome wire into a multistranded lead and soldering the pair together. Two methods by which to apply and maintain tension were attempted. The first method was to simply nylon ratchet tie wrap the tube at one end of the heater, then wrap the helix manually while maintaining tension, and finally tie wrap the lead at the other end of the heater. This method did not fix the wire to the tube tightly enough allowing for slip which resulted in tension loss and a loose contact between the wire and tube. The second method to maintain wire tension was to wrap it around the tube with one end fixed and using a clamp having a leadscrew which when turned would impart tension on the wire. The tension was such that the wire was taut enough to wrap around the tube without slipping and provided firm contact with the glass without snapping the wire or tube. This arrangement is shown in Figure (A.22). Issues were found with the nichrome wire heating method in terms of how to consistently manually wrap a helix around such a small cylinder. This lack of reproducibility would make comparison between tests less valid. Wire snapping was also prevalent as the amount of tension to provide was subject to a lot of guesswork and having a good sense of craftsmanship. During testing in ambient air, it was found that as the wire was heated and the meniscus evaporated, the rising vapour would condense on the wall between the helix windings and descend back onto the meniscus, disturbing the evaporation process as an artifact of the heating method. To solve this would require winding the helix with a much smaller pitch, but this was deemed overly complicated and practically impossible. Finally, the tension required to make firm contact with the heater would not be sustainable 


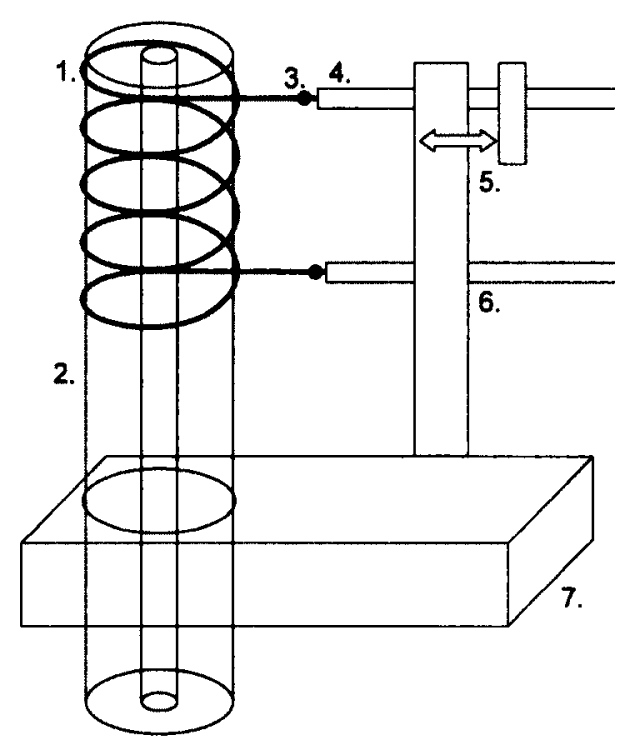

Figure (A.22): Helix heater configuration: 1. nichrome heating element, 2. thick walled capillary tube, 3. soldered junction, 4. insulated wire, 5. tensioner clamp, 6. fixed clamp, 7. base to carry tensioning load

with a thin tube being much less robust than its thick counterpart.

\section{Film heater on cylindrical copper annulus}

The second heating arrangement shown in Figure (A.23) involved wrapping a Kapton film heater (Omega KHLV-0502) containing an etched element with a power density of 1.55 $W / \mathrm{cm}^{2}$ distributed over a $1 \mathrm{~cm}$ by $5 \mathrm{~cm}$ rectangle onto a copper annulus machined on a lathe with an outer diameter and thickness such that the entire surface was covered by the film heater without overlap. The inner diameter was sized for tube clearance. Epoxy (JB Weld) was used to mount the heater to the outer wall of the annulus and thermal paste was used between the inner wall and glass tube. This heating method provided a more even heat distribution compared to the helical nichrome wire heating method. This method was not used because the weight of the copper saddle was significant enough to require external tube support other than the leads. Also, the greater thermal expansion of the copper saddle compared to the glass tube would create clearance between the tube and the saddle. 


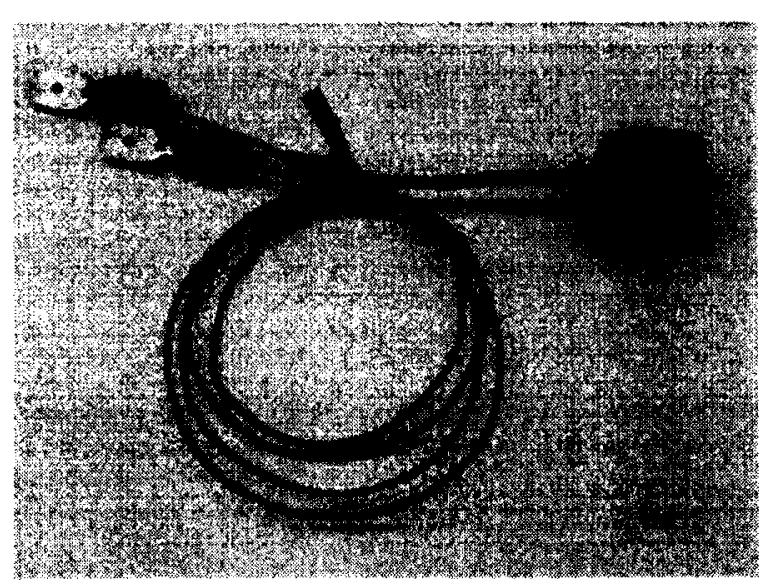

Figure (A.23): Heater setup using film wrapped around copper annulus

Finally, making heaters for tubes with cross sections other than circular would require more elaborate machining of a copper saddle using a mill, broach, or electric discharge machining to make the cavity for tube residency. This method was also abandoned.

\section{Carbon film deposition heater}

The chosen method for heating both the thin and thick tubes was the deposition of carbon conductive composition within a polymer thick film (Dupont 7102). The manufacturing procedure is described below. The benefits of this method were uniformity of heat application, light weight, good chemical and mechanical resistance, and repeatability.

The heater deposition step was performed before mounting of thermocouples on both the thick and thin tubes since they would hinder the process, and after the thermocouple cavity holes were drilled for the thick tube.

To begin, the tube was wrapped in masking tape to prevent overspray from landing inside the tube or on the outside wall needing transparency for observation. The area for deposition was cleaned with an acetone dipped wipe to ensure adhesion to a clean surface. Afterwards, the tube was mounted into a small lathe consisting of a 3/8 inch keyed chuck mated concentrically to a geared down DC motor shaft shown in Figure (A.24). A 


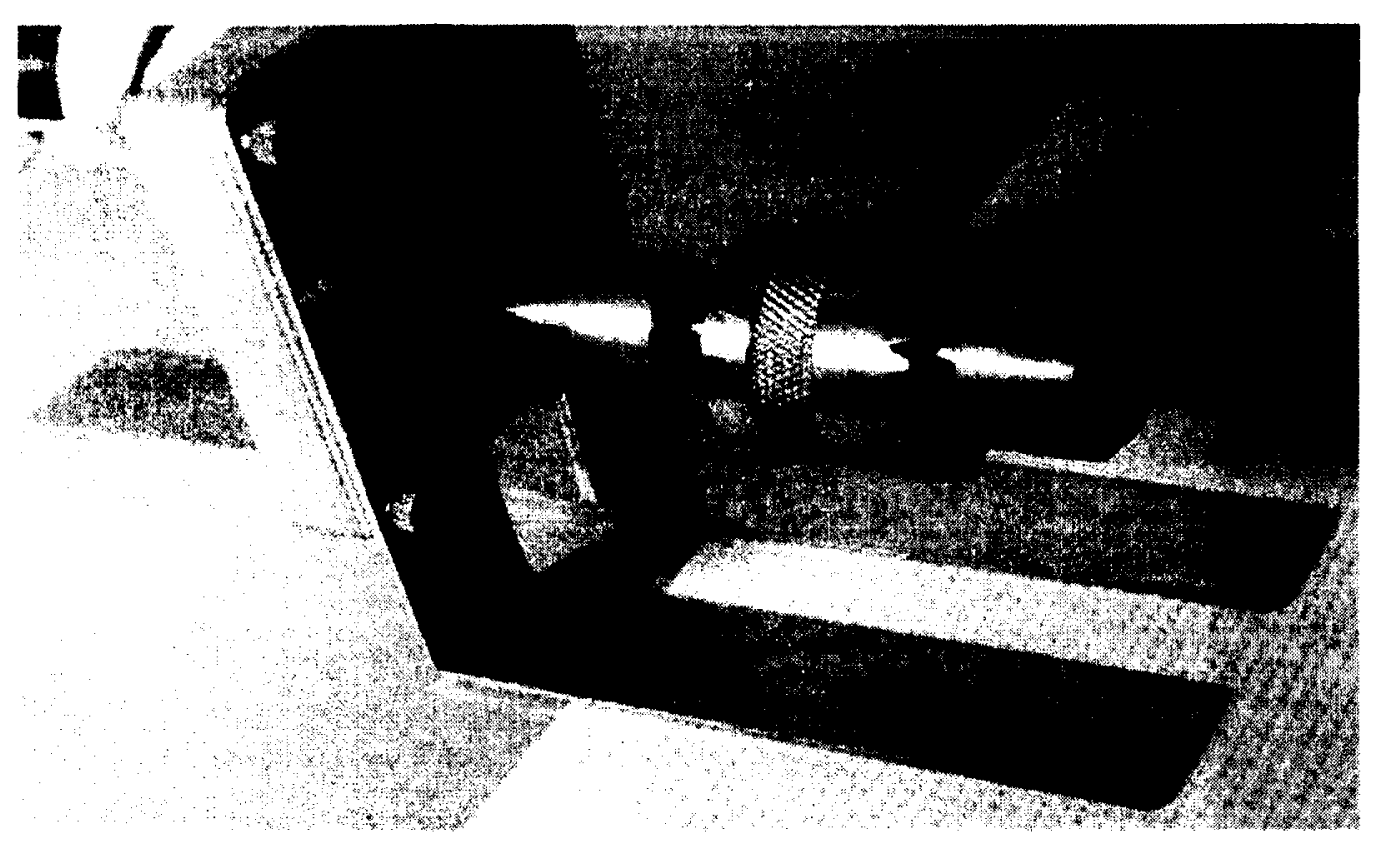

Figure (A.24): Rotary drive for heater deposition

mark on the chuck was used as a reference to indicate a full revolution of the tube during which spraying would be performed and promptly cease at the end to avoid over spray and subsequent dripping.

The spraying apparatus consisted of a gravity fed double action, $0.35 \mathrm{~mm}$ needle diameter airbrush (Iwata Eclipse HP-CS) connected to a high pressure ultra high purity nitrogen cylinder with a two stage regulator reducing the feed pressure to 2 bar. The carbon composition compound having a high viscosity required mixing in a $1: 1$ by volume ratio with the solvent diethylene glycol monomethyl ether (DGME) so that it would become a colloidal suspension upon exiting the airbrush nozzle.

After each revolution during which the tube was sprayed, hot air was applied via a hand held heat gun while the tube was rotated to accelerate the drying process by normalising convection. Hot air was applied until the film changed colour from a glossy to matte black, indicating the layer was dry since the solvent had mostly evaporated. The rotary drive was then stopped and the resistance across the heater was measured by gently applying 
the leads of an ohmmeter to both ends of the coating. As resistance would decrease with the number of layers, the process was repeated usually between 10-20 times so that the resistance would fall from the $1000 \Omega$ range down to approximately $100 \Omega$.

Once the resistance was reduced to the desired range, a thin band of two part silver epoxy (EPO-TEK H20E) was applied to the top and bottom of the heater. Two wire leads were fashioned with ends contoured to fit snuggly around the perimeter of the corresponding tube. The shape of the wire lead was formed by bending it around a metal cylinder the size of the glass tube since bending it around the tube could damage the glass. Enough clearance was given so that leads would not abrade the silver epoxy strip around the tube while still having a gap small enough that could be bridged by a second layer of silver epoxy. Wire leads were then glued to the bands with the same epoxy. The leads were kept stationary with respect to the tube by taping them both to a sheet metal base which was placed on a hot plate at $150^{\circ} \mathrm{C}$ to cure for 10 minutes. After curing, the heater and leads were covered with a mechanically stronger epoxy (J-B Weld) having negligible electrical conductivity and good chemical resistance to solvents used in cleaning and testing for this study. A final resistance test would typically show a drop to $30-35 \Omega$ across the leads. This is due to the silver epoxy bands which lower the overall resistance by providing an evenly distributed and relatively highly conductive pathway for current to flow from one heater end to the other.

\section{A.5.5 Progression of tube manufacturing}

Figure (A.25) shows the steps starting with a stock tube which is cut to length after which holes are made and then the carbon heater layer is applied. Afterwards, the heater leads were epoxied to increase electrical conduction and then epoxied again for chemical resistance. Finally, the thermocouples were epoxied and the leads are clamped. Comparatively, 


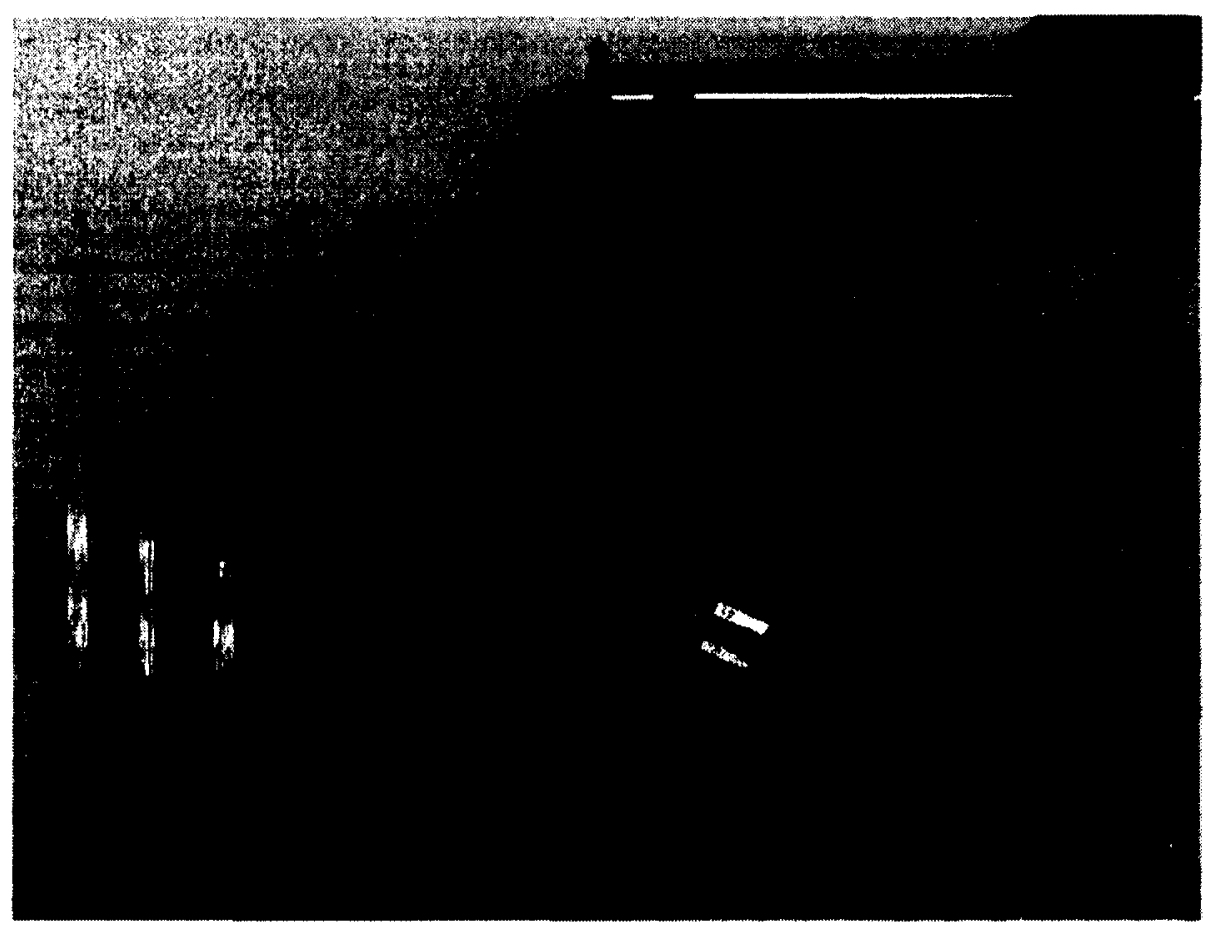

Figure (A.25): Evolution of capillary tube from stock to working: 1. offcut stock, 2. length cut to size, 3a. heater silver contact band, 3b. bare carbon composition, 3c. TC holes $3 \mathrm{~d}$. taped off opening, 4a. epoxy layer, $4 \mathrm{~b}$. heater lead, 4c. TCs, 4d. lead bracket clamp, 4e. support member, $4 \mathrm{f}$. crimped leads, $4 \mathrm{~g}$. TC subminiature connectors

A finished thin capillary tube is shown in Figure (A.26) to give a sense of the size and fragility of thin capillary tubes.

\section{A.5.6 Lead bracket clamp}

Suspending the tube and submerging it into the reservoir required holding it in some manner. The stiffness of the heater leads was enough to bear the tube weight without it moving. When initially manipulating the thin tubes, the small thermocouple junction would frequently break without support. Placing the thermocouple leads in parallel with the much stiffer heater leads eliminated this problem via the clamp shown in Figure (A.25). A sandwiching clamp consisting of two outer aluminium flats with a piece of flexible gasket material was held together using two bolts with shaft clearance holes for one of the aluminium 


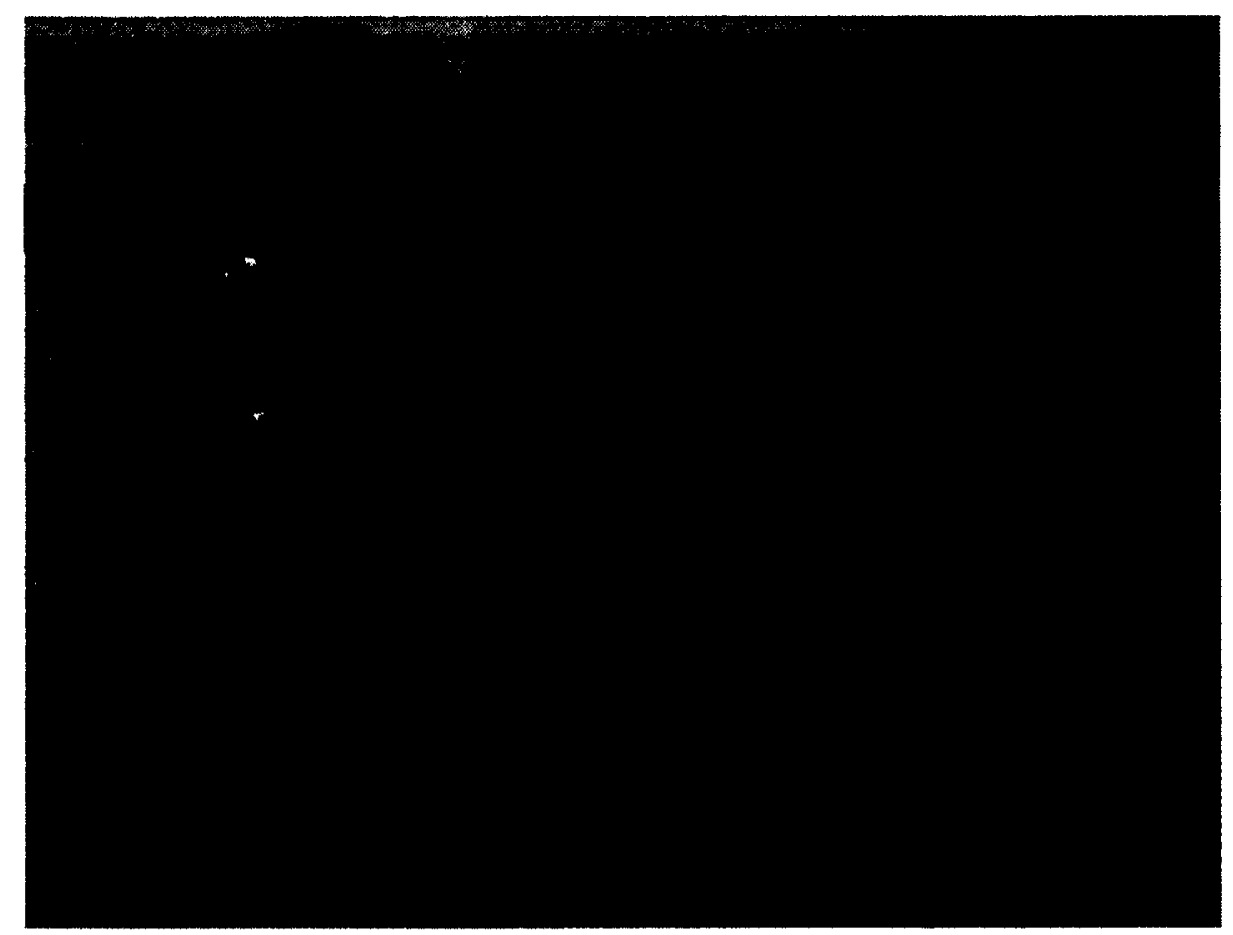

Figure (A.26): Thin tube (0.5 mm ID) with thermocouples and lead bracket clamp

flats and gasket and threading into the remaining flat.

Special care was taken when holding the thermocouples connected to the thin tube as minimal force was required to shear them apart. By clamping the thermocouple leads in parallel with the heater leads, they were resilient enough to withstand the manipulation required for testing. The tube could also be moved with respect to the clamp for fine tune adjustments by bending the heater lead wires. Extra slack was provided to the thermocouple leads to that this bending action would not over tension the leads.

\section{A.6 Tube cleaning}

Maintaining a clean surface outside and particularity inside the capillary tube was important as it would affect interfacial interaction. Particles inside the tube could act as hinge points if on the wall or disrupt the convection currents if moving within the tube. The delicacy of the 
thin tube and the fact that the attached thermocouples and heater were not resistant to harsh but more thorough chemical cleaning lead to the following cleaning method. An ultrasonic cleaner (Branson 2510) emitting a frequency of $40 \mathrm{kHz}$ in a tank (241 x $140 \times 102) \mathrm{mm}^{3}$ was used to clean the internal tube test section. A double bath was formed with water contacting the ultrasonic cleaner reservoir and acetone contacting the tube. Degassing of the baths using the cleaner functionality was performed before cleaning the tubes.

By taking advantage of the capillary rise due to surface tension, it was possible to keep the thermocouples and heater on the exterior of the tube dry while still cleaning the internal diameter. A typical cleaning session is shown in Figure (A.27) where the tube bracket and horizontal beam arrangement were used to suspend the tube in an acetone reservoir residing in a water bath acting as the source ultrasound medium. The tube was arranged such that the meniscus formed by the acetone reached near the top of the heater and could be seen by looking down from the tube exit. By placing the tube at an angle with respect to gravity, the capillary rise was adjustable such that it could cover the entire inner diameter of the tube without the submersion of the heater element. The bath was monitored to avoid excessive temperature rise due to the energy input from the cleaner. After the acetone bath, the tube was primed with $\mathrm{n}$-pentane to wash away any residual acetone. The effect of cleaning was observable when comparing the capillary for a cleaned tube versus that for one purposely left unclean. It was found that the capillary would not move in a linear relation with the reservoir adjustment even without heat input. The priming of the tube as it makes initial contact with the reservoir was also not complete with the unclean case as the uneven wall would trap vapour which would then rise out of the tube due to buoyancy. 


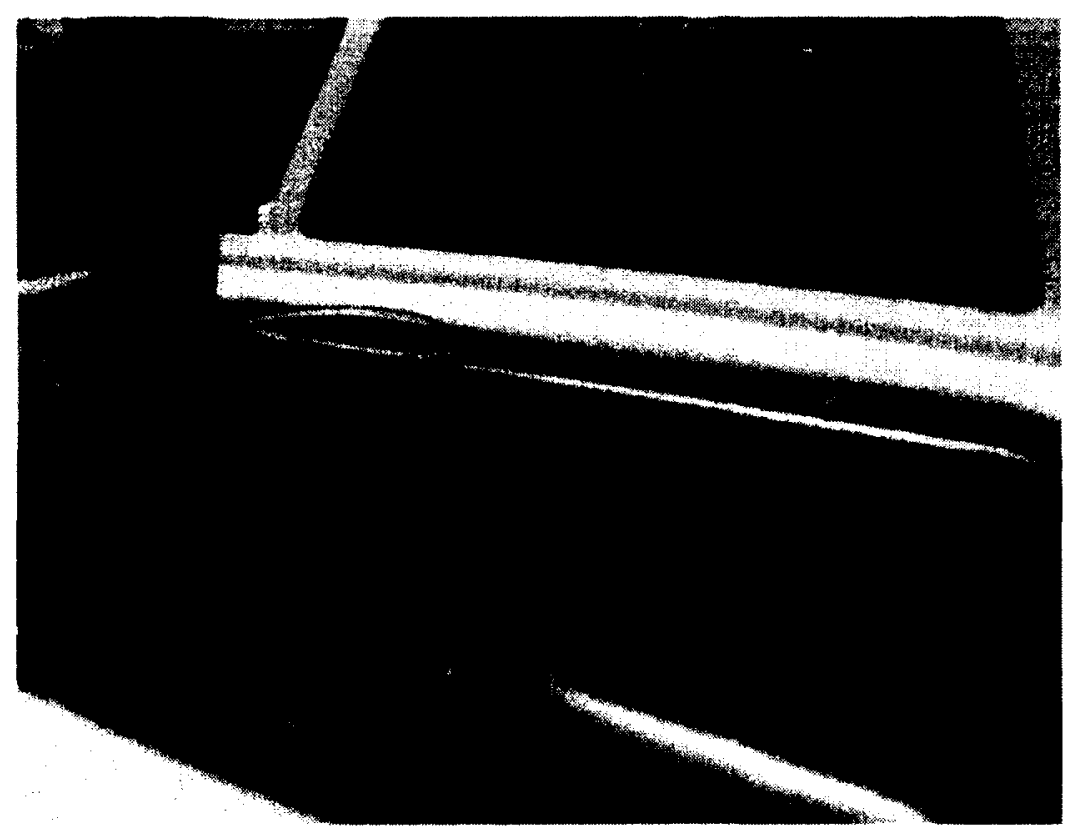

Figure (A.27): Tube being washed in the ultrasonic cleaner double bath of acetone in water

\section{A.7 Heater insulation}

Insulation of the tube heater was considered as a means to reduce the heat required to destabilise the meniscus and reduce the heat exchange with the ambient. The insulation would have to withstand various conditions such as being placed in a vacuum, exposed to a solvent, and being heated until the meniscus destabilised.

The first insulation method attempted involved wrapping the tube with Teflon tape considering its chemical inertness and thermal conductivity $k=0.25 \mathrm{~W}(\mathrm{mK})^{-1}$. Testing undergone using Teflon wrapping showed too low of a temperature gradient radially across the insulation through thermocouple measurement to warrant further use.

The chosen insulation method was a spiral of Aerogel (Pyrogel 2250) held in tension with the weaving of Teflon coated fibreglass thread. The Teflon coating prevented the thread from fraying. The outer surface of the aerogel was wrapped with Teflon tape for 
shape maintenance and due to the fibre shedding tendency of the Aerogel when manipulated. This was important to avoid for contamination and maintaining clarity for microscopy. A piece Aerogel was tested for chemical compatibility with n-pentane and was found to be intact after days of being submerged in a n-pentane bath. The thermal conductivity of the Aerogel was an order of magnitude lower than Teflon with $k=0.015-0.017$ $\mathrm{W}(\mathrm{mK})^{-1}[72]$. Testing showed a large radial temperature gradient across the insulation as a testament to its effectiveness.

In retrospect, the heater insulation provided little benefit as testing without insulation was still feasible and the heat required was small enough $(\lesssim 1 W)$ such that the chamber was able to dissipate the heat. Insulation may be more useful in future testing for determining the heat applied to the meniscus given that the convective losses are difficult to accurately predict, thus their reduction would lead to an overall lower error.

In order to determine whether insulation is helpful or detrimental in decreasing heat loss, we calculate $r_{c}=k / h$, the critical radius of insulation for a cylinder assuming one dimensional radial heat flow [73]. Using the known aerogel insulation thermal conductivity leaves only the determination of the convection heat transfer coefficient, $h$ in order to calculate $r_{c}$. We first check if the inequality in Equation (A.3) is satisfied to justify the use a flat plate approximation can be used for natural convection from an isothermal surface [74]

$$
\frac{d}{L} \geq \frac{35}{G r_{L}^{L / 4}}
$$

where $G r_{L}$ is the Grashof number which represents the ratio of buoyant to viscous forces expressed as

$$
G r_{L}=\frac{g \beta \Delta T L^{3}}{\nu^{2}}
$$

$\beta=\frac{1}{V}(\partial V / \partial T)_{P}$ is the volumetric thermal expansion coefficient and it can be shown that $\beta=T^{-1}$ for ideal gases. The characteristic length, $L$ is the height of the wall. Assuming a 
tube diameter of $1.2 \mathrm{~mm}$, heater height of $10 \mathrm{~mm}$, and wall to vapour temperature difference of $\Delta T=350-300 \mathrm{~K}$, the inequality in Equation (A.3) is not satisfied. We thus use the relation in Equation (A.5) which accounts for the tube curvature to obtain the Nusselt number, $\overline{N u}_{L}=\bar{h} L / k_{v}$ with properties evaluated at the film temperature $T_{f}[75]$

$$
\overline{N u}_{L}=\frac{4}{3}\left[\frac{7 R a_{L} P r}{5(20+21 P r)}\right]^{1 / 4}+\frac{4(272+315 P r) L}{35(64+63 \operatorname{Pr}) d}
$$

where $d$ is the outer tube diameter and $R a$ is the Rayleigh number which is the product of the Grashof and Prandtl numbers. The resulting convective heat transfer coefficient, $h_{c}=31.5 \mathrm{Wm}^{-2} \mathrm{~K}^{-1}$ corresponds to a critical radius, $r_{c}=0.56 \mathrm{~mm}$. Since $2 r_{c}<d$, insulating circumferentially with any amount of aerogel would decrease heat loss.

\section{A.8 Reservoir cooling}

To demonstrate the predictive ability of the stability theory later developed, changing the reservoir temperature would provide an additional test parameter for comparison. Since heating the reservoir would result in excessive evaporation and potentially boiling, cooling was attempted. The method used for cooling of the reservoir proved unsuccessful, but the methodology used is described below to provide a first iteration for further work.

\section{A.8.1 Possible cooling methods}

A challenge pertaining to cooling the internal reservoir is the requirement to keep the cooling system closed from the test environment in order to maintain fluid purity while also having the cooling system flexible enough for the reservoir to remain adjustable with respect to the capillary tube. 


\section{A.8.2 External cooling}

By cooling the test liquid outside of the chamber, the need for an internal cooler is eliminated provided that testing can be performed before the reservoir temperature reaches room temperature. A slurry of dry ice in acetone could be used to cool the test liquid in a double bath.

Alternatively, cooling the shell of the vacuum chamber via a heat exchanger or dry ice could maintain the chamber well below ambient room temperature. A problem that arises with this approach is that global cooling would facilitate excessive condensation at the walls and soaking of the chamber walls and floor when compared to local cooling. As an alternative to global external cooling, local external cooling of the reservoir could be achieved by attaching a braided copper strap between the reservoir base and chamber floor. The extent of the strapping would be limited by the increased stiffness that additional strapping would impart on the connection since it would act as springs in parallel. A cold liquid such as acetone cooled by dry ice could be used to remove heat from the chamber floor.

Another approach could be external heat extraction via heat pipes. The intrinsic passivity of this approach would result in low vibrational input to the chamber which could affect the meniscus. Absence of maintenance would also be beneficial for reducing experimental complexity.

\section{A.8.3 Internal liquid heat exchanger}

Running a cooled liquid line to and from the chamber would provide adequate cooling to maintain the reservoir below ambient. A challenge with this approach is line flexibility to allow for reservoir positioning. Nylon lines could provide enough flexibility while still maintaining sufficient reservoir position control. An external chiller could be used to run a 
line with water and ethylene glycol with the flow rate controlled using a bypass valve. The flow rate would be kept to a minimum that would cool the reservoir to minimise vibration caused by the liquid flow. To transfer heat from the reservoir to the coolant, a copper jacket with baffles to aid in mixing and reduce vibrations by reducing speed could be placed around the reservoir in contact with the reservoir wall and floor. The assembly could be brazed with significant lapping to insure a vacuum seal and provide rigidity for the structure to withstand internal atmospheric pressure during chamber evacuation.

\section{A.8.4 Thermoelectric cooling}

By placing a thermoelectric cooler (TEC) inside the vacuum chamber, local cooling could be achieved at the reservoir. Net heat is generated by the Peltier element and must be directed away from the reservoir in order to maintain its temperature colder than ambient. In thinking the heat transferred to the reservoir and that generated by the TEC could be convected to the chamber walls and out to the test room, cooling the reservoir was attempted thermoelectrically. We also thought there could have been sufficient time to perform the experiment before the heat output by the TEC would be too great to locally cool the reservoir. The TEC setup which was placed in the vacuum chamber in an attempt to cool the reservoir is shown in Figure (A.28). 


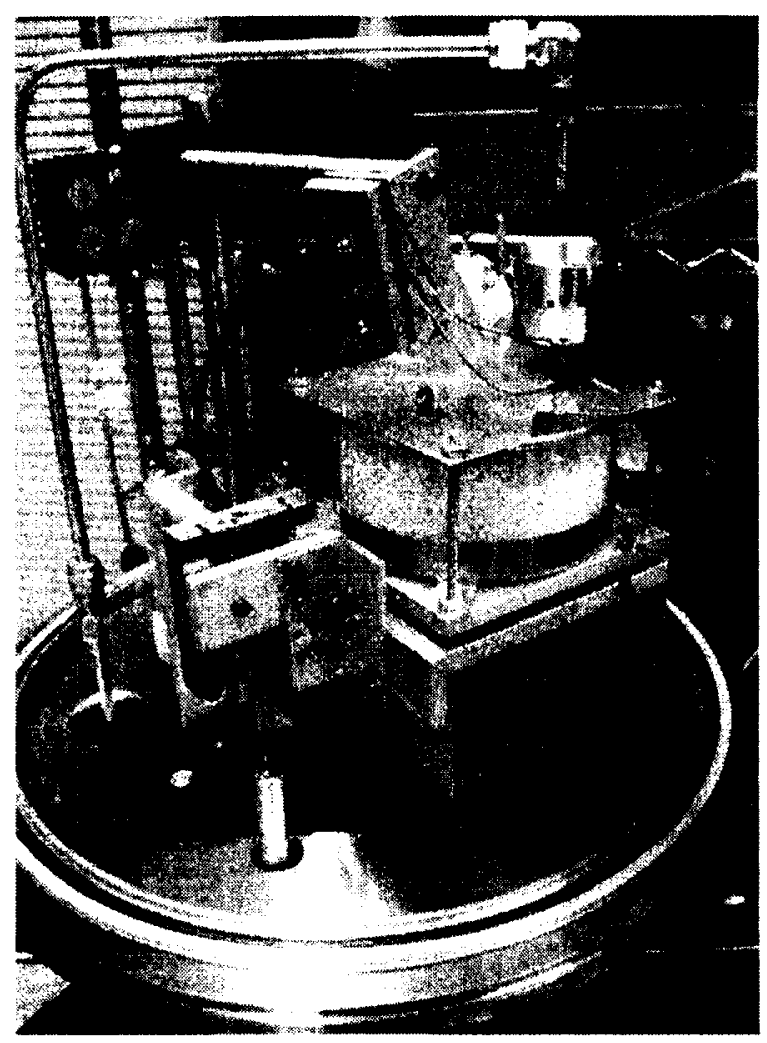

Figure (A.28): TEC setup for temperature control: 1. funnel, 2. reservoir cover, 3. cold plate (above TEC), 4. heat sink (below TEC)

The TEC (Custom Thermoelectric 12711-9L31-06CW) was square with a width of 50 $\mathrm{mm}$ and a maximum cooling capacity of $51 \mathrm{~W}$. To accommodate for the TEC, a square aluminium bonded fin heat sink $102 \mathrm{~mm}$ wide was connected to the linear stage arm. The TEC was sandwiched between the centre of the heat sink and a square aluminium plate 6.35 $\mathrm{mm}(0.25 \mathrm{inch})$ thick with Arctic Silver 5 thermal grease. Clamping force was maintained using two 6-32 stainless steel screws thermally isolated from the heat sink using nylon shoulder washers and threaded into the cold plate. Belleville washers were placed between the screw heads and nylon shoulder washers to allow for thermal expansion of the TEC without excessive stress. The reservoir was placed on the cold plate over a layer of the same thermal grease. A blind hole was drilled into a side of the cold plate for sensing its temperature and using the information to regulate power to the TEC using a temperature 


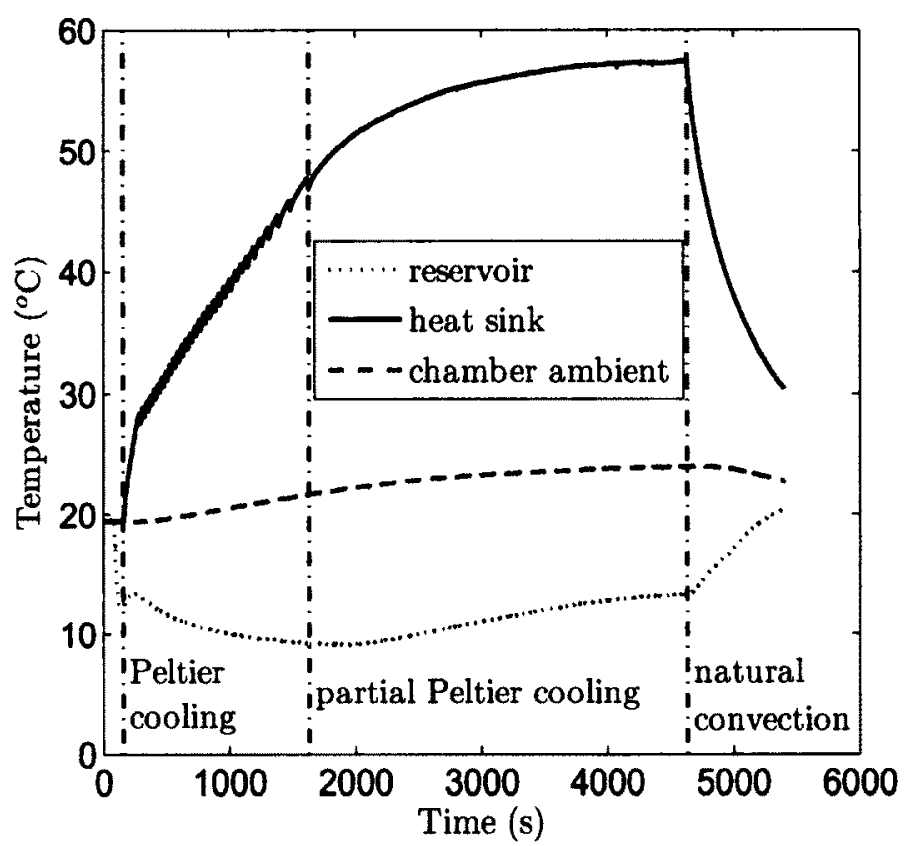

Figure (A.29): Ambient test showing partial effectiveness of TEC with $20 \mathrm{~W}$ input on TEC controller (Omega CNi3244) in deadband cooling mode.

To decrease the rate of convective heat transfer at the surface of the reservoir, a stainless steel cover was affixed. A notch was made at the perimeter near the microscope view port for submerging the capillary tube. A stainless steel funnel was fusion tack welded and then epoxied to the plate in order to reduce size of the fill hole while still capturing the majority of the liquid pumped into the chamber.

Before testing in vacuum conditions, a preliminary ambient test was performed to judge how well the TEC would perform without being contained by the chamber shell, thus being able to transfer the heat it produced more efficiently than when sealed. The results of this test are shown in Figure (A.29) with the TEC drawing $20 \mathrm{~W}$ of power. As power to the TEC was initially applied, the temperature of the reservoir dropped and the TEC was able to maintain a cold plate temperature between $5-6^{\circ} \mathrm{C}$ using the thermal controller. The discontinuous nature of the sink temperature profile is a result of the controller switching 
the TEC on and off. Since the TEC was able to cool the reservoir in addition to extracting the heat added by convective heating of the reservoir, there could exist a lower input power setting that would simply maintain the reservoir temperature and thus produce less heat to transfer away. This power setting was not sought, but could be determined experimentally to minimise heat input. To the right of the region mentioned above, the TEC is no longer able to lower the reservoir temperature, resulting in the reservoir temperature increasing up to a quasi-steady state where the reservoir and heat sink temperatures near constancy. The power is then shut off and natural convection heats up the reservoir. Comparing the temperature change in time before and after the TEC was shut off, we see the rate in reservoir temperature increase was lower than with pure natural convection.

When testing with the chamber closed and saturated with n-pentane, we notice the TEC was not able to extract heat to the outside of the chamber faster than its production and absorbtion by the reservoir, leading a monotonically increasing reservoir temperature as shown in Figure (A.30).

Insulation of the reservoir wall with a layer of Aerogel to decrease convection losses was attempted. In order to prevent liquid n-pentane from wetting the insulation and degrading its effectiveness, a polyvinyl chloride (PVC) film was applied to the insulation as a barrier. Since initial chamber evacuation would not release gas sealed by the film, the internal space enclosed by the film was evacuated. Unfortunately, vacuum sealing of the Aerogel insulation was unsuccessful as the vacuum pulled on the insulation was significantly weaker than that applied to the chamber, resulting in the expansion of the PVC jacket and the breaking of its seal when submitted to the chamber evacuation procedure. 


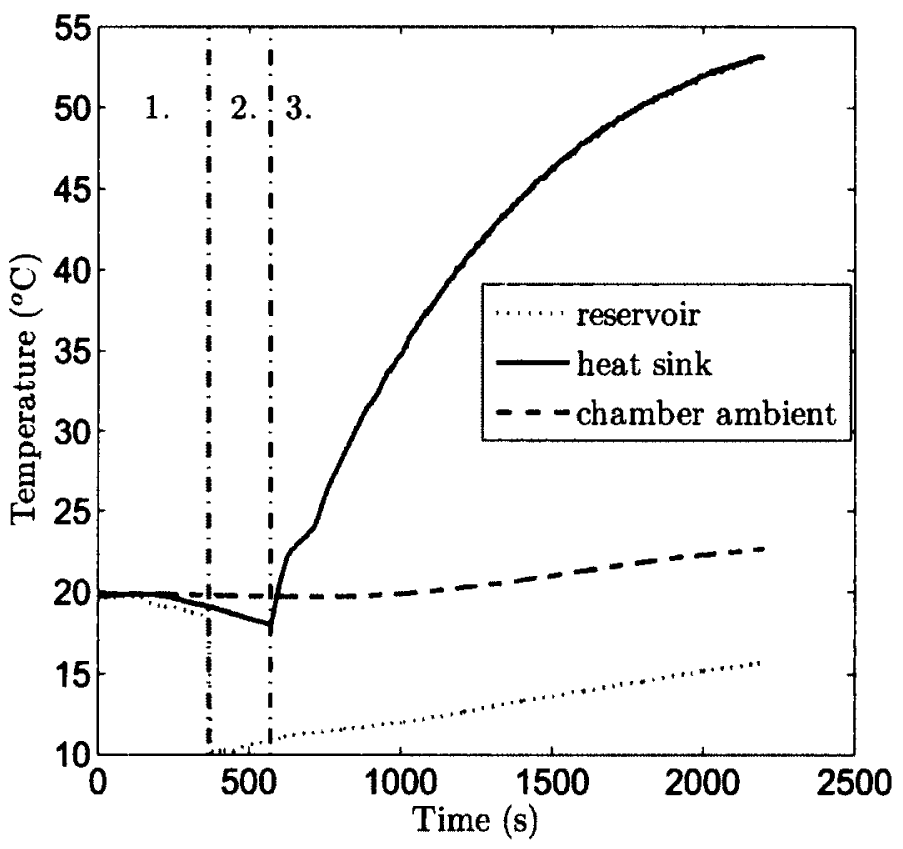

Figure (A.30): Attempting to cool chamber reservoir: 1. evaporative cooling from $n-$ pentane entering vacuum chamber, 2 . liquid n-pentane contacts reservoir thermocouple, 3. TEC on and unable to cool reservoir

\section{A.8.5 Future testing}

In future work, if one were to use the TEC to cool, a more efficient thermally conductive pathway to extract the heat from the chamber should be developed. Given the requirement for linear motion of the reservoir, the flexible braided copper meshing or a heat pipe as mentioned above could be used to join the hot side of the TEC and chamber floor. To further extract heat from the floor, a dry ice and acetone slurry could be placed in a nook situated outside the chamber under the contact area covered by the braided copper meshing. If one were to abandon the TEC altogether which is the recommendation of the author, liquid cooling as suggested above should be used as it appears to be a more feasible method since it does not add heat to the system which would need to be transferred away from the reservoir. The TEC solution initially seemed like a quick fix that could work without introducing a liquid cooling system which may be more complex to implement. Being 
aware of the net heat produced by the TEC, we thought that the transient period when local cooling is feasible would be longer, but this proved incorrect after testing and realising the time scale of TEC warming period was smaller than that of a typical test. 